\title{
WestVirginiaUniversity
}

THE RESEARCH REPOSITORY @ WVU

Graduate Theses, Dissertations, and Problem Reports

2007

\section{Basic building blocks of real-time data analysis as applied to smart oil fields}

Daniel G. Gonzalez

West Virginia University

Follow this and additional works at: https://researchrepository.wvu.edu/etd

\section{Recommended Citation}

Gonzalez, Daniel G., "Basic building blocks of real-time data analysis as applied to smart oil fields" (2007). Graduate Theses, Dissertations, and Problem Reports. 1825.

https://researchrepository.wvu.edu/etd/1825

This Thesis is protected by copyright and/or related rights. It has been brought to you by the The Research Repository @ WVU with permission from the rights-holder(s). You are free to use this Thesis in any way that is permitted by the copyright and related rights legislation that applies to your use. For other uses you must obtain permission from the rights-holder(s) directly, unless additional rights are indicated by a Creative Commons license in the record and/ or on the work itself. This Thesis has been accepted for inclusion in WVU Graduate Theses, Dissertations, and Problem Reports collection by an authorized administrator of The Research Repository @ WVU. For more information, please contact researchrepository@mail.wvu.edu. 


\title{
BASIC BUILDING BLOCKS OF REAL-TIME DATA ANALYSIS AS APPLIED TO SMART OIL FIELDS

\author{
Daniel G. Gonzalez
}

Thesis submitted to the

College of Engineering and Mineral Resources

at West Virginia University

in partial fulfillment of the requirements

for the degree of

\author{
MASTER OF SCIENCE \\ IN \\ PETROLEUM AND NATURAL GAS ENGINEERING
}

\author{
Shahab Mohaghegh, Ph.D., Chair \\ Razi Gaskari, Ph.D. \\ Sam Ameri, M.S
}

Morgantown, West Virginia

2007

Keywords: Smart Field, Real Time Data, Reservoir Management. 


\title{
Abstract \\ BASIC BUILDING BLOCKS OF REAL-TIME DATA ANALYSIS AS APPLIED TO SMART OIL FIELDS
}

\author{
Daniel Gonzalez
}

The process of continuously monitoring and analyzing data in real time as well as reacting to events in an effective and more efficient manner represents one of the challenges in the oil and gas industry today. The existence of a computer model that is capable of evaluating data that enters a computer database in real time, performs different kinds of production analysis, and predicts reservoir performance opens new possibilities for field optimization. Having a model that alerts the engineers of abnormal situations during production operations will help to decrease the time of reaction to such events and as a result will optimize the processes.

The first building blocks for Smart Field Data Analysis that will serve as the bridge for building a more complex computer model to evaluate the data as it is being measured from the production fields; forecast production behavior and report unexpected situations from the measurements has been be the goal of this study.

A computer program (Smart Field Data Analysis-SFDA 1.0) capable of reading high frequency data and presenting it in real time has been created. One of the main problems encountered during this process was dealing with high frequency data. A process that summarizes this type of data while preserving its integrity has been developed. After the high frequency data has been summarized different types of production analysis have been performed using actual real time data generated from a storage field provided by Columbia Gas Transmission Corporation. Material balance analysis has been carried out in order to estimate gas in place and determine whether a leak is presented in the reservoir. Decline Curve Analysis has also been included to forecast gas production. In addition, the program is capable of detecting pressure transient periods in which the high frequency data containing such information will be stored and accessible for well testing analysis. Finally, real time data using Eclipse Simulator has been generated and tested during different scenarios to validate the work previously done. 
To my mother, Beatriz; my father, Gilberto; my sister, Cristina; and my brother Manuel. 


\section{Acknowledgements}

I would like to thank and express my immense gratitude to my research advisor, Dr. Razi Gaskari, for his continuous support and assistance throughout my research. His constant guidance and supervision has been very valuable during this study. Besides being an excellent advisor, he has also been a friend to me. Moreover the knowledge Dr. Razi Gaskari has imparted to me will be very helpful during my professional career.

I would also like to extend my appreciation to Dr. Shahab Mohaghegh for his endless supervision and cooperation to develop this work. His continuous technical advice has been very useful to me during this educational process.

I would also like to share that it has been a privilege for me to have come to this great university and to have been a part of the Petroleum Engineering program. I would like to thank the entire faculty members and the chairman Prof. Sam Ameri, for their constant support and encouragement during my studies at West Virginia University.

I am very thankful for all the friends I have met during the past two years with whom I have plenty of memories. I would like to thank my office mates who have been very helpful during good and difficult situations and with whom I have great anecdotes especially: Domingo Mata, Delal Gunaydin, Jalal Jalali, Miguel Tovar, Cesar Silva, and Kazim Malik.

Finally, I would like to thank my father and mother who have been very supportive during this stage of my life and who have been an inspiration for me. 


\section{Contents}

Abstract $\quad$ ii

Acknowledgments $\quad$ iv

1 Introduction and Motivation 1

1.1 Statement of the Problem............................................

1.2 Schematic of the Program............................................. 3

2 Theoretical Background 4

2.1 Reservoir Management...............................................4

2.1.1 Smart Fields..................................................4

2.1.2 Real Time Data...........................................4

2.1.3 High Frequency Data........................................5

2.1.4 Real Time Data Acquisition...................................5

2.1.4.1 Data Cleansing....................................5

2.1.4.2 Data Aggregation..................................6

2.1.4.3 Data Integration....................................6

2.1.5 Models and Detection.......................................6

2.1.5.1 Detection........................................

2.1.5.2 Decline Curve Analysis..............................

2.1.5.3 Material Balance with Interference.......................

2.1.5.4 Reservoir Simulation............................. 8

2.1.5.5 Data Driven Tools.....................................8

2.2 Long Term Data Analysis............................................ 8

2.2.1 Outlier Removal............................................8

2.2.2 Denoising ...................................................

2.2.3 Transient Identification..................................... 10

2.2.4 Data Reduction..........................................11 
2.3 Permanent Downhole Equipments used in Smart Fields....................12

2.4 Real Time decision making while drilling............................13

2.4.1 Real Time drilling and completion modeling....................13

2.4.2 Abnormal situation detection and management.....................13

2.5 Routine Reservoir Surveillance...................................... 14

2.5.1 Decision making process..................................14

2.5.2 Event Detection...........................................15

3 Methodology 16

3.1 Real Time Measurements.........................................16

3.2 Pressure Transient Analysis and Outlier Removal........................20

3.3 Averaging high frequency data while preserving its integrity................23

3.3.1 Flow Rate average process................................ 30

3.3.2 Pressure average process.................................. 33

3.3.3 Summarized Data Selection Process...............................35

3.4 Pressure, Flow Rate, and Gas in Place Profiles Using Summarized Data......36

3.4.1 Pseudo Pressure Profile........................................36

3.4.2 Pressure square versus time..................................... 37

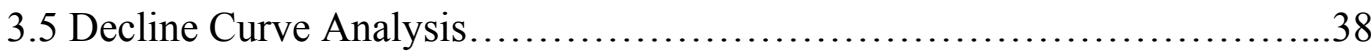

4 Case Study-Results 39

4.1 Real Time Data-Columbia Gas Transmission Corporation...................39

4.2 Pressure Transient Analysis..........................................40

4.3 Summarized Data Results Analysis...................................42

4.4 Material Balance Analysis..........................................53

4.4.1 Gas in Place in Real Time..................................57

4.5 Pseudo Pressure Analyses............................................58

4.6 Pressure Square Analyses........................................60

4.7 Decline Curve Analysis.........................................62 
5 Data Control Environment-Results $\quad 64$

5.1 Real Time Data-Eclipse Simulator................................64

6 Concluding Remarks

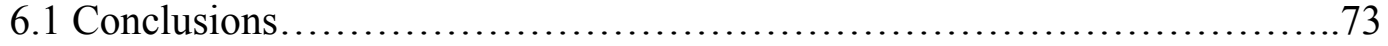

6.2 Future Work ................................................... 74

$\begin{array}{ll}\text { Bibliography } & 75\end{array}$

$\begin{array}{ll}\text { Appendix } & 77\end{array}$

User Manual........................................................ 78 


\section{List of Tables}

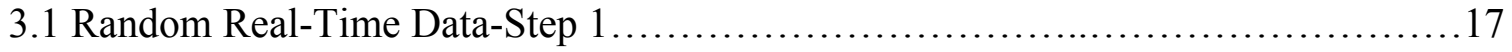

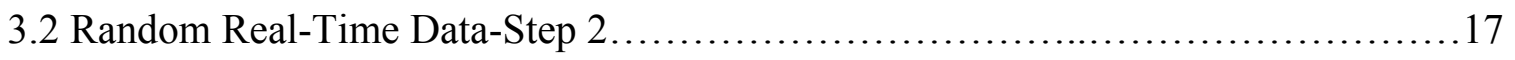

3.3 Random Real-Time Data-Step 3......................................... 17

3.4 Random Real-Time Data-Deleting First Row Process........................... 18

3.5 Random Real-Time Data-Updated Process................................. 18

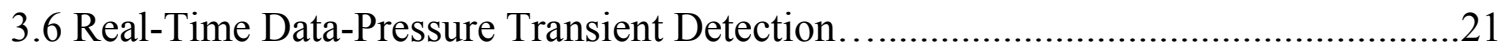

3.7 Real-Time Data-Outlier Detection..........................................

3.8 Random Data-Flow Rate Aver. Process........................................30

$3.9 \Delta Q_{\text {Preset }}$ values at different flow rate values intervals........................... 32

$3.10 \Delta \mathrm{P}_{\text {Preset }}$ values at different Pressure values intervals.............................. 34

3.11 Pseudo Pressures at different Pressure values................................... 37 


\section{List of Figures}

2-1 Schematic of the Program...........................................................

3-1 Real Time Data (Dec 12-2004) ..................................................

3-2 Real Time Data-Data Updated (Dec 12-2004)....................................19

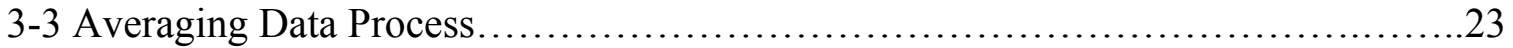

3-4 Importance of using right presets at their corresponding Pressure intervals.............24

3-5 Consequences of using very high or very low preset values-Low Interval...........25

3-6 Consequences of using very high or very low preset values-High Interval...........26

3-7 Importance of using right presets at their corresponding Flow Rate intervals...........27

3-8 Real Time Data (Jan 31-2004)-Actual data overlapping summarized data..............28

3-9 Real Time Data (Jan 31-2004)-Summarized data.................................... 28

3-10 Real Time Data (Jan 31-2004)-Actual data overlapping summarized data...........29

3-11 Real Time Data (Jan 31-2004)-Summarized data..................................29

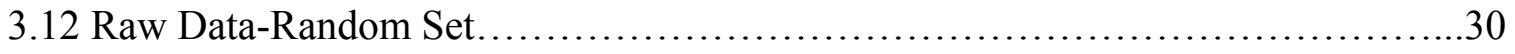

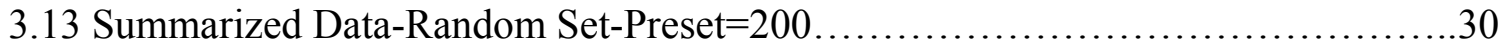

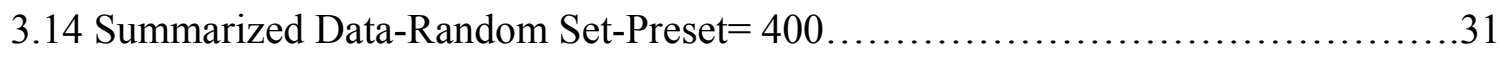

3.15 Summarized Data Selection Process ...........................................35

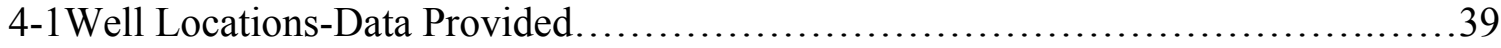

4-2 Pressure Transient Period detection.............................................40

4-3Well Test Information for Pressure vs. time and Personal Information window......41

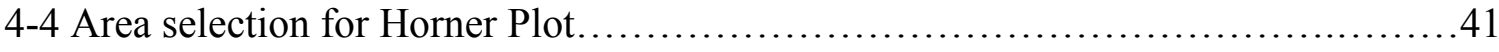

4-5 Horner Plot and Line Drawn to obtain permeability and skin factor.................42

4-6 Pressure and Flow Rate- Actual and Summarized Data (Jan 27- 05)................43

4-7 Pressure and Flow Rate -Summarized Data (Jan 27- 05)...........................43

4-8 Pressure and Flow Rate -Actual and Summarized Data (Jan 14-05) ....................44

4-9 Pressure and Flow Rate- Summarized Data (Jan 14-05) ...........................4

4-10 Pressure and Flow Rate- Actual and Summarized Data (Feb 20-05)...................45

4-11 Pressure and Flow Rate -Summarized Data (Feb 20-05)...........................45 
4-12 Pressure and Flow Rate- Actual and Summarized Data (Feb 23-05)..............46

4-13 Pressure and Flow Rate -Summarized Data (Feb 23-05).......................46

4-14 Pressure and Flow Rate- Actual and Summarized Data (March 14-05..............47

4-15 Pressure and Flow Rate -Summarized Data (March 14-05)......................47

4-16 Pressure and Flow Rate- Actual and Summarized Data (Dec 26-04)................48

4-17 Pressure and Flow Rate -Summarized Data (Dec 26-04).......................49

4.18 Pressure and Flow Rate- Actual and Summarized Data (Dec 26-04)

-Preset Changed.......................................................... 50

4-19 Pressure and Flow Rate -Summarized Data (Dec 26-04)-Preset Changed...........50

4-20 Pressure and Flow Rate- Actual and Summarized Data (March 15-05)............51

4-21 Pressure and Flow Rate -Summarized Data (March 15-05).....................51

4-22 Pressure and Flow Rate- Actual and Summarized Data (March 15-05)

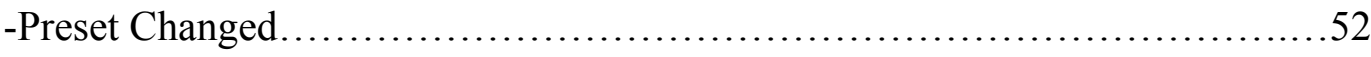

4-23 Pressure and Flow Rate -Summarized Data (March 15-05)

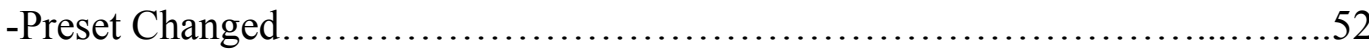

4-24 Pressure, Flow Rate, and Gas in Place Profile

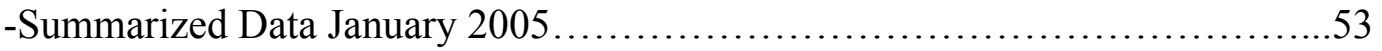

4-25 Pressure, Flow Rate, and Gas in Place Profile

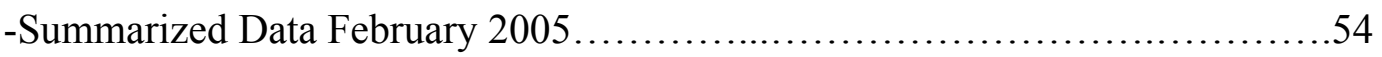

4-26 Pressure, Flow Rate, and Gas in Place Profile

-Summarized Data March 2005...........................................54

4-27 Pressure, Flow Rate, and Gas in Place Profile

-Summarized Data April 2005..........................................55

4-28 Pressure, Flow Rate, and Gas in Place Profile

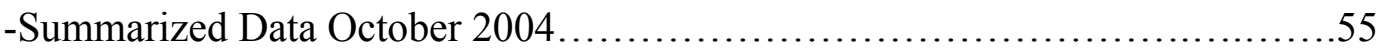

4-29 Pressure, Flow Rate, and Gas in Place Profile

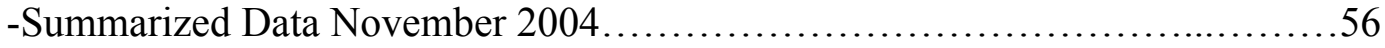

4.30 Gas in Place in Real-Time..............................................57

4-31 Pseudo Pressure, Flow Rate, and Gas in Place Profile

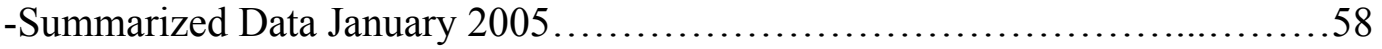

4-32 Area selection for Horner Plot-Pseudo Pressure Analysis........................59 
4-33 Horner Plot and Line Drawn to obtain permeability and skin factor

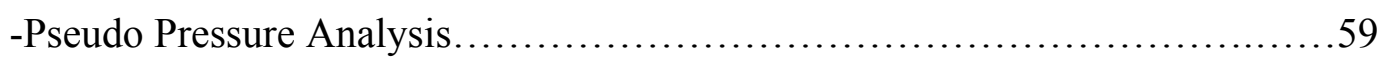

4-34 Pressure Square, Flow Rate, and Gas in Place Profile

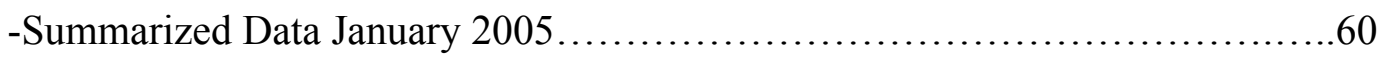

4-35 Area selection for Semi Log Plot-Drawdown Period.............................61

4-36 Semi-Log Plot and Line Drawn to obtain permeability and skin factor-Pressure

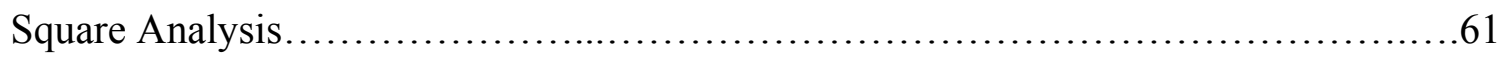

4-37 Decline Curve Analysis-Gas Produced versus time...............................62

4-38 Decline Curve Analysis-Gas Produced versus cumulative production...............63

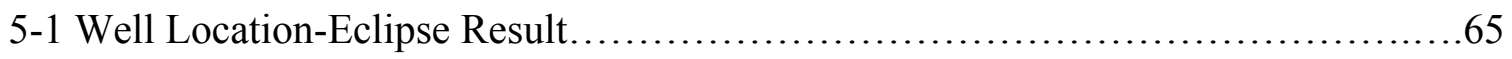

5-2 Production-Flow Rate constant- Eclipse Results.................................66

5-3 Production-Flow Rate constant-SFDA Results..................................66

5-4 Pressure Transient Period-Eclipse Results.......................................67

5-5 Pressure Transient Period-SFDA Results..........................................67

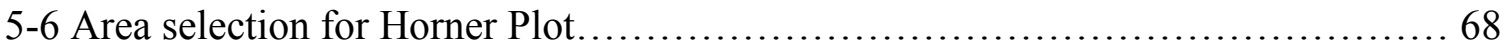

5-7 Horner Plot and Line Drawn to obtain permeability and skin factor.................68

5-8 Area selection for Horner Plot-Pseudo Pressure Analysis..............................69

5-9 Horner Plot and Line Drawn to obtain permeability and skin factor

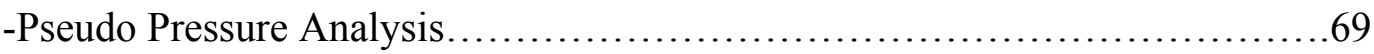

5-10 Two Pressure Transient Periods-Eclipse Results..................................70

5-11 Two Pressure Transient Periods-SFDA Results.................................70

5-12 Area selection for Horner Plot-Pseudo Pressure Analysis......................... 71

5-13 Horner Plot and Line Drawn to obtain permeability and skin factor................71 


\section{Chapter 1}

\section{Introduction and Motivation}

The process of monitoring real time data represents an innovative approach for data acquisition. Production fields that consist of the hardware that monitor high frequency data from downhole and transfer such data to the engineering desktop are best known as Smart Fields or Intelligent Wells.

The concept of obtaining data in real time is related to the time step at which data is measured and read in a computer database. In most cases several parameters such as

pressure and flow rate are monitored every 10-15 seconds. The fact that data is coming into the system at such a small interval of time creates an enormous amount of data in a few months and even in weeks. For example, a week of data monitoring will have around forty thousand values, and one month will consist of a hundred and eighty thousand data points. Similarly, one year will contain about three million measurements [1]. The extremely large volume of data requires special handling and interpretation techniques which will be part of the scope of this investigation.

The simple definition of real time includes the measurements made, the succeeding analysis of those measurements, the evaluation of such an analysis, the process of decision making, and the changes to the system after taking actions.

The benefit of having intelligent wells is related to efficiency in terms of reaction time before or during a particular event takes place. The sooner the engineers react to a particular incident, the better the production optimization will be [1].

Engineers face different problems when using real time data for real time data optimization. Such problems include the quality of the data, the transport of huge amounts of data, and that fact that no engineering packages are capable of handling high frequency data [1]. 
The continuously monitored data provides more information to the engineers than the sporadic measurements, which helps them to understand the behavior of the field in a better and more efficient matter.

Currently, there are a lot of fields that are mostly offshore that can measure pressure and flow rate at different time intervals ranging from seconds to minutes. The enormous amount of data created in a month makes the manipulation of the data very difficult. In most cases, most of the real time data remains unused in the high frequency database. Usually, the high frequency data is used only for pressure transient analysis.

There are different publications about smart fields and intelligent wells focusing primarily on the hardware used to obtain data in real time. However, there is little related to the use of these real time measurements to create value. In order words, once the data is in the system; what can be done with such an amount of data? How can the huge amount of data be handled? What can be obtained from the real time measurements? [1]

\subsection{Statement of the Problem}

The premise of this work is building the first block for Smart Field Data Analysis that will serve as a bridge in the creation of a more complex computer program that performs reservoir engineering analysis on real time data. During this study; different problems will be encountered such as handling a vast amount of data, detecting pressure transient periods in real time, and creating value from the high frequency data once the information has been summarized. A computer program capable of solving the problems just described will be developed and tested using actual real time data generated from the storage fields. In addition, the software will be validated using generated data from a simulator and tested for different scenarios such as sudden increases in pressure. 
1.2 Schematic of the Program

\section{Schematic of the Software}

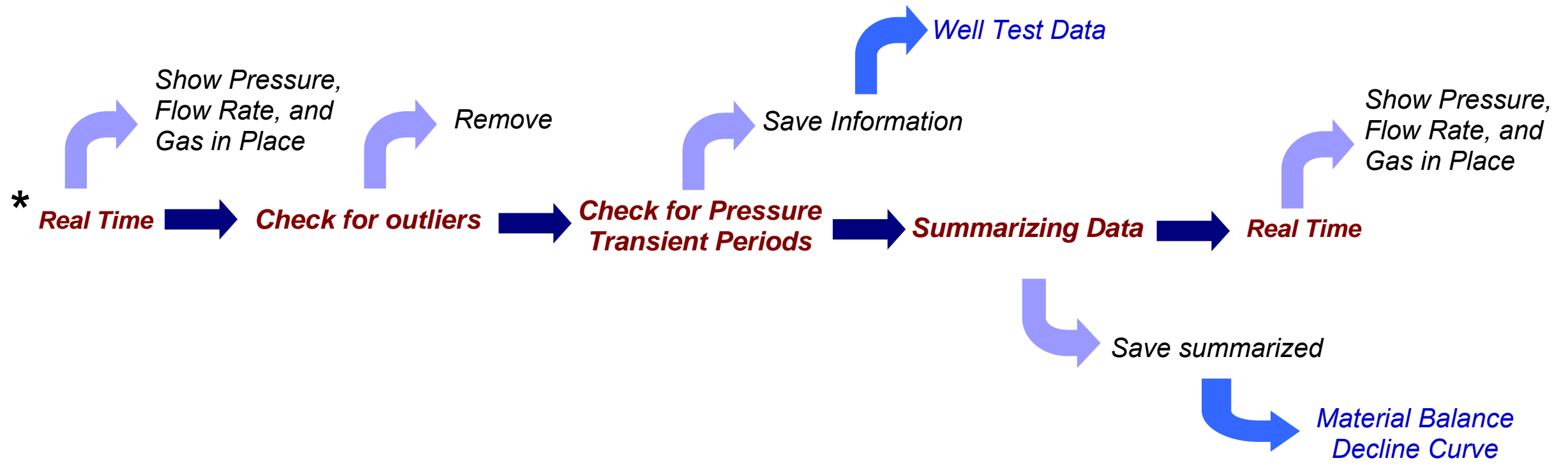

Figure 2.1: Schematic of the Program

* Starting Point 


\section{Chapter 2}

\section{Theoretical background}

\subsection{Reservoir Management}

\subsubsection{Smart Fields}

An innovative way of reservoir management consists on intelligent well or smart fields. The main function of the Smart fields is to automate as many tasks as necessary to increase the net production of an asset and to reduce costs. Smart fields are composed usually of pressure, flow rate, and temperature gauges that measure data in real time [1].

\subsubsection{Real Time Data}

The meaning of Real Time in the petroleum industry is often misunderstood by the assumptions of reacting within a short period of time to a certain situation. A process that deals with Real Time data is an operation in which the reaction and time of a particular event is shorter than the maximum delay that is permitted and the task is performed before the system becomes unstable. For instance, a system capable of shutting in the well before it is damaged is one example of this type of operation [1]. 


\subsubsection{High Frequency Data}

The process of integrating real time data into a reservoir management system and converting such data into value is a challenging task for the engineers today. The transfer of the real time data from down-hole wells to the desktops in a clean and smooth manner represents the bottleneck of handling high frequency data.

The fact that real time data comes into the database at a very high frequency characterizes another obstacle in reservoir management. The large amount of data does not allow the engineers to verify inconsistency in the data. As a result, intelligent reservoir management utilizes alarm systems that notify the engineers of any discrepancy from the data or any critical condition present in the reservoir.

Neural networks and genetic algorithms represent very important tools to handle the huge amount of data that real time measurements provide. A neural network learns from the data collected and identifies underlying relationships. When such underlying relationships have been established, the neural network can be used to make certain predictions allowing the engineer time to react in case anything different from the predicted trend occurs [1].

\subsubsection{Real Time Data Acquisition}

A high frequency gathering system known as SCADA (Supervisory Control and Data Acquisition) has been used by many companies in the oil industry. The system basically stores real time data and collects it for the engineers to analyze it. The process of data acquisition consists of data cleansing, data aggregation, and data integration [2].

\subsubsection{Data Cleansing}

Data cleansing is essential in handling high frequency data. Outliers and noise will always be present in the data under any kind of conditions.

Outliers are single data points lying clearly away from the real trend and noises are data points scattered around the real trend [1]. 
The cleansing process consists of removing both outliers and noises from the data in order to make it more smooth and easier to analyze.

\subsubsection{Data Aggregation}

The process of data aggregations consists of aggregating the data to bigger time increments (from 15 seconds to 15 minutes) for better visualization. Visualizing the data with a very small time increment would be hard for analysis. Thus, data needs to be aggregated, except in certain conditions, such as during shout in period in which high frequency data points are necessary for pressure build up analysis [1].

\subsubsection{Data Integration}

Once the data has been cleaned and aggregated, it needs to be validated in different applications using different time increments. The process of data integration consists of integrating the data in different time increments to be stored in a database for future analysis [1].

\subsubsection{Models and Detection}

An aspect of engineering analysis is the continuous optimization of data by developing a mathematical model that best describes the process. Conversely, in petroleum engineering, such a procedure is very difficult to achieve since conditions routinely change during production. However, with the increasing amount of available parameters it is possible to model the performance of the reservoir. The use of real time data along with accessible reservoir information is used to develop a mathematical model that describes reservoir behavior and alerts engineers for anomalous situations [1]. 


\subsubsection{Detection}

Simple alarms can be used to detect any variations in some calculated parameters. Examples of calculated parameters can be water cut, gas oil ratio, and voidage ratio.

An increase in water cut represents water breakthrough. The gas oil ration can be used to determine an extremely high gas production when the gas production exceeds the relationship between gas and oil ratio. The voidage ratio is used to alert an excessive water injection in some locations of the reservoir [1].

\subsubsection{Decline Curve Analysis}

The engineers decide the trend of the decline curve which provides the foundation for the alarm system. The actual program compares the oil or gas production with the expected one. If a certain amount of data points start falling off the trend, the system will activate the alarm to indicate that something unusual is taking place [1].

\subsubsection{Material Balance with Interference}

Another more complex model that is used to trigger the alarm system is material balance. The material balance calculates static pressure as well as flow rate of either oil and water or of gas and water. The available software basically receives production data and automatically computes static reservoir pressure as data is feeding the system. The upper and lower boundary for the static pressure is set and the injected volumes are regulated such that the static reservoir pressure remains within the two limits. This helps to prevent excessive injection into single compartments and avoids quick depletion while maintaining the pressure of the reservoir [1]. 


\subsubsection{Reservoir Simulation}

Another mechanism that can be used in handling real time data and reacting under abnormal situations is the prediction of a reservoir simulation. The actual oil or gas production rate is constantly compared to the predicted data. If the real production deviates from the expected trend, the alarm system will alert the engineers [1].

\subsubsection{Data-Driven Tools}

Data driven tools such as neural networks have gained importance in the process of gathering data in real time by learning from stored data to predict and optimize processes establishing a nonlinear relationship between input and output parameters. The learning process basically finds relationships an engineer would never find. One of the applications of using neural networks is the rate of allocation back to the individual wells in real time [1].

\subsection{Long Term Data Analysis}

\subsubsection{Outlier Removal}

As explained in the data cleansing section, outliers are data points that lie clearly away from the rest of the data which causes discontinuity and creates singularity between two successive data points. The presence of an outlier creates the first singularity and the second singularity is the result of a sudden decrease or increase of a data point jumping back to the trend.

The process of removing outliers starts by selecting a threshold that will determine the values that will be considered as outliers. The selection depends on how far a data point has to be from the trend. Generally, the magnitude of the threshold depends on degree of changes in pressure and in the resolution of the instrument used to monitor data. 
Normally, 5 psi is used as a threshold for outlier removal. Due to the fact that data usually has outliers bigger than the threshold, the ones with the largest magnitude are removed first and then the others are removed until the ones with a magnitude of about 5 psi are gone[10].

\subsubsection{Denoising}

The process of denoising consists of reducing the scattering and the fluctuation in the data points to get the most representative section of the data. The most known method of denoising the data is the wavelet thresholding method introduced by Donoho and Johnstone. Data values are compared to a certain value of threshold; the wavelet detail having a magnitude value lesser than a threshold value are set equal to zero. This process is also known as wavelet shrinkage. Two thresholding methods best known as the hard and soft threshold method can be used. The condition for the hard thresholding method is the following [10]:

$$
\begin{aligned}
d_{j, k}^{\text {hard }}=d_{j, k} & \left|d_{j, k}\right|>\lambda \\
0 & \text { otherwise }
\end{aligned}
$$

where the $d j, k$ represents the detail signal or data points and $\lambda$ is the threshold. On the other hand, the principle for the soft thresholding method is:

$$
\begin{array}{cc}
d_{j, k}^{\text {soft }}=d_{j, k}-\lambda & d_{j, k}>\lambda \\
d_{j, k}+\lambda & d_{j, k}<-\lambda \\
0 & \left|d_{j, k}\right| \leq \lambda
\end{array}
$$

After several experiments working with different kinds of data, it was concluded that the benefit of the hard thresholding method consists of preserving signal sharpness and the advantages of the soft thresholding method are related to the smoothness of noisy features. A new method that combines both the hard and soft threshold method was developed. Such a method is known as the hybrid thresholding method. The hybrid 
method combines the benefit of the soft and the hard method to obtain a more accurate representation of the data. The criterion for the hybrid method is the following:

$$
\begin{array}{cl}
d_{j, k} & \left|d_{j, k}\right|>\lambda \text { and }\left|d_{l, k}\right|>\lambda \\
d_{j, k}^{\text {hybrid }}=\quad & d_{j, k}>\lambda \quad \text { and }\left|d_{l, k}\right|<\lambda \\
d_{j, k}-\lambda & d_{j, k}<-\lambda \quad \text { and }\left|d_{l, k}\right|<\lambda \\
0 & \left|d_{j, k}\right| \leq \lambda
\end{array}
$$

where 1 represents the decomposition used to verify sharp fluctuations.

\subsubsection{Transient Identification}

Drastic changes in pressure can be used in some cases to determine changes in flow rate. Such changes are viewed as singularities in the data. Thus, the wavelet modulus that specifies the singularities of data points close to each other can then be utilized to determine the moments when changes in flow rate occur.

Similar to the process of detecting outliers, a threshold value has to be assigned for the detail signal or data points in order to identify singularities in the data. The approach of selecting the threshold is related to how big the pressure difference has to be in order to be considered as the beginning of a new transient.

In order to confirm that the singularities detected are the result of a new transient and not of local variations, the slope of the data points close to the actual signal need to be checked. First of all, the slope of the signal after the singularity must be higher than the slope of the threshold. Then, the absolute pressure difference between the slope after and before the singularity needs to be high enough to confirm a discontinuity in the trend of the data. In some cases, when the difference between the two slopes is greater than half of the slope threshold, the detected data point is considered to be a singularity [10]. 


\subsubsection{Data Reduction}

The amount of real time data collected is very large. Since data is coming from permanent down-hole gauges every 10 to 15 seconds, after one year there will be approximately more than 3 million data points per well. Dealing with such an amount of data is very difficult; thus, data should be reduced to a reasonable size. The thresholding method is one of the methods used for data reduction. In this method, a preset value for any parameter such as pressure is established. The process then compares every data point with the preset value. If the data point is higher than the preset value, such a signal is collected. On the other hand, if the pressure value is less than the preset value, the value is ignored. Small changes in pressure might become large changes after a period of time. If the pressure values are not collected because they are less than the values of the preset pressure, after a certain period of time, such changes might be large and mistakenly ignored. Therefore, a preset for time is also taken into consideration when dealing with data reduction. The process then compares pressure values with the preset pressure value and the time is constantly compared to a preset value of time. After the time is greater than the preset time, the value of pressure and the time at that moment are collected and the time is reset until the next preset for time is reached [10]. 


\subsection{Permanent Downhole Equipments used in Smart Fields}

Permanent monitoring systems are used in the petroleum industry to collect and to gather real time data. The procedure of data acquisition is very important for good reservoir monitoring and production techniques, since it provides the engineers with real time data to make timely and accurate decisions. The new developments on this area are related to the types of sensors placed downhole to monitor the data. The most noticeable advance on new gauges is the change from wireline to wireless sensors, as well as a switch in the material; for instance from strain or quartz to fiber optic.

New technology in sensors does not necessarily mean that they meet the demand of the oil industry nowadays. For example, in terms of accuracy (defined as the closeness with which a measurement agrees with the standard), the fiber optic sensor and the electronic quartz share a common accuracy of about 0.001 psia. Nevertheless, transmission rates are higher than the electronic quartz systems.

The family of sensors used in the process of real data acquisition can be divided into three kinds: wireline electronic gauge, wireline fiber optic and wireless sensors. In general, the wireline electronic gauge belongs to the first generation of permanent downhole equipment and can be of three different materials: strain, quartz, or sapphire. The wireline fiber optic corresponds to a newer technology and its main characteristic is its high reliability and high transmission rates. The wireless gauges belong to the latest technology in terms of real time data acquisition. Its principal advantage is its high reliability as well as the elimination of any cable or electronic downhole tool [18]. 


\subsection{Real Time Decision Making While Drilling}

Some researchers have proposed a real time optimal control strategy in order to identify, detect and predict drilling events in real time. The aim will be to make the proper decision in terms of operational drilling performance, initial well completion design, well productivity, and optimal value creation [3].

\subsubsection{Real Time Drilling and Completion Modeling}

The real time modeling strategy is used to simulate the drilling environment and reservoir conditions in order to improve drilling performance, detect irregular situations, redesign well construction, well completions, and well dynamics [3].

\subsubsection{Abnormal Situation Detection and Management}

Abnormal situations and detection consists of detecting and correcting problems that take place during the drilling process. The aim of this strategy is to perform these tasks within a short period of time that will permit safe operations. The time frame for real time abnormal situation management varies from a few seconds to a few minutes and from a few minutes to a few hours depending on the drilling process [3]. 


\subsection{Routine Reservoir Surveillance with Neural Networks and Simplified Reservoir Models}

As it has been explained before, Intelligent Well completion creates an enormous amount of data which is very difficult to handle.

An innovative approach to detect discrepancies from the expected trends through a process of learning data mining system has been developed.

Reservoir surveillance plays an important role for real time decision in Smart Fields once several problems encountered in the Smart Field industry have been solved. Most of such problems are outline below [13]:

1. Data overload and Data Delivery

2. Inability to deliver proper data in a timely fashion

3. Creating values from the real time measurements

4. Emerging technology for the process of decision making

5. Forecasting actual reservoir performance

6. Identifying abnormal situations as soon as possible

7. Providing information regarding the cause of discrepancy in the data

8. Using all information to recognize unexpected situations

\subsubsection{Decision Making Process}

The decision making process consists of three levels of reservoir management: operation support, development engineering, and field development planning.

Operation support is supposed to work in real time on well intervention level and the time of reaction frame between the real actual time and a few days.

Development engineering decisions involve interactive work with reservoir observation models to any intervention needed with a time frame (from several days to weeks). Field development decisions are carried out on a field level during the whole life of the reservoir [13]. 


\subsubsection{Event detection}

Different reservoir engineering techniques such as decline curve analysis and fractional water-cut are included to generate trends and predictions from reservoir surveillance information. Some of the results are used to predict Material Balance with Interference. The expected trend as well as the updated information is calculated from models at different scenarios such as suddenly increases and decreases in pressure and flow rate.

The expected trend models are continuously updated using the real time data alerting the engineers for any discrepancy through alarms and notification systems. Usually a deviation of about $10 \%$ of the expected trend is used to notify engineers that a well might need intervention.

One of the challenging for this type of reservoir surveillance is the detection of trend violation in a timely manner. Therefore, Data Mining models have been developed in order to account for this task.

The trained data comes from the combination of reservoir surveillance, some static parameters, historical data, and models that automatically place records with analogous characteristics into similar groups, such as wells with similar performance [13]. 


\section{Chapter 3}

\section{Methodology}

A computer program that performs data analysis to be used in Smart Fields has been created using Visual Basic 6.0 and its methodology will be explained throughout this chapter. Chapter 3 will describe how the software has been created with a detailed explanation of the approach used for its development. Real time data provided by Columbia Gas Transmission Corporation was used to evaluate the software and to test it during several types of production analysis such as during injection and production periods, and during pressure transient periods; Chapter 4 will focus on examples and results after using the real time data provided by Columbia Gas Transmission Corporation and running the software. The computer program has been named SFDA (Smart Field Data Analysis).

\subsection{Real Time Measurement}

Since data is coming into the system in real time, a computer program that is capable of reading certain parameters like pressure and flow rate has been created. The software allows the user to observe pressure and flow rate instantaneously while data is available in real time.

As data is feeding the system, SFDA takes every value and plots it in order to allow the user to examine the data that is coming from the production fields. Any drastic change such as a sudden increase in pressure or a drastic decrease in any parameter can be seen by the user and the appropriate actions can be taken right away.

The most common way to read data from a computer program is by using arrays in which the data that is being read fills the arrays. Since data is always coming, there will be a moment when the array will be full and no more data will be filling it. 
Visual Basic 6.0 allows data to be placed in a grid while data is being read by the program. In effect, SFDA reads data and adds a row to the grid. As data is being read, it keeps adding rows. The operation is repeated until a maximum of 500 data points (information of two hours of production) is reached. When this happens, the software will start deleting the first row and new rows will keep adding to the grid, permitting the program to read data indefinitely.

As data is read, the program places it in the grid and new rows are added as new data points are entering the system. See the example below assuming that the tables contain actual data:

\begin{tabular}{|c|c|}
\hline $\begin{array}{c}\text { Pressure } \\
\text { (psia) }\end{array}$ & $\begin{array}{c}\text { Time } \\
\text { (sec) }\end{array}$ \\
\hline 700 & 100 \\
\hline 705 & 115 \\
\hline 710 & 130 \\
\hline 715 & 145 \\
\hline 720 & 160 \\
\hline 720 & 175 \\
\hline 725 & 190 \\
\hline
\end{tabular}

Table 3.1: Random RealTime Data-Step 1

\begin{tabular}{|c|c|}
\hline $\begin{array}{c}\text { Pressure } \\
\text { (psia) }\end{array}$ & $\begin{array}{c}\text { Time } \\
\text { (sec) }\end{array}$ \\
\hline 700 & 100 \\
\hline 705 & 115 \\
\hline 710 & 130 \\
\hline 715 & 145 \\
\hline 720 & 160 \\
\hline 720 & 175 \\
\hline 725 & 190 \\
\hline 735 & 205 \\
\hline
\end{tabular}

Table 3.2: Random RealTime Data-Step 2

\begin{tabular}{|c|c|}
\hline $\begin{array}{c}\text { Pressure } \\
\text { (psia) }\end{array}$ & $\begin{array}{c}\text { Time } \\
\text { (sec) }\end{array}$ \\
\hline 700 & 100 \\
\hline 705 & 115 \\
\hline 710 & 130 \\
\hline “ & “ \\
\hline “ & 175 \\
\hline 720 & 190 \\
\hline 725 & 205 \\
\hline 735 & 220 \\
\hline 740 & \\
\hline
\end{tabular}

Table 3.3: Random RealTime Data-Step 3 
When the grid reaches its maximum capacity of 500 data points (around 2 hours) SFDA will start deleting the first row and new rows will be added which allows the software to run on and on. Before deleting any value, the program will save all information in a folder previously selected where the engineers will be able to access such data later on for analysis.

\begin{tabular}{|c|c|}
\hline $\begin{array}{c}\text { Pressure } \\
\text { (psia) }\end{array}$ & $\begin{array}{c}\text { Time } \\
\text { (sec) }\end{array}$ \\
\hline 700 & 100 \\
\hline 705 & 115 \\
\hline 710 & 130 \\
\hline$“$ & “ \\
\hline “ & “ \\
\hline 720 & 175 \\
\hline 725 & 190 \\
\hline 735 & 205 \\
\hline 740 & 220 \\
\hline
\end{tabular}

Table 3.4: Random RealTime Data-Deleting First Row Process

\begin{tabular}{|c|c|}
\hline $\begin{array}{c}\text { Pressure } \\
\text { (psia) }\end{array}$ & $\begin{array}{c}\text { Time } \\
\text { (sec) }\end{array}$ \\
\hline 705 & 115 \\
\hline 710 & 130 \\
\hline 715 & 145 \\
\hline$،$ & ، \\
\hline$،$ & 190 \\
\hline 725 & 205 \\
\hline 735 & 220 \\
\hline 740 & 235 \\
\hline 750 &
\end{tabular}

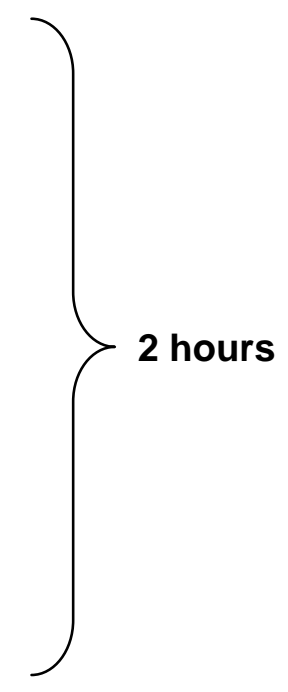

Table 3.5: Random Real-

Time Data-Updated

Process 
Once the data is located in a grid, the program plots the available parameters, such as pressure and flow rate versus time. This is being done while the data from the production fields is coming into the system. See the next examples:

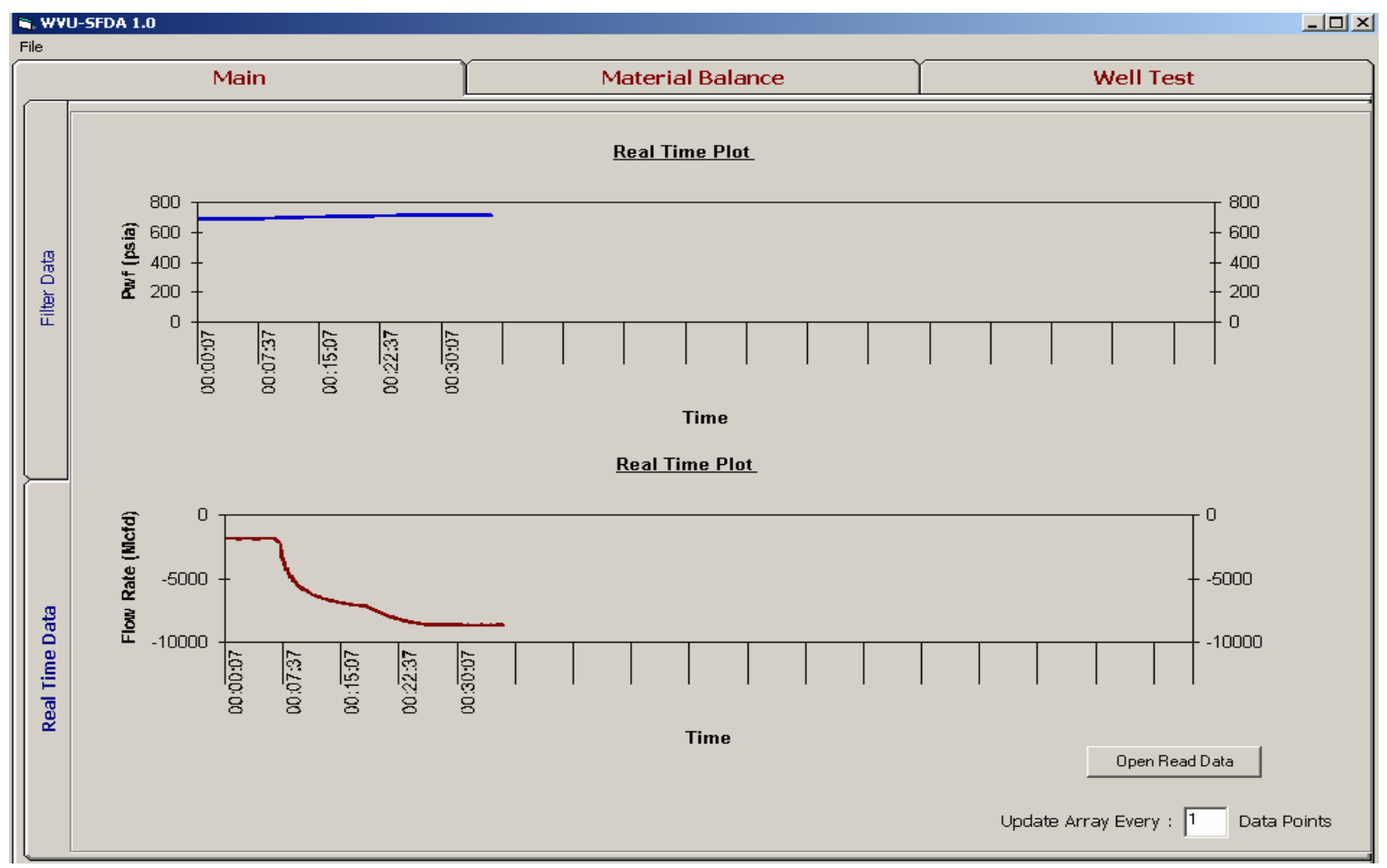

Figure 3.1: Real Time Data (Dec 12-2004)

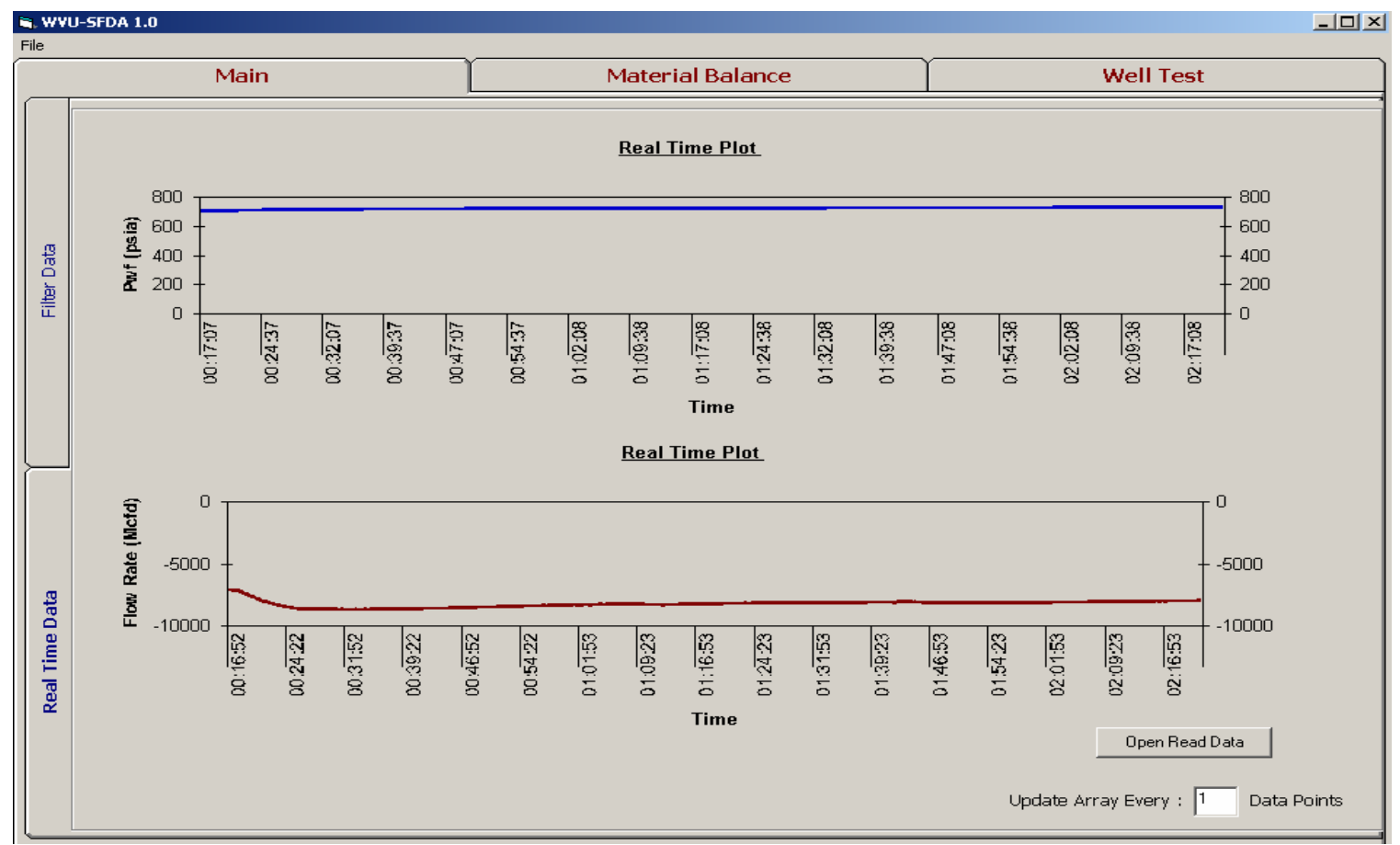

Figure 3.2: Real Time Data-Data Updated (Dec 12-2004) 


\subsection{Pressure Transient Analysis and Outlier Removal Process}

SFDA has the capability of detecting pressure transient periods, saving all information in a folder, and preparing the data for the user to analyze. The software will decide whether the sudden increase in pressure is due to a pressure transient period or to an outlier. If the program decides that the transient is because of an outlier, it will remove it from the system.

When the pressure increases to about $30 \%$ of the average of the last five values previously recorded, the program will start comparing the actual values with such an average. If a sudden increase in pressure value takes place, the software will read the next value and compare it to the average of the last five values. If the next value is still very high, the program will repeat the process and will keep going on about three more times until the program has decided that a transient period has taken place. When this happens, the program will start saving pressure and time information in a folder. Another window will show that a file is being created and is opened for the process of the pressure transient analysis. Information coming into the system will be read in real time, sent to a file that keeps all information named "Raw Data," and the information related to the transient identification will be saved to a file named "Well Test." As soon as the pressure comes back down, the software will close such a file and the information will be ready for the user to analyze.

Conversely, if a very high increase in pressure takes place in which such a value is greater than the average of the last five points, and the program compares the next value with the average of the last five points resulting in a discontinuation of the pressure build up process, the program will immediately detect that an outlier is present in the data and will remove it from the system. No information related to well testing analysis will be saved as a result of this occurrence. 
The following example will best illustrate the methodology used for pressure transient analysis:

\begin{tabular}{|c|c|}
\hline Pressure & Time (sec) \\
\hline 51 & 100 \\
\hline 48 & 115 \\
\hline 53 & 130 \\
\hline 50 & 145 \\
\hline 48 & 160 \\
\hline 700 & 175 \\
\hline 701 & 190 \\
\hline 702 & 205 \\
\hline 704 & 220 \\
\hline
\end{tabular}

Table 3.6: Real-Time DataPressure Transient Detection

\begin{tabular}{|c|c|}
\hline Pressure & Time \\
\hline 51 & 100 \\
\hline 48 & 115 \\
\hline 53 & 130 \\
\hline 50 & 145 \\
\hline 48 & 160 \\
\hline 700 & 175 \\
\hline 55 & 190 \\
\hline 52 & 205 \\
\hline 58 & 220 \\
\hline
\end{tabular}

Table 3.7: Real-Time Data-Outlier Detection

The information table on the left shows a pressure build up and a continuation in the process. The program reads the first very high value (700 psia) and compares it to the average of the five previous values which correspond to 50 psia. The next value (701 psia) is again compared to the value of 50 psia and again is a very high value. The same operation keeps repeating until the program detects a transient period in the data and starts saving such data values in a folder containing build up data.

On the other hand, there might be a situation where the program detects a very high increase in pressure value but the data does not follow that pattern. The information table on the right shows a case where an outlier is present in the data instead of a transient period. When the program reads the value of 700 psia and compares it to the average of the last five points, the program will repeat the same operation with the following value. If the next value ( 55 psia from the table) is not greater than the average of the previous five values already calculated, the program automatically deletes the value that will contain a discontinuity in the pattern of the data. The program separates outliers from a pressure transient analysis. 
A similar operation will take place for flow rates; whenever a value for flow rate is greater than the average of the last five values, the program will delete such a value as long as the next value does not follow the trend of the values about to be eliminated. For flow rate, no consideration of a transient period will be taken since only pressure values are used to detect valuable information about well test analysis. Therefore, no file will be created or opened when changes and new patterns in flow rate occur. When a value is deleted, all information related to such a value is also removed. For instance, if the value of 700 psia presented in the information table on the right is identified as an outlier, the flow rate and time corresponding to such a value will be eliminated from the system as well. 


\subsection{Averaging High Frequency Data While Preserving its Integrity}

The process of averaging the data consists of the use of preset values for $\Delta \mathrm{P}$ and $\Delta \mathrm{Q}$ to summarize the high frequency data while keeping its integrity.

Preset values for $\Delta \mathrm{P}$ and $\Delta \mathrm{Q}$ represent the minimum difference at which pressure and flow rate should be recorded. Pressure and flow rate are recorded when the changes in such parameters $(\Delta \mathrm{P}$ and $\Delta \mathrm{Q})$ are higher than the maximum presets $\Delta \mathrm{P}$ Preset and $\Delta \mathrm{Q}$ Preset. Every value for pressure and flow rate is compared to a $\Delta \mathrm{P}$ Preset and $\Delta \mathrm{Q}$ Preset respectively to determine whether a value should be recorded or discarded.

The following flow chart summarizes such a process:

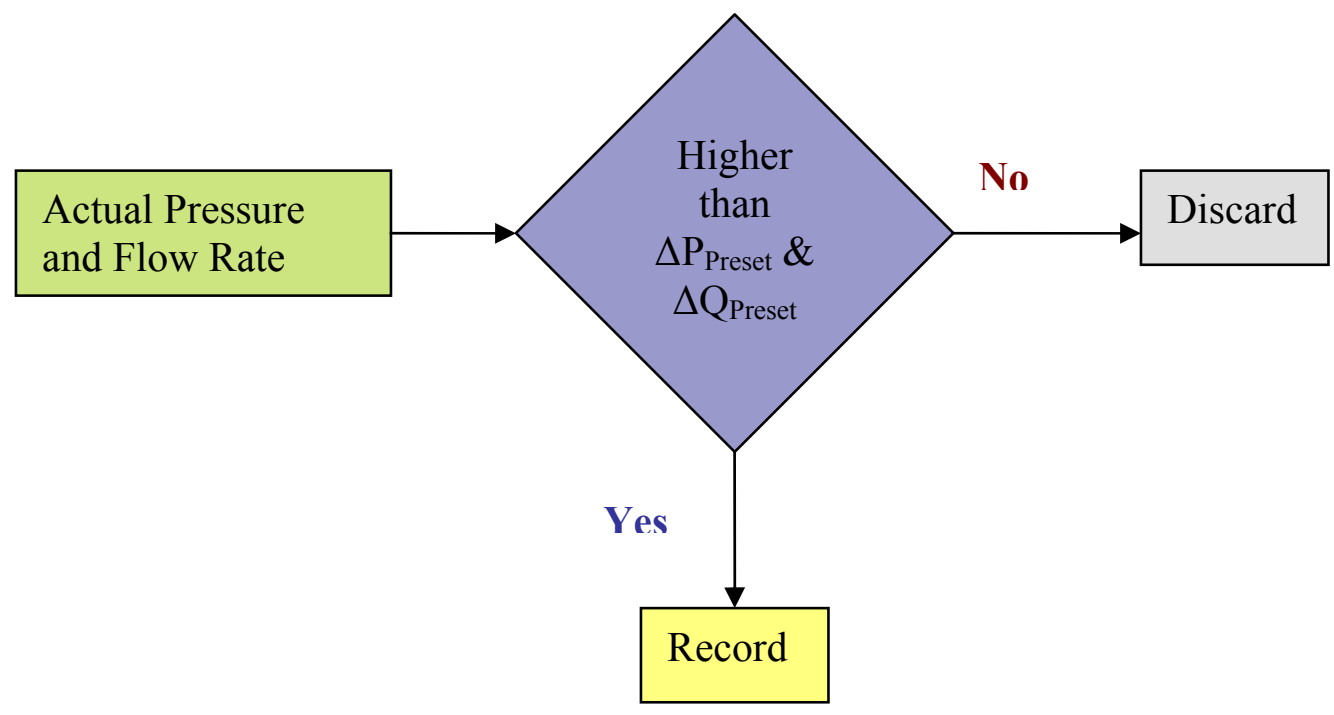

Figure 3.3: Averaging Data Process

$\Delta \mathrm{P}_{\text {Preset }}$ and $\Delta \mathrm{Q}$ Preset for different intervals of values of pressure and flow rate need to be determined. Two intervals of pressure values (e.g. 100 psia-200 psia) might require a certain value for $\Delta \mathrm{P}$ Preset (e.g. 1 psia) in order to obtain a set of summarized data with few data points and a plot very similar to the actual one. However, higher intervals of pressure values (e.g. 1000 psia-2000 psia) might require a greater value for $\Delta \mathrm{P}$ Preset (e.g. 12 psia) to obtain a few data points again and a plot very similar to the actual one. 
Pressure Intervals:

100 psia

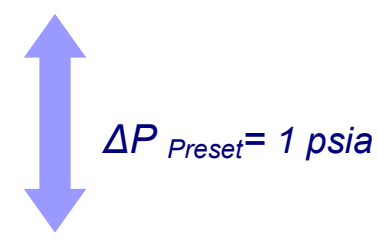

200 psia

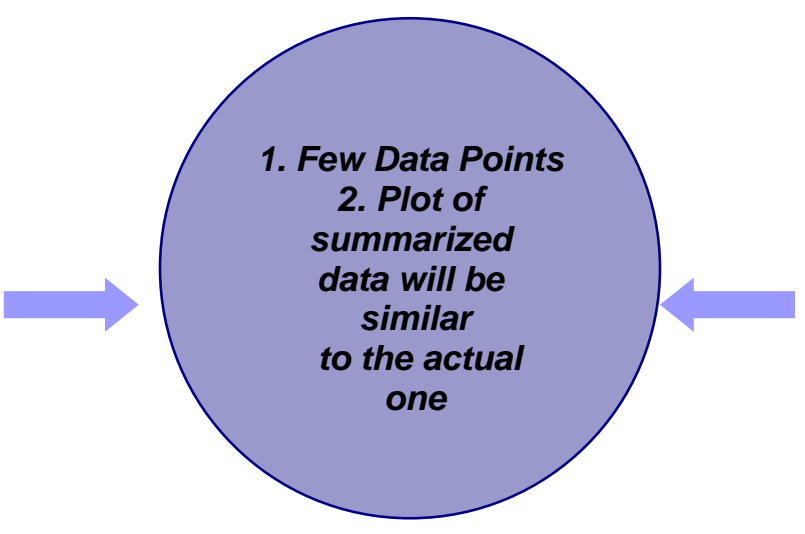

Pressure Intervals:

1000 psia

$\Delta P_{\text {Preset }}=12 p$ sia

2000 psia

Figure 3.4: Importance of using right presets at their corresponding pressure intervals

If a high $\Delta \mathrm{P}_{\text {Preset }}$ value (e.g.12 psia) is used for a low interval of time (e.g.100 psia-200 psia), the set of summarized data will contain few data points but its plot will not be similar to the actual one. On the other hand, if a low $\Delta \mathrm{P}$ Preset value (e.g.0.005 psia) is used for such an interval of time, the set of summarized data will have a plot of summarized data very similar to the actual one but the average data will contain a lot of data points. 
Pressure Intervals:

100 psia

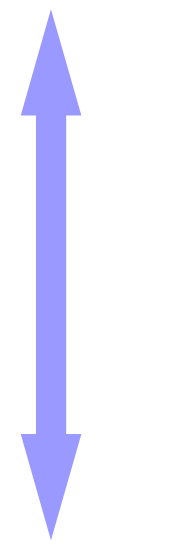

High Preset
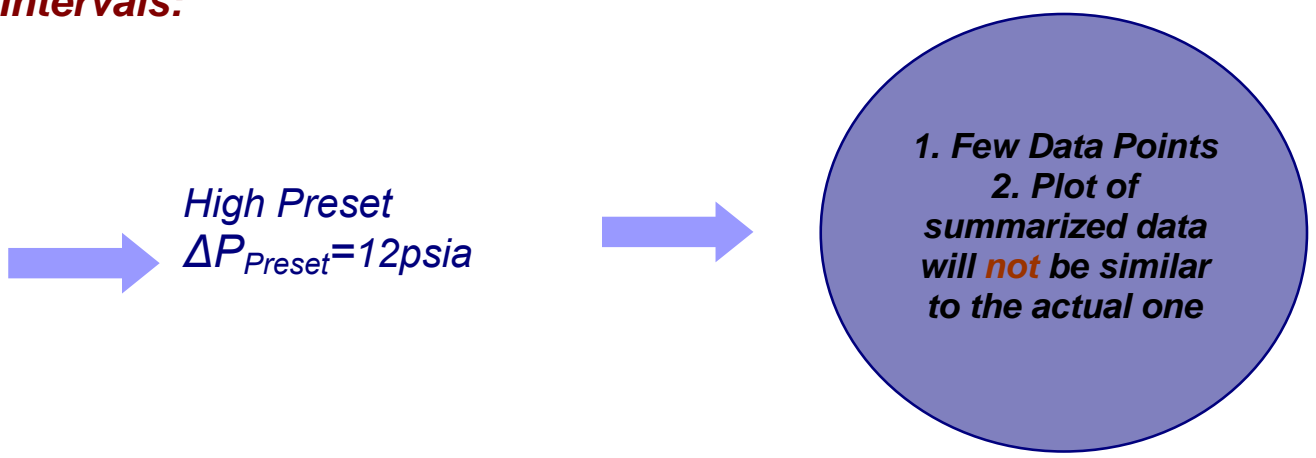

200 psia

\section{Low Preset}

$\Delta P_{\text {Preset }}=0.005$ psia

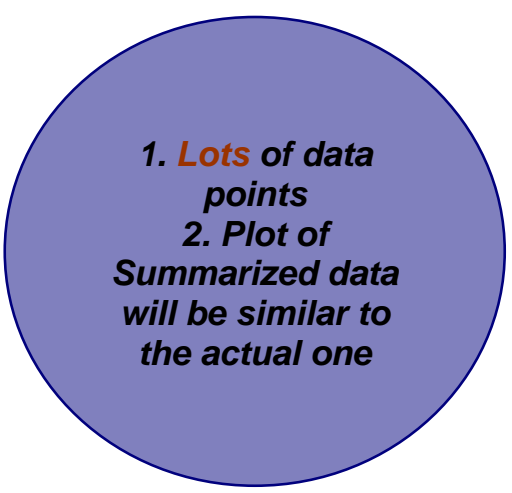

Figure 3.5: Consequences of using very high or very low preset values-Low Interval

For higher intervals of pressure value, the same methodology is applied. If a very high $\Delta \mathrm{P}_{\text {Preset }}$ value (e.g.50 psia) is used for a higher interval of time (e.g.1000 psia-2000 psia), the set of summarized data will contain few data points but its plot will not be similar to the actual one. Conversely, if a much lower $\Delta \mathrm{P}$ Preset value (e.g.1 psia) is used for such a high interval of time, the summarized data will have a plot of summarized data very similar to the actual one but the average data will contain a lot of data points. 


\section{Pressure Intervals:}

1000 psia
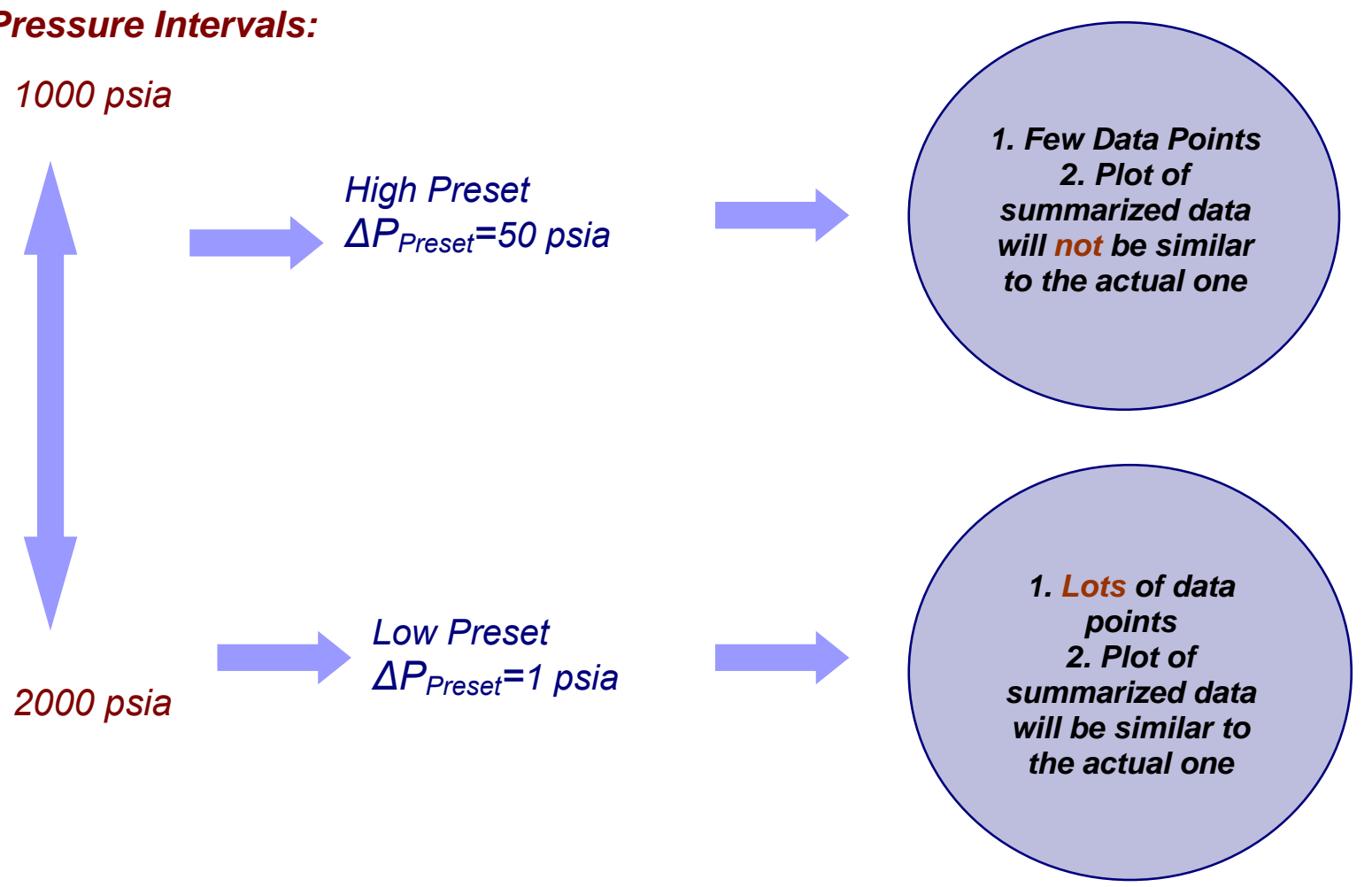

Figure 3.6: Consequences of using very high or very low preset values-High Interval 
The same principle already explained applies for flow rate data in which the proper $\Delta Q_{\text {Preset }}$ values should be used for the right interval of flow rates.

$1000 \mathrm{Mcf}$

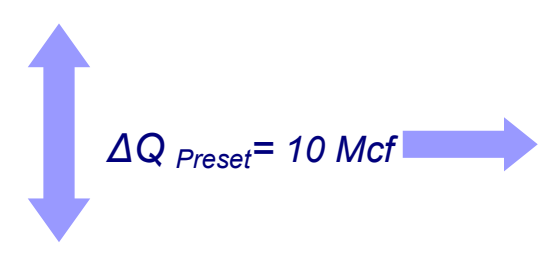

2000 Mcf

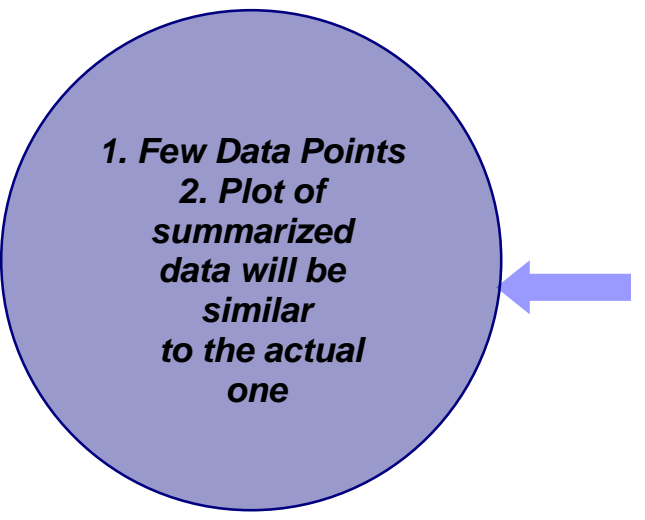

20000 Mcf

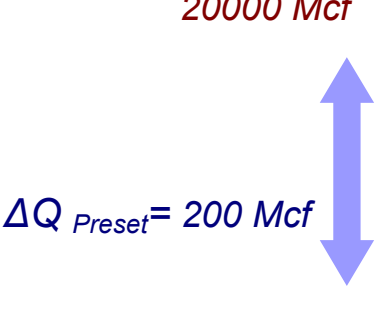

$30000 \mathrm{Mcf}$

Figure 3.7: Importance of Using Right Presets at their corresponding parameter intervals-Flow Rate

A methodology for summarizing the flow rate and pressure data will be explained in detail in which the appropriate $\Delta \mathrm{P}$ Preset and $\Delta \mathrm{Q}$ Preset values for different pressure and flow rate intervals will be obtained. 
The two figures below show a case where pressure and flow rate are plotted. Every figure has two plots (pressure vs. time at the top and flow fate vs. time at the bottom). The first figure is actually a case where the values of $\Delta \mathrm{P}$ Preset and $\Delta \mathrm{Q}$ Preset were very small in which the red line represents the actual data and the blue line represents the summarized one. As it can be seen, the red line overlaps the blue one. Figure 2 is the same as Figure 1, where only the summarized data is shown. Even though the plots are very similar, the summarized data contains lots of data points after using small values of $\Delta \mathrm{P}_{\text {Preset }}$ and $\Delta Q_{\text {Preset. }}$

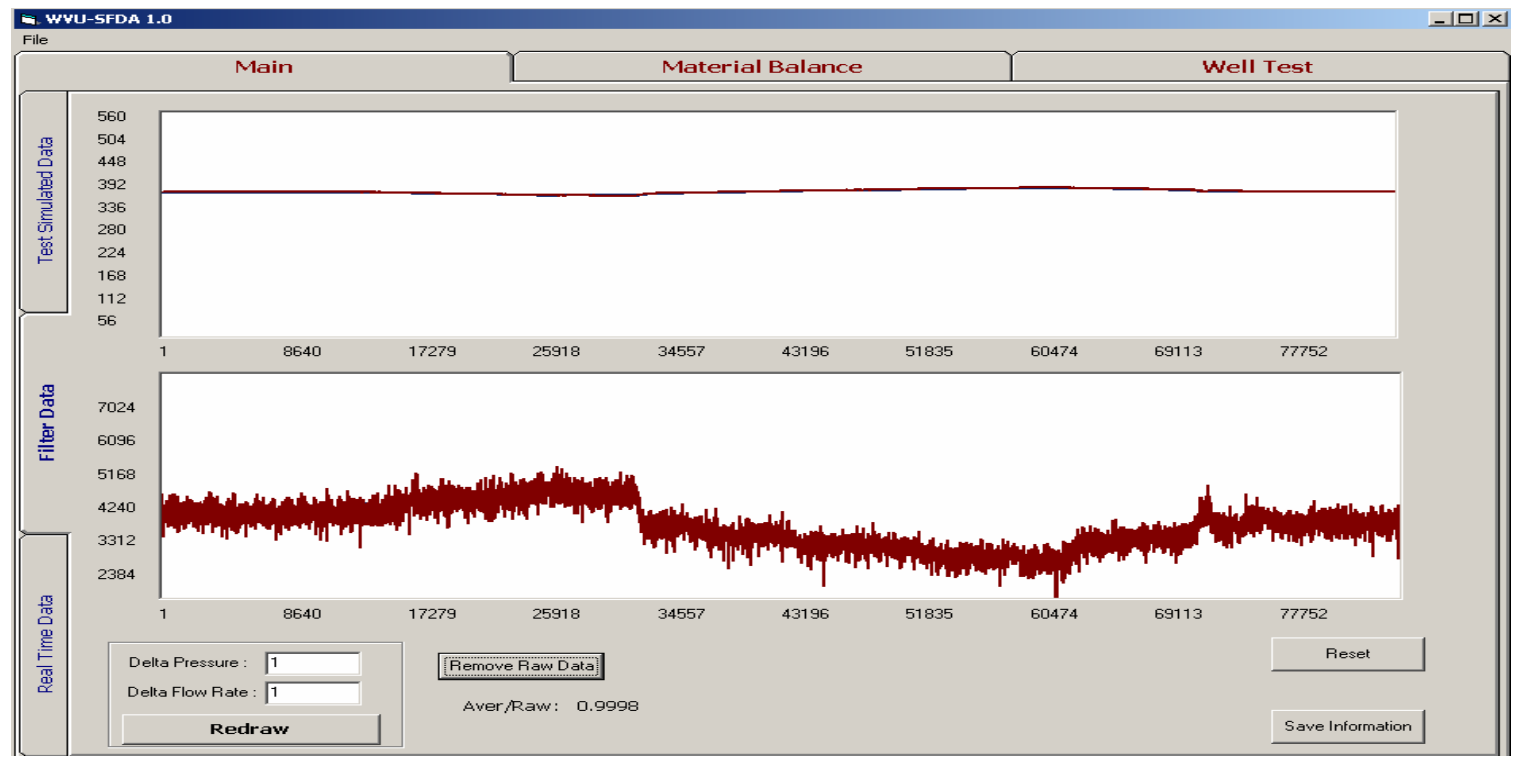

Figure 3.8: Real Time Data (Jan 31-2004)-Actual data overlapping summarized data

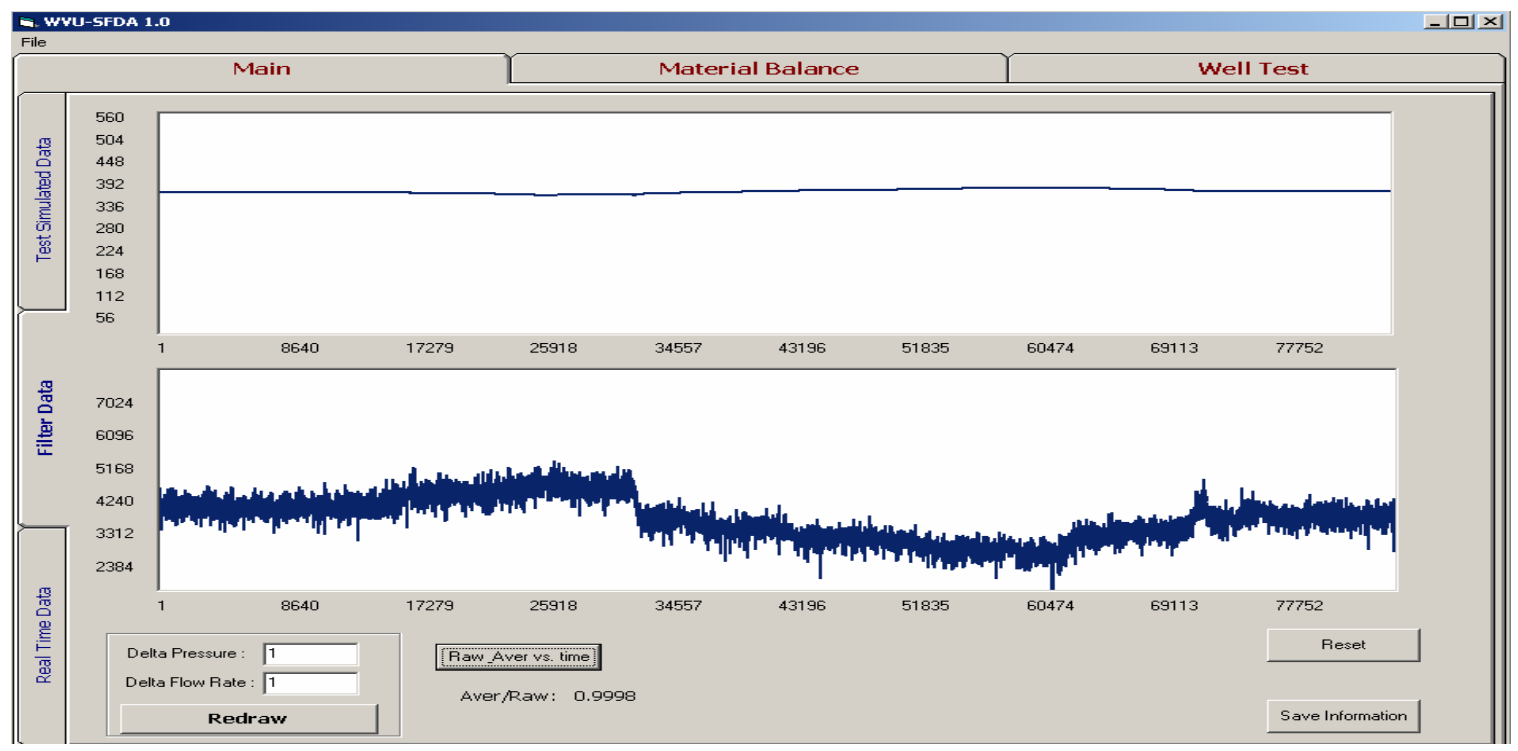

Figure 3.9: Real Time Data (Jan 31-2004)-Summarized data 
The next two figures show a case very similar to the previous example. Figure 3 shows actual and summarized data overlapping each other where the values of $\Delta \mathrm{P}$ Preset and $\Delta \mathrm{Q}$ Preset were very high. Even though the summarized data contains a few data points after using high values of $\Delta \mathrm{P}$ Preset and $\Delta \mathrm{Q}$ Preset, the summarized data (blue lines) and the actual data (red lines) are not similar as it can be observed. Figure 4 shows only the summarized data.

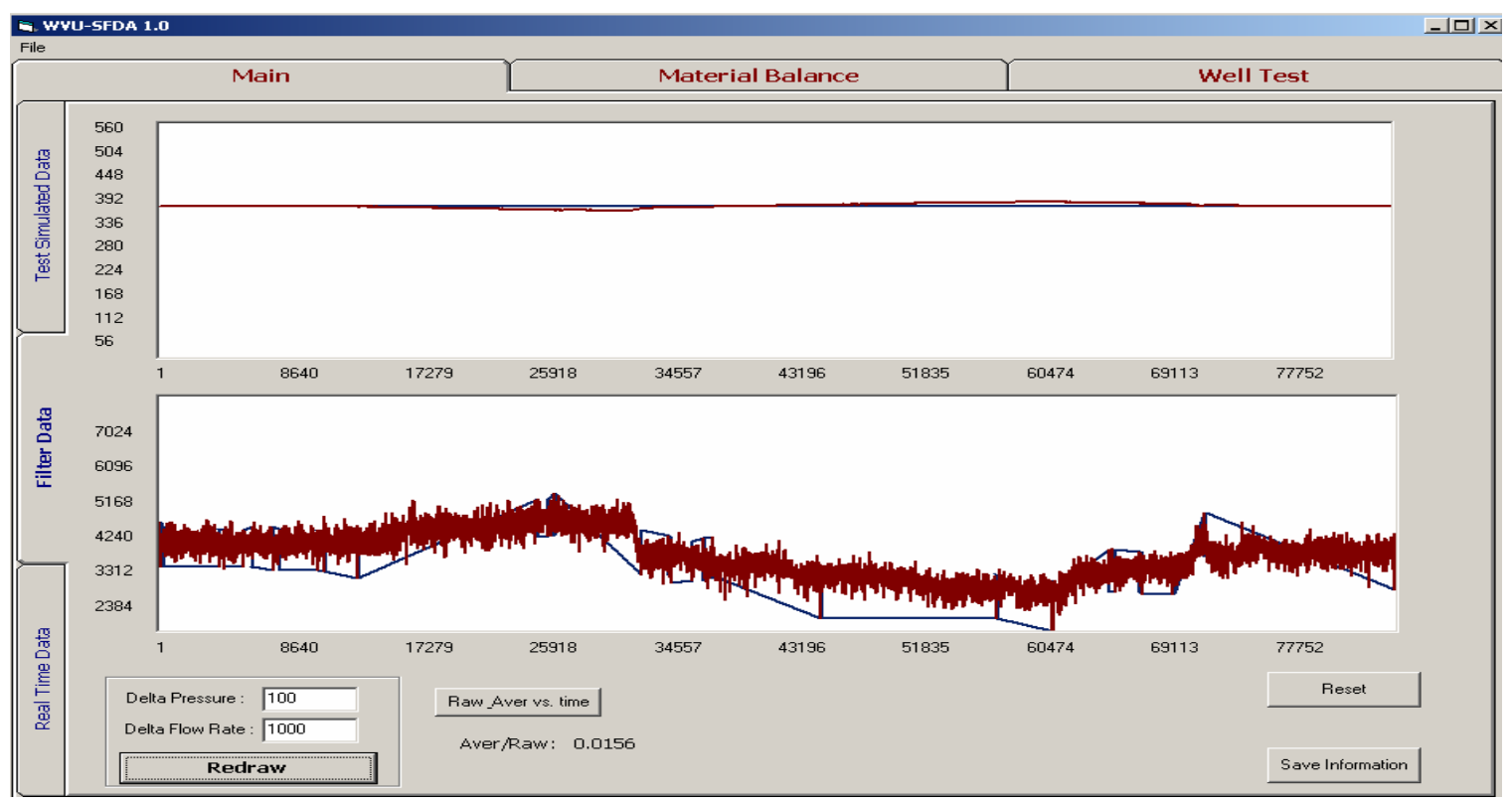

Figure 3.10: Real Time Data (Jan 31-2004)-Actual data overlapping summarized data

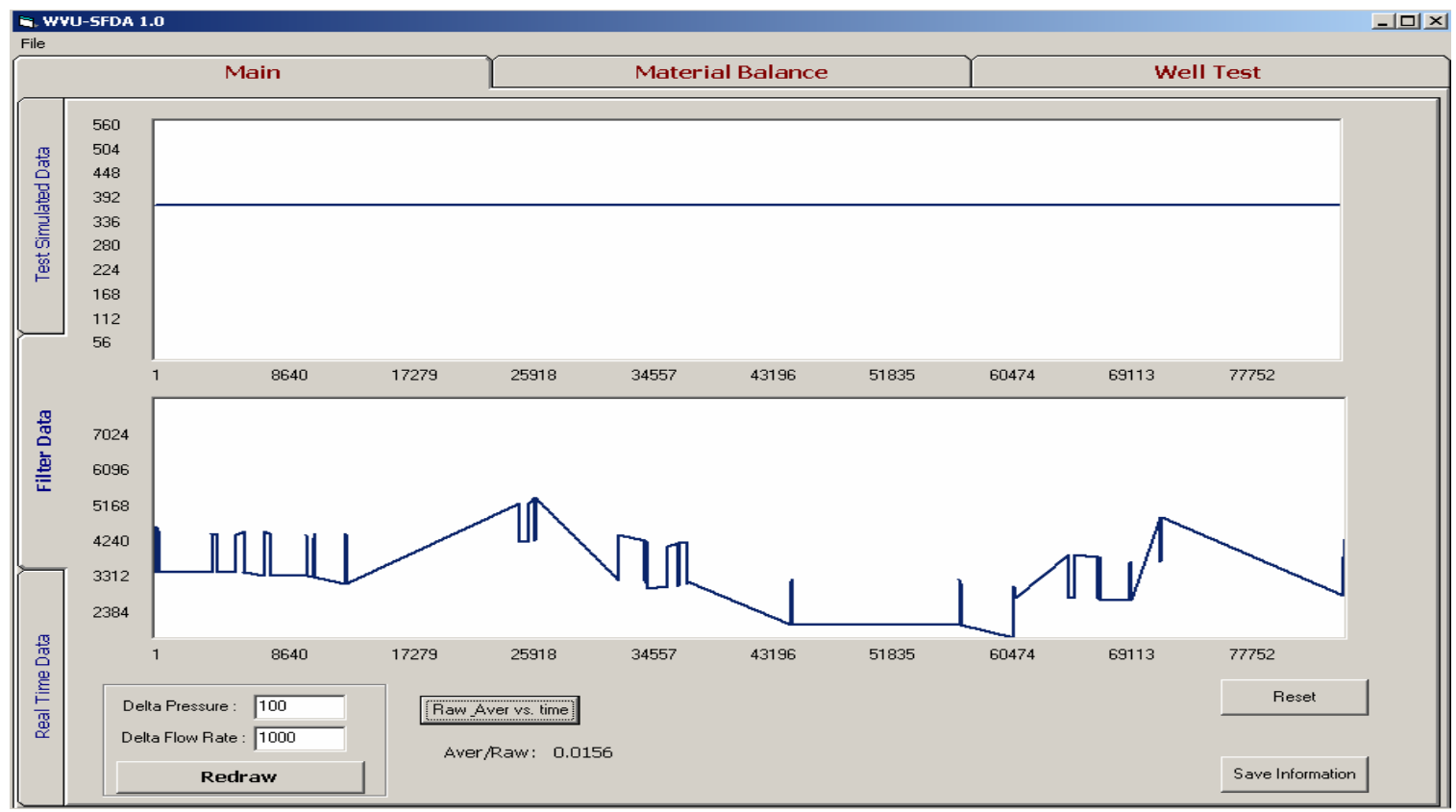

Figure 3.11: Real Time Data (Jan 31-2004)-Summarized data 


\subsubsection{Flow Rate average process}

Flow rate averaging process is based on finding $\Delta \mathrm{Q}_{\text {Preset }}$ values at different flow rate intervals. The method consists on generating random flow rate data for ten minutes between two values (e.g. $4000 \mathrm{Mcf}-5000 \mathrm{Mcf}$ ). Select a $\Delta$ QPreset and record flow rate values whenever flow rate changes $(\Delta \mathrm{Q})$ are equal or higher than the previously selected $\Delta Q_{\text {Preset. }}$ Finally, cumulative gas production for the raw and summarized data will be obtained and a relationship between those values will tell us whether the chosen $\Delta \mathrm{QPreset}$ give us summarized data that truly represent the actual data. A relationship of $95-97 \%$ will be used to determine whether the selected $\Delta Q_{\text {Preset }}$ can be used for such an interval of flow rate values. The following table and plots exemplify the methodology to average flow rate data using random data from $4000 \mathrm{Mcf}-5000 \mathrm{Mcf}$ for 10 minutes.

\begin{tabular}{|l|l|l|l|}
\hline $\begin{array}{l}\text { Time } \\
\text { Sec })\end{array}$ & $\begin{array}{l}\text { Flow Rate } \\
\text { (Mcf) }\end{array}$ & $\begin{array}{l}\text { Delta Q } \\
\text { (Mcf) }\end{array}$ & $\begin{array}{l}\text { Summarized } \\
\text { Mcf) }\end{array}$ \\
\hline 0 & 4000.0 & 0.0 & 4000.0 \\
\hline 15 & 4500.0 & 500.0 & 4500.0 \\
\hline 30 & 4300.0 & 200.0 & 4300.0 \\
\hline 45 & 4200.0 & 100.0 & 4300.0 \\
\hline 60 & 4500.0 & 200.0 & 4500.0 \\
\hline 75 & 4600.0 & 100.0 & 4500.0 \\
\hline 90 & 4500.0 & 0.0 & 4500.0 \\
\hline 105 & 4200.0 & 300.0 & 4200.0 \\
\hline 120 & 4100.0 & 100.0 & 4200.0 \\
\hline 135 & 4440.0 & 240.0 & 4440.0 \\
\hline 150 & 4800.0 & 360.0 & 4800.0 \\
\hline$"$ & & & $"$ \\
\hline & Gprd & & Gpfl \\
\hline Gprd /Gpsm & & & 0.99 \\
\hline
\end{tabular}

Table 3.8-Random Data-Flow Rate Aver. Process

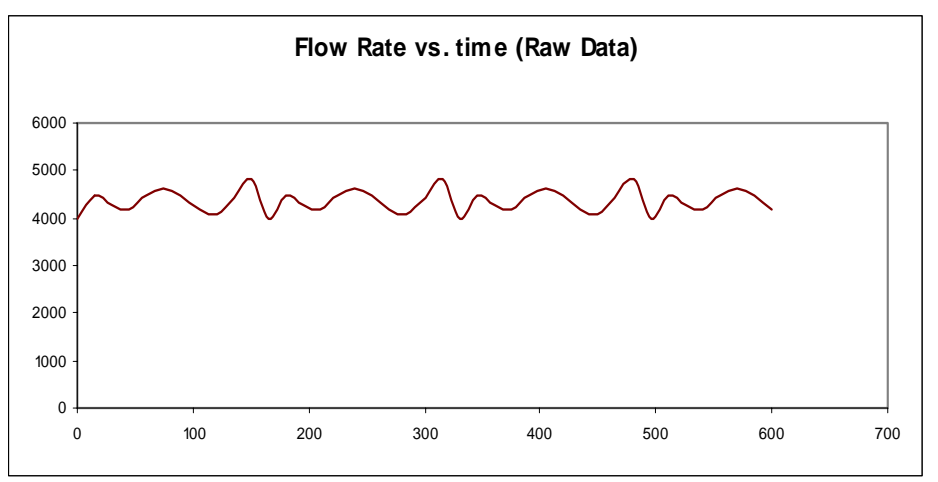

Figure 3.12: Raw Data-Random Set

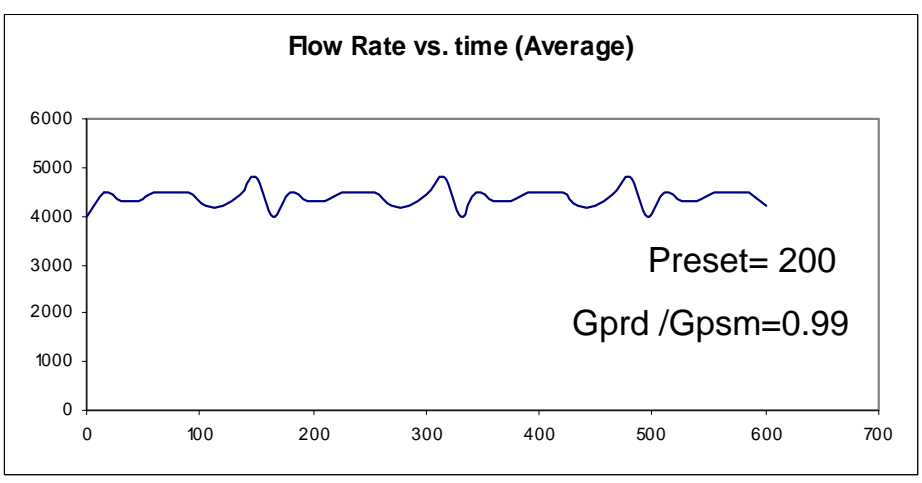

Figure 3.13: Summarized Data-Random Set-Preset $=200$ 
As it can be seen from the previous example, a $\Delta Q_{\text {Preset }}$ value of $200 \mathrm{Mcf}$ in a flow rate interval between $4000 \mathrm{Mcf}-5000 \mathrm{Mcf}$ gives us a relationship of raw data and averaged one

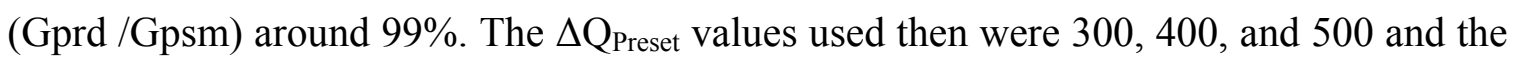
results of Gprd /Gpsm were 98\%, 97\% and 94\% respectively. As the $\Delta Q_{\text {Preset }}$ increases, the Gprd/Gpsm decreases. Since the idea was to keep a Gprd /Gpsm around 97-95\%, the proper $\Delta \mathrm{Q}_{\text {Preset }}$ value for a flow rate interval between $4000 \mathrm{Mcf}-5000 \mathrm{Mcf}$ is found to be $400 \mathrm{Mcf}$.

The plot below corresponds to the summarized data from last example using a $\Delta Q_{\text {Preset }}$ value of 400 Mcf:

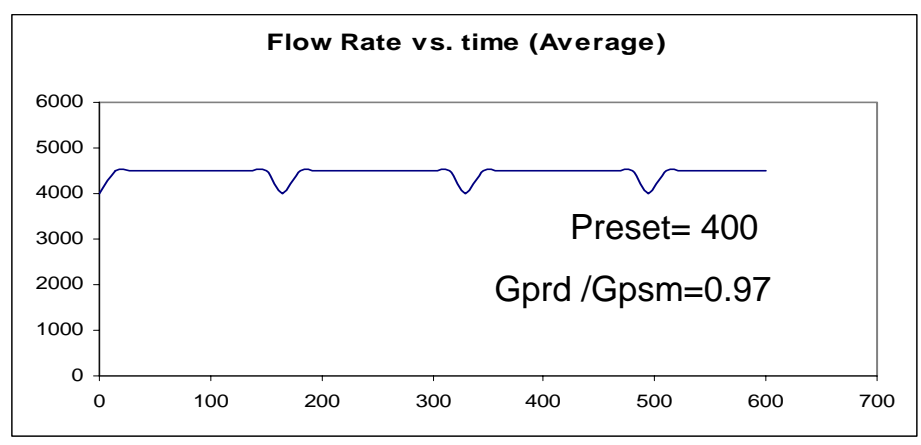

Figure 3.14: Summarized Data-Random Set-Preset $=400$

The same procedure was used for other intervals starting from 100 Mcf-200 Mcf to $1,000,000 \mathrm{Mcf}-2,000,000 \mathrm{Mcf}$ in which $\Delta \mathrm{Q}_{\text {Preset }}$ values are obtained for every interval of flow rate values.

The following tables have been generated for different $\Delta Q_{\text {Preset }}$ at several intervals in which the relationship of cumulative production between the raw data and summarized data is shown. 


\begin{tabular}{|c|c|c|c|c|c|c|c|c|}
\hline $\mathbf{Q}$ & Preset & Gpo/Gpf & $\mathbf{Q}$ & Preset & Gpo/Gpf & $\mathbf{Q}$ & Preset & Gpo/Gpf \\
\hline \multirow{3}{*}{500} & 50 & 0.98 & \multirow{3}{*}{5000} & 500 & 0.98 & \multirow{3}{*}{50000} & 5000 & 0.98 \\
\hline & 60 & 0.98 & & 600 & 0.98 & & 6000 & 0.98 \\
\hline & 70 & 0.97 & & 700 & 0.97 & & 7000 & 0.97 \\
\hline \multirow{3}{*}{600} & 60 & 0.99 & \multirow{3}{*}{6000} & 600 & 0.99 & \multirow{3}{*}{60000} & 6000 & 0.99 \\
\hline & 70 & 0.98 & & 700 & 0.98 & & 7000 & 0.98 \\
\hline & 100 & 0.95 & & 1000 & 0.95 & & 10000 & 0.95 \\
\hline \multirow{3}{*}{700} & 70 & 0.99 & \multirow{3}{*}{7000} & 700 & 0.99 & \multirow{3}{*}{70000} & 7000 & 0.99 \\
\hline & 80 & 0.98 & & 800 & 0.98 & & 8000 & 0.98 \\
\hline & 100 & 0.96 & & 1000 & 0.96 & & 10000 & 0.96 \\
\hline \multirow{3}{*}{800} & 80 & 0.98 & \multirow{3}{*}{8000} & 800 & 0.98 & \multirow{3}{*}{80000} & 8000 & 0.98 \\
\hline & 90 & 0.98 & & 900 & 0.98 & & 9000 & 0.98 \\
\hline & 100 & 0.96 & & 1000 & 0.96 & & 10000 & 0.96 \\
\hline \multirow{3}{*}{900} & 90 & 0.98 & \multirow{3}{*}{9000} & 900 & 0.98 & \multirow{3}{*}{90000} & 9000 & 0.98 \\
\hline & 100 & 0.97 & & 1000 & 0.97 & & 10000 & 0.97 \\
\hline & 110 & 0.94 & & 1100 & 0.94 & & 11000 & 0.94 \\
\hline \multirow{3}{*}{1000} & 100 & 0.99 & \multirow{3}{*}{10000} & 1000 & 0.99 & \multirow{3}{*}{100000} & 10000 & 0.99 \\
\hline & 110 & 0.98 & & 1100 & 0.98 & & 11000 & 0.98 \\
\hline & 120 & 0.95 & & 1200 & 0.95 & & 12000 & 0.95 \\
\hline \multirow{3}{*}{1100} & 100 & 0.99 & \multirow{3}{*}{11000} & 1000 & 0.99 & \multirow{3}{*}{110000} & 10000 & 0.99 \\
\hline & 110 & 0.99 & & 1100 & 0.99 & & 11000 & 0.99 \\
\hline & 120 & 0.95 & & 1200 & 0.95 & & 12000 & 0.95 \\
\hline \multirow{3}{*}{1200} & 120 & 0.99 & \multirow{3}{*}{12000} & 1200 & 0.99 & \multirow{3}{*}{120000} & 12000 & 0.99 \\
\hline & 130 & 0.99 & & 1300 & 0.99 & & 13000 & 0.99 \\
\hline & 140 & 0.97 & & 1400 & 0.97 & & 14000 & 0.97 \\
\hline \multirow{3}{*}{900} & 130 & 0.99 & \multirow{3}{*}{13000} & 1300 & 0.99 & \multirow{3}{*}{130000} & 13000 & 0.99 \\
\hline & 150 & 0.99 & & 1500 & 0.99 & & 15000 & 0.99 \\
\hline & 170 & 0.95 & & 1700 & 0.95 & & 17000 & 0.95 \\
\hline & 200 & 0.99 & & 2000 & 0.99 & & 20000 & 0.99 \\
\hline 1300 & 250 & 0.98 & 20000 & 2500 & 0.98 & 200000 & 25000 & 0.98 \\
\hline & 300 & 0.94 & & 3000 & 0.94 & & 30000 & 0.94 \\
\hline & 300 & 0.99 & & 3000 & 0.99 & & 30000 & 0.99 \\
\hline 1500 & 400 & 0.99 & 30000 & 4000 & 0.99 & 300000 & 40000 & 0.99 \\
\hline 1000 & 500 & 0.98 & 30000 & 5000 & 0.98 & 300000 & 50000 & 0.98 \\
\hline & 600 & 0.92 & & 6000 & 0.92 & & 60000 & 0.92 \\
\hline
\end{tabular}

Table 3.9 - $\Delta Q_{\text {Preset }}$ values at different flow rate values intervals

The table shown above contains several flow rate values and their respective preset values that the software will take. For instance, if the program reads a flow rate value of around 550 Mcf, SFDA will take a value of 50 as a preset. The program will discard values in which the difference between $550 \mathrm{Mcf}$ and the next value is less than its preset (50Mcf). On the other hand, if the difference between the following value and the 550 Mcf is greater than $50 \mathrm{Mcf}$, SFDA will take such a value and send it to a file containing only summarized data. 


\subsubsection{Pressure average process}

The pressure averaging process consists of finding $\Delta \mathrm{P}$ Preset values at different pressure intervals similar to the flow rate averaging process. In this case, material balance has been performed to find out a relationship of gases in place between raw and summarized data at certain $\Delta \mathrm{P}$ Preset values. For instance, if the pressure value is 350 psia and increases to $360 \mathrm{psia}$ (which lies in an interval of 300 psia- $400 \mathrm{psia}$ ), and the $\Delta \mathrm{P}$ Preset selected is 10 psia, the new averaged value would be 350 psia and the new raw value or the value not filtered would be $360 \mathrm{psia}$. After performing material balance, the results might indicate a value of 0.92 or $92 \%$ meaning that the $\Delta \mathrm{P}$ Preset selected is very high and should be decreased until the relationship of gas in place between the raw and summarized data is around $95-97 \%$.

The following table summarizes the different values of $\Delta \mathrm{P}$ Preset at different intervals of pressure values. 


\begin{tabular}{|c|c|c|c|c|c|}
\hline $\mathbf{P}$ & Preset & Grw/Gsm & $\mathbf{P}$ & Preset & Grw/Gsm \\
\hline \multirow{3}{*}{100} & 0.7 & 0.99 & \multirow{3}{*}{2000} & 20 & 0.98 \\
\hline & 0.9 & 0.98 & & 30 & 0.98 \\
\hline & 1 & 0.97 & & 40 & 0.97 \\
\hline \multirow{3}{*}{200} & 1 & 0.98 & \multirow{3}{*}{3000} & 50 & 0.98 \\
\hline & 1.5 & 0.98 & & 60 & 0.98 \\
\hline & 2 & 0.97 & & 70 & 0.97 \\
\hline \multirow{3}{*}{300} & 1 & 0.99 & \multirow{3}{*}{4000} & 80 & 0.98 \\
\hline & 2 & 0.98 & & 90 & 0.98 \\
\hline & 3 & 0.98 & & 100 & 0.97 \\
\hline \multirow{3}{*}{400} & 3 & 0.98 & \multirow{3}{*}{5000} & 120 & 0.98 \\
\hline & 4 & 0.98 & & 150 & 0.98 \\
\hline & 5 & 0.97 & & 200 & 0.97 \\
\hline \multirow{3}{*}{500} & 5 & 0.98 & \multirow{3}{*}{6000} & 250 & 0.98 \\
\hline & 6 & 0.98 & & 280 & 0.98 \\
\hline & 7 & 0.97 & & 300 & 0.97 \\
\hline \multirow{3}{*}{600} & 6 & 0.98 & \multirow{3}{*}{7000} & 310 & 0.98 \\
\hline & 7 & 0.98 & & 320 & 0.98 \\
\hline & 8 & 0.97 & & 350 & 0.97 \\
\hline \multirow{3}{*}{700} & 8 & 0.98 & \multirow{3}{*}{8000} & 400 & 0.98 \\
\hline & 9 & 0.98 & & 430 & 0.98 \\
\hline & 10 & 0.97 & & 450 & 0.97 \\
\hline \multirow{3}{*}{800} & 8 & 0.98 & \multirow{3}{*}{9000} & 500 & 0.98 \\
\hline & 9 & 0.98 & & 530 & 0.98 \\
\hline & 10 & 0.97 & & 550 & 0.97 \\
\hline \multirow{3}{*}{900} & 12 & 0.98 & \multirow{3}{*}{10000} & 600 & 0.98 \\
\hline & 13 & 0.98 & & 630 & 0.98 \\
\hline & 14 & 0.97 & & 650 & 0.97 \\
\hline \multirow{3}{*}{1000} & 10 & 0.98 & \multirow{3}{*}{15000} & 1000 & 0.98 \\
\hline & 15 & 0.98 & & 1100 & 0.98 \\
\hline & 20 & 0.97 & & 1200 & 0.97 \\
\hline \multirow{3}{*}{1500} & 15 & 0.98 & \multirow{3}{*}{20000} & 1500 & 0.98 \\
\hline & 20 & 0.98 & & 1700 & 0.98 \\
\hline & 30 & 0.97 & & 2000 & 0.97 \\
\hline
\end{tabular}

Table $3.10-\Delta \mathrm{P}_{\text {Preset }}$ values at different pressure values intervals 


\subsubsection{Summarized Data Selection Process}

Once $\Delta \mathrm{P}_{\text {Preset }} \& \Delta \mathrm{Q}_{\text {Preset }}$ values have been selected for several intervals of pressure and flow rate respectively, the process of recording and discarding such parameters simultaneously takes place. In such a process, the changes in pressure and flow rate $(\Delta \mathrm{P}$ $\& \Delta \mathrm{Q})$ are compared to their respective $\Delta \mathrm{P}_{\text {Preset }} \& \Delta \mathrm{Q}_{\text {Preset }}$ assigned values at the same time. Whenever the changes in pressure and flow rate are greater than the preset values, the summarized data will record such values. If only one change in parameter is greater than its preset value, the summarized data will not record such values.

Therefore, in order to record new values for the set of summarized data both conditions (the change in pressure must be greater than its preset value and the change in flow rate also has to be greater than its preset value) must be fulfilled. The following flow chart best summarizes this approach:

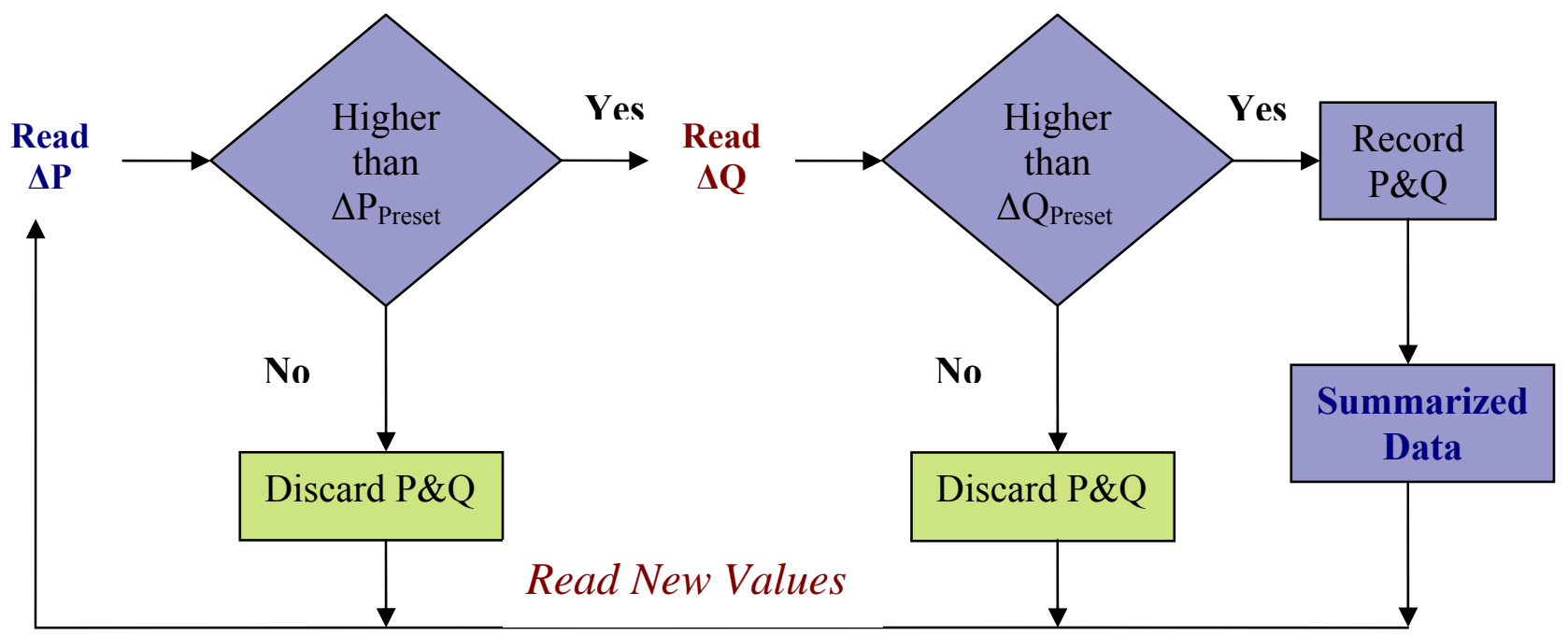

Figure 3.15: Summarized Data Selection Process 


\subsection{Pressure, Flow Rate, and Gas in Place Profiles Using Summarized Data}

Once the averaged data is obtained, the program has the option of showing pressure, flow rate, and gas in place versus time for every month. The user will have the option of detecting any abnormality that occurred during a determined month from the pressure, flow rate, and gas in place profile. In addition, on the upper part of every graph, the differences of changes in their parameters will be shown.

Gas in place is a value that should remain constant at all times. Monitoring such a parameter will allow detecting whether the gas in place decreases or remains constant. The engineers might interpret a decrease in gas in place as the existence of a leak. In such a case, the operators can be notified and the appropriate actions can be taken right away. The result section (Chapter 4) will show several cases where the gas in place has been calculated and plotted for several months.

\subsubsection{Pseudo Pressure Profile}

SFDA will have the option of showing Pseudo Pressure $(\mathrm{m}(\mathrm{p}))$ versus time. The program will allow the user to select a temperature and a specific gravity that will be used for the calculation of pseudo pressure values. Pseudo Pressure values can be used later on for well testing analysis. Once $\mathrm{m}(\mathrm{p})$ and pressure values are obtained, the user can use such values for better pressure transient analysis interpretation.

Once the temperature and specific gravity has been selected, the software will develop a table with certain gas properties values such as viscosity and $\mathrm{P} / \mathrm{Z}$-values. It will calculate pseudo pressure values at different pressure values with a $\Delta \mathrm{P}$ of $10 \mathrm{psia}$ by numerical integration. The range of $\mathrm{m}(\mathrm{p})$ values at different pressures will be from 10 psia to 10,000 psia. See example below: 


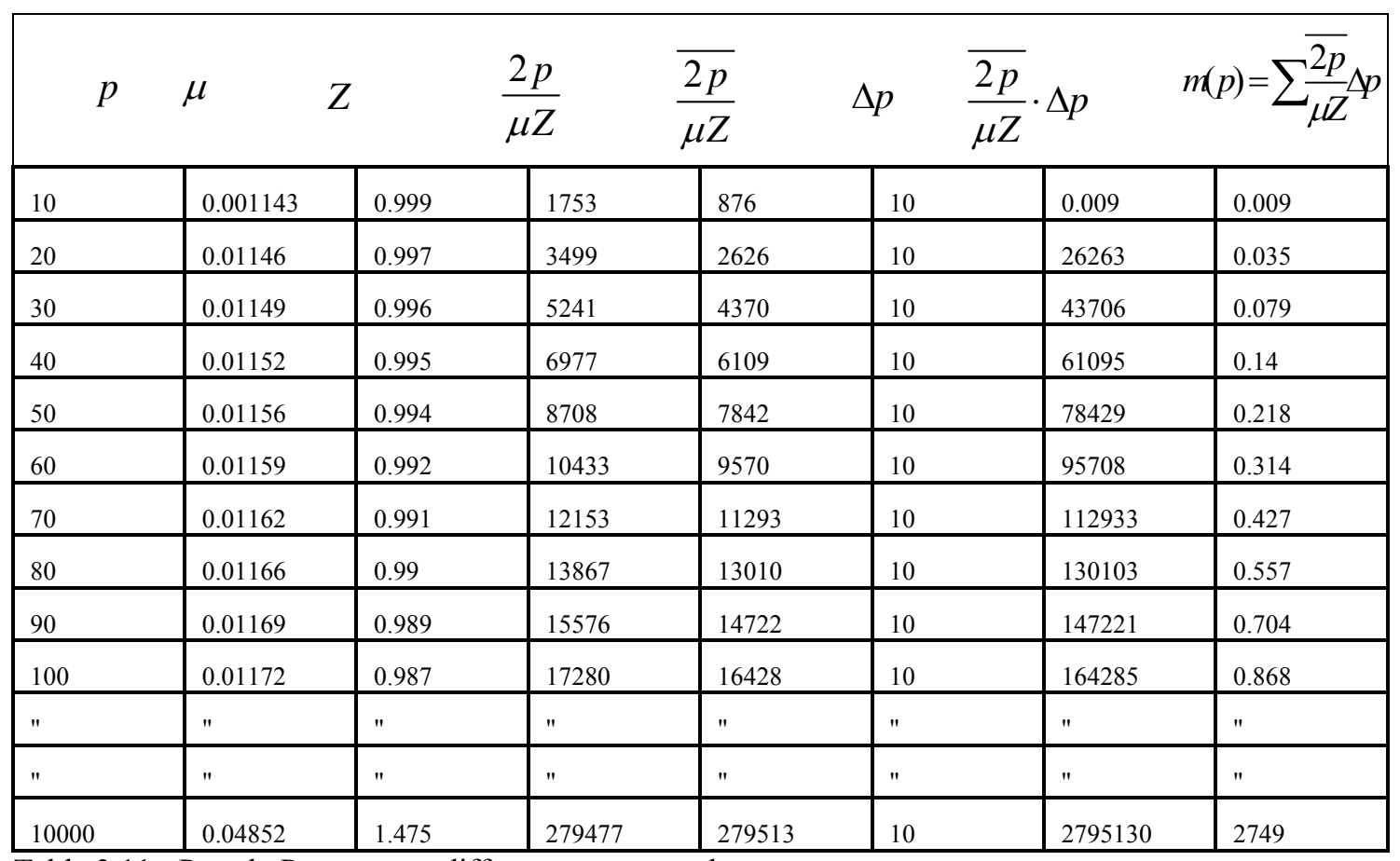

Table 3.11 - Pseudo Pressures at different pressure values

SFDA will take every value of pressure from the pressure profile and then it will go over the table to detect in which two values the actual pressure lies. Finally, it will interpolate to obtain the corresponding pseudo pressure values. The program will have the option of showing such a pseudo pressure value in function of time.

\subsubsection{Pressure Square versus Time}

SFDA will have the option of converting the pressure values from the pressure profile data to pressure square values. Such a case is used in most pressure drawdown testing in which a semi-log plot of pressure square versus time is used to calculate the slope that will then be used to obtain permeability and skin factor. Having pressure square data as well as pseudo pressure information will allow the user to perform well testing analysis in a most accurate manner. 


\subsection{Decline Curve Analysis}

SFDA will have the option of performing decline curve analysis to predict future gas production. The user will have the option of downloading the monthly production summarized data that the software can provide and choose the appropriate parameters such as b, Qi, and Di to draw the decline curve. Once the decline is drawn, future prediction about gas production for a period of a year can be made. The date at which the initial flow rate is selected will also be saved for future analysis.

Two types of decline analysis can be used by the user: production flow fate versus time, and production flow rate versus cumulative production. The first one can be used for new wells to calculate the expected production profile over a certain period of time. On the other hand, the flow rate-cumulative production plot can be used to calculate expected production profiles for production wells whose production history is available. 


\section{Chapter 4}

\section{Case Study-Results}

\subsection{Real Time Data-Columbia Gas Transmission Corporation}

Real Time Data from 20 different wells provided by Columbia Gas Transmission Corporation has been analyzed and tested using SFDA where the well distribution can be seen below. For our particular case, well 12336 was analyzed and tested for different days and months.

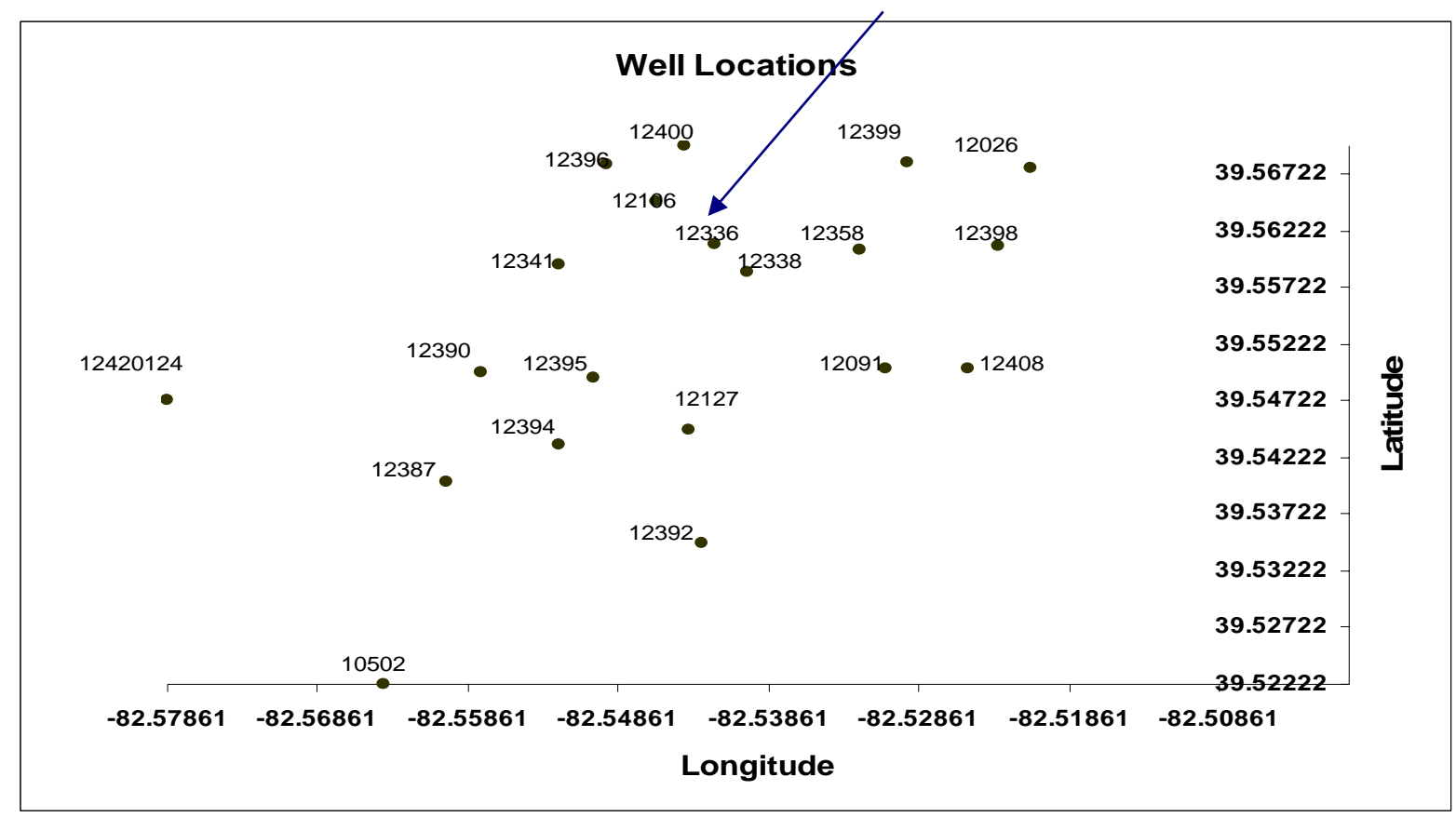

Figure 4.1-Well Locations-Data Provided 


\subsection{Pressure Transient Analysis}

Columbia Gas Transmission Corporation provided us with some well test data at a very small interval of time. As explained before in the methodology section, SFDA is capable of detecting any pressure transient period, saving well testing data, and preparing such information for the user to analyze. The following examples show some of the well testing data detected by the program and its approach to calculate slope, permeability, and skin factor.

Whenever a transient period is detected by the software, it will be notified in the status section. Reading status means that SFDA is in the process of saving well testing data in a folder and static status indicates that the program has already detected a pressure transient period and the information is saved and ready for well test analysis.

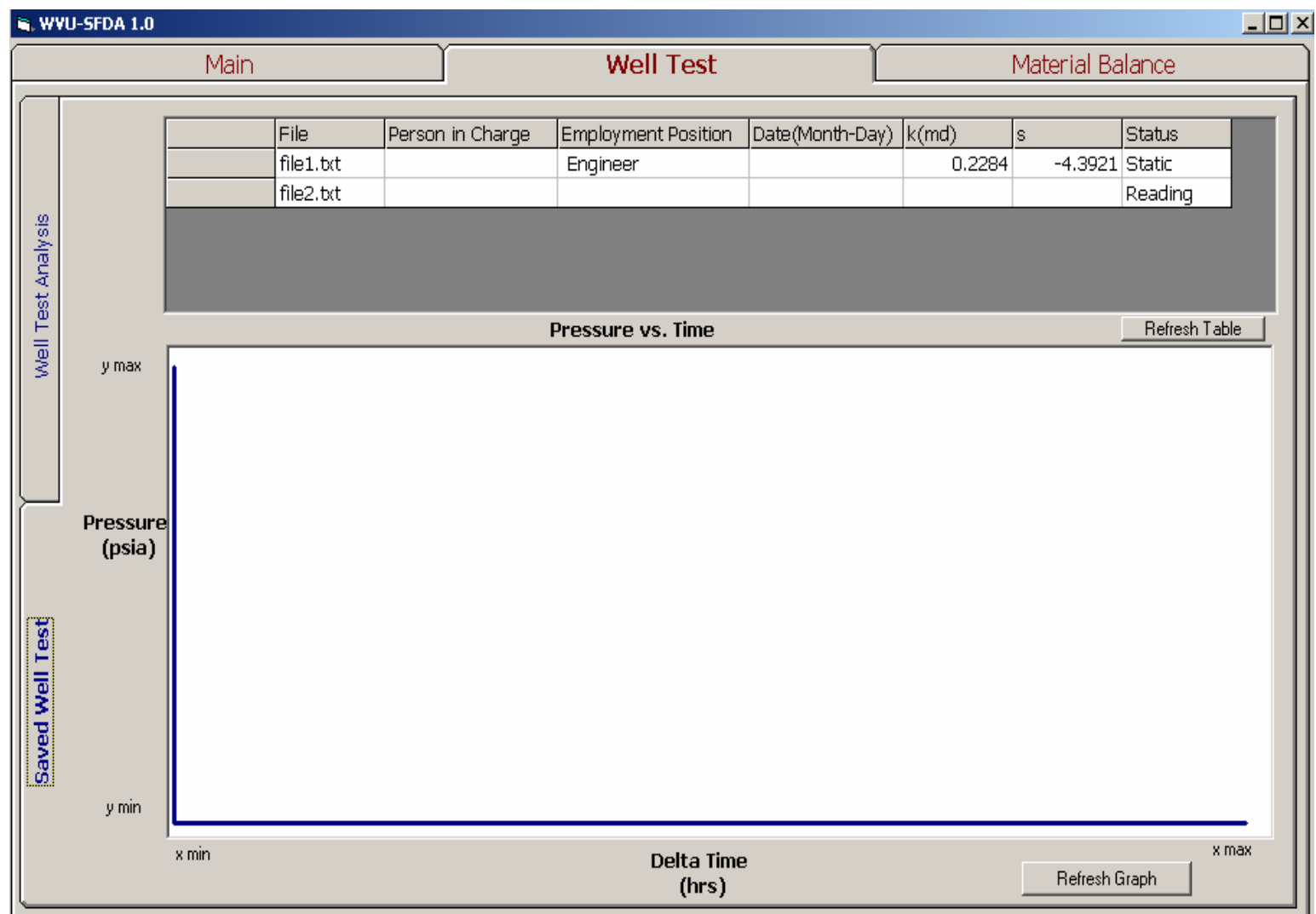

Figure 4.2-Pressure Transient Period detection 
Once the user clicks on the file number, a plot of pressure versus time will appear and a new form asking for user information will pop up.

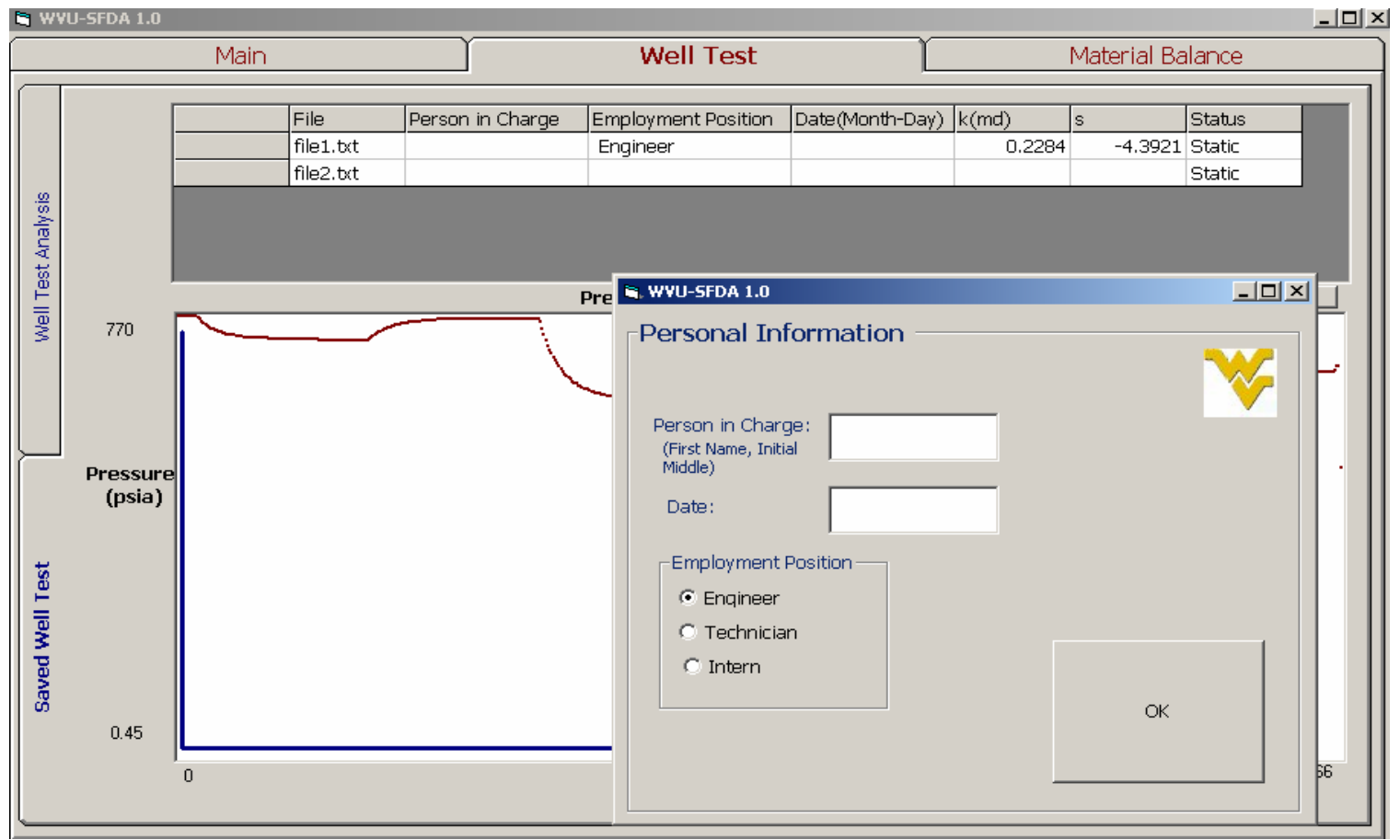

Figure 4.3-Well Test Information for Pressure vs. time and Personal Information window

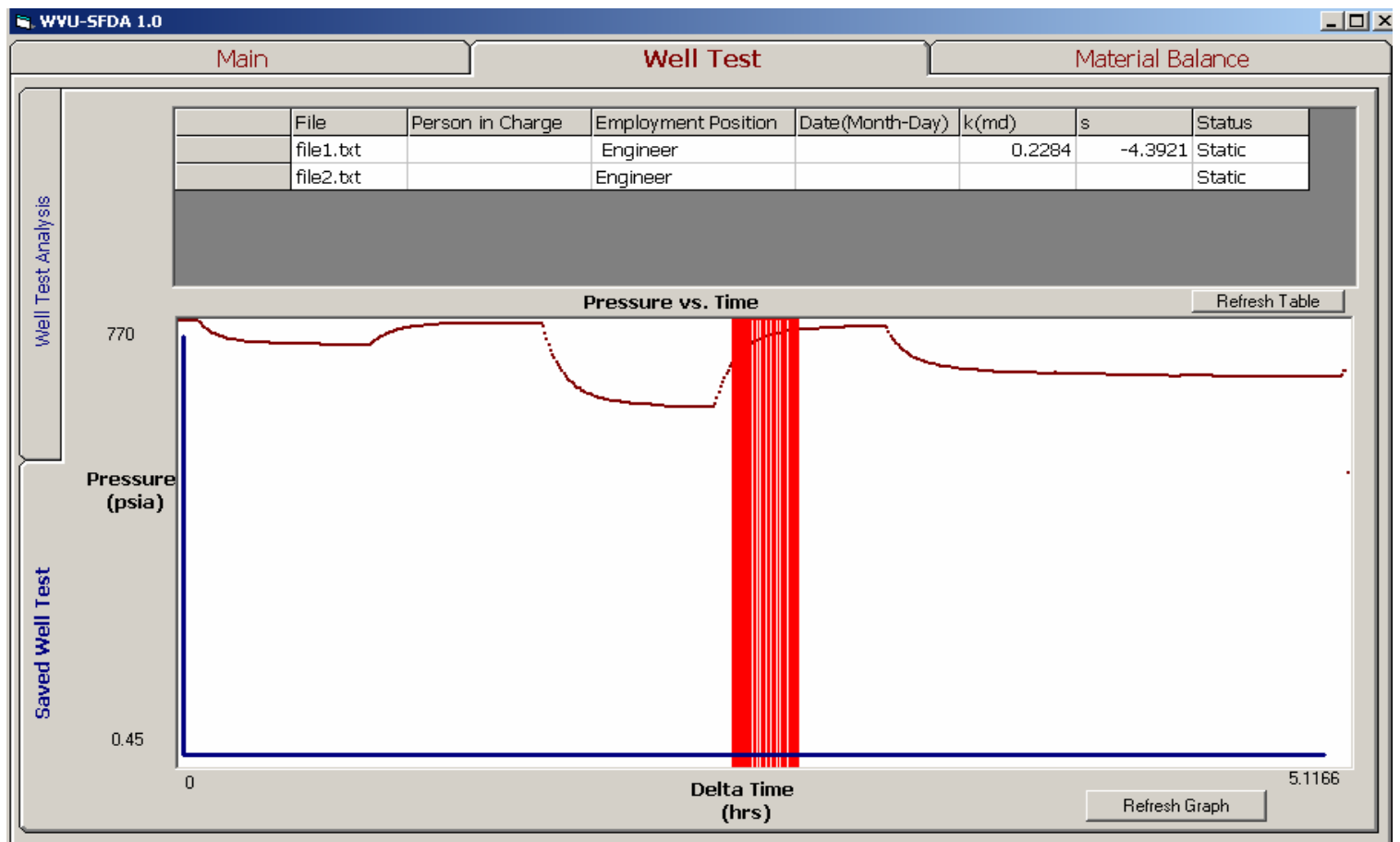

Figure 4.4-Area selection for Horner Plot 
A new window showing the Horner plot will appear in which the user will draw the slope of a line to obtain permeability and skin factor.

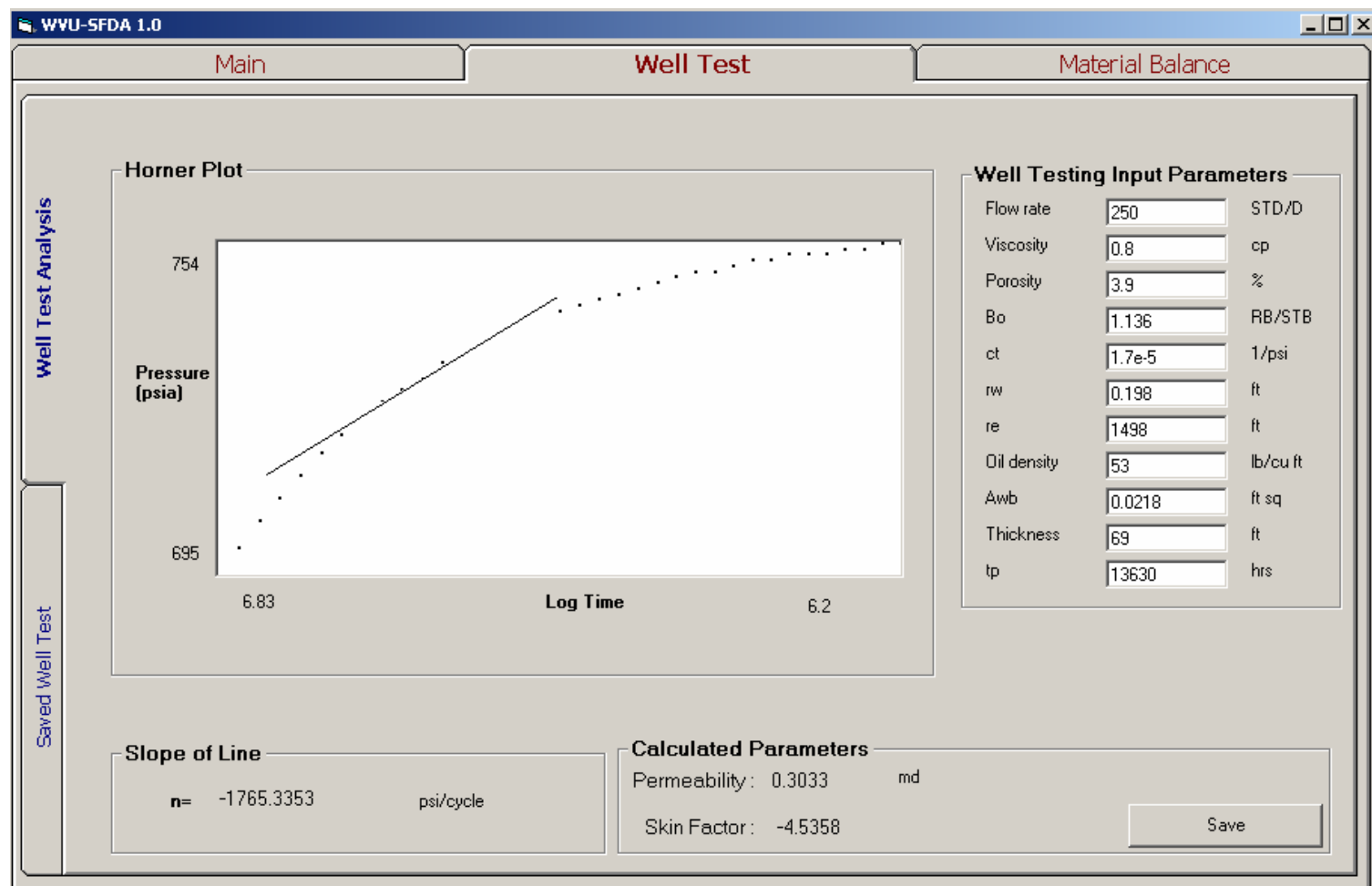

Figure 4.5-Horner Plot and Line Drawn to obtain permeability and skin factor

The user will have the option of saving values of permeability and skin factor for different build-up periods.

\subsection{Summarized Data Results Analysis}

The next figures will show the results of SFDA after reading data in real time and summarizing it to obtain a lesser amount of data and a plot very similar to the actual one. The curves overlap where the red one corresponds to the actual values and the blue one to the averaged data. 


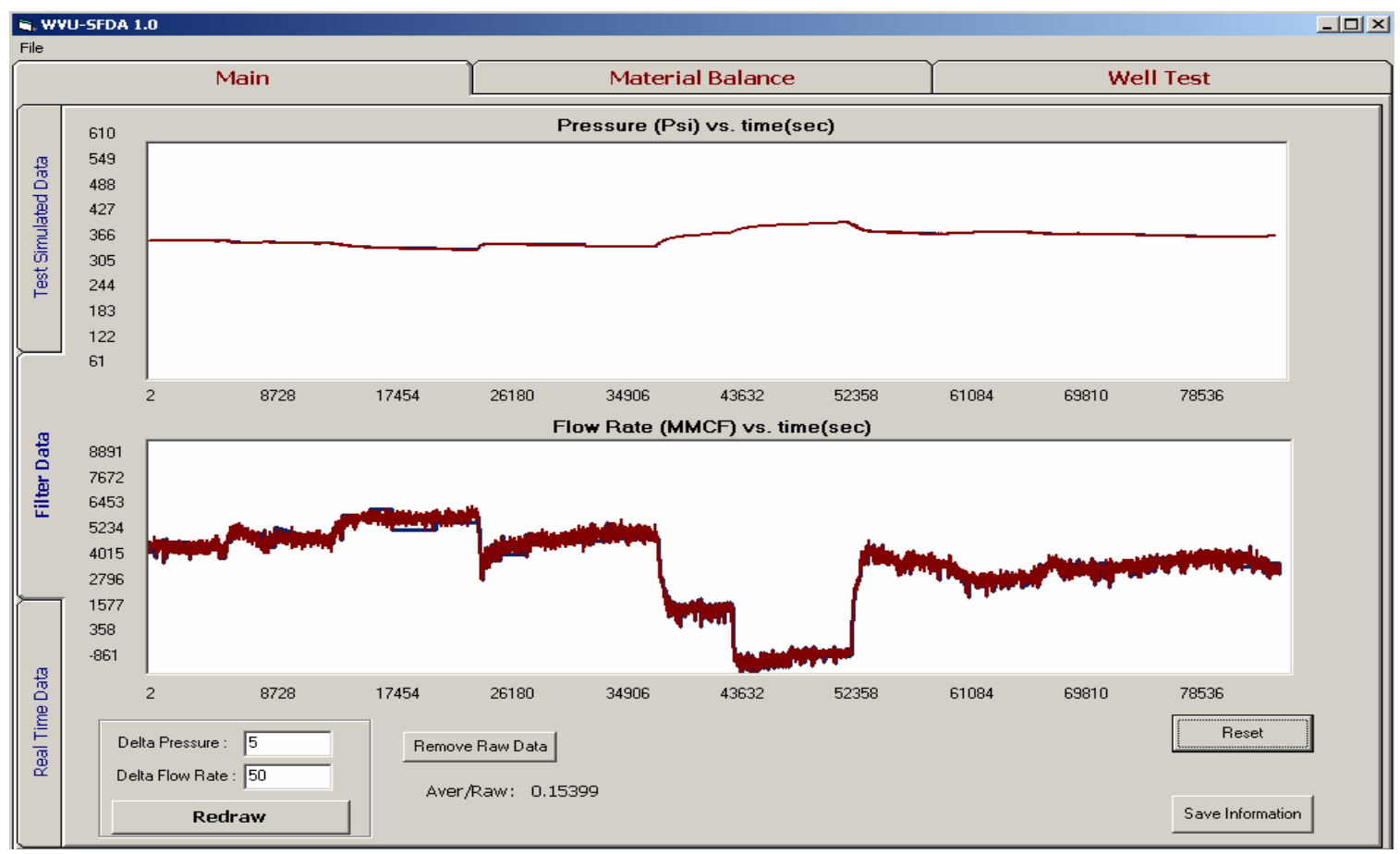

Figure 4.6-Pressure and Flow Rate- Actual and Summarized Data (Jan 27, 05)

Even though the blue curve seems not to be present, it is actually below the red one. The following figure will show only the summarized data. (The summarized data is using $15 \%$ of the actual data and discarding $85 \%$ ):

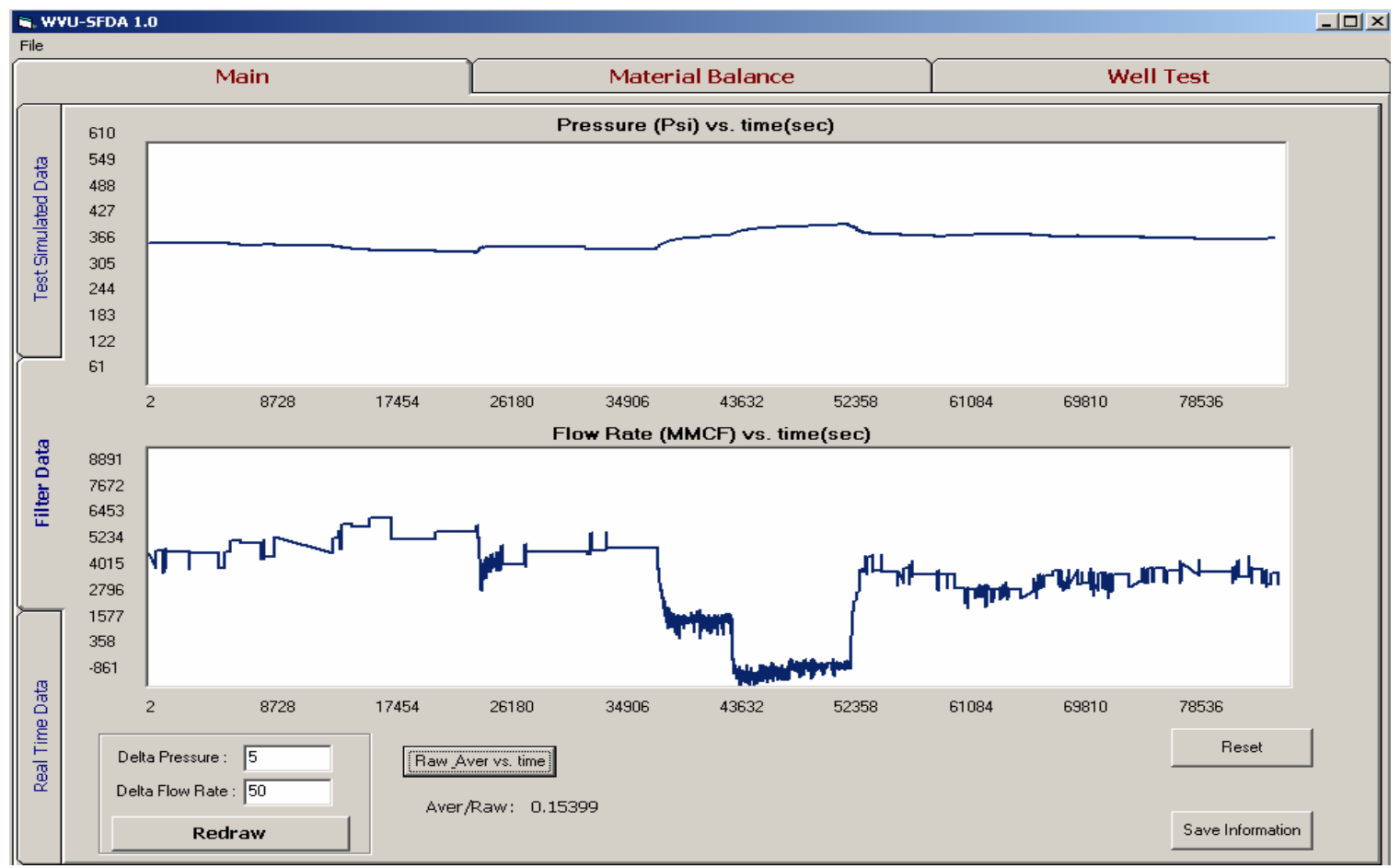

Figure 4.7-Pressure and Flow Rate -Summarized Data (Jan 27, 05) 
The next figures correspond to Real Time Data from Jan 14, 2005:

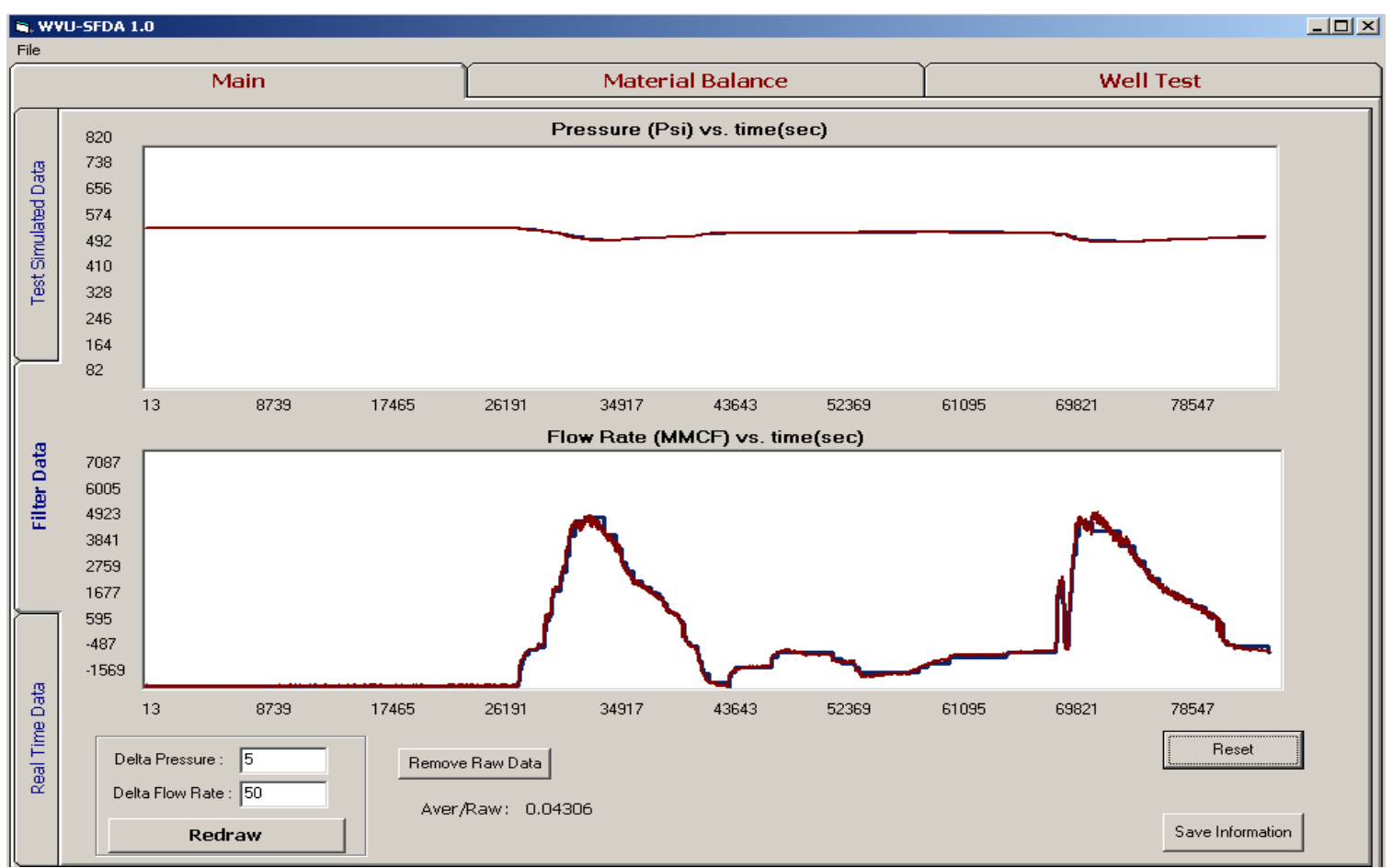

Figure 4.8-Pressure and Flow Rate -Actual and Summarized Data (Jan 14, 05)

For this case the summarized data is using about $4 \%$ of the total data $(96 \%$ has been discarded):

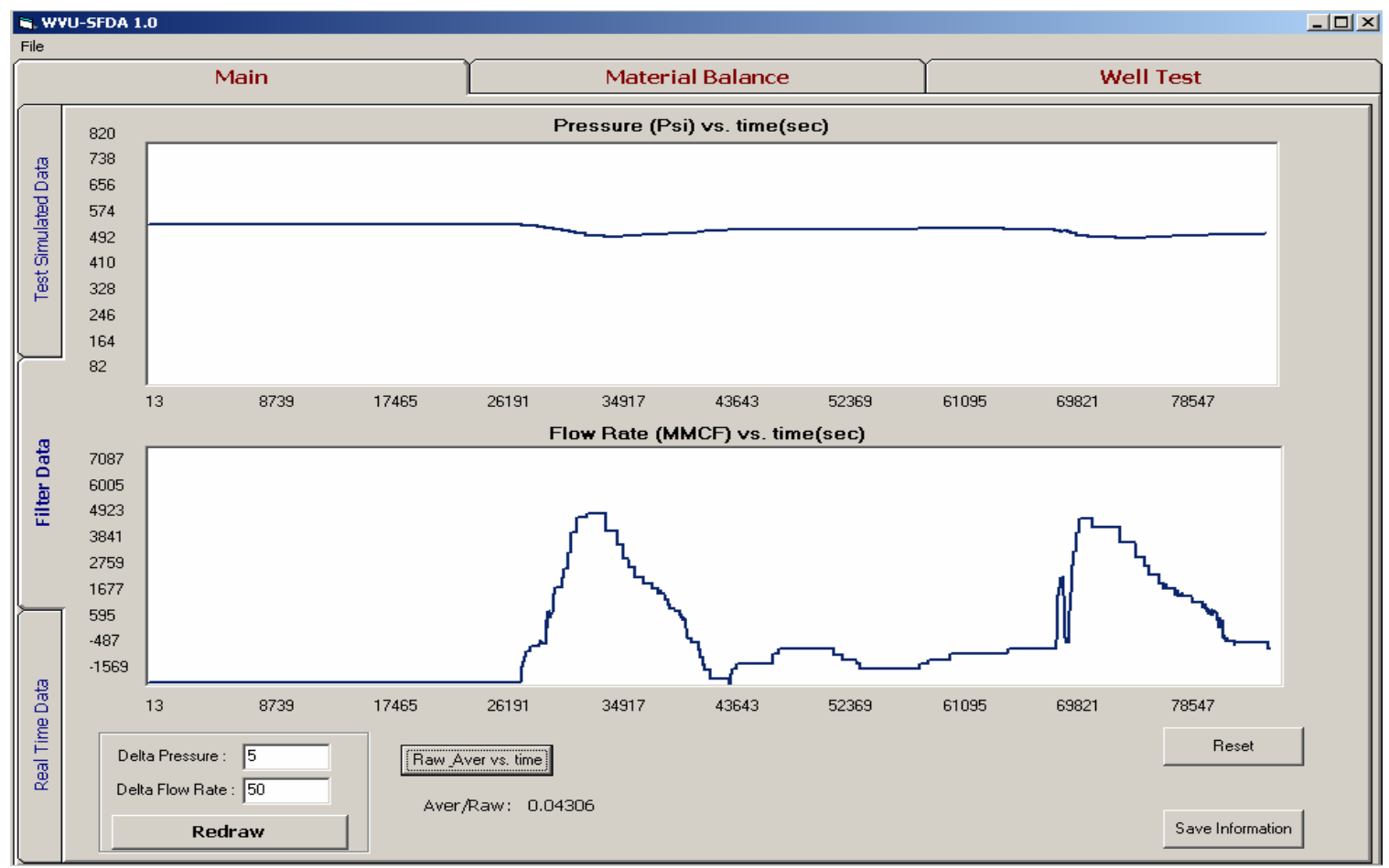

Figure 4.9-Pressure and Flow Rate- Summarized Data (Jan 14, 05) 
The next figures correspond to Real Time Data from Feb 20, 2005:

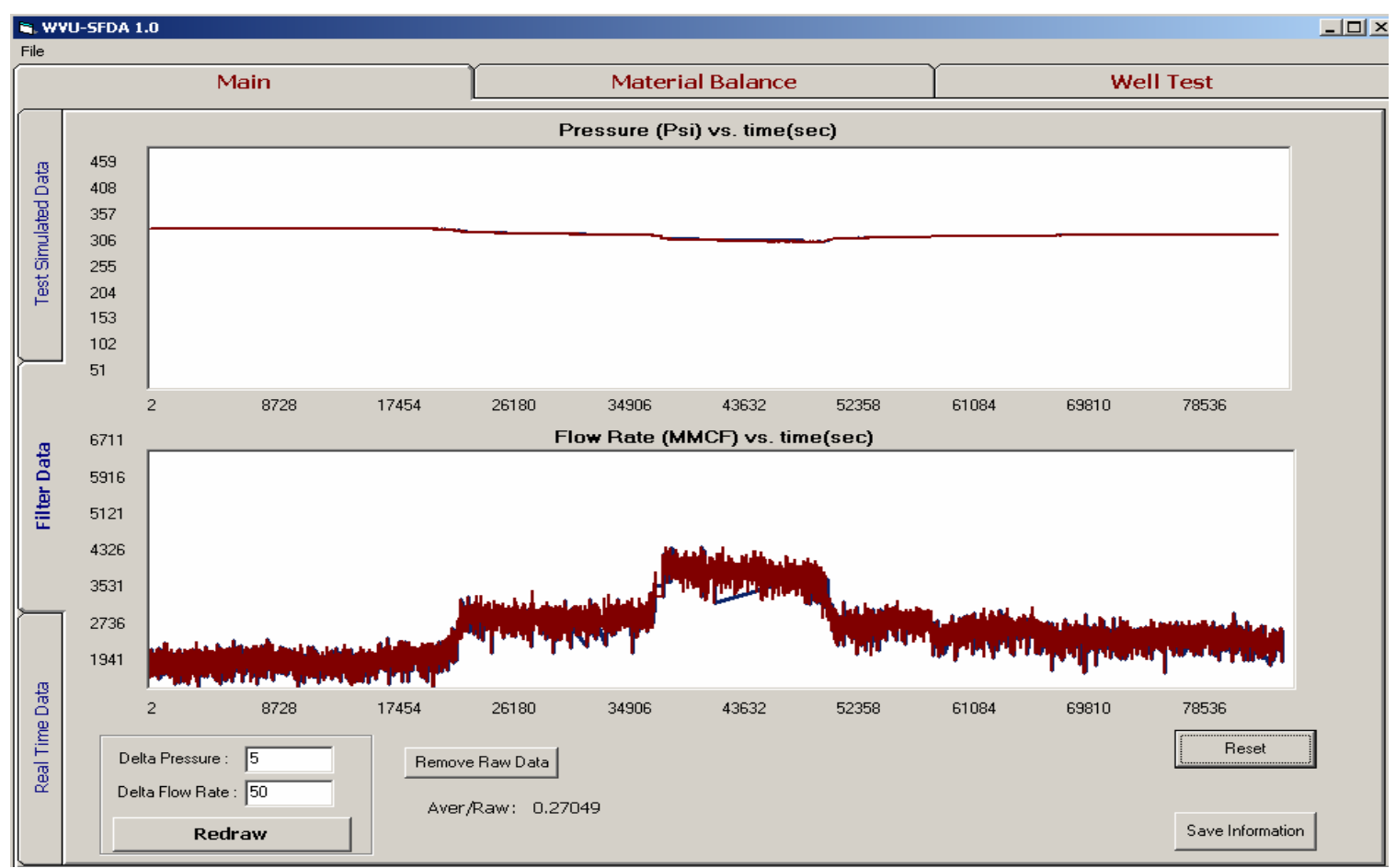

Figure 4.10-Pressure and Flow Rate- Actual and Summarized Data (Feb 20, 05)

For this case, the summarized data is using about $27 \%$ of the total data $(73 \%$ has been discarded):

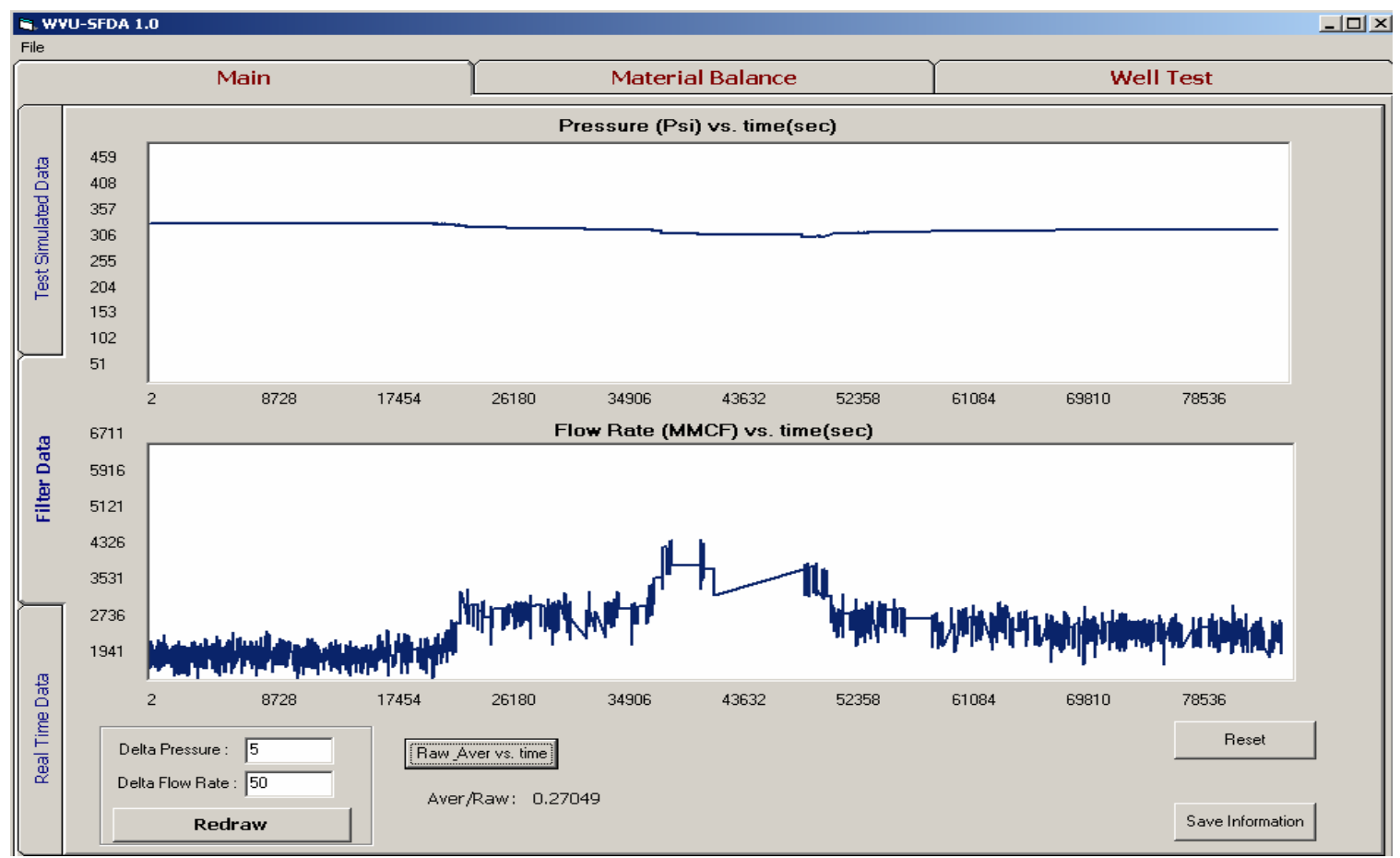

Figure 4.11-Pressure and Flow Rate -Summarized Data (Feb 20, 05) 
The next figure corresponds to Real Time Data from Feb 23, 2005:

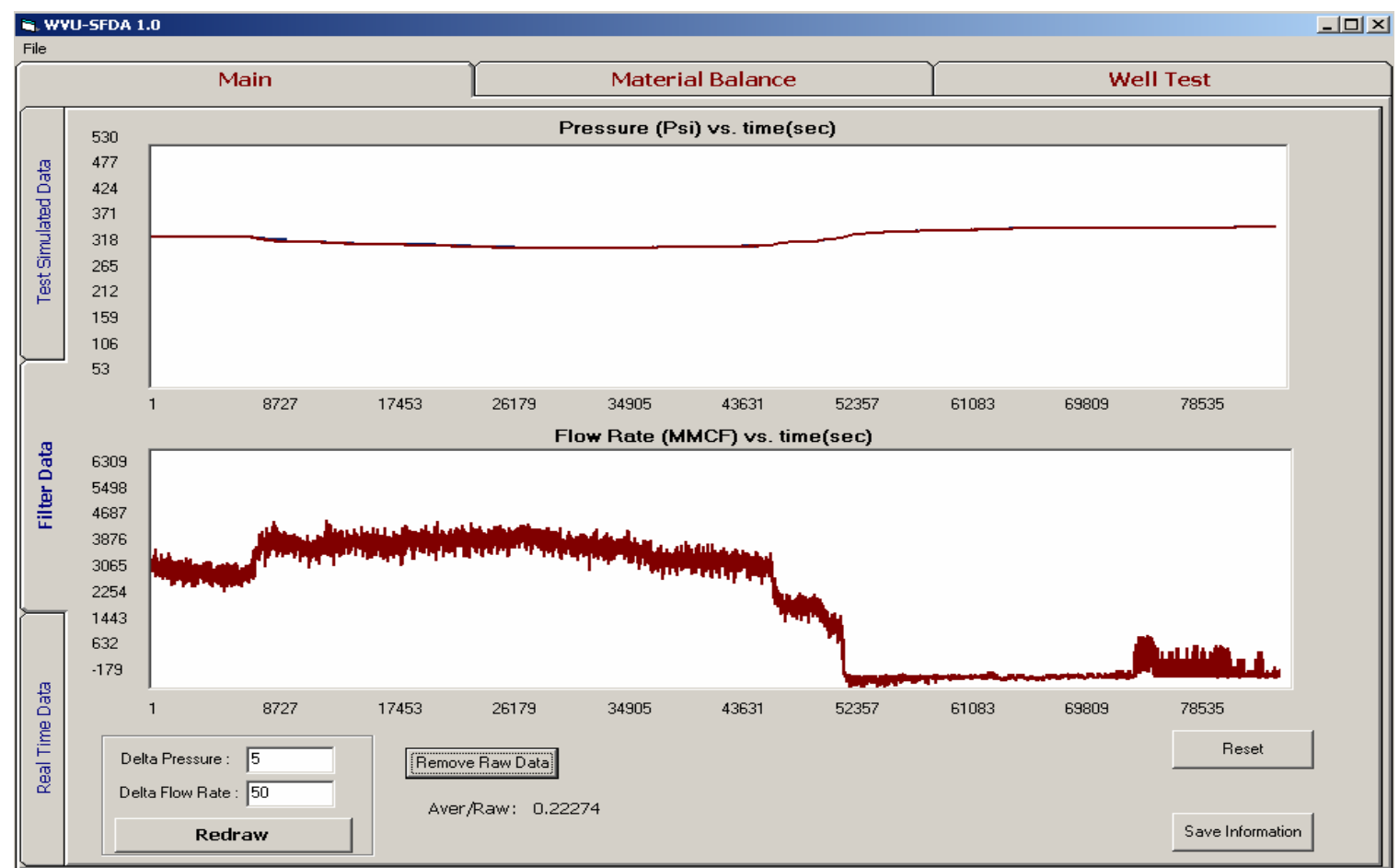

Figure 4.12-Pressure and Flow Rate- Actual and Summarized Data (Feb 23, 05)

For this case, the summarized data is using about $22 \%$ of the total data $(78 \%$ has been discarded):

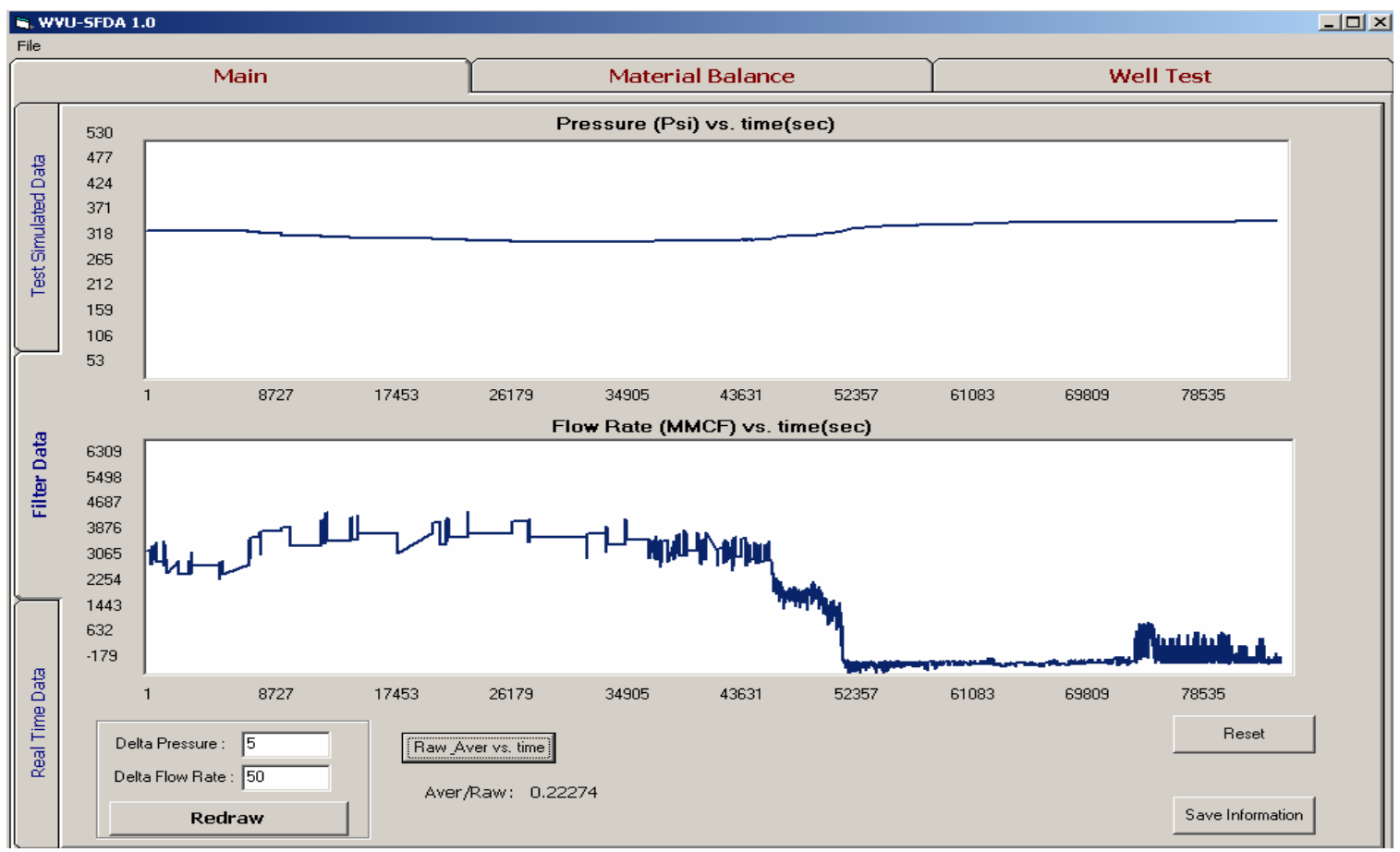

Figure 4.13-Pressure and Flow Rate -Summarized Data (Feb 23, 05) 
The next figures correspond to Real Time Data from March 14, 2005:

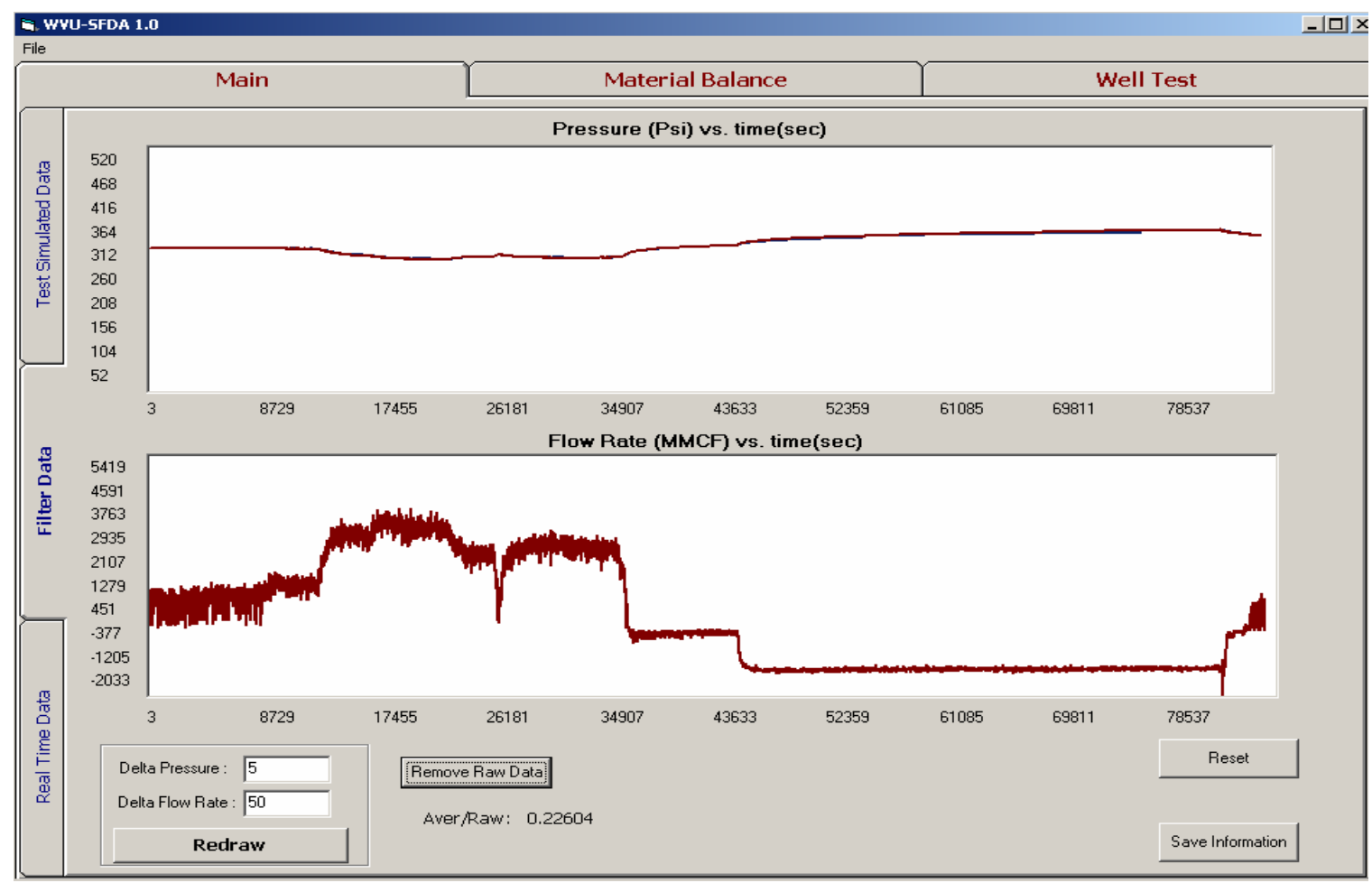

Figure 4.14-Pressure and Flow Rate- Actual and Summarized Data (March 14, 05)

For this case, the summarized data is using about $22 \%$ of the total data $(78 \%$ has been discarded):

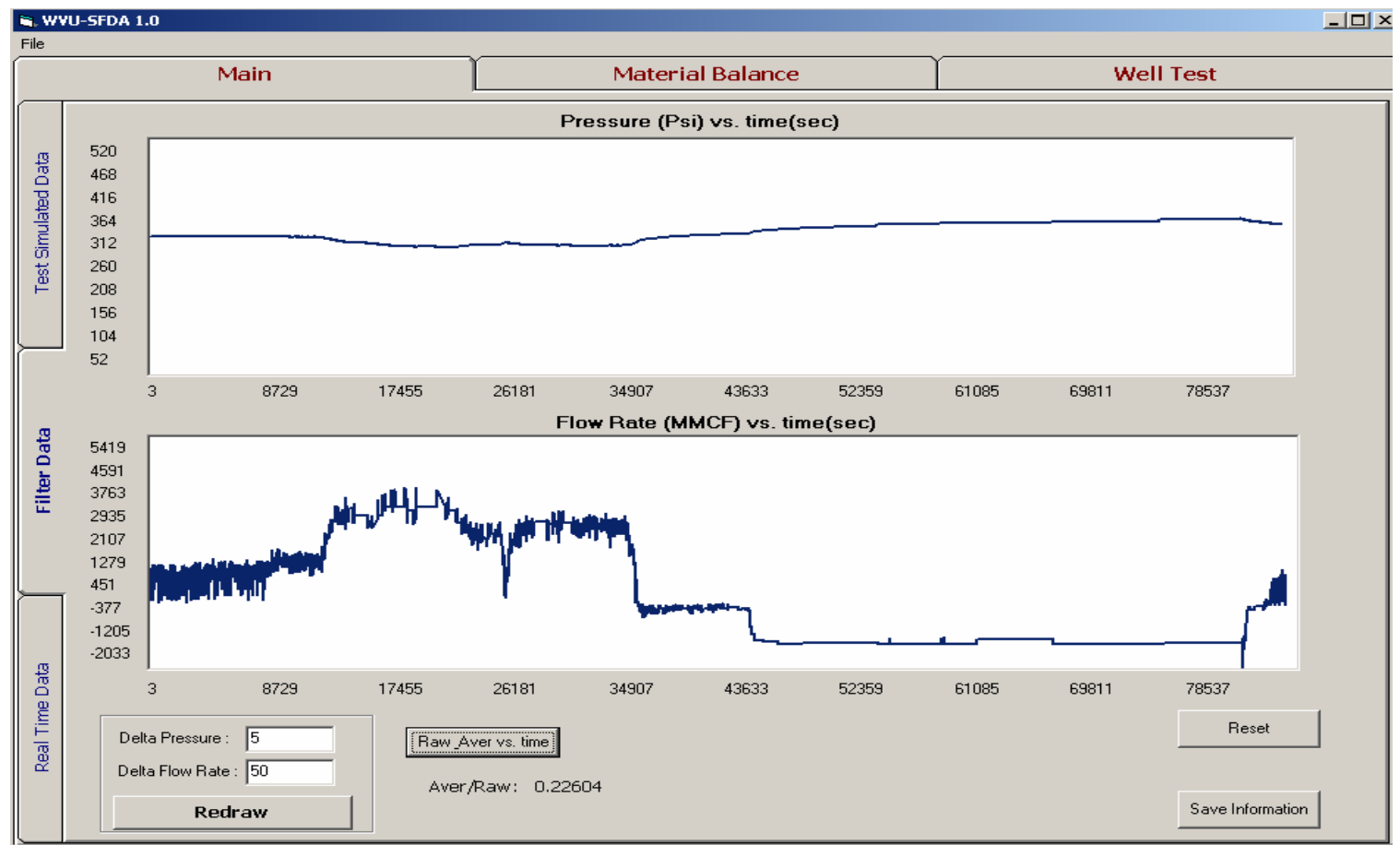

Figure 4.15-Pressure and Flow Rate -Summarized Data (March 14, 05) 
After SFDA has read data from a period of a day, summarized it, and sent such files to a folder that contains the averaged data, the user will be capable of downloading such information and checking whether the criteria used by the software to aggregate the data is valid. In others words, the user will be able to evaluate how the summarized data differs from the actual data. If the user considers that the summarized data is very different from the actual one, this person can change the preset values and redraw the summarized data and the actual values until both curves match.

After running data from Dec 26, 2004:

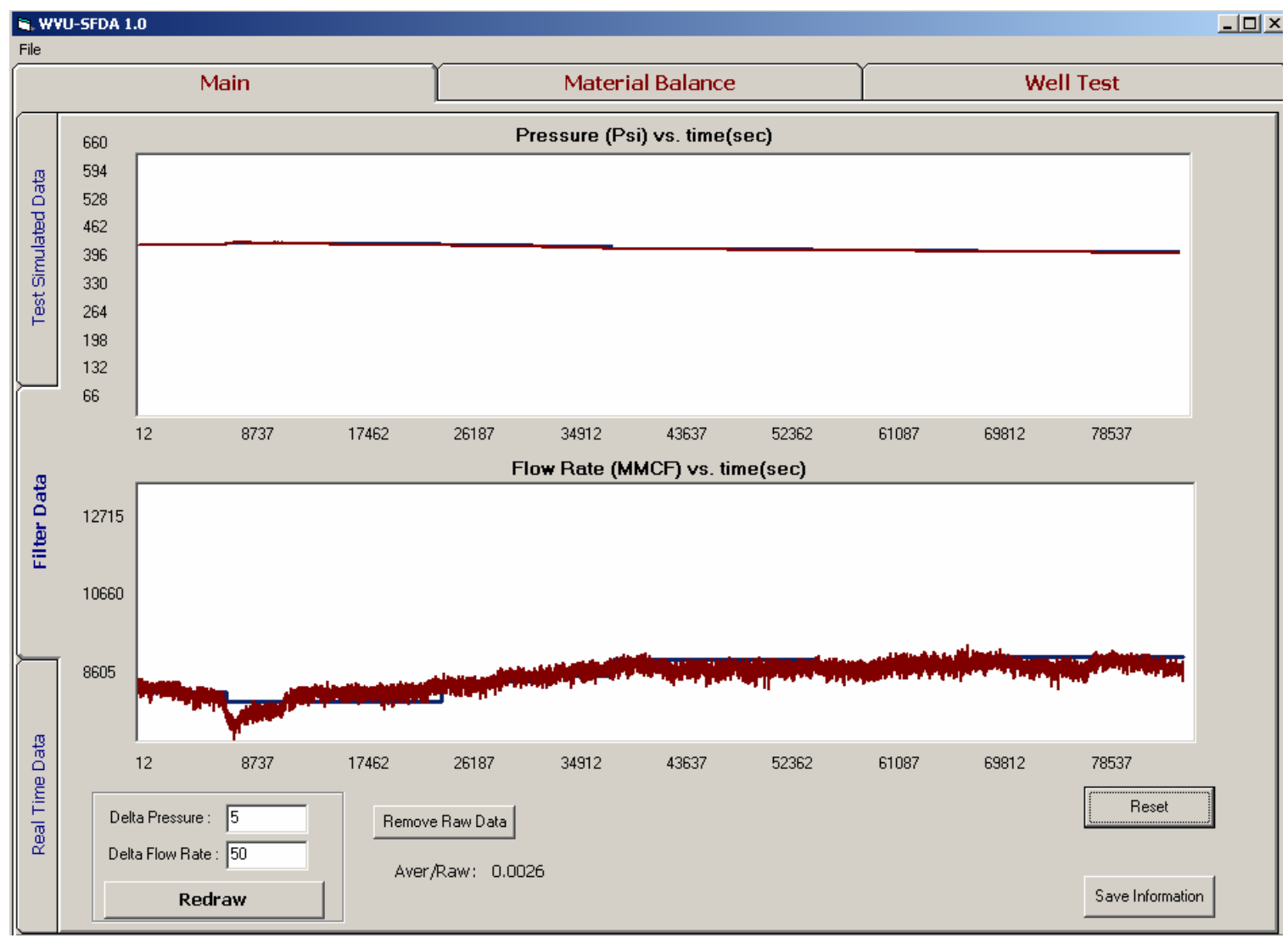

Figure 4.16- Pressure and Flow Rate- Actual and Summarized Data (Dec 26, 04) 
Showing only the plots for the summarized data for the same day:

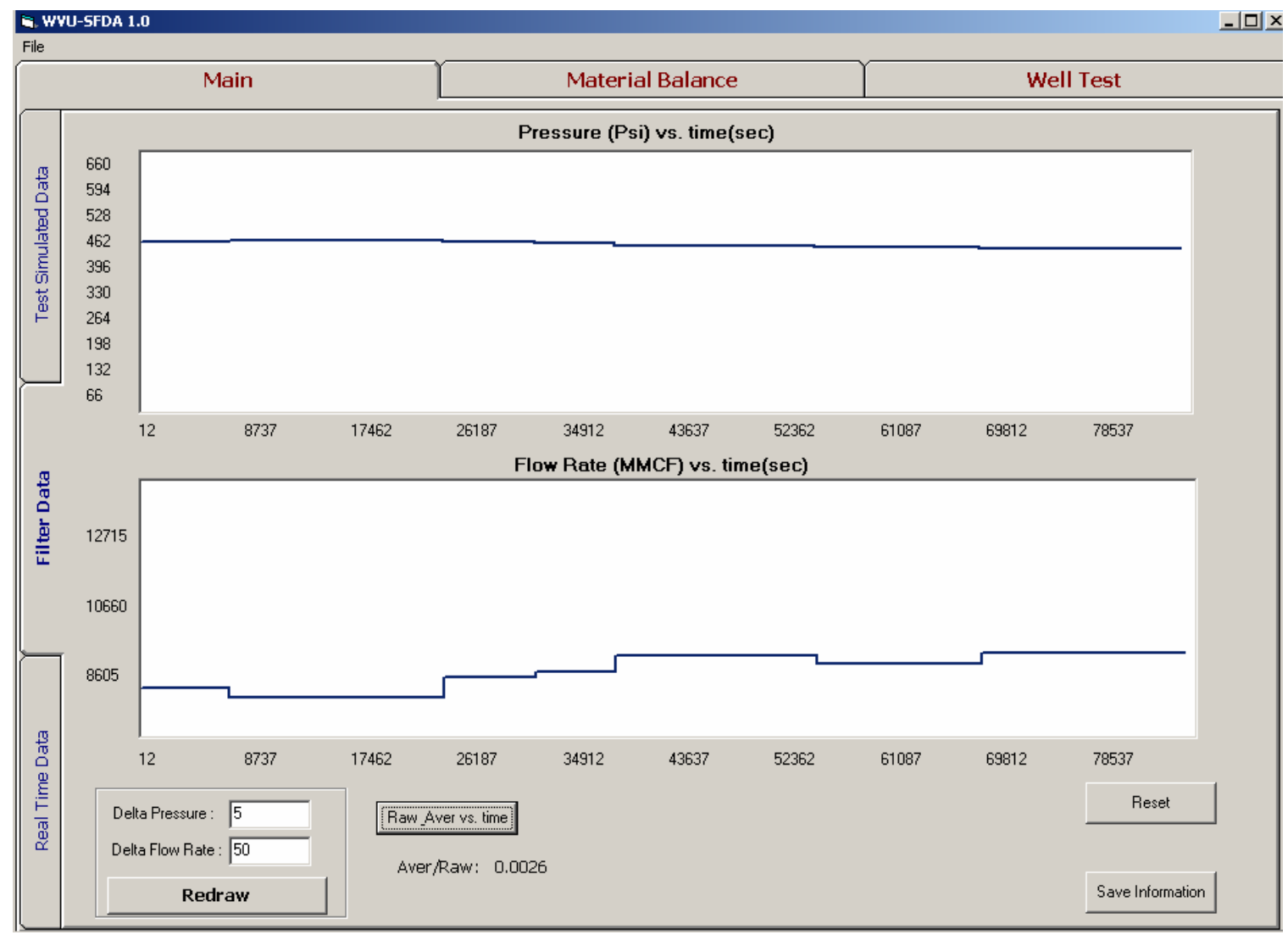

Figure 4.17-Pressure and Flow Rate -Summarized Data (Dec 26, 04)

As it can be seen, the summarized data does not look the same as the actual data. The preset values for pressure and flow rate can be changed and the graph redrawn until both the actual and the summarized plots match. After changing the preset values for this example, the following result was obtained in which the plot related to the summarized data contains $24 \%$ of the actual values ( $76 \%$ values are discarded). 


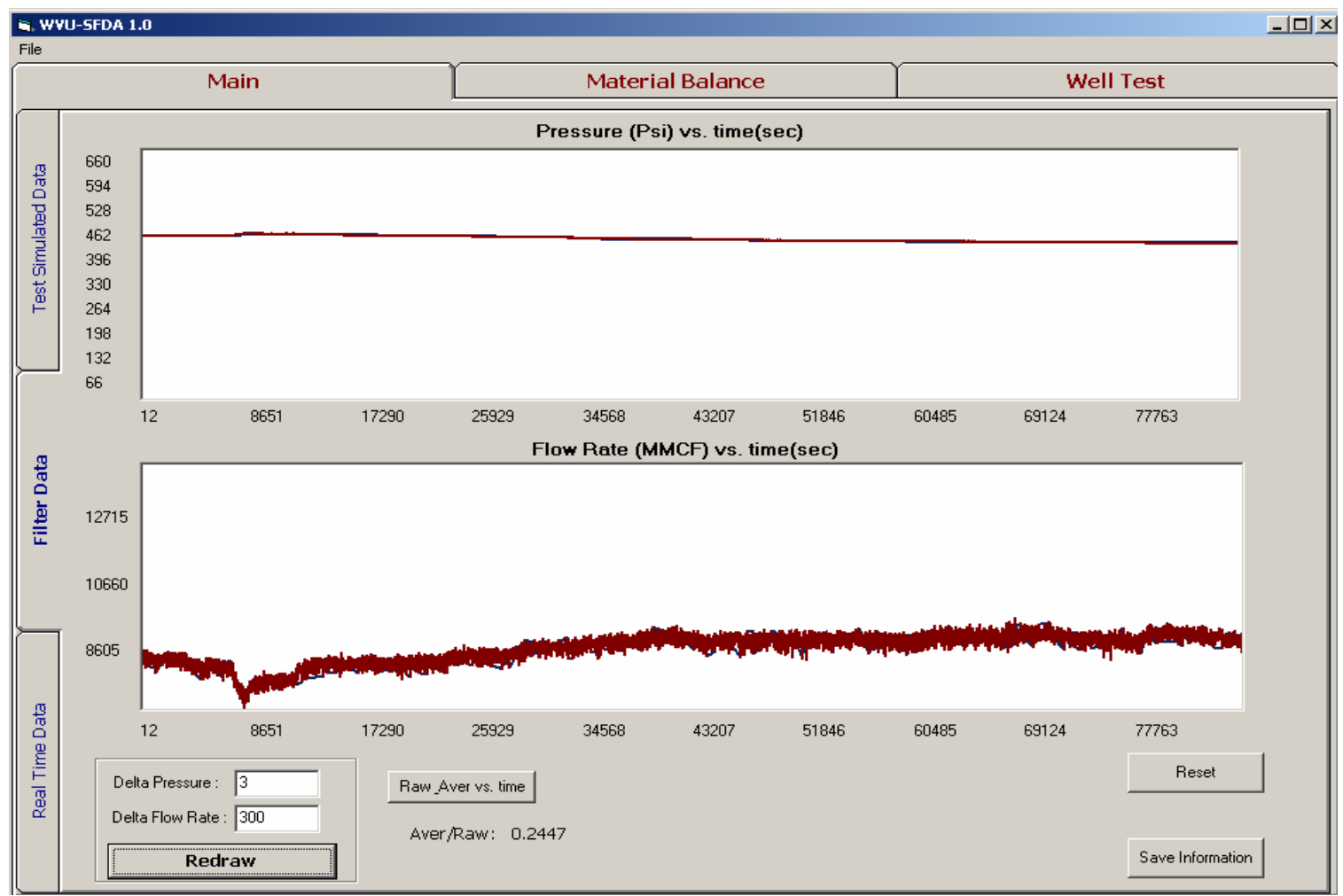

Figure 4.18- Pressure and Flow Rate- Actual and Summarized Data (Dec 26, 04)-Preset Changed

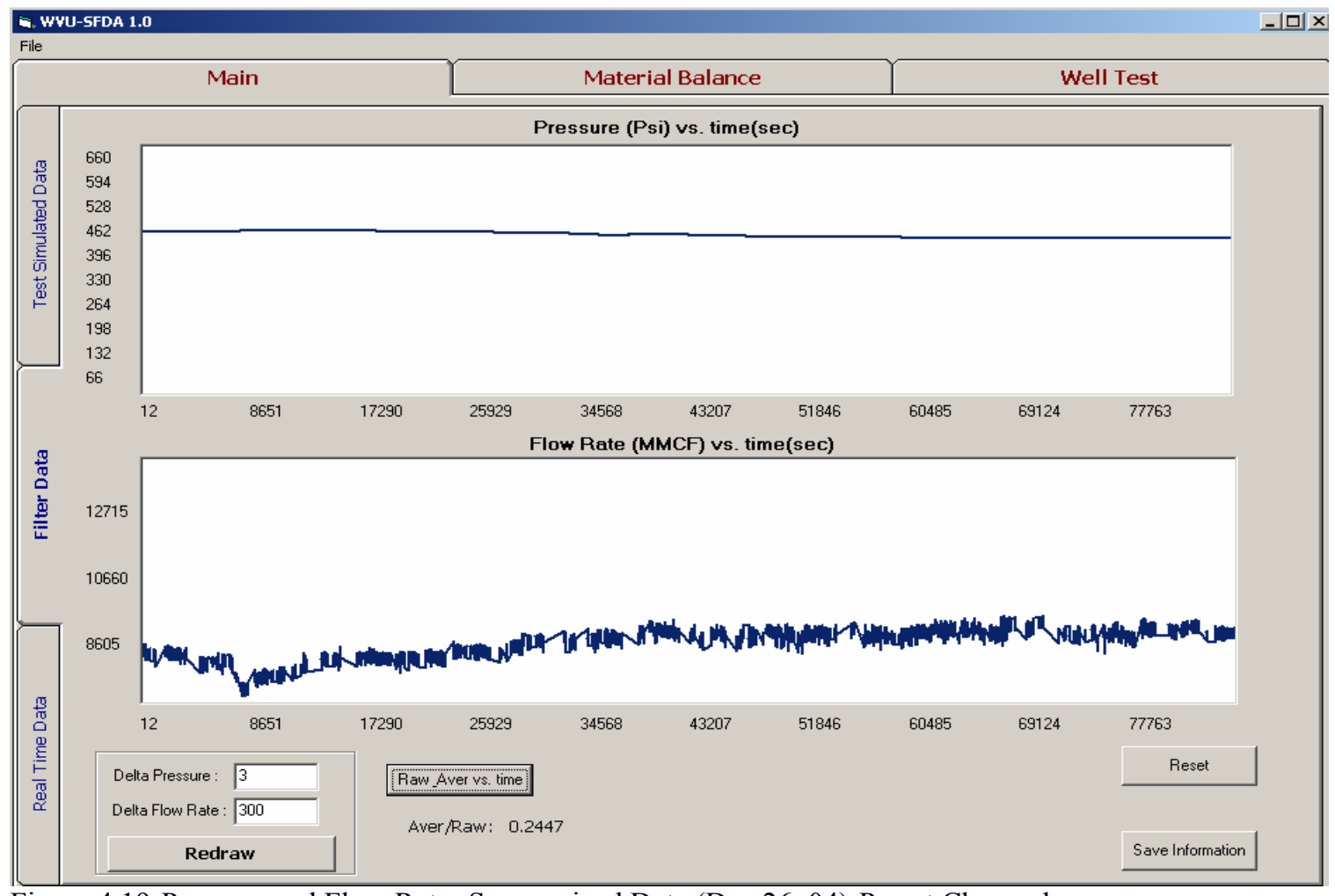

Figure 4.19-Pressure and Flow Rate -Summarized Data (Dec 26, 04)-Preset Changed 
Another example (March 15, 2005), in which the summarized data and actual data do not look the same, the preset values can be changed as many time as needed until both the summarized and actual information match:

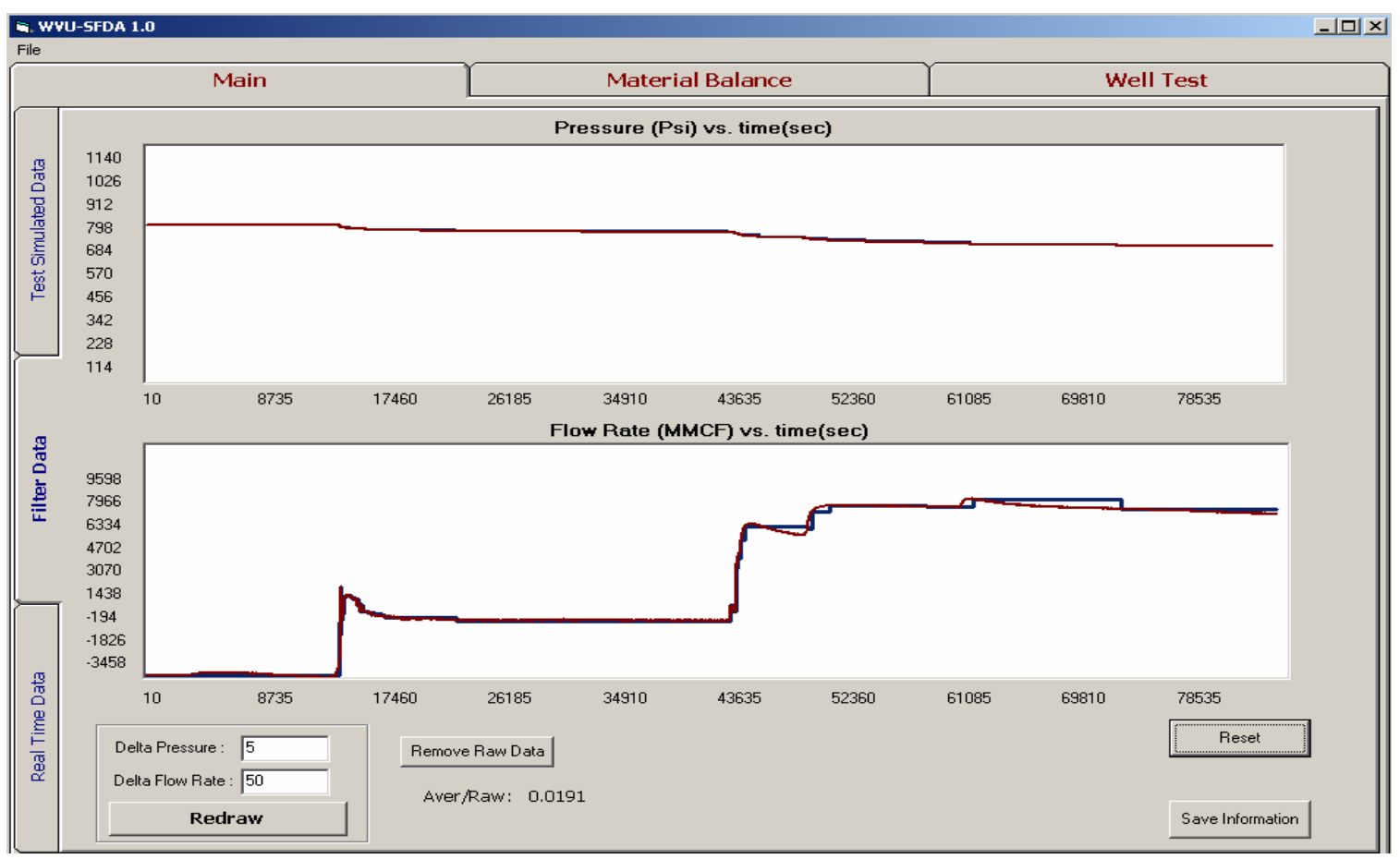

Figure 4.20- Pressure and Flow Rate- Actual and Summarized Data (March 15, 05)

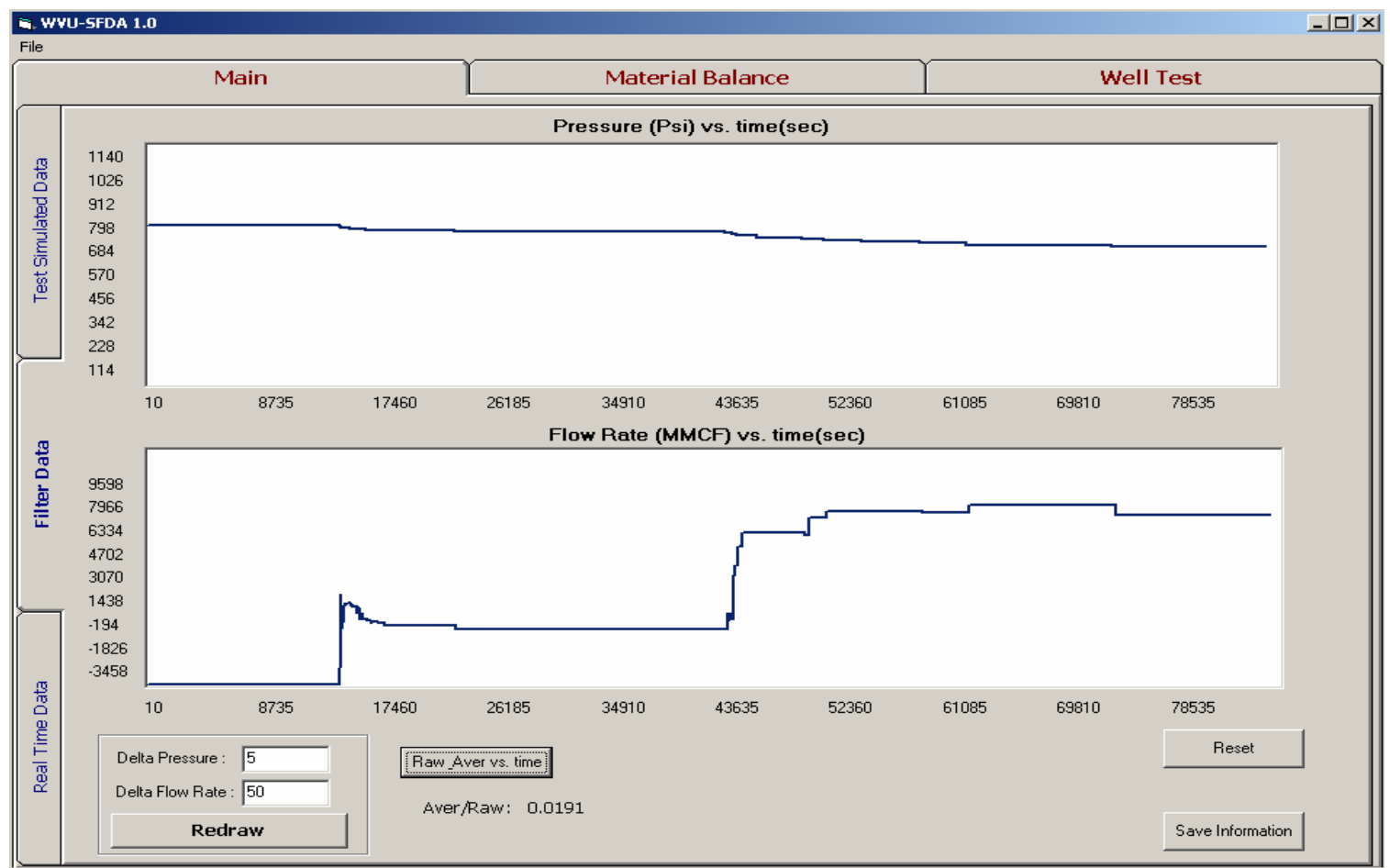

Figure 4.21-Pressure and Flow Rate -Summarized Data (March 15, 05) 


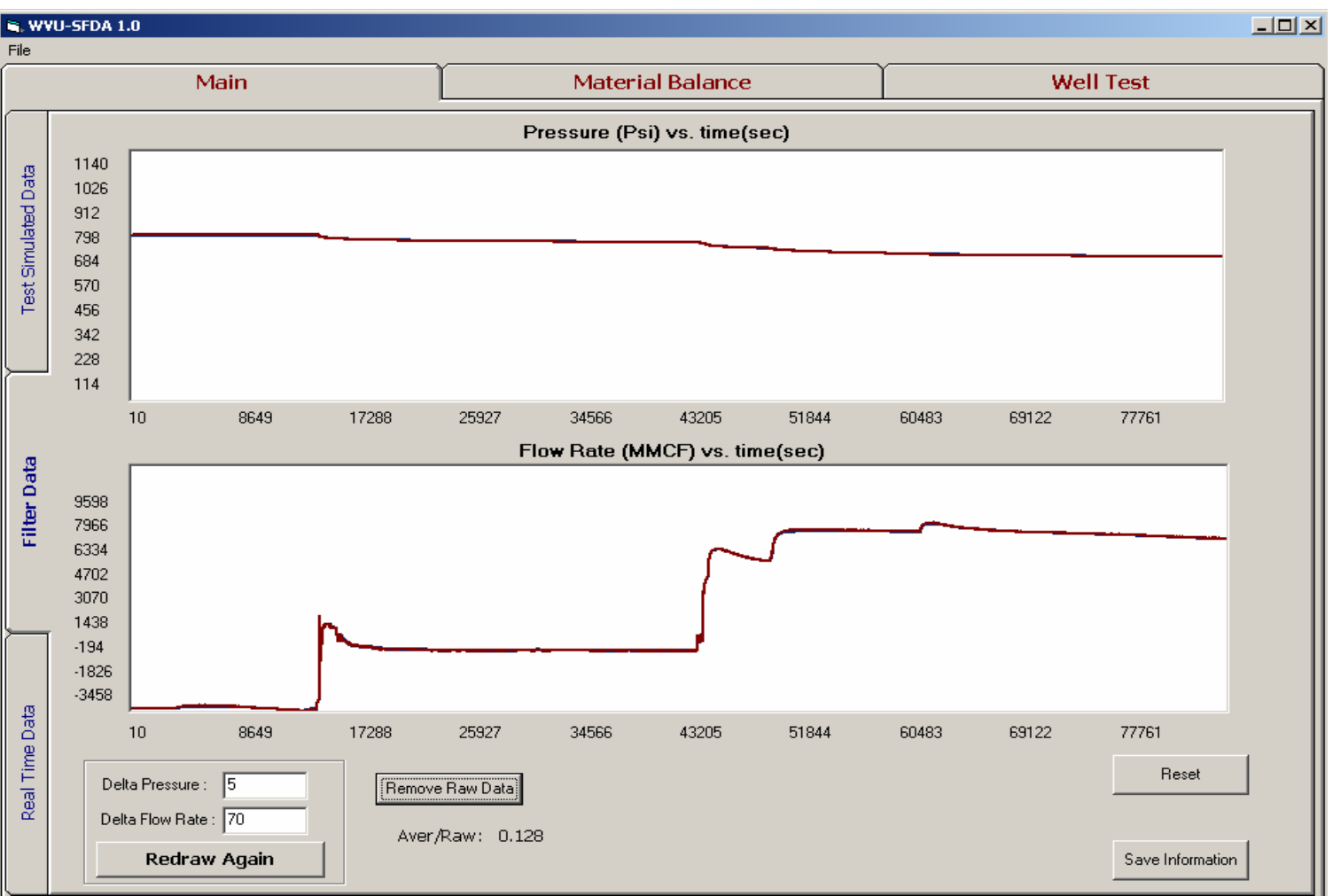

Figure 4.22- Pressure and Flow Rate- Actual and Summarized Data (March 15, 05)-Preset Changed

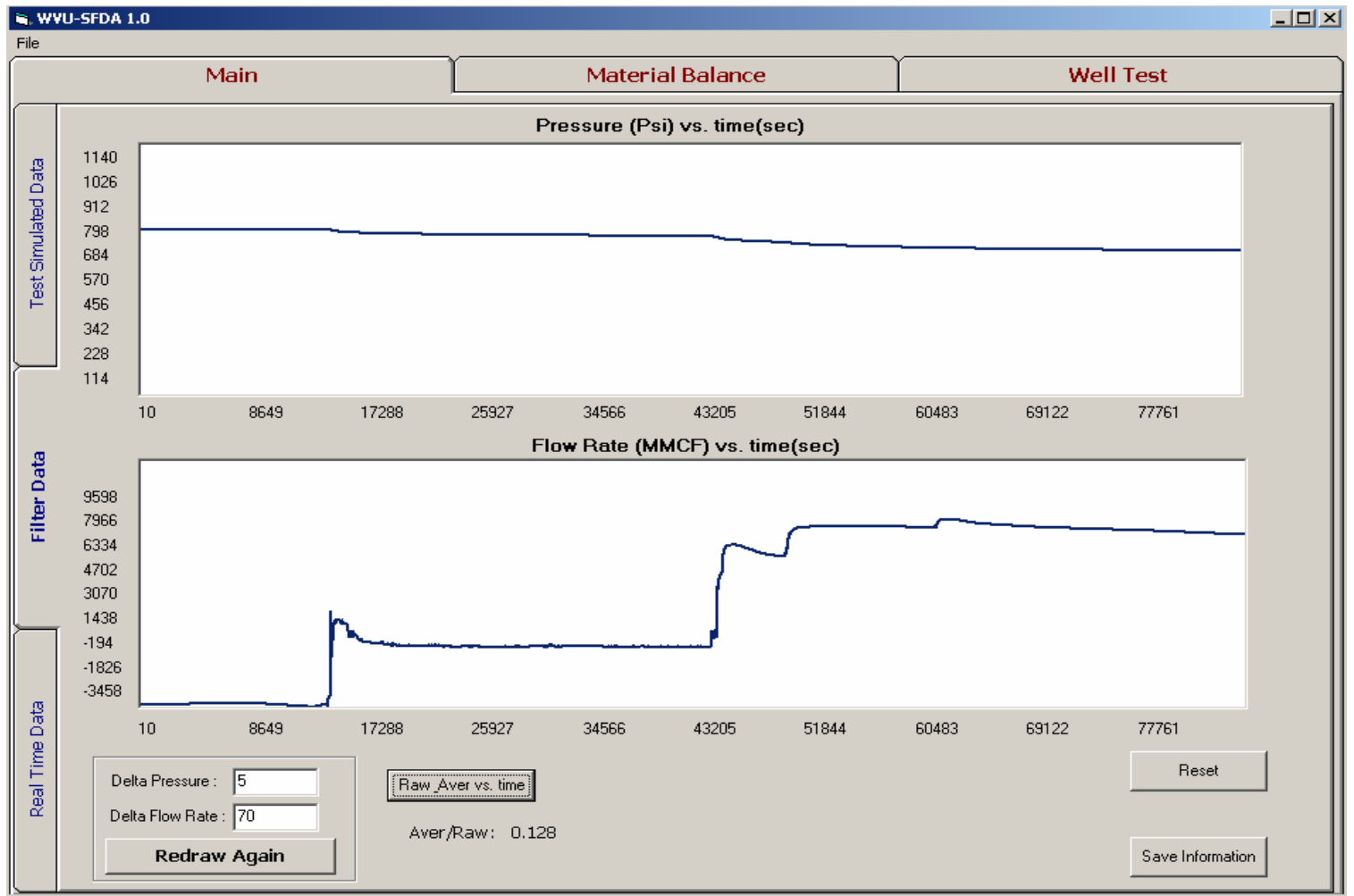

Figure 4.23-Pressure and Flow Rate -Summarized Data (March 15, 05)-Preset Changed 


\subsection{Material Balance Analysis}

After the data is summarized for daily periods, SFDA will allow creating monthly files to observe the changes in pressure, flow rate, and gas in place over such a period of time.

Material Balance Analysis was performed on the summarized data to calculate gas in place profile for monthly periods. The aim is to make the software capable of monitoring gas in place and detecting the presence of leaks in the reservoir. The following graphs represent the pressure, flow rate, and gas in place profile over a month in blue, red, and black colors respectively. In addition, the changes in pressure, flow rate, and gas in place can be observed from the upper part of the graphs (the graphs in gray represents the changes in such parameters). SFDA will perform the calculations according to the input values of cumulative production, Pressure 1 and Pressure 2:

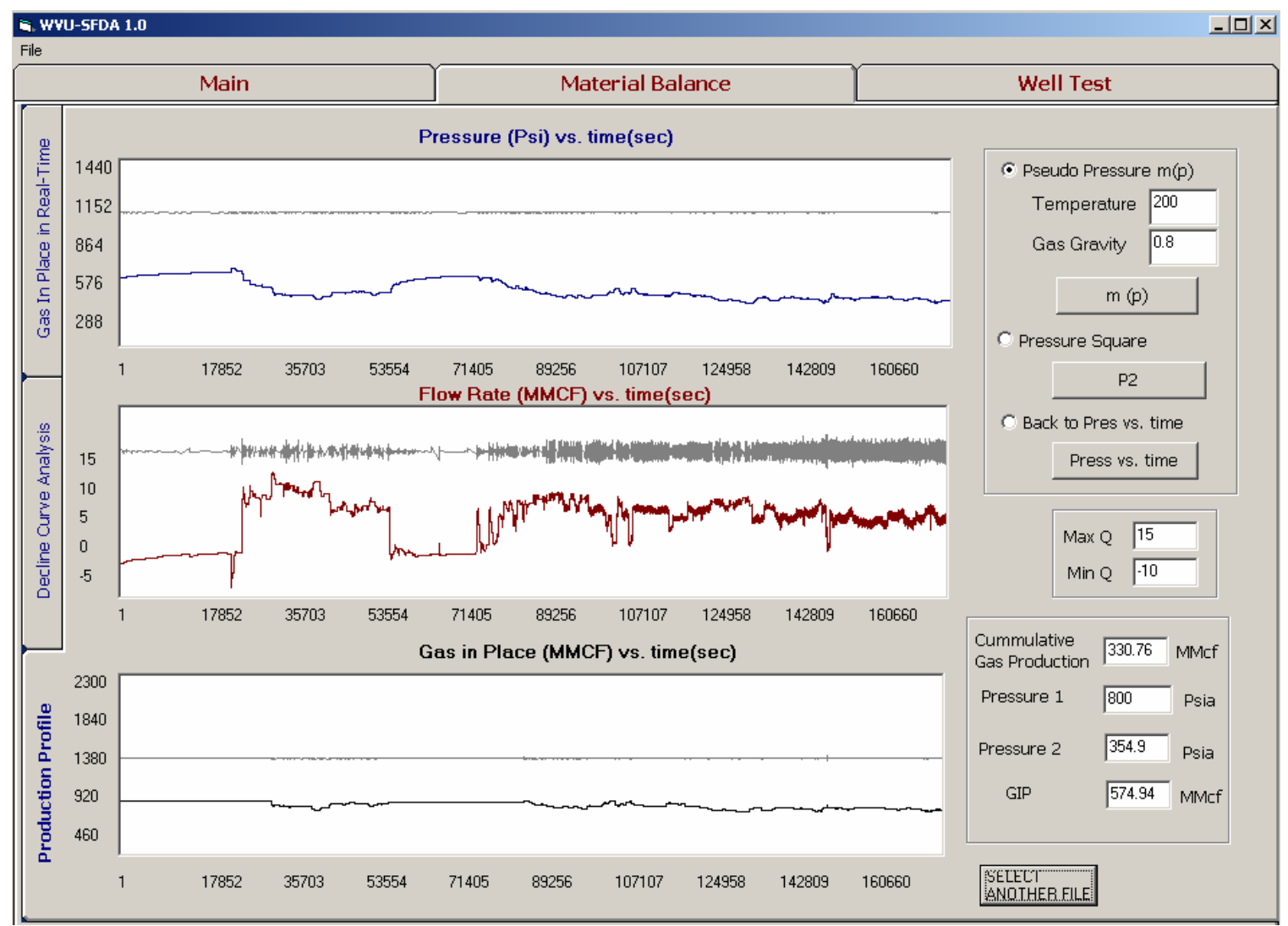

Figure 4.24-Pressure, Flow Rate, and Gas in Place Profile-Summarized Data January 2005 


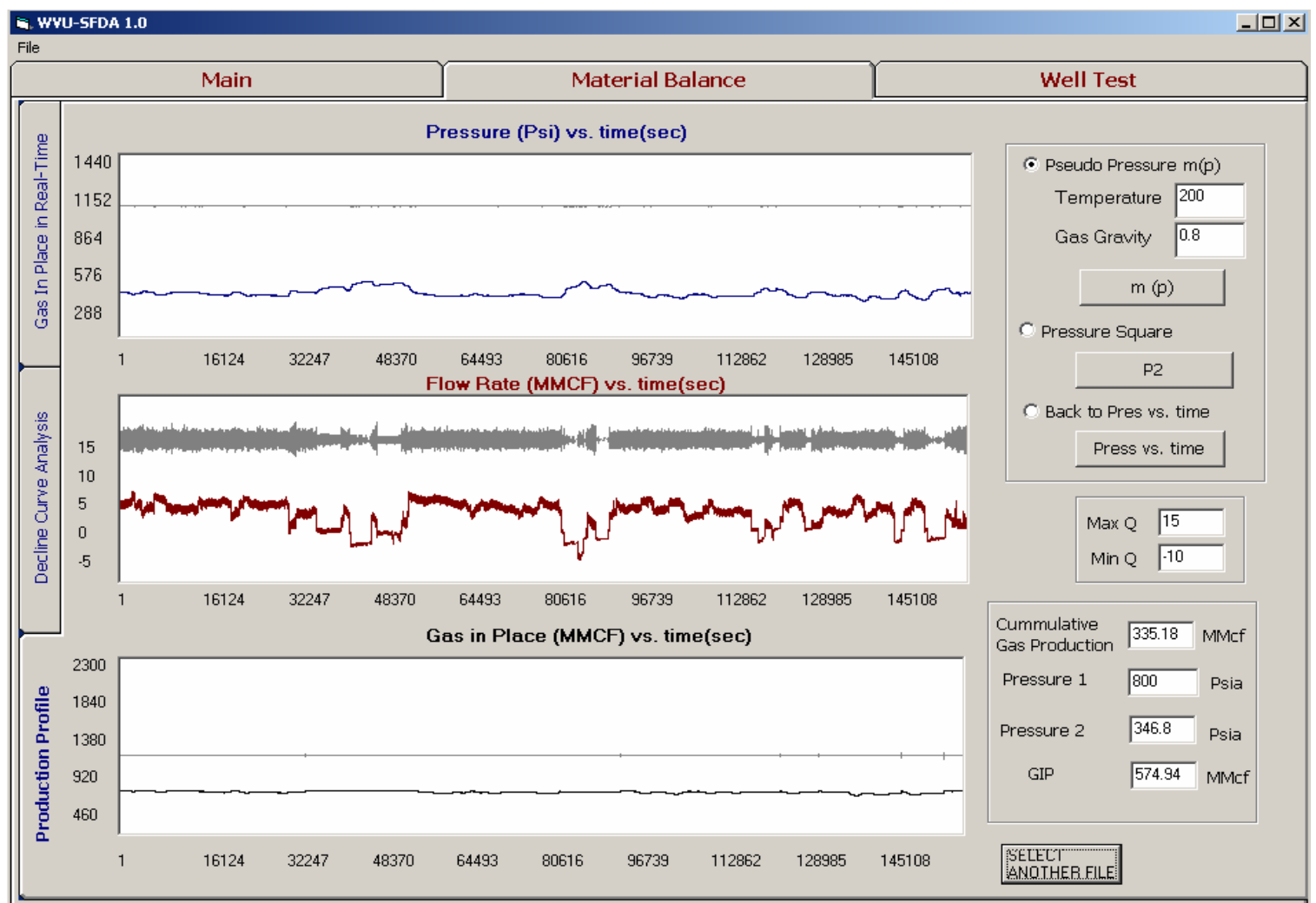

Figure 4.25-Pressure, Flow Rate, and Gas in Place Profile-Summarized Data February 2005

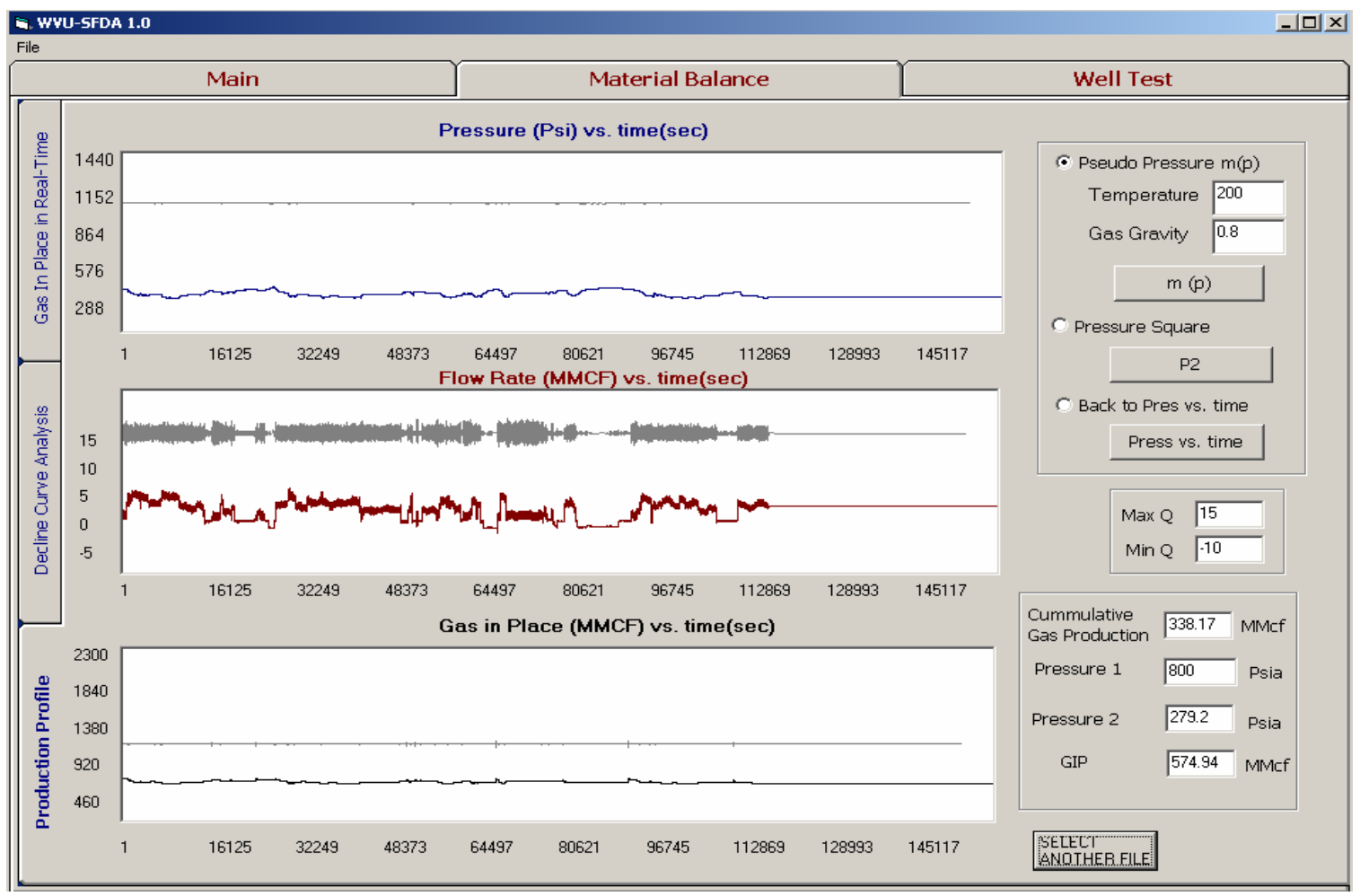

Figure 4.26-Pressure, Flow Rate, and Gas in Place Profile-Summarized Data March 2005 


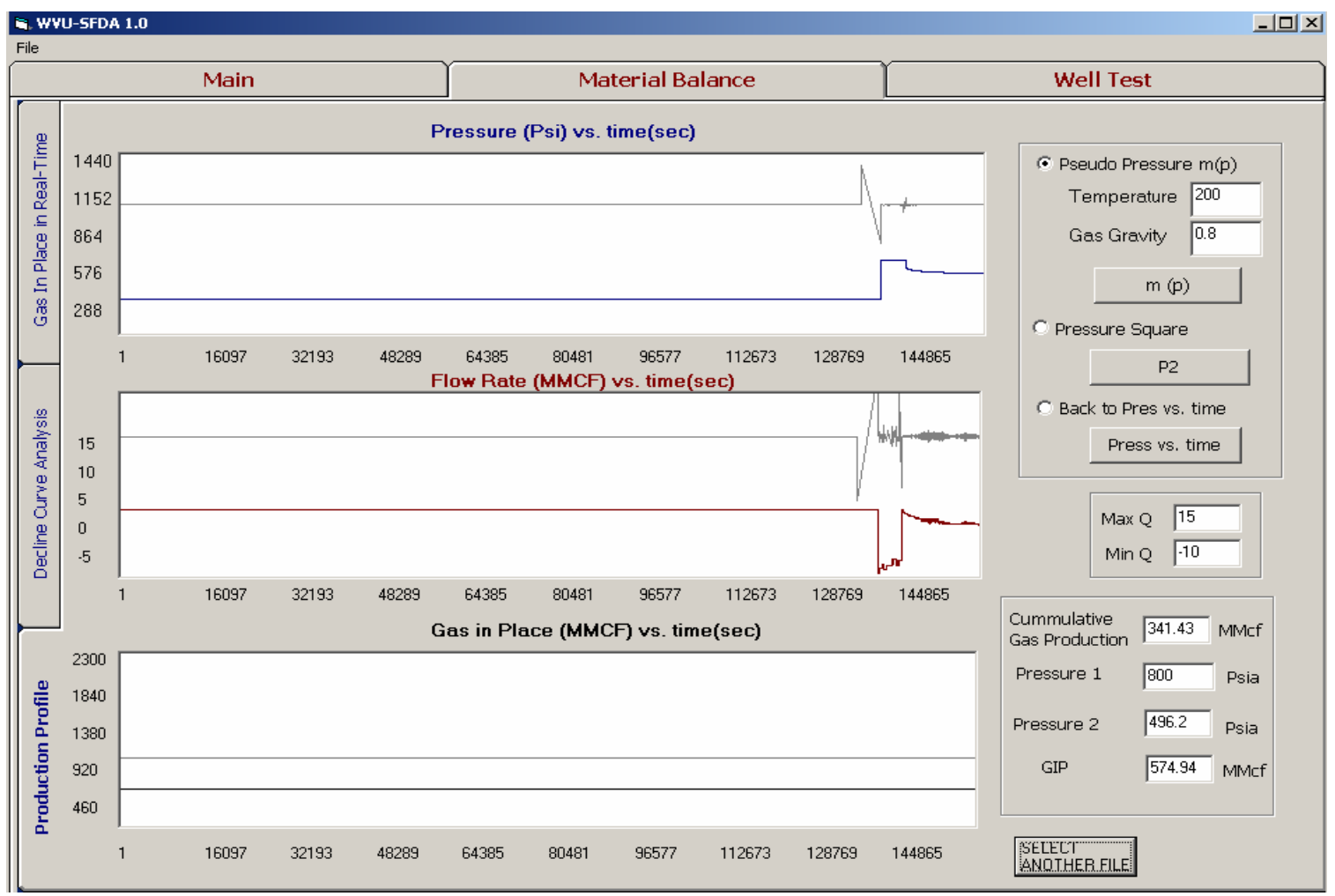

Figure 4.27-Pressure, Flow Rate, and Gas in Place Profile-Summarized Data April 2005

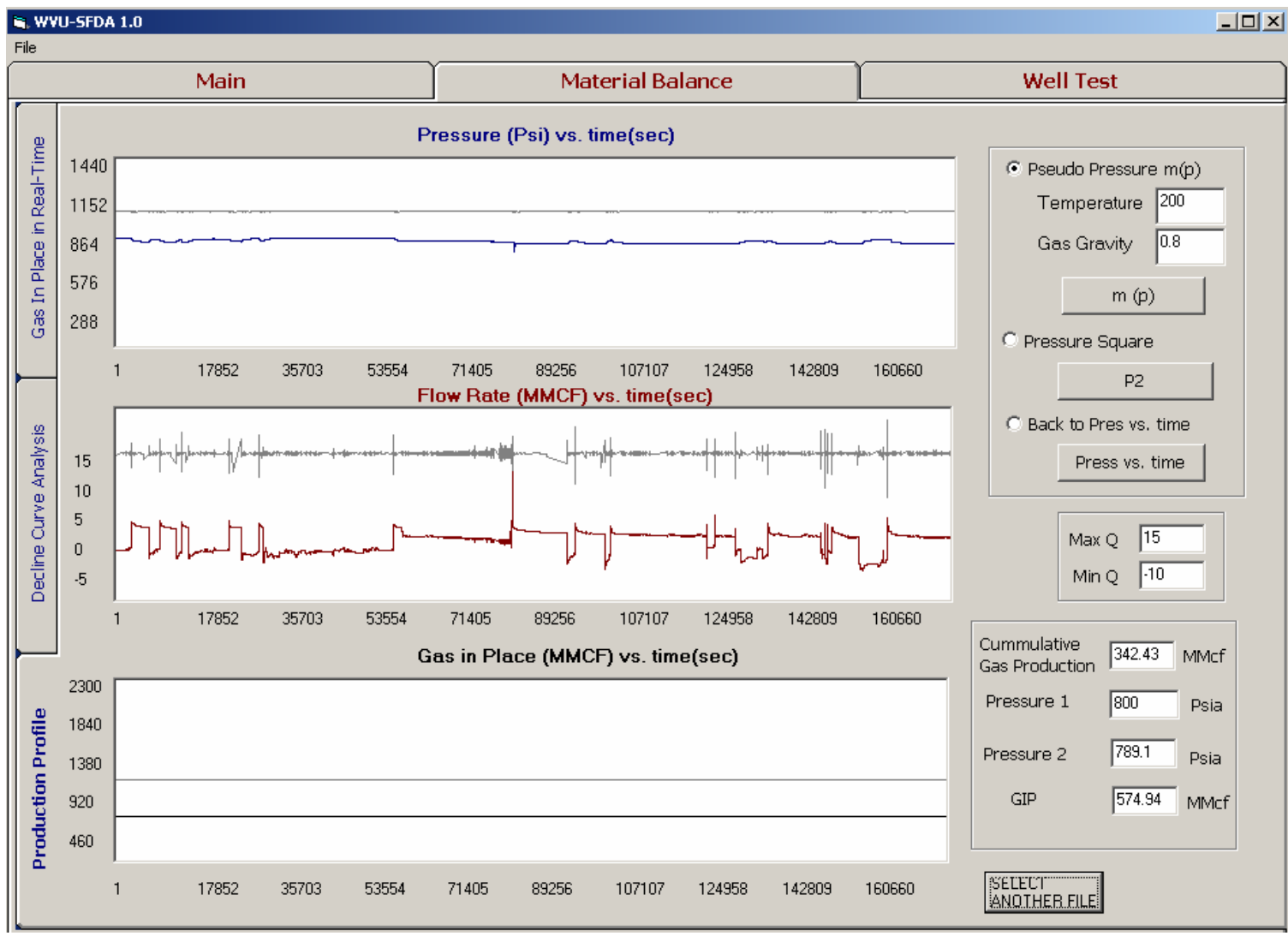

Figure 4.28-Pressure, Flow Rate, and Gas in Place Profile-Summarized Data October 2004 


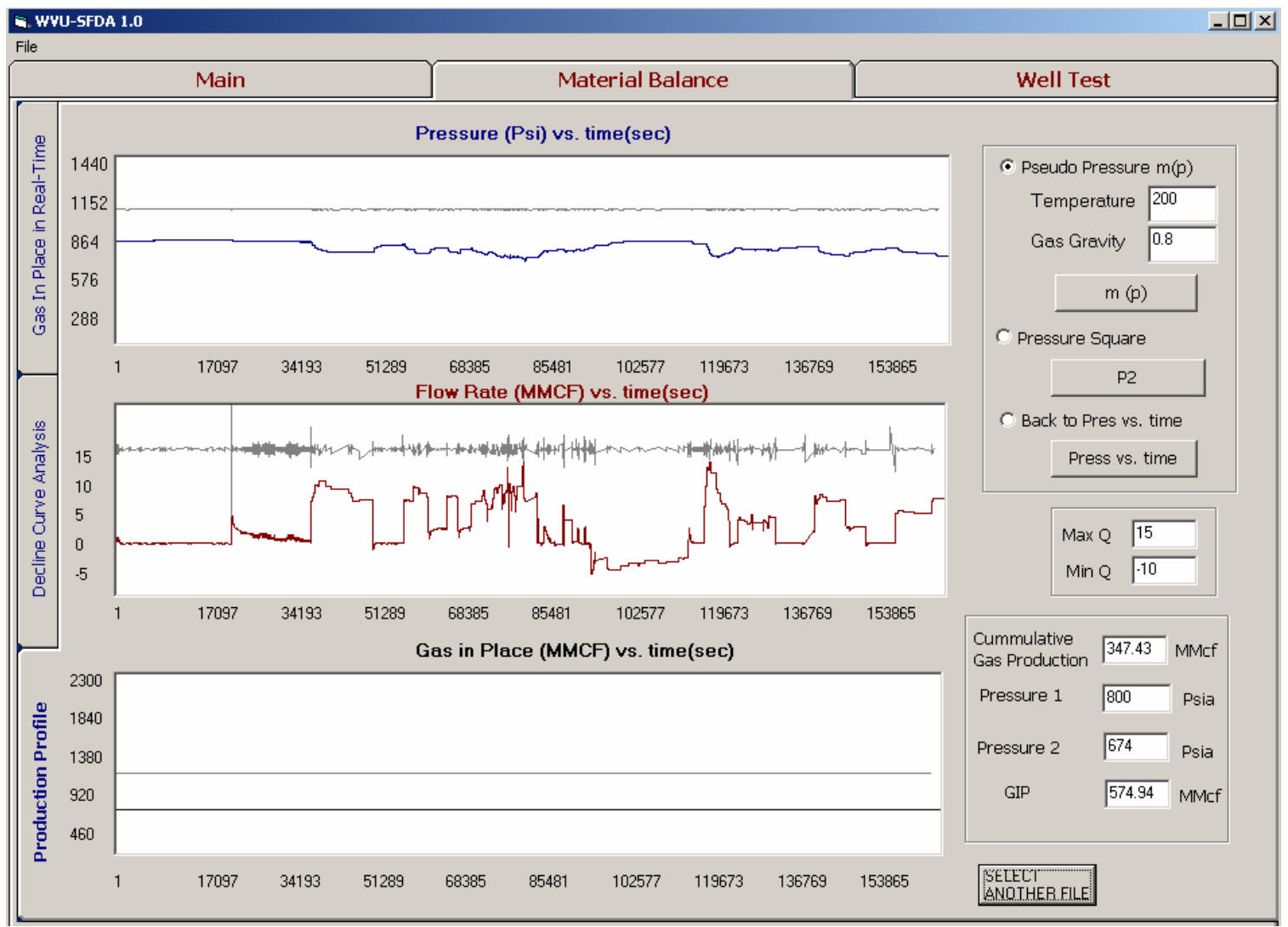

Figure 4.29-Pressure, Flow Rate, and Gas in Place Profile-Summarized Data November 2004 


\subsubsection{Gas in Place in Real Time}

Gas in place in Real-Time can be observed as data is entering the system. SFDA estimates the values of gas in place and shows it in a graph as the pressure and flow rate parameters are shown by the program. The following figure will demonstrate how the program shows the material balance calculations in Real-Time:

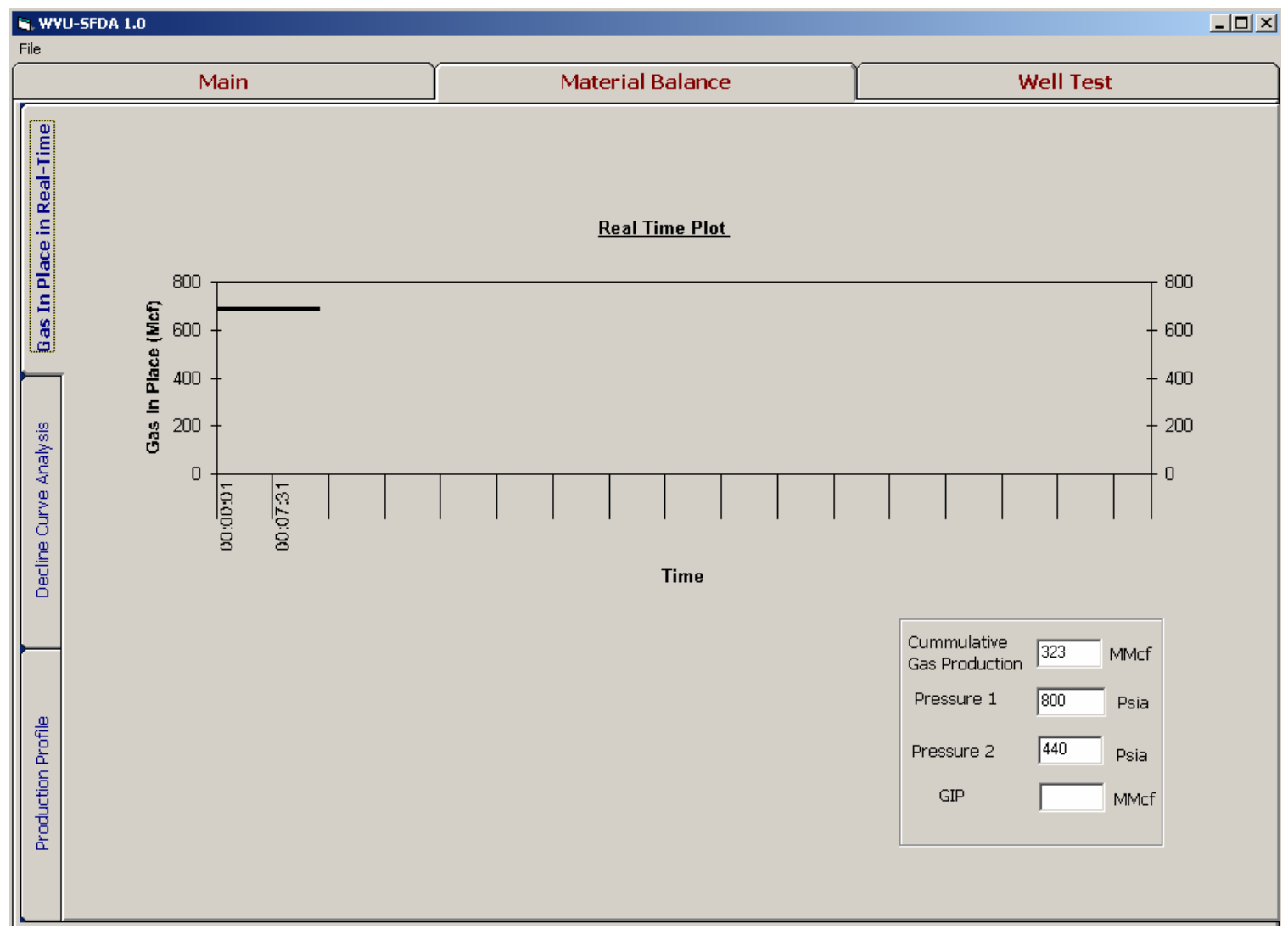

Figure 4.30-Gas in Place in Real-Time 


\subsection{Pseudo Pressure Analyses}

SFDA has the option of changing the plot of pressure versus time to pseudo-pressure versus time. In several cases during pressure transient analysis for gas production it is recommended to perform the well testing analysis using pseudo pressure values. The well test section of the software will allow the user to plot pseudo pressure versus pressure and draw the Horner plot from an area selected by the user.

The following figures show the summarized data from January 05 where the pressure profile has been converted into pseudo pressure profile.

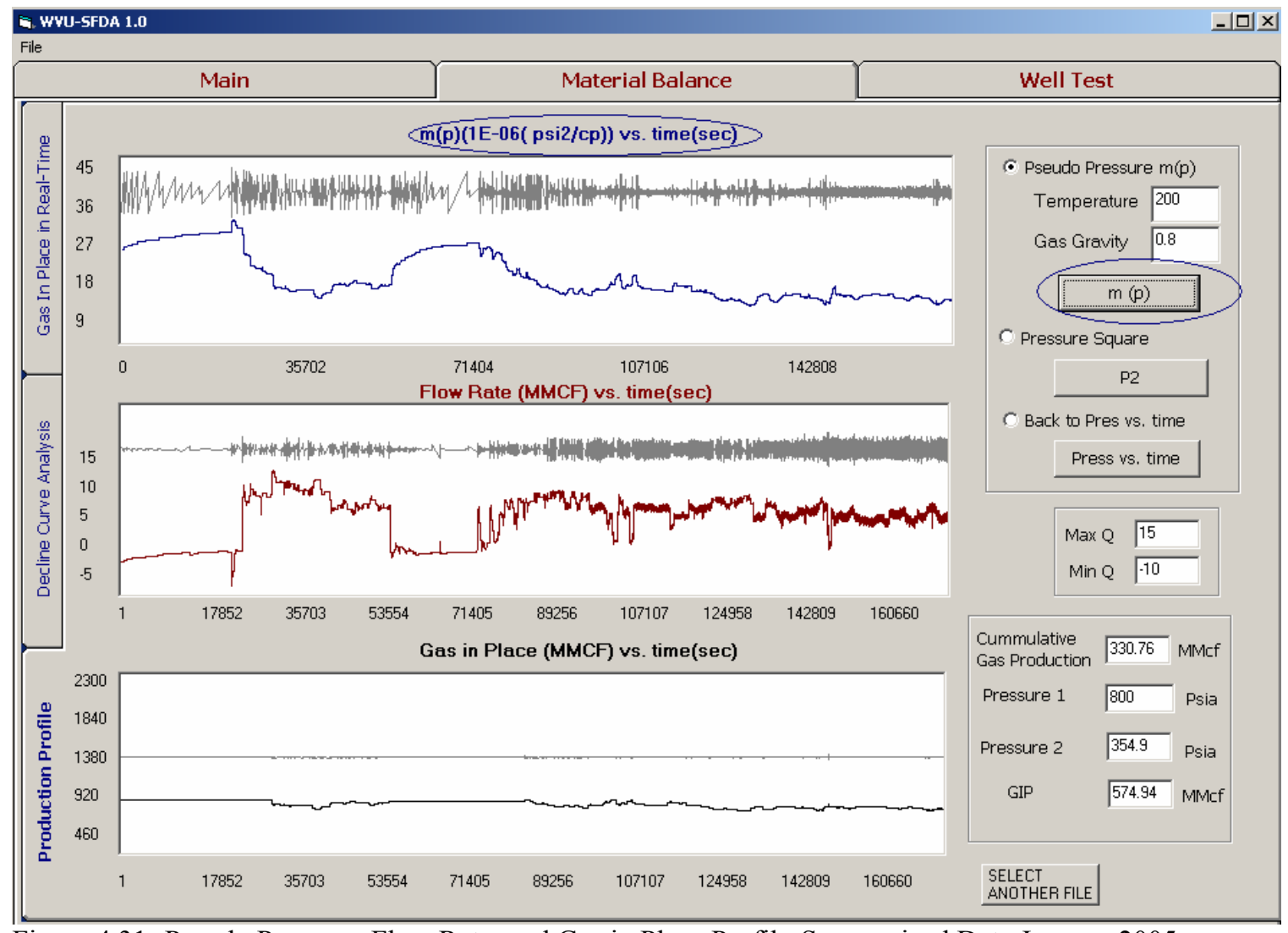

Figure 4.31- Pseudo Pressure, Flow Rate, and Gas in Place Profile-Summarized Data January 2005

Whenever a pressure transient period has been detected by the program, the user will have the option of plotting pseudo pressure versus pressure and selecting an area to draw the Horner plot. 


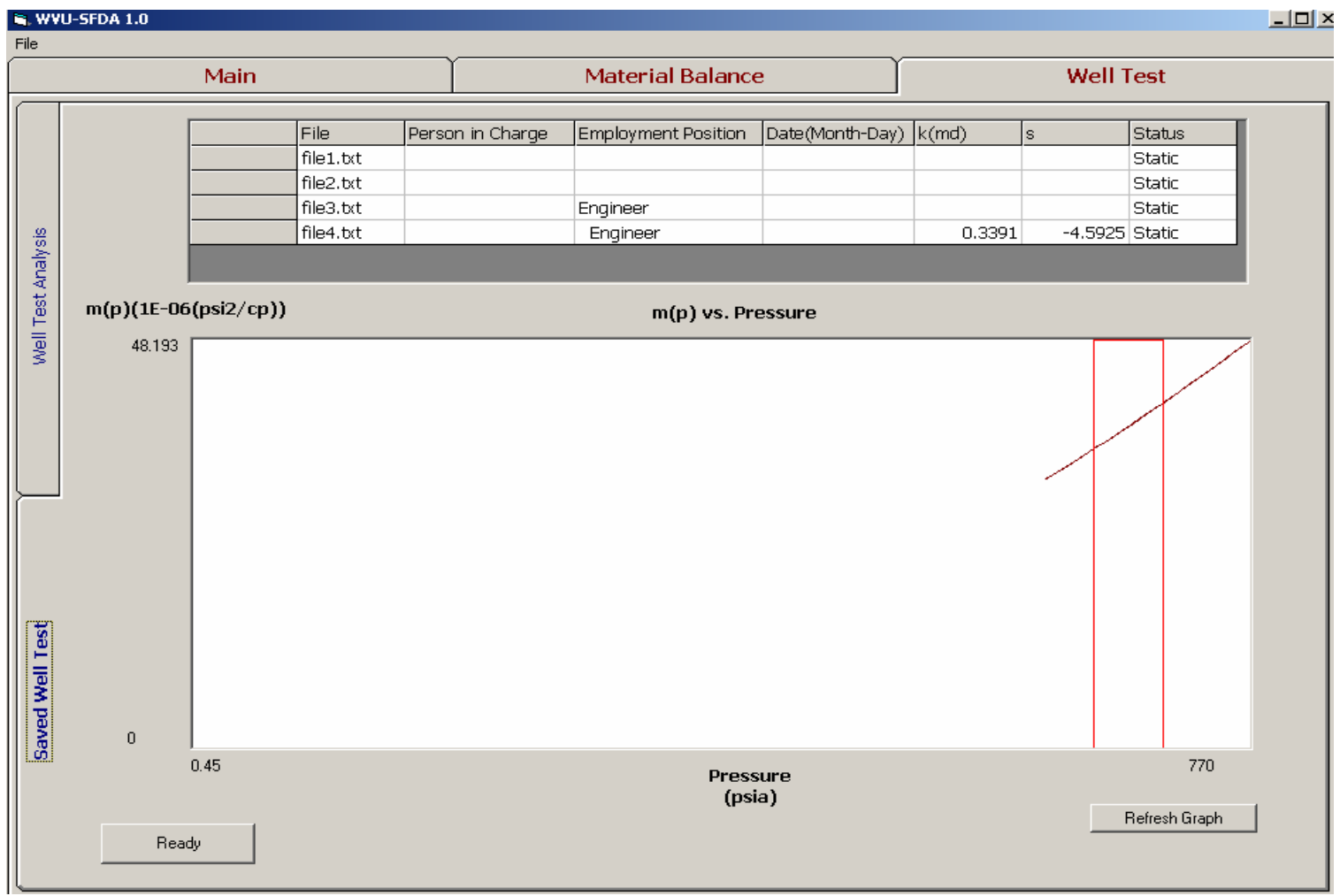

Figure 4.32-Area selection for Horner Plot-Pseudo Pressure Analysis

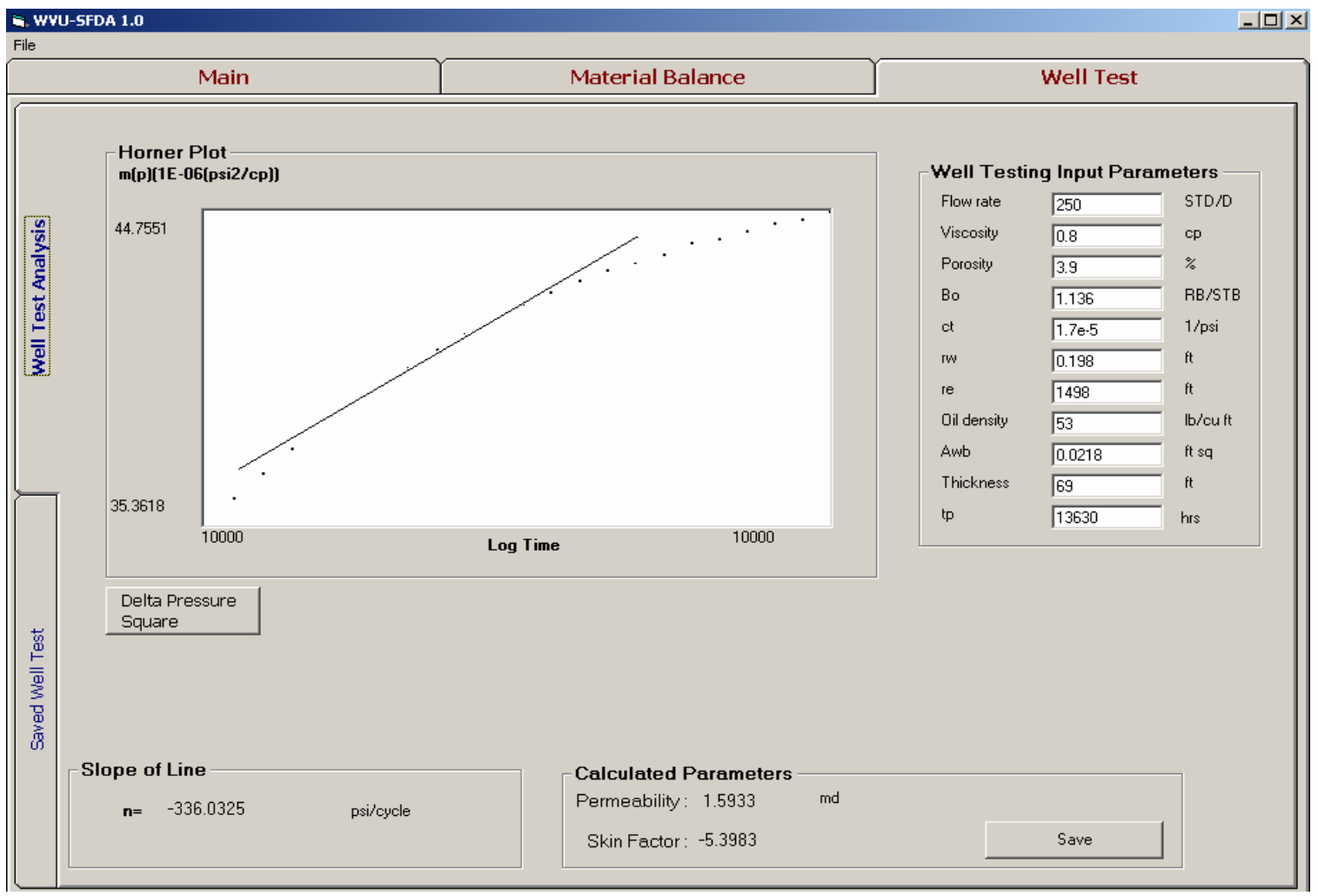

Figure 4.33- Horner Plot and Line Drawn to obtain permeability and skin factor-Pseudo Pressure Analysis 


\subsection{Pressure Square Analyses}

SFDA also has the option of changing the plot of pressure versus time to the square of pressure versus time. This is used in some well test analyses to obtain permeability and skin factor from drawdown periods. The following figure shows the pressure square from the real time data of January 2005.

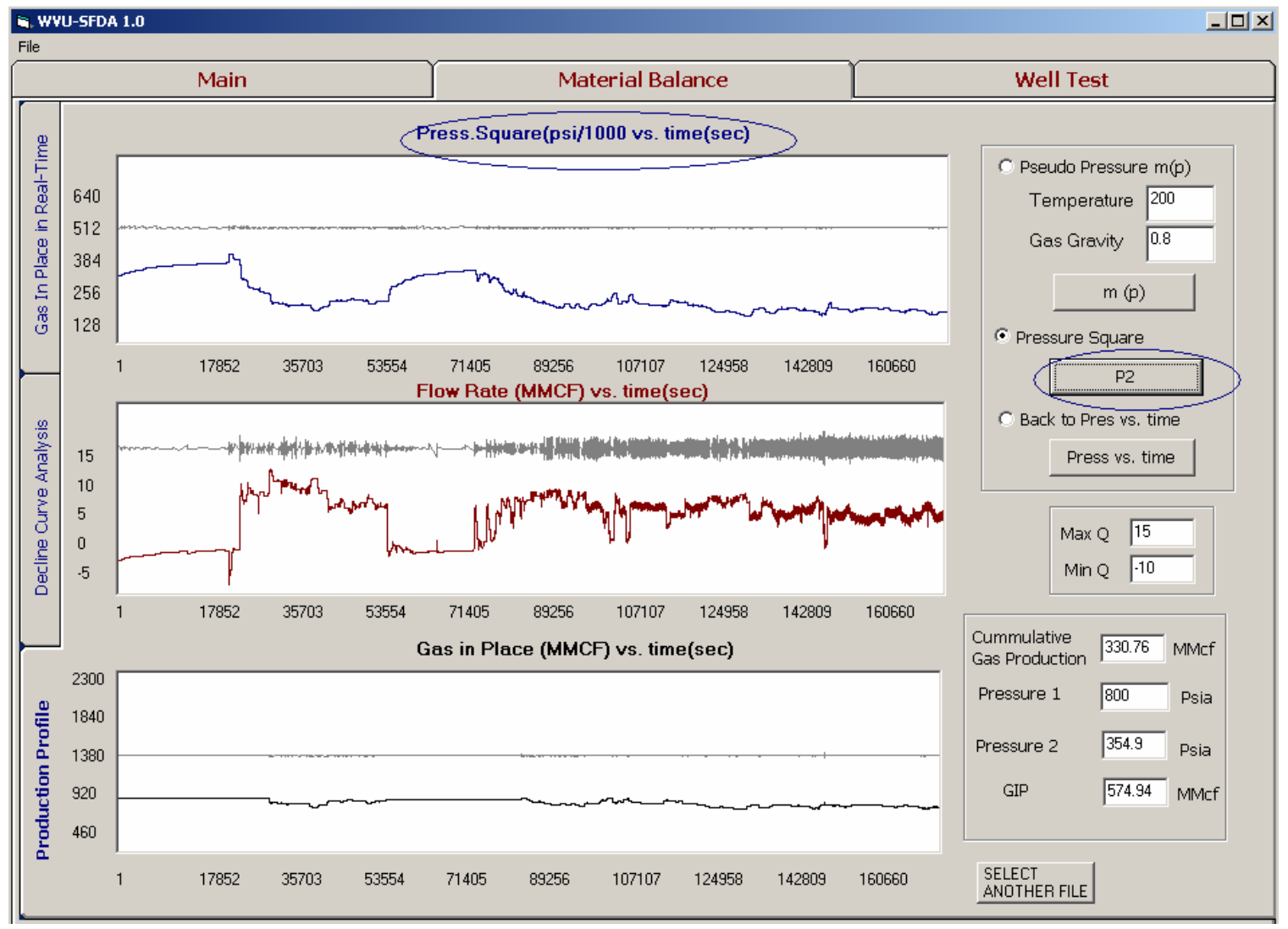

Figure 4.34- Pressure Square, Flow Rate, and Gas in Place Profile-Summarized Data January 2005

The user might choose to obtain permeability and skin from drawdown periods. The well test analysis window will have the option of drawing the Semi-Log plot using values of $\Delta \mathrm{P}^{2}$ versus time. 


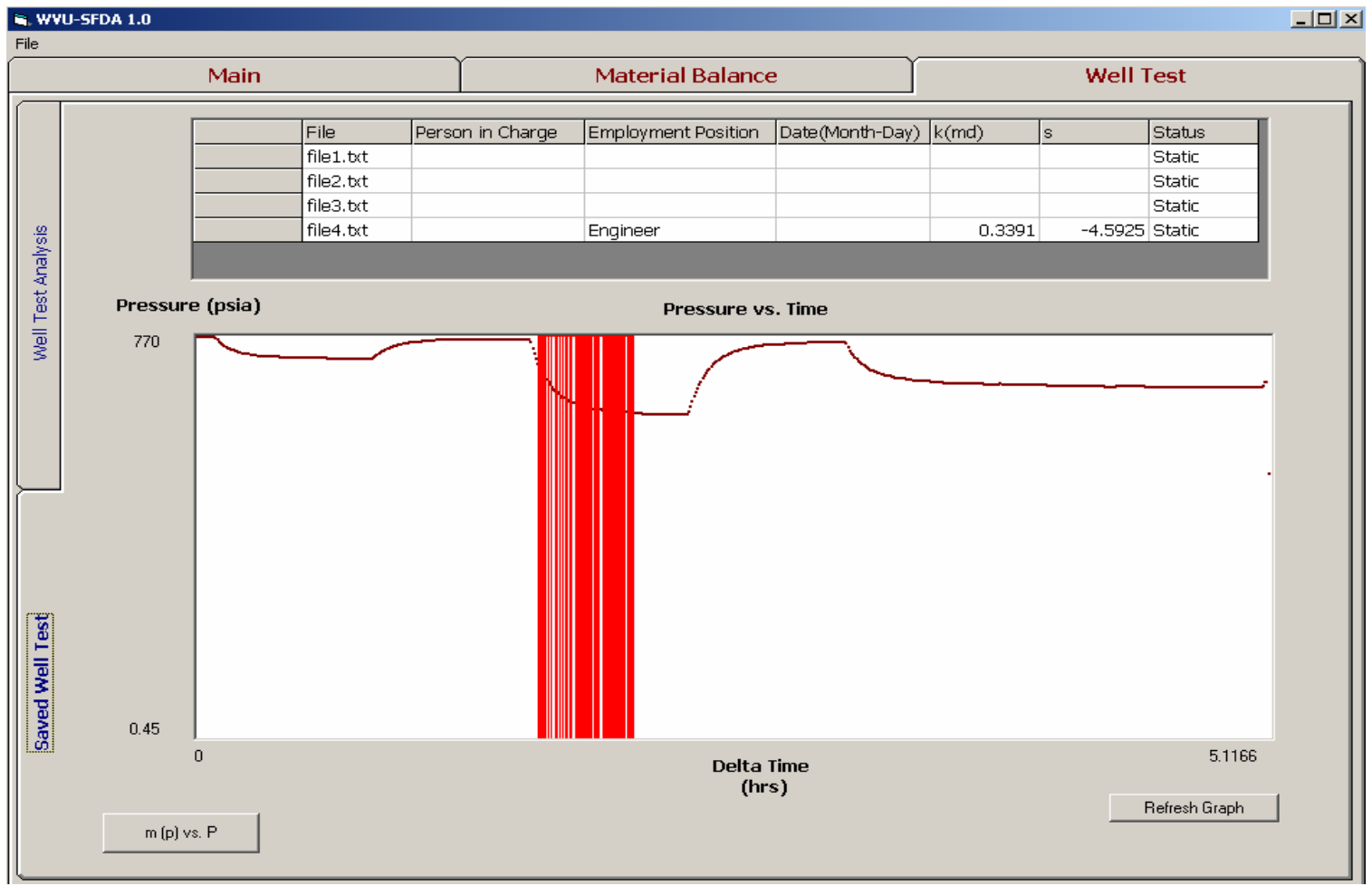

Figure 4.35-Area selection for Semi-Log Plot-Drawdown Period

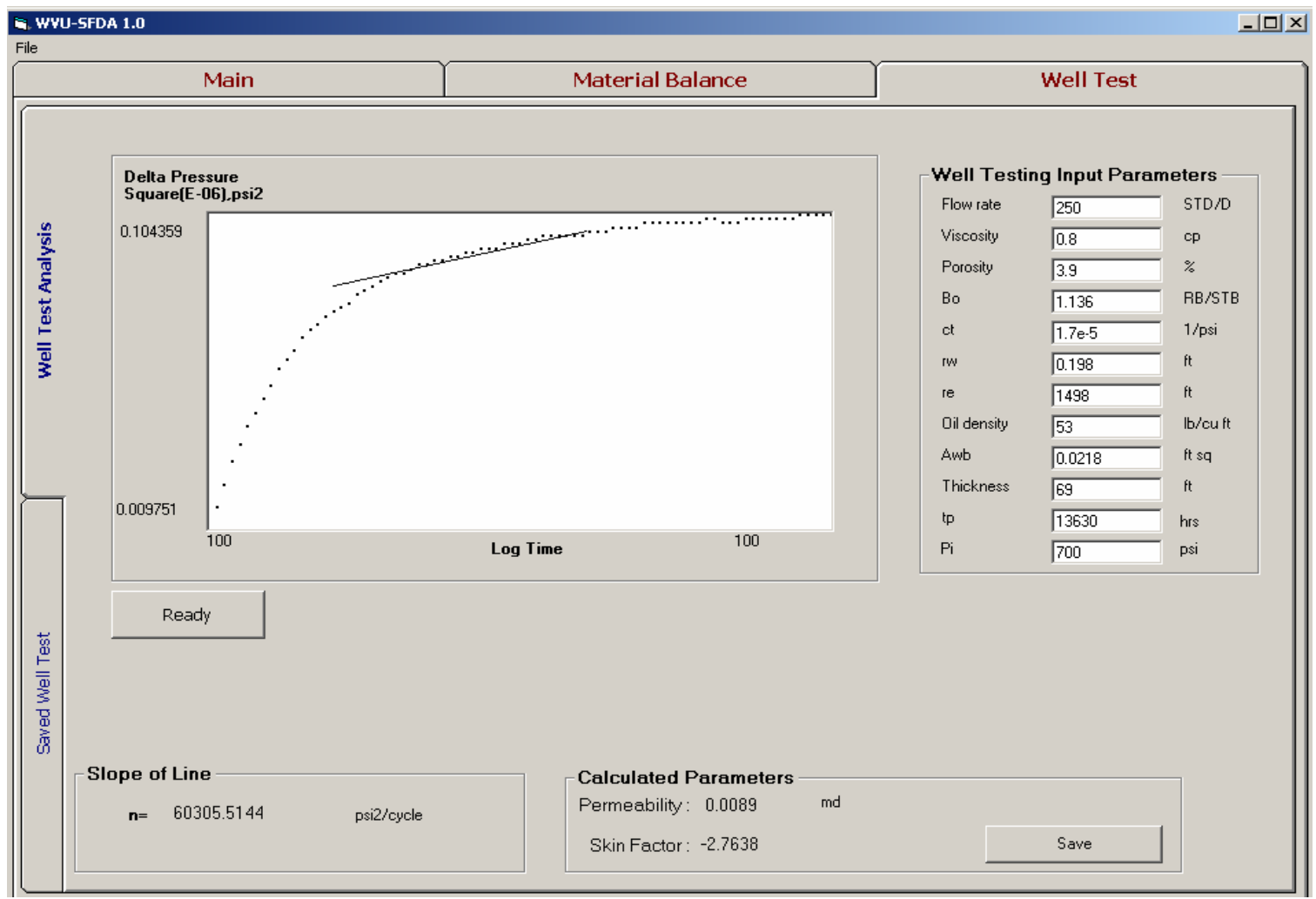

Figure 4.36- Semi-Log Plot and Line Drawn to obtain permeability and skin factor-Pressure Square Analysis 


\subsection{Decline Curve Analysis}

The following figures will show the results after performing decline curve analysis to the summarized data. SFDA is capable of performing two types of decline curve analysis: flow rate versus time, and flow rate versus cumulative production.

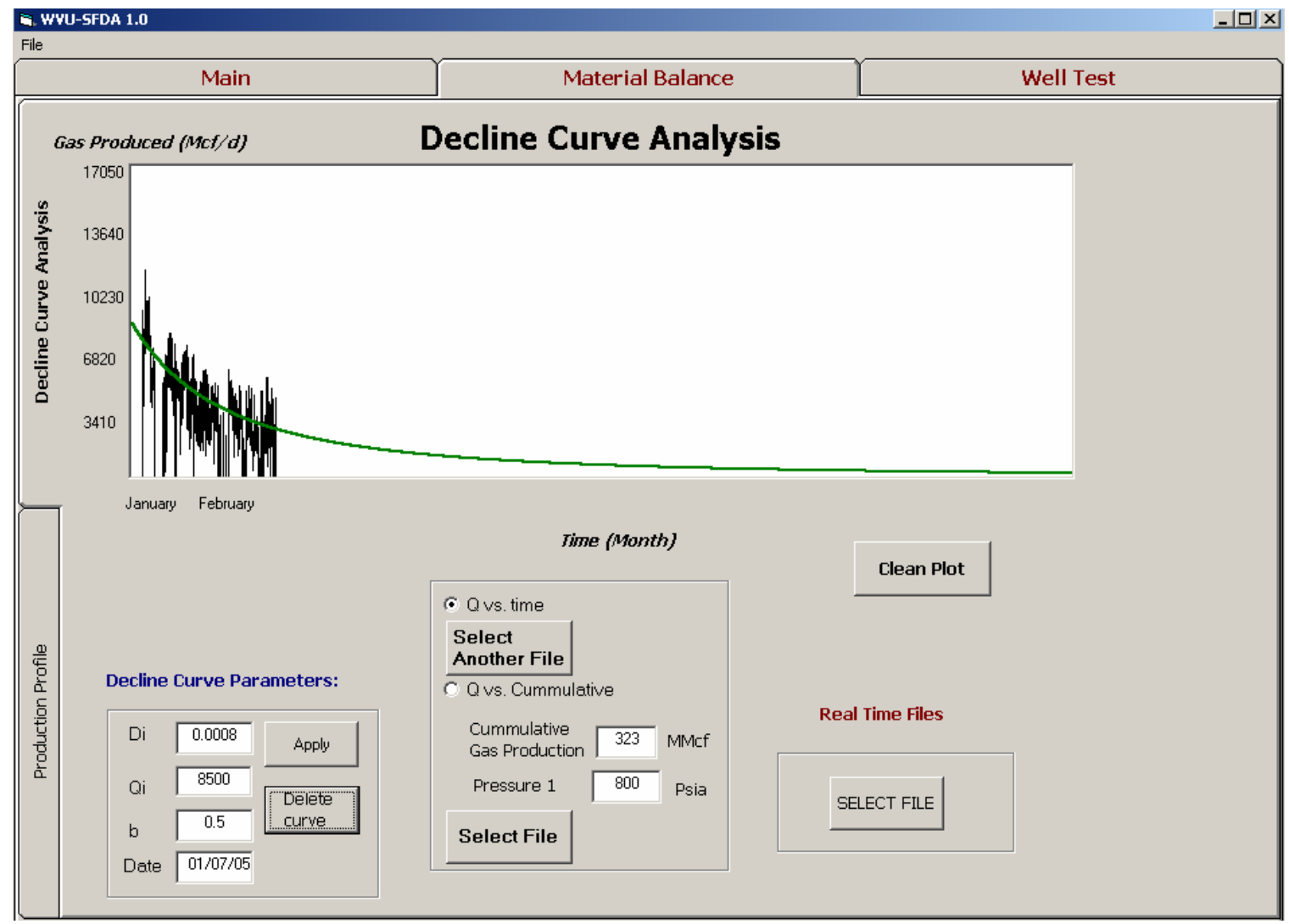

Figure 4.37- Decline Curve Analysis-Gas Produced versus time 


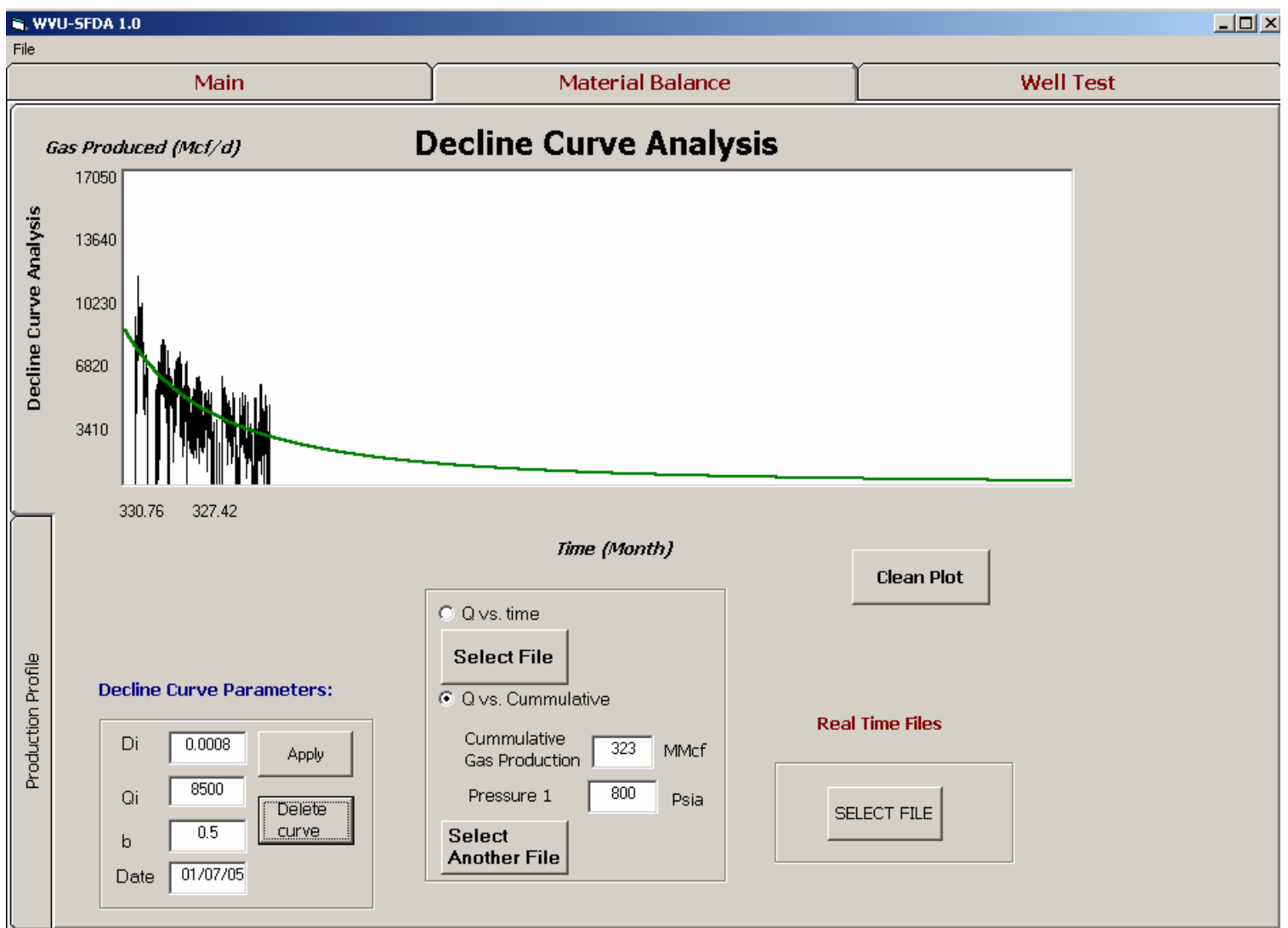

Figure 4.38- Decline Curve Analysis-Gas Produced versus cumulative production 


\section{Chapter 5}

\section{Data Control Environment-Results}

\subsection{Real Time Data-Eclipse Simulator}

Real Time Data using Eclipse Simulator was created in order to validate SFDA. Different types of scenarios were created to evaluate how SFDA handles such information and performs the reservoir analysis. In order to run the simulator, different assumptions were taken. For instance, an initial pressure of 1190 psia was selected. The well is producing at a constant flow rate of $6800 \mathrm{Mcf} /$ day. The reservoir contains one layer and the well is located in the center.

The first example shows a case where a well has been produced at a constant flow rate in which the first figure shows the result from Eclipse and the next one shows the result from SFDA.

The second example shows a case where a pressure transient period has taken place in which the results from Eclipse and SFDA are shown. Since SFDA is capable of detecting pressure build ups, the program detects the presence of the transient period, saves all the information, and makes it accessible for well testing analysis. Pseudo pressure analysis is also performed and the results are then shown.

The third example is very similar to the second one. However, in this case two pressure transient periods are present. SFDA is capable of detecting both transient periods and makes such data accessible for analysis. 


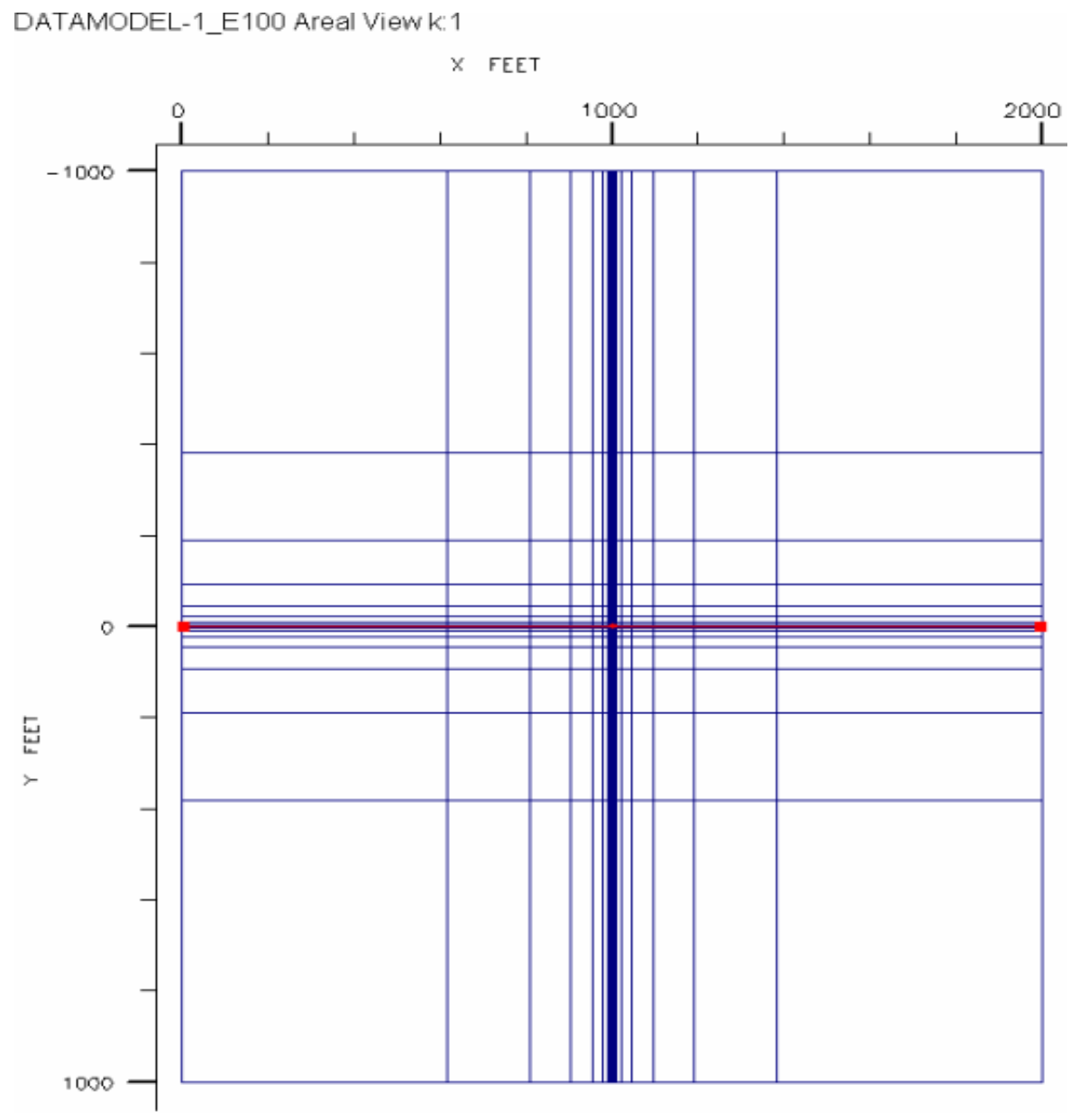

Figure 5.1-Well Location-Eclipse Result 


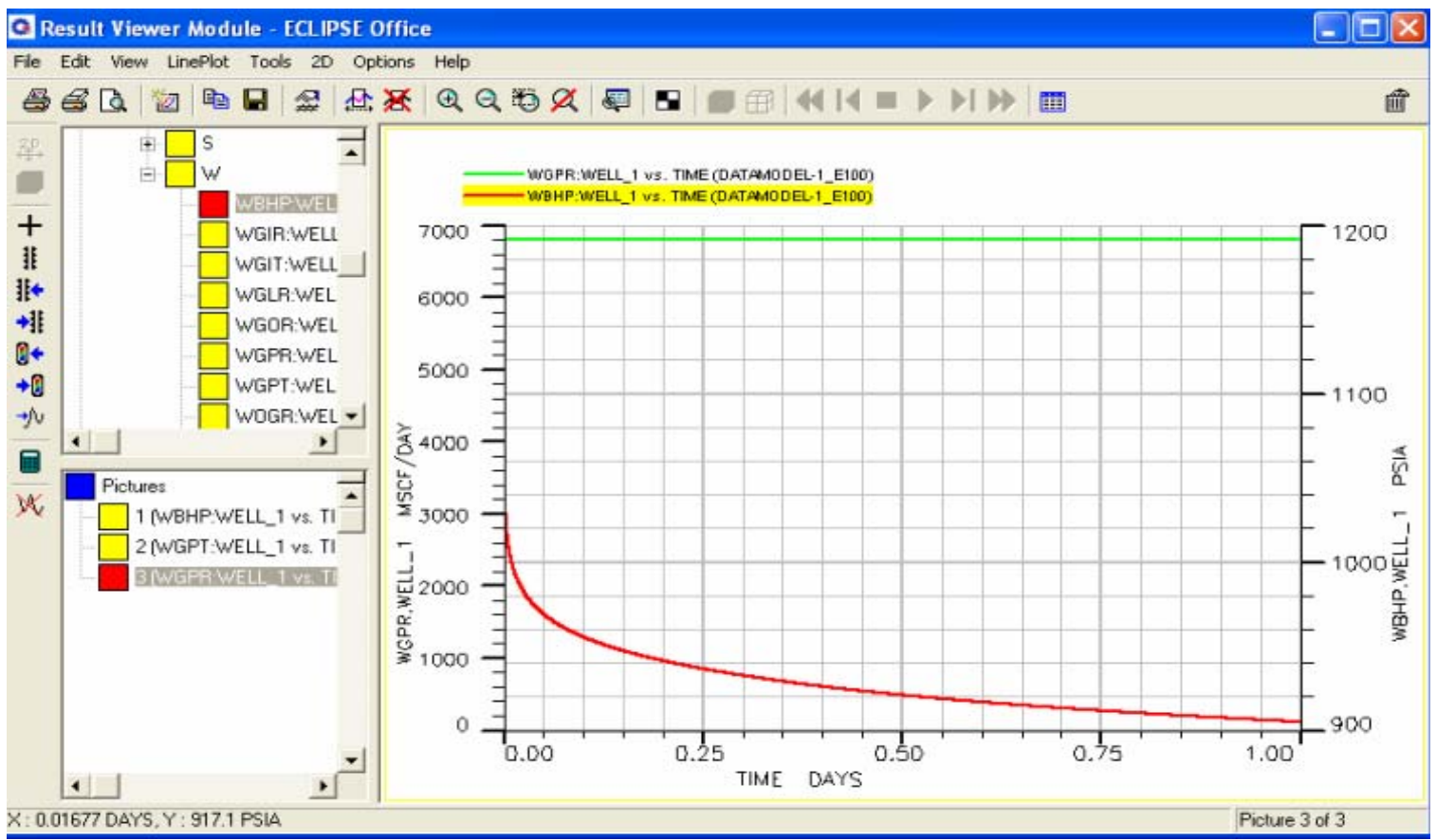

Figure 5.2-Production-Flow Rate constant- Eclipse Results

As it can be seen, SFDA shows the results of raw data and summarized data for pressure and flow rate as in the examples before (for this case, the summarized data contains less than $1 \%$ of the total values):

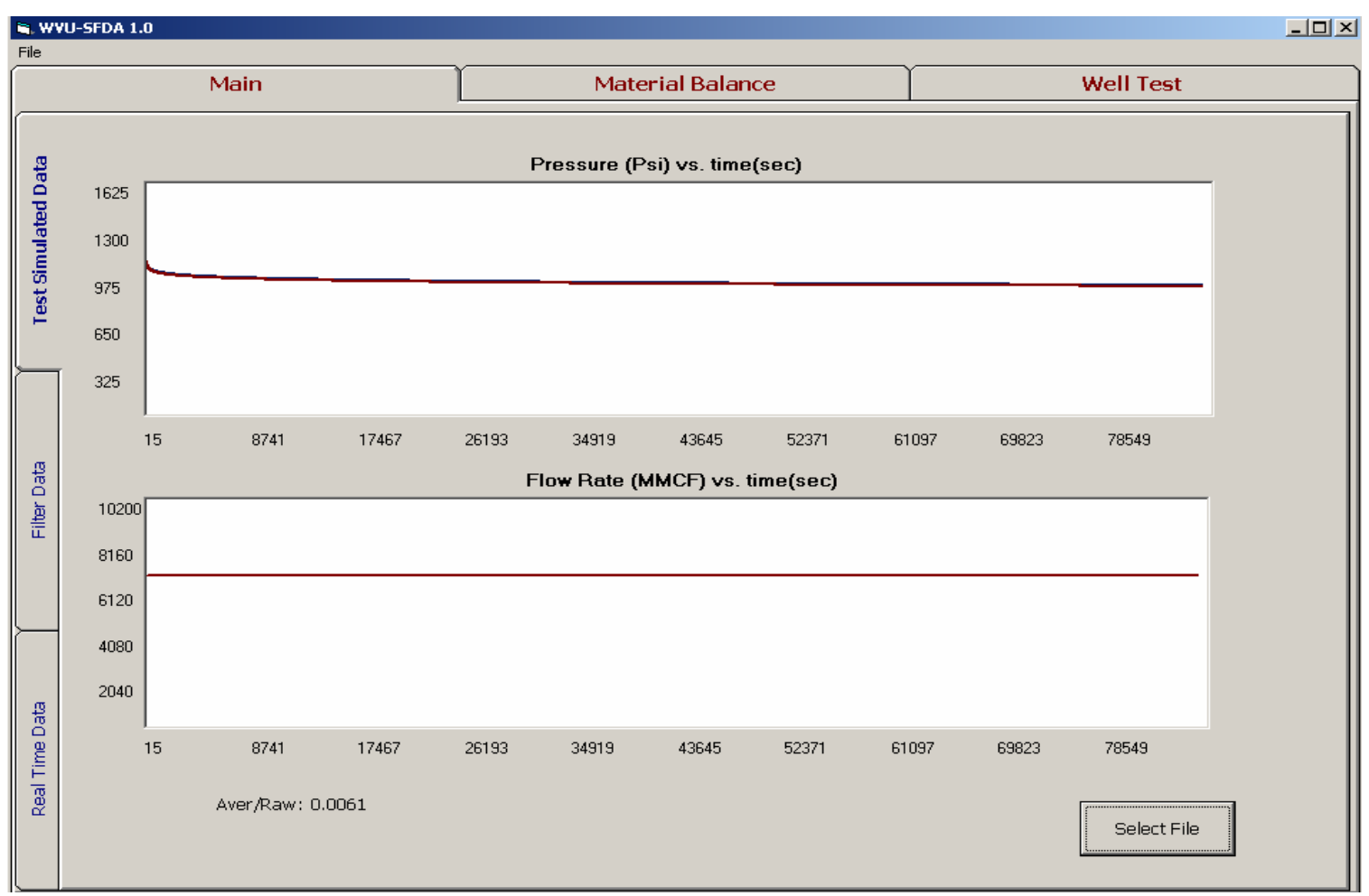

Figure 5.3-Production-Flow Rate constant-SFDA Results 
For the next case, the well has been shouted in. The next figures show the results from Eclipse and SFDA respectively. The following two figures show the results when performing well testing analysis using SFDA:

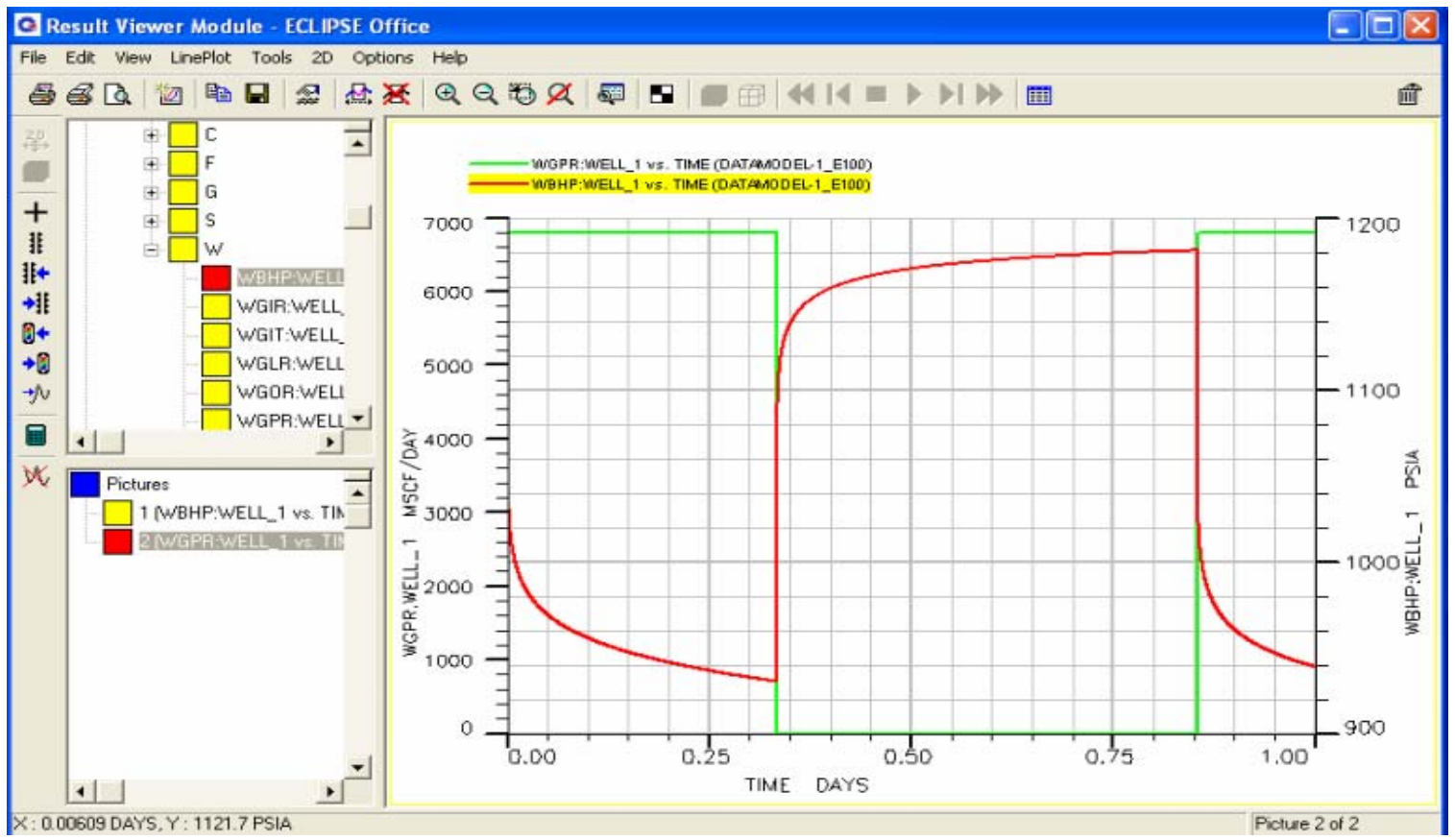

Figure 5.4-Pressure Transient Period-Eclipse Results

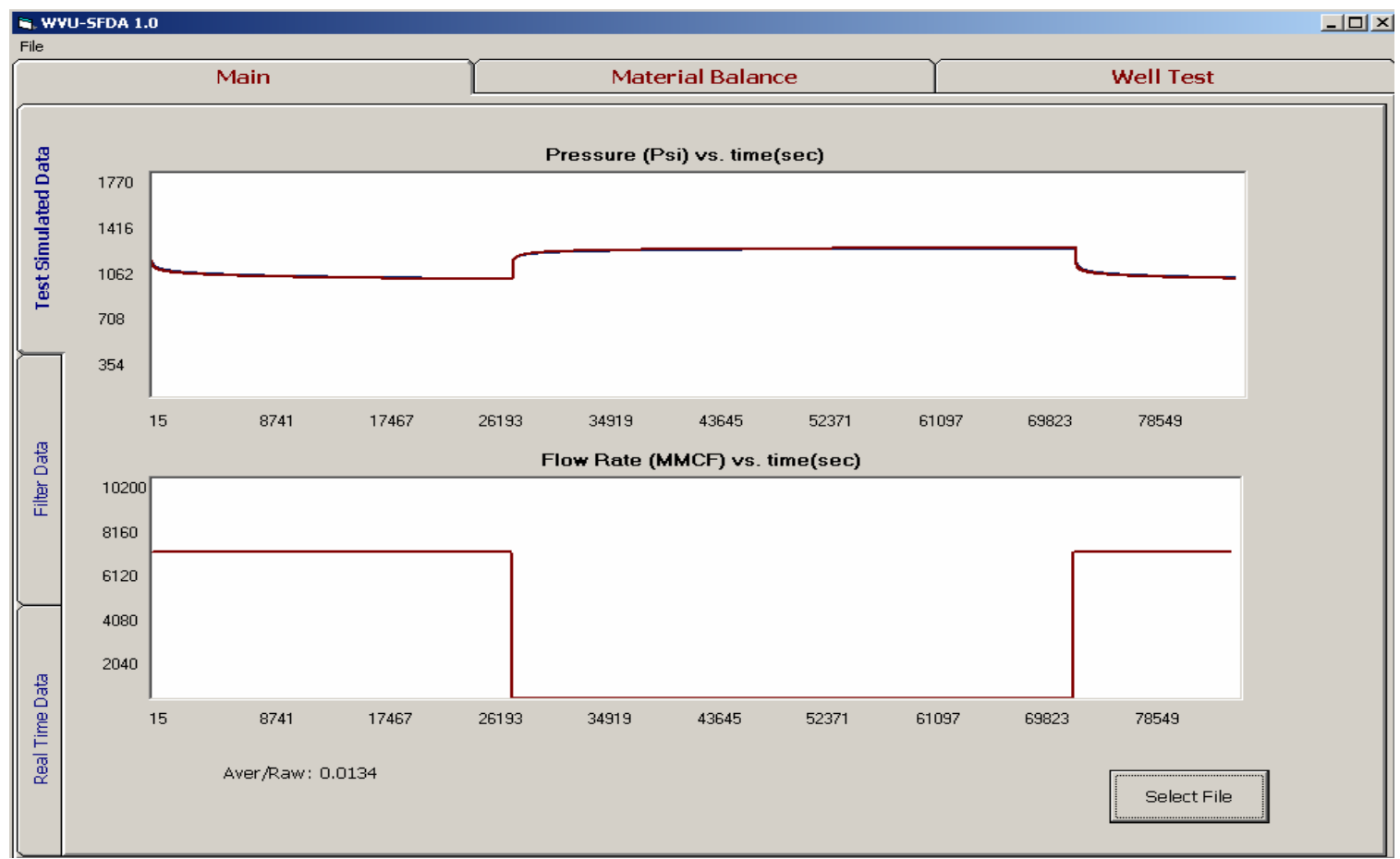

Figure 5.5-Pressure Transient Period-SFDA Results 


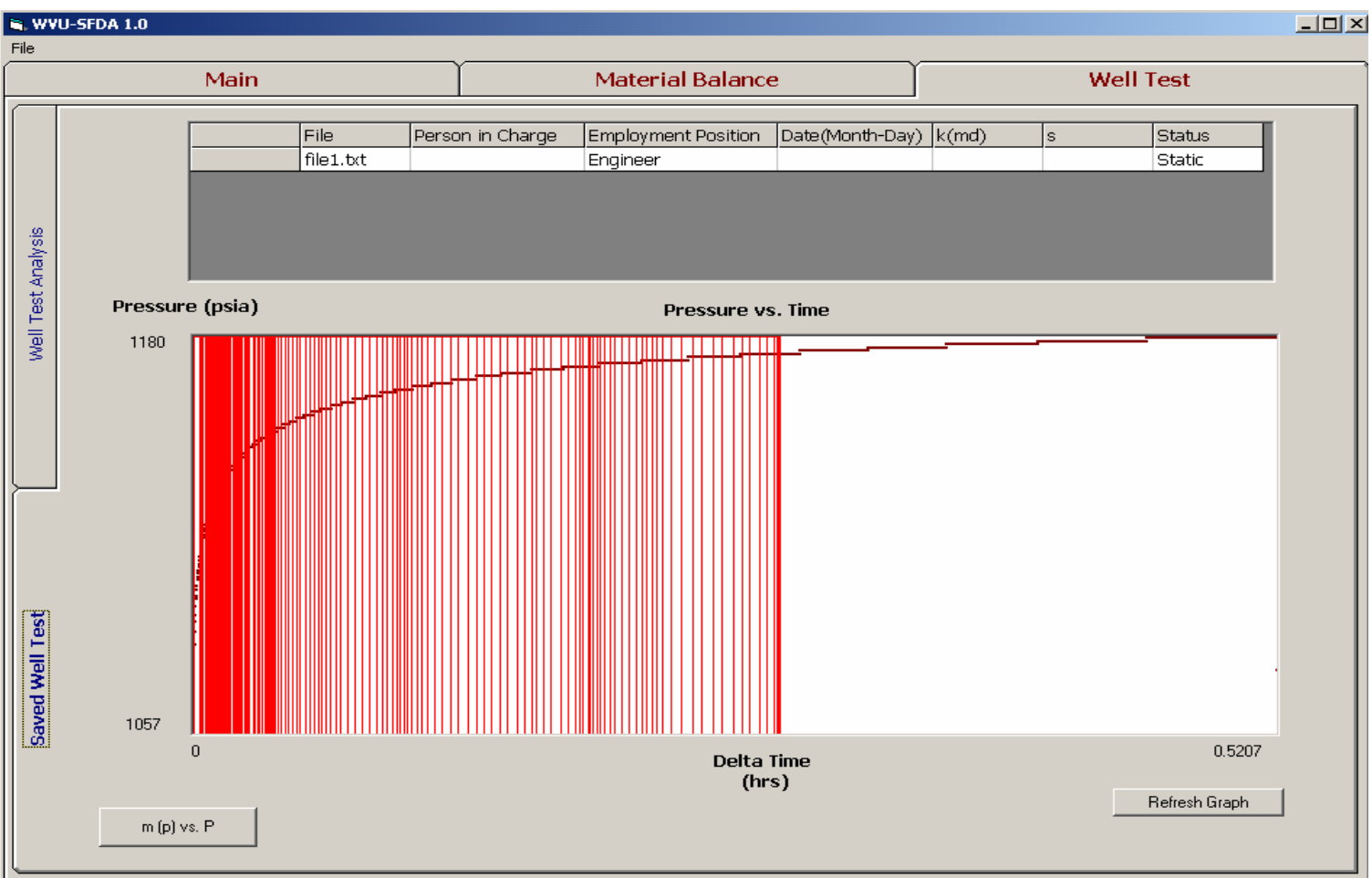

Figure 5.6- Area selection for Horner Plot

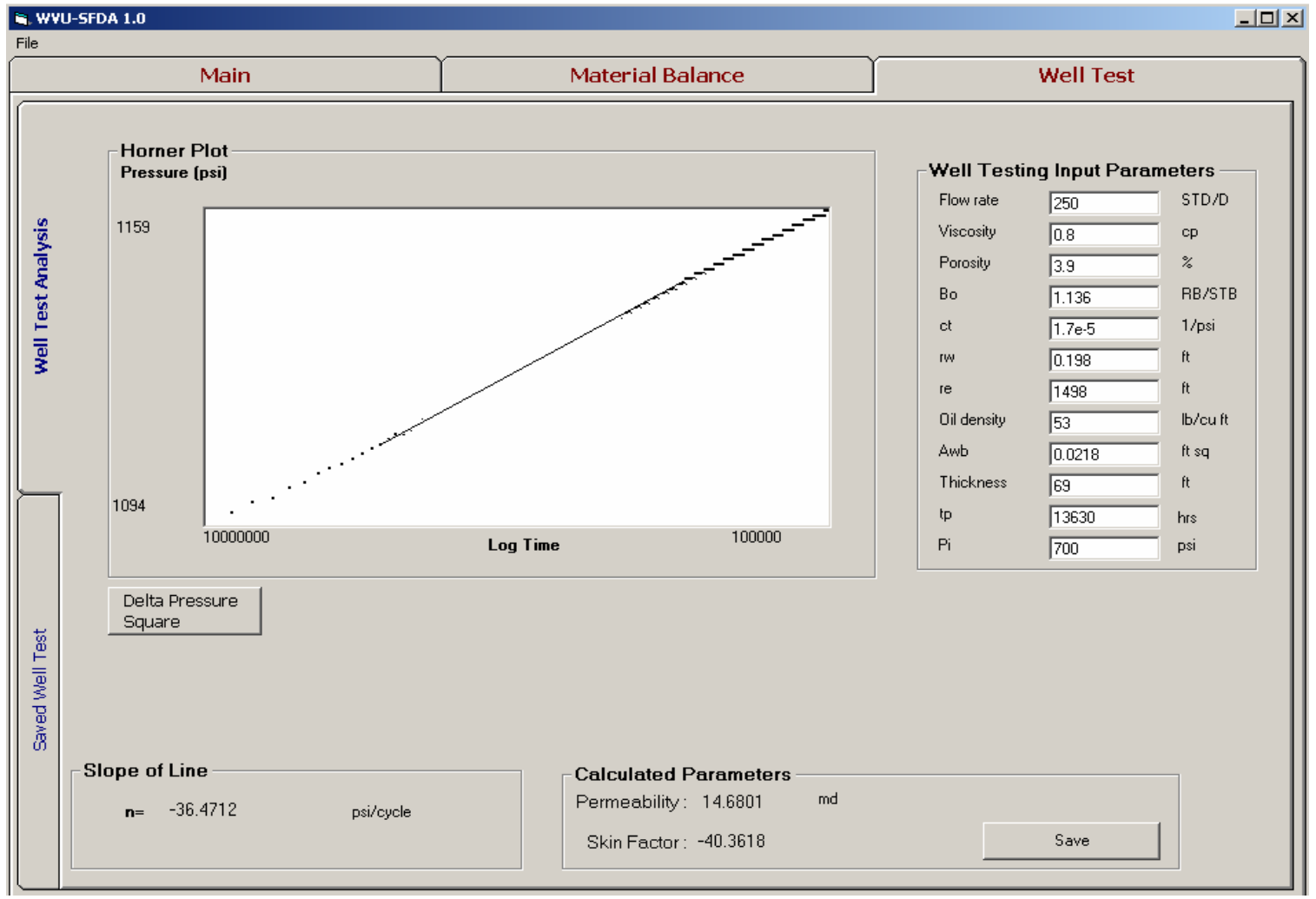

Figure 5.7- Horner Plot and Line Drawn to obtain permeability and skin factor 
The next two figures show the results after performing well testing analysis using pseudo pressure analysis. The first figure contains a plot of pseudo pressure versus pressure and the next one contains the Horner plot of pseudo pressure versus log of time:

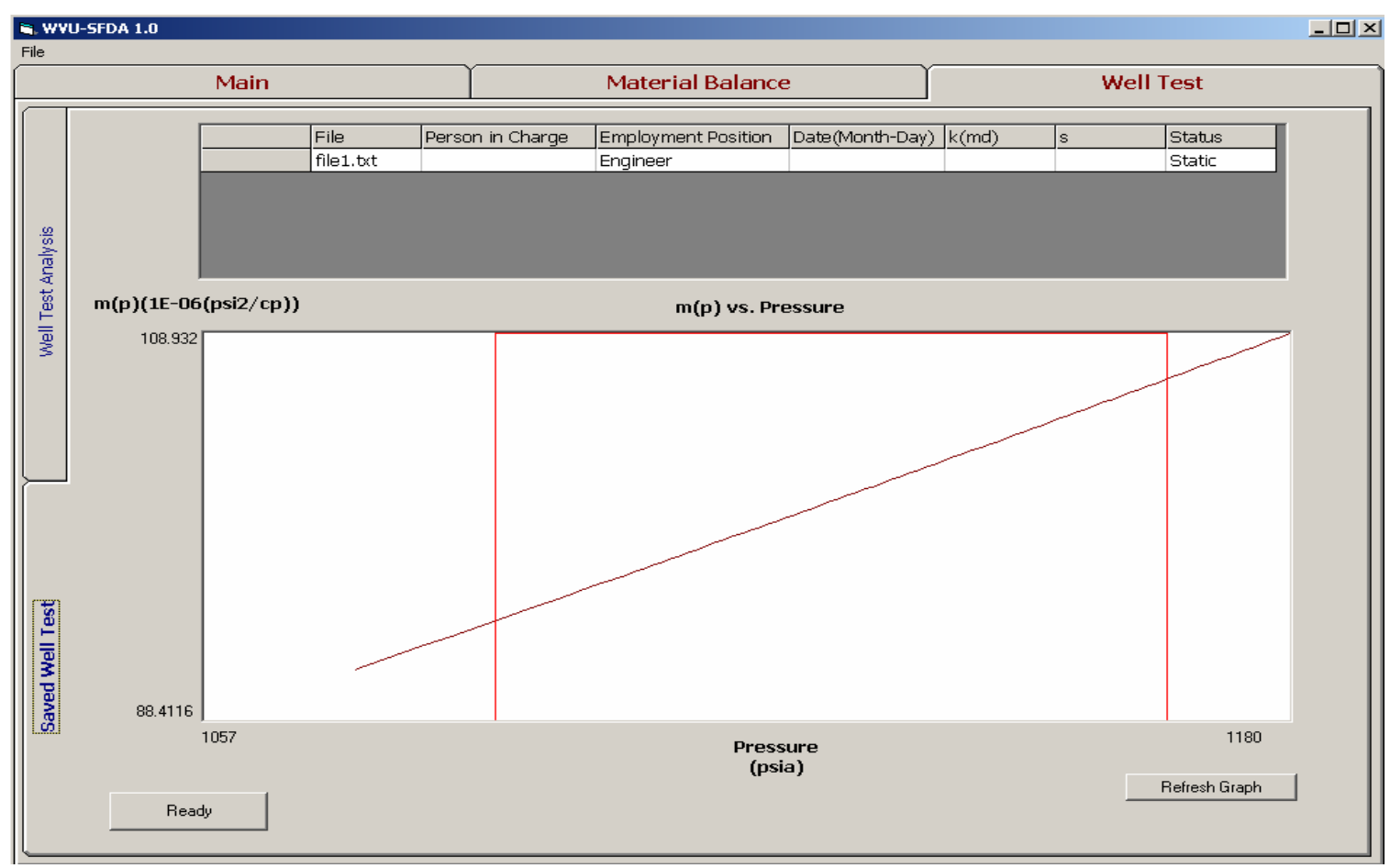

Figure 5.8-Area selection for Horner Plot-Pseudo Pressure Analysis

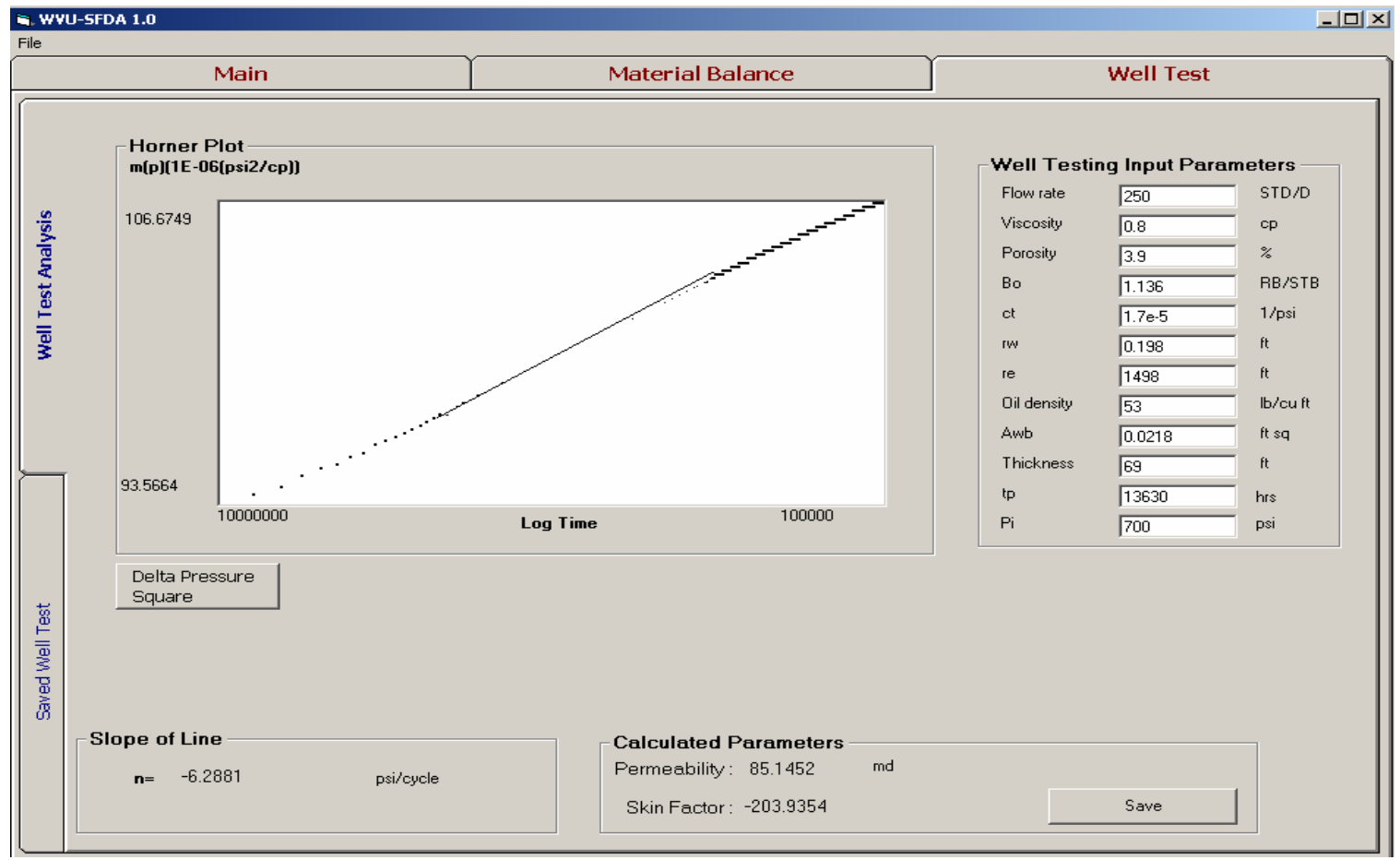

Figure 5.9- Horner Plot and Line Drawn to obtain permeability and skin factor-Pseudo Pressure Analysis 
The following example shows a case very similar to the previous one. For this case two pressure transient periods have taken place:

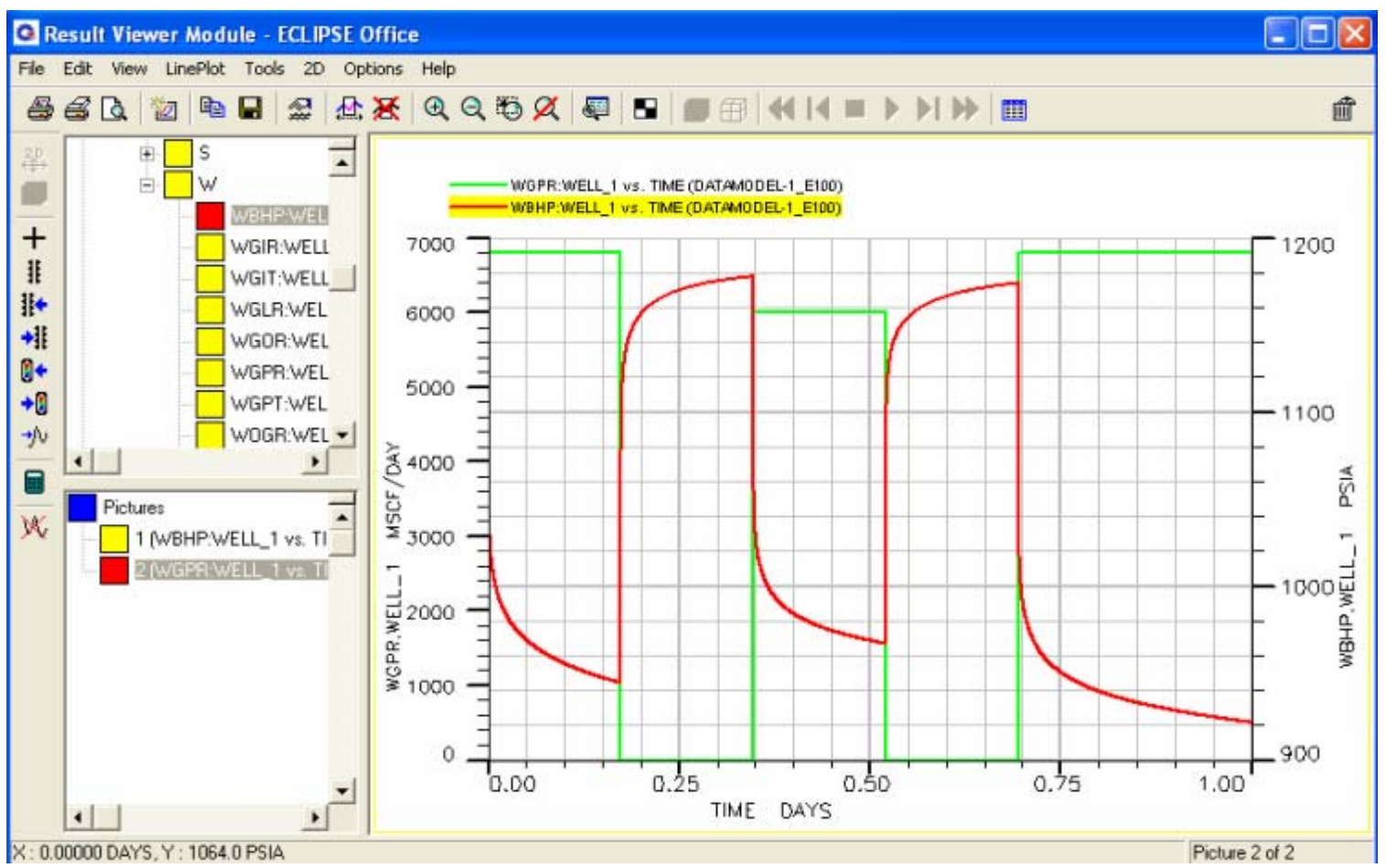

Figure 5.10- Two Pressure Transient Periods-Eclipse Results

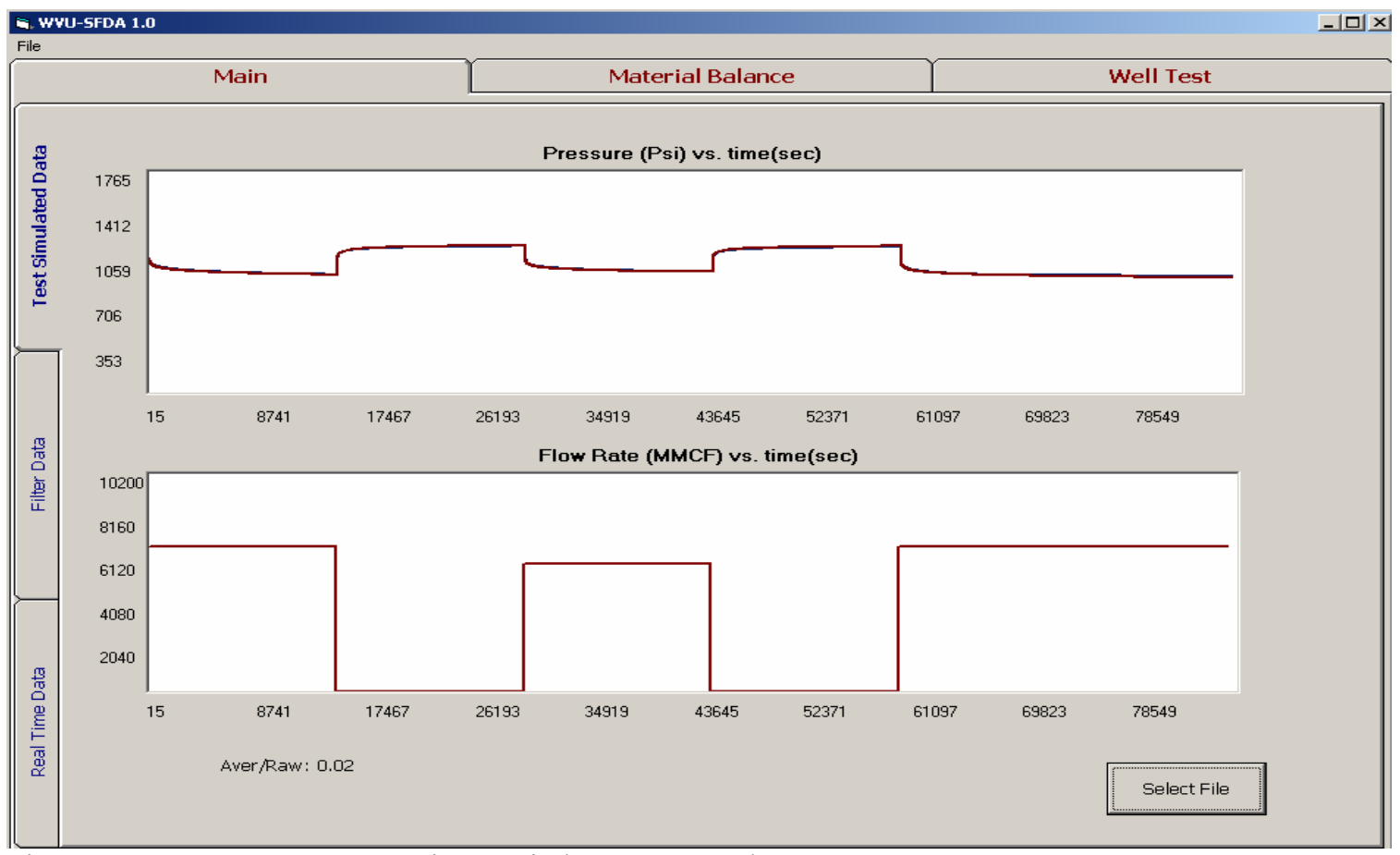

Figure 5.11- Two Pressure Transient Periods-SFDA Results 
SFDA detects both transient periods, sends all information to a file, and makes the information accessible for analysis.

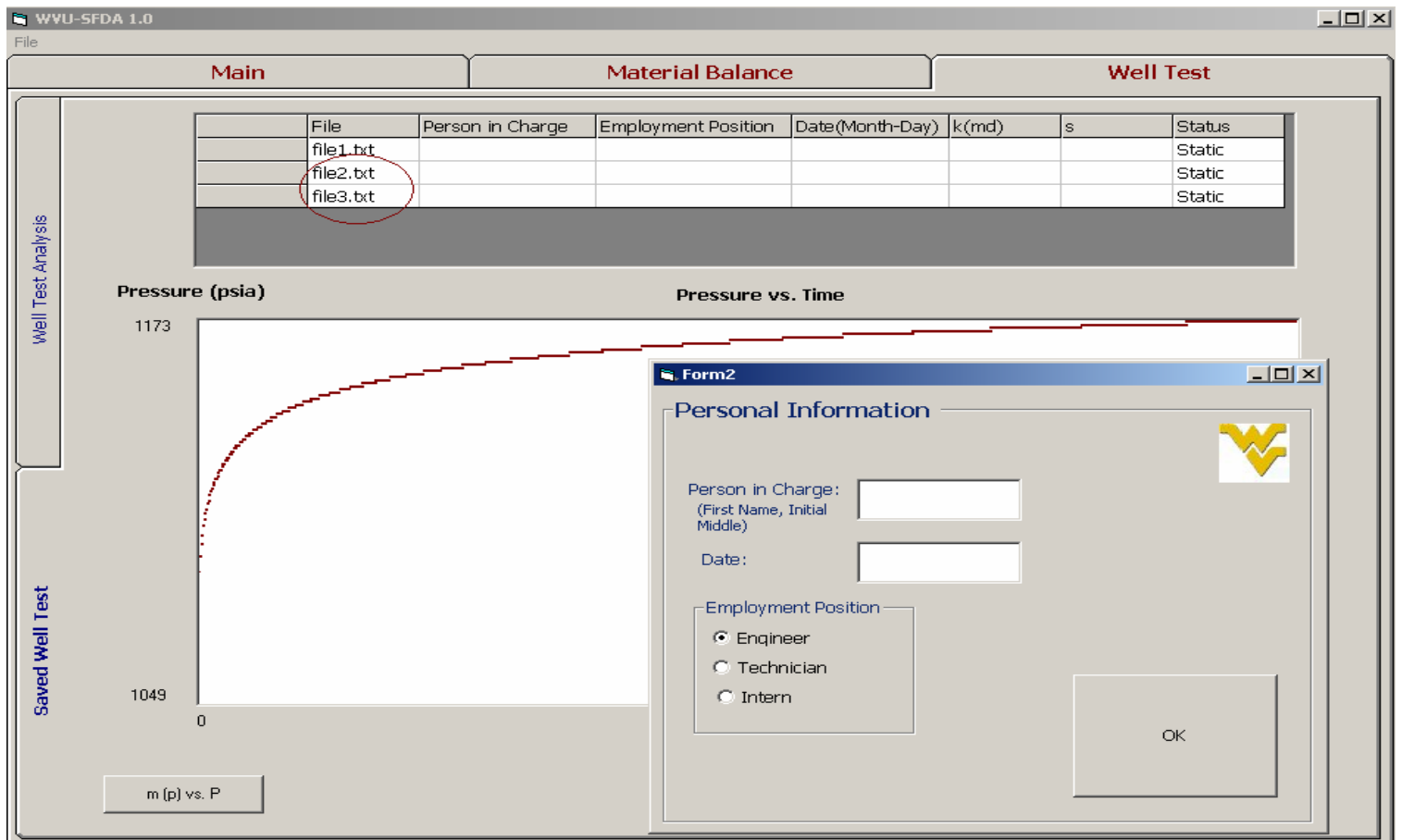

Figure 5.12- Area selection for Horner Plot-Pseudo Pressure Analysis

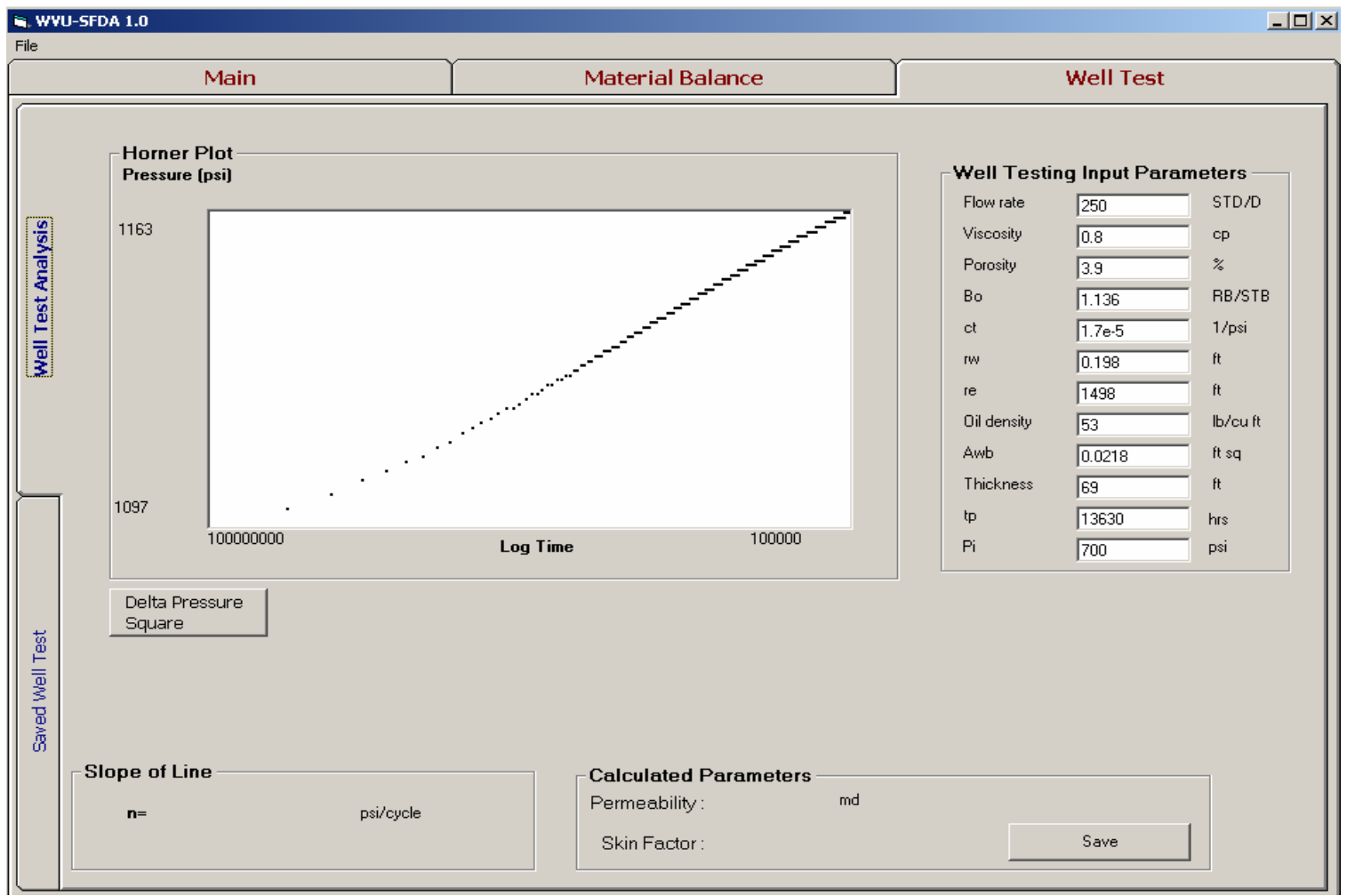

Figure 5.13- Horner Plot and Line Drawn to obtain permeability and skin factor 


\section{Chapter 6}

\section{Concluding Remarks}

The first building block for Smart Field Data Analysis has been created and the problems encountered throughout its development have been solved analytically. The goal for developing SFDA has been primarily to handle and summarize the large volume of data from the real time measurements and to create profiles for pressure, flow rate, and gas in place using sets of summarized data. Pressure transient analysis in real time has been included as one of the reservoir analyses the software is capable of performing in which the presence of a transient period can be detected as the high frequency data is being monitored. Such information is then saved and prepared to perform well test analysis. A new approach to create value once the high frequency is summarized has been developed in which gas in place is monitored to determine whether a leak is presented in the reservoir in order to alert the engineers and react in a timely manner.

Decline Curve Analysis has been carried out as well in order to forecast production performances.

The entire process has been executed using real time from an actual field provided by Columbia Gas Transmission Corporation and real time generated from Eclipse Simulator to corroborate that the software is capable of working on different scenarios 


\subsection{Conclusions}

Considering the results presented in chapter 4 and 5, different conclusions have been made:

1. High frequency data can be aggregated using an average technique that summarizes the actual data while preserving its integrity.

1.1 The average technique can discard at least $70 \%$ of the actual data and no more than $30 \%$ can be used for analysis

2. Pressure transient period can be detected in real time and the high frequency data can be used to perform well testing analysis and obtain permeability and skin factor for several periods.

2.1 Pseudo pressure analysis can also be performed and values for permeability and skin factor can be obtained from such an analysis

3. In order to create a value from the real time data once it has been summarized, material balance has been performed in which the gas in place is estimated at different times to determine whether there is an existing leak in the reservoir.

4. Decline Curve analysis can also be performed in which the summarized data can be used to forecast future production behavior.

5. The program has been tested using both real time data generated from actual production fields provided by Columbia Gas Transmission Corporation and real time generated from Eclipse Simulator to demonstrate that the methodology used for this investigation works for different case scenarios. 


\subsection{Future Work}

The methodology presented throughout this investigation can be used as the basis for building a more complex computer model capable of performing different types of production analysis in real time, predicting production forecast, and alerting the engineers for abnormal situations that will be encountered during production operations. The aim of developing such a simulator is to minimize the time of reaction to particular events and optimize production.

Different sections from SFDA can be very useful when building a more complex computer program. The average technique that summarizes the data to a handful amount as data is being monitored in real time, the process of detecting pressure transient periods in real time, as well as the process of monitoring gas in place continuously, represent the basis blocks for building such a complex computer program.

Entirely new possibilities related to Smart Field Data Analysis have been presented and further analysis can be done in order to make the production processes more efficient and effective. 


\section{Bibliography}

[1] Oberwinkler, C., and Stundner, M. (2005): 'From Real-Time Data to Production Optimization', paper SPE 87008, 2004 SPE Asia Pacific Conference, March 29-30, Kuala Lumpur, Malaysia.

[2] Oberwinkler, C., Holland J., Mayfield D., and Dixon D (2005): 'Real-Time Information to the Engineer's Desktop-How Murphy E\&P Improved Production Management', paper SPE 96645, 2005 SPE Program Committee, September 6-9, Scotland, U.K.

[3] Saputelli, L., Economides, M., Nikolaou, M. and Kelessidis, V (2003): 'Real-Time Decision-making for Value Creation while Drilling', paper SPE 85314, 2003 SPE/IADC Middle East Drilling Technology Conference \& Exhibition, October 20-22, Abu Dhabi, UAE.

[4] Saputelli, L., Mochizuki, S., Hutchings, L., Cramer, R., Anderson, M.B, Mueller, J.B., Escorcia, A., Harms, A.L., Sisk, Pennebaker, S., Han, J.T., Brown, A., Kabir, C.S., Reese, R.D., Nuñez, G.J., Landgren, K.M., Mckie, C.J., and Airlie, C (2003): 'Promoting Real-Time Optimization of Hydrocarbon Producing Systems', paper 83978, 2003 SPE Program Committee, September 2-5, Aberdeen, U.K.

[5] Stundner, M. and Al-Thuwaini, J.S (2001): 'How Data-Driven Modeling Methods like Neural Networks can help to integrate different Types of Data into Reservoir Management', paper SPE 68163, 2001 SPE Middle East Oil Show, March 17-20, Bahrain.

[6] Saputelli, L., Nikolaou, M., and Economides, M.J (2005): 'Self-Learning Reservoir Management', paper SPE 84064, 2005 SPE Annual Technical Conference and Exhibition, October 5-8, Denver, Colorado, USA.

[7] Mochizuki, S., Saputelli, L.A., Kabir, C.S., Cramer, R., Lochmann, M.J., Reese, R.D., Harms, L.K., Sisk, C.D., Hite, J.R., Escorcia, A (2004): 'Real Time Optimization: Classification and Assessment', paper SPE 90213, 2004 SPE Annual Technical Conference and Exhibition, September 26-29, Houston, Texas, USA.

[8] Holland, J., Oberwinkler, C., Huber, M. and Zangl, G (2004): 'Utilizing the Value of Continuously Measured Data', paper SPE 90404, 2004 SPE Annual Conference and Exhibition, September 26-29, Houston, Texas, USA. 
[9] Stundner, M., De Jonge, G., and Zangl, G (2003): 'Automated Reservoir Surveillance through Data Mining Software', paper SPE 83974, 2003 SPE Program Committee, September 2-5, Aberdeen, U.K.

[10] Athichanagorn, S., Horne, R.N., and Kitani, J (1999): 'Processing and Interpretation of Long-term Data from Permanent Downhole Pressure Gauges', paper SPE 56419, 1999 SPE Annual Conference and Exhibition, October 3-6, Houston, TX, USA.

[11] Coludrovich E.J., McFadden, J.D., Palke, M.R., Roberts, W.R., and Robson, L.J (2004): 'The Boris Field Well Management Philosophy-The Application of Permanent Downhole Flowmeters to Pressure Transient Analysis: An Integrated Approach', paper SPE 90316, 2004 SPE Annual Technical Conference Exhibition, September 26-29, Houston, Texas, USA.

[12] Der Kinderen, W.J.G.J, Dunham, C.L., and Poulisse, H.N.J (1998): 'Real-Time Artificial Lift Optimization', paper SPE 49463, 1998 Abu Dhabi International Petroleum Exhibition and Conference, October 11-14, Abu Dhabi, UAE.

[13] De Jonge, G. and Stundner M (2002): 'How Routine Reservoir Surveillance with Neural Networks and Simplified Reservoir Models can Convert Data into Information', paper SPE 78334, $200213^{\text {th }}$ European Petroleum Conference, October 29-31, Aberdeen, Scotland.

[14] Mora, O., Startzman, R.A. and Saputelli, L (2005) 'Maximizing Net Present Value', paper SPE 94664, 2005 SPE Hydrocarbon Economics and Evaluation Symposium, 2005 April 3-5, Dallas, Texas, USA.

[15] Dunham, C.L (2003) 'Production Automation in the $21^{\text {st }}$ Century: Opportunities for Production Optimization and Remote Unattended Operations', paper SPE 79390, 2003.

[16] Athichanagorn, S., and Horne, R.N (1995) 'Automatic Parameter Estimation from Well Test Data Using Artificial Neural Network', paper SPE 30556, 1995 SPE Annual Technical Conference and Exhibition, 1995 October 22-25, Dallas, Texas, USA.

[17] Jacobsen J.S., Bethel, E.W., Datta-Gupta, A., and Holland, P.J (1995) 'Petroleum Reservoir Simulation in a Virtual Environment', paper SPE 29118, $13^{\text {th }}$ SPE Symposium on Reservoir Simulation, February 12-15, San Antonio, Texas, USA.

[18] Omotosho, R.J (2004): 'Permanent Downhole Equipments Sensors in Today's Petroleum Industry', M.Sc. Thesis, The University of Texas at Austin, Austin, Texas, USA. 
Appendix 


\section{Smart Field Data Analysis Software (SFDA 1.0)}

\section{User Manual}

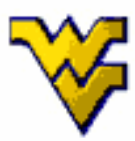

West Virginia University

2007 
This document will initiate you to the latest software in Smart Field Data Analysis in which high frequency data can be uploaded and the changes in pressure and flow rate can be observed in Real-Time. In addition, the software will be capable of handling and summarizing the enormous amount of data and carry out certain production analysis such as material balance and decline curve analysis. The computer program will also detect pressure transient periods in which the information related to such periods will be saved and prepared for the user to perform well test analysis.

The program consists on three main tabs: Main, Material Balance, and Well Test. The Main tab will allow you to create a project, open it, select the real-time data and upload it to see changes in pressure and flow rate in real time, check for the difference between summarized data and actual one, change preset values, upload data generated by ECLIPSE and see how the summarized data differs from the actual one. The Material Balance Tab will allow you to observe how the gas in place changes in real time, upload summarized monthly production data to see pressure, flow rate, and gas in place profiles, and use the summarized monthly data to perform decline curve analysis. The Well Test Tab will show all the pressure transient period detected by SFDA in which you can easily select those files to perform well test analysis. You will be able to select an area and move to another window to see the Horner Plot and draw a line to obtain permeability and skin factor.

The name of the Software is SFDA-Smart Field Data Analysis-The figure shown below will be the interface you will see when opening SFDA.

As you see, the three tabs in red shown horizontally are the main tabs. Each one of those tabs has sub-tabs which are shown vertically in blue.

The first step will be to click on the File button: 


\section{MAI N Tab:}

On this section, you will be able to open a file and see how pressure and flow rate change with time as data feeds the system in real-time. You will use this section to observe the behavior of pressure and flow rate in real time.

When the program starts reading data in real time, SFDA filters the data and creates sets of summarized data. The program will be detecting pressure transient periods in which such information will be saved. At the same time SFDA will identify and remove outliers. "Everything will be done in real-time".

The Main Tab will have the following sub-tabs: Real Time Data, Filter Data, and Test Simulated Data. The Real Time Data will allow you to observe the changes in pressure and flow rate in real time. The Filter Data Tab will allow you to see how the summarized data differs from the actual one after a day has passed. The Test Simulated Data will allow you to upload daily data from ECLIPSE and see again how the summarized data differs from the actual one.

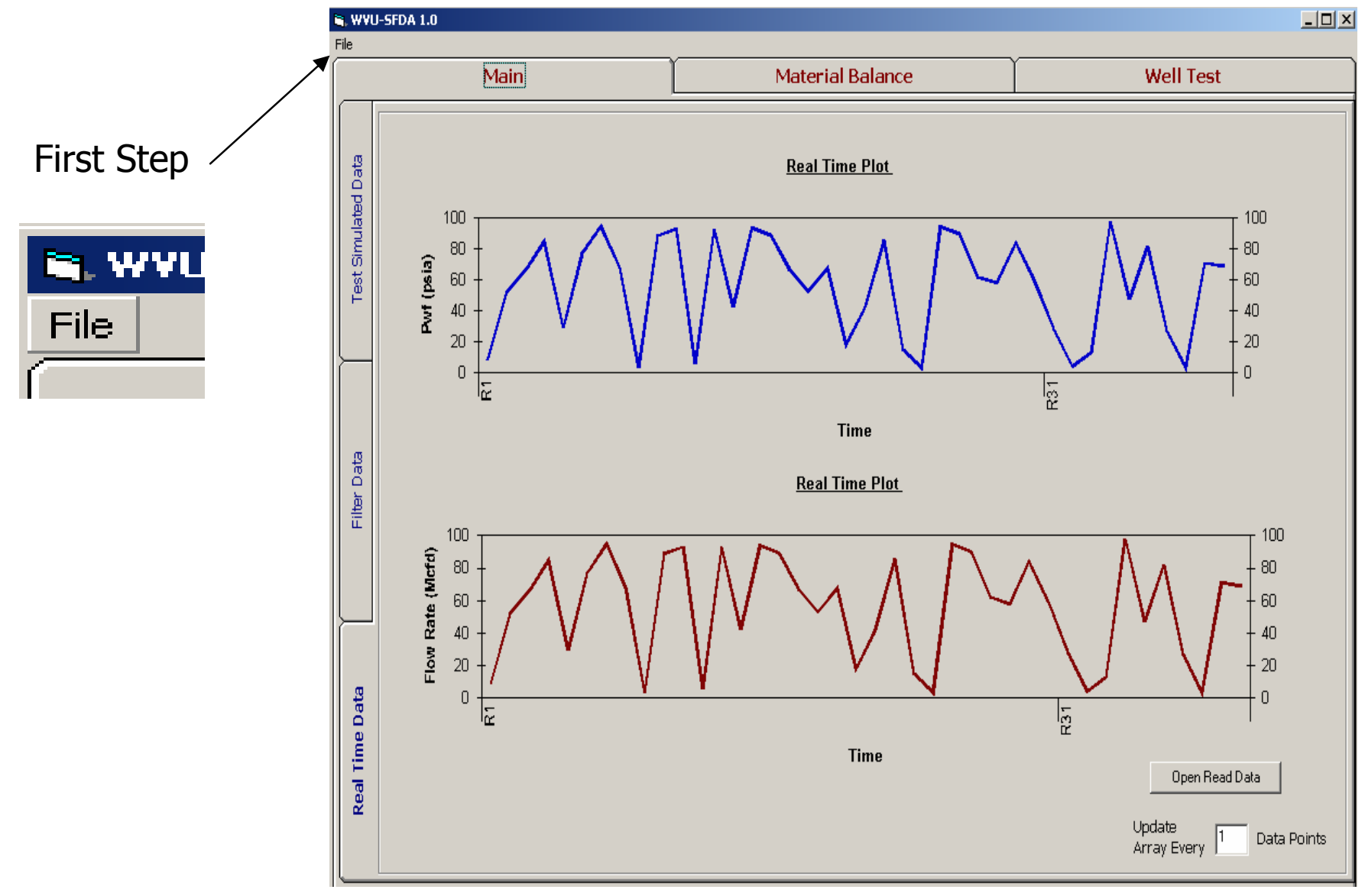




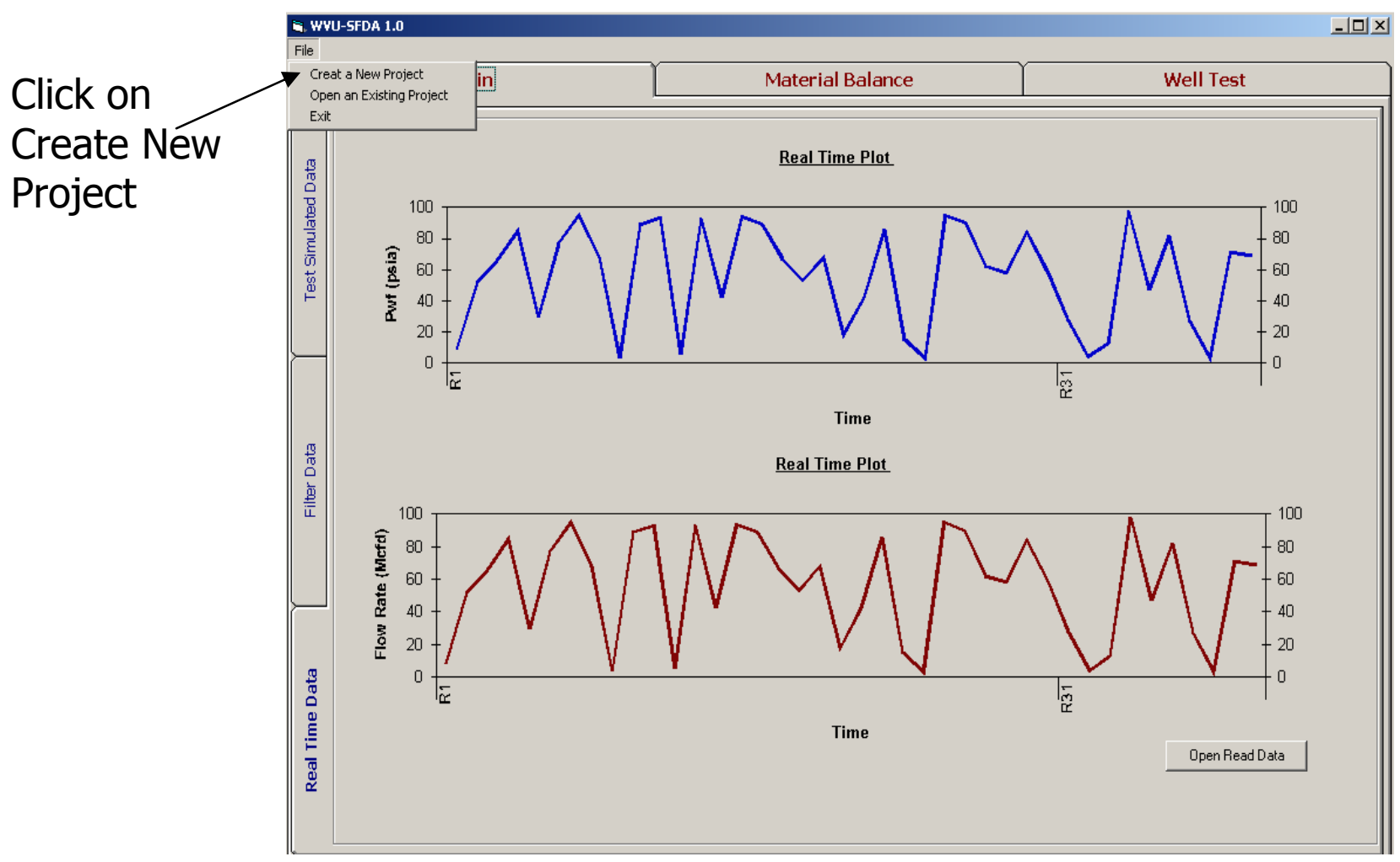

\section{E. MHU-SFDA 1.0}

\section{File}

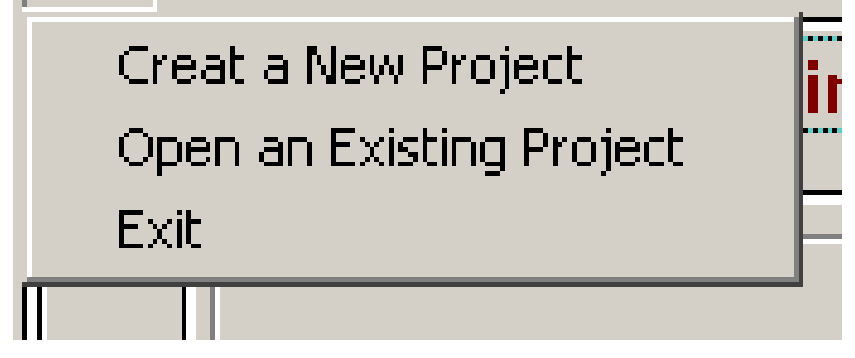


You should find the folder where the data is located and create a project, name it and then click on the save button.

\section{Select a Location}

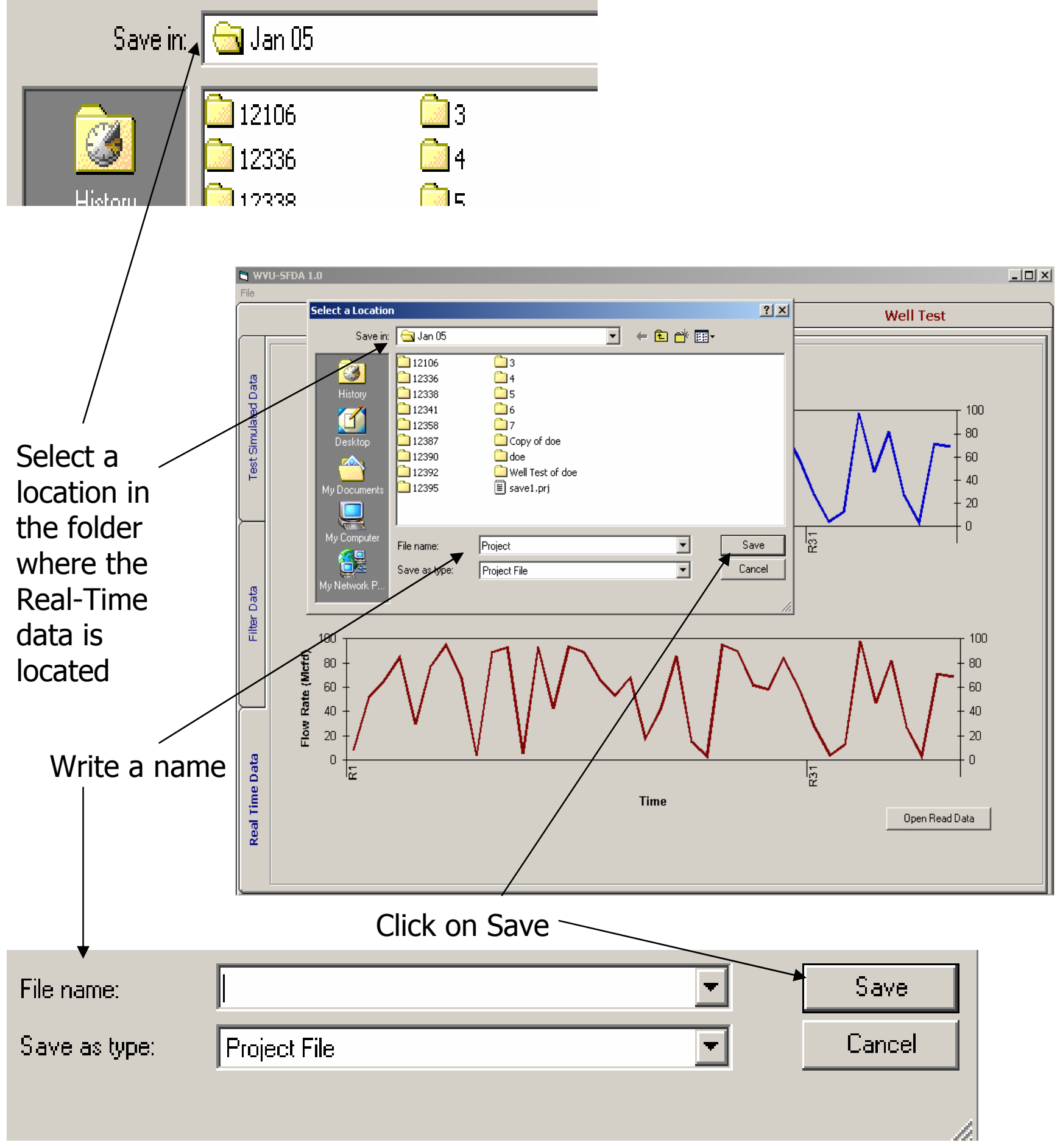


The same window will appear in which you will click on "Open" in order to open the project already created.

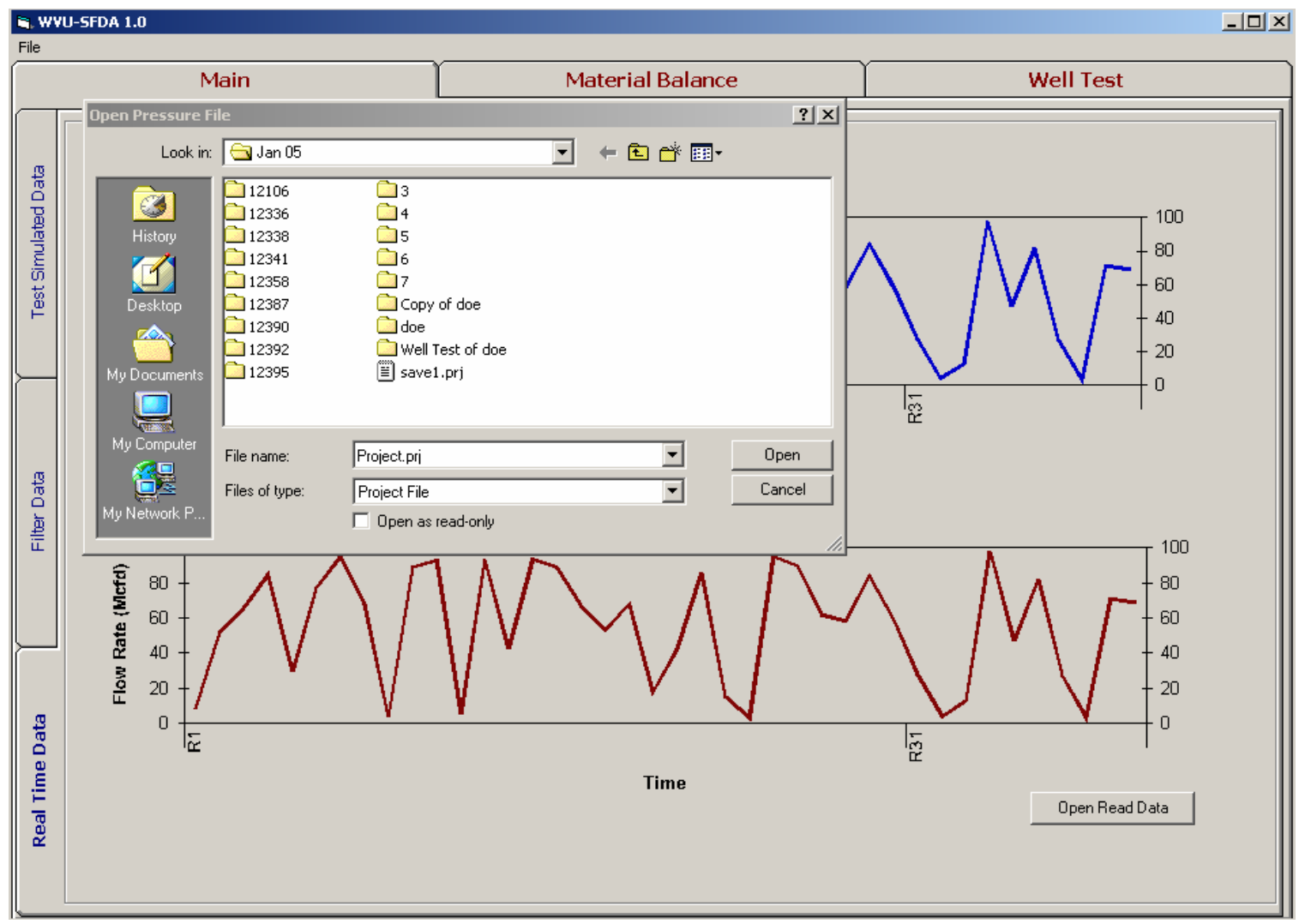

\begin{tabular}{lll|} 
File name: & Project.pi & Open \\
\cline { 2 - 3 } & Files of type: & Project File \\
& $\Gamma$ Open as read-only & \\
& &
\end{tabular}


If you decide to open a project already created, at the beginning just click on "Open an Existing Project" and then the "Open" button.

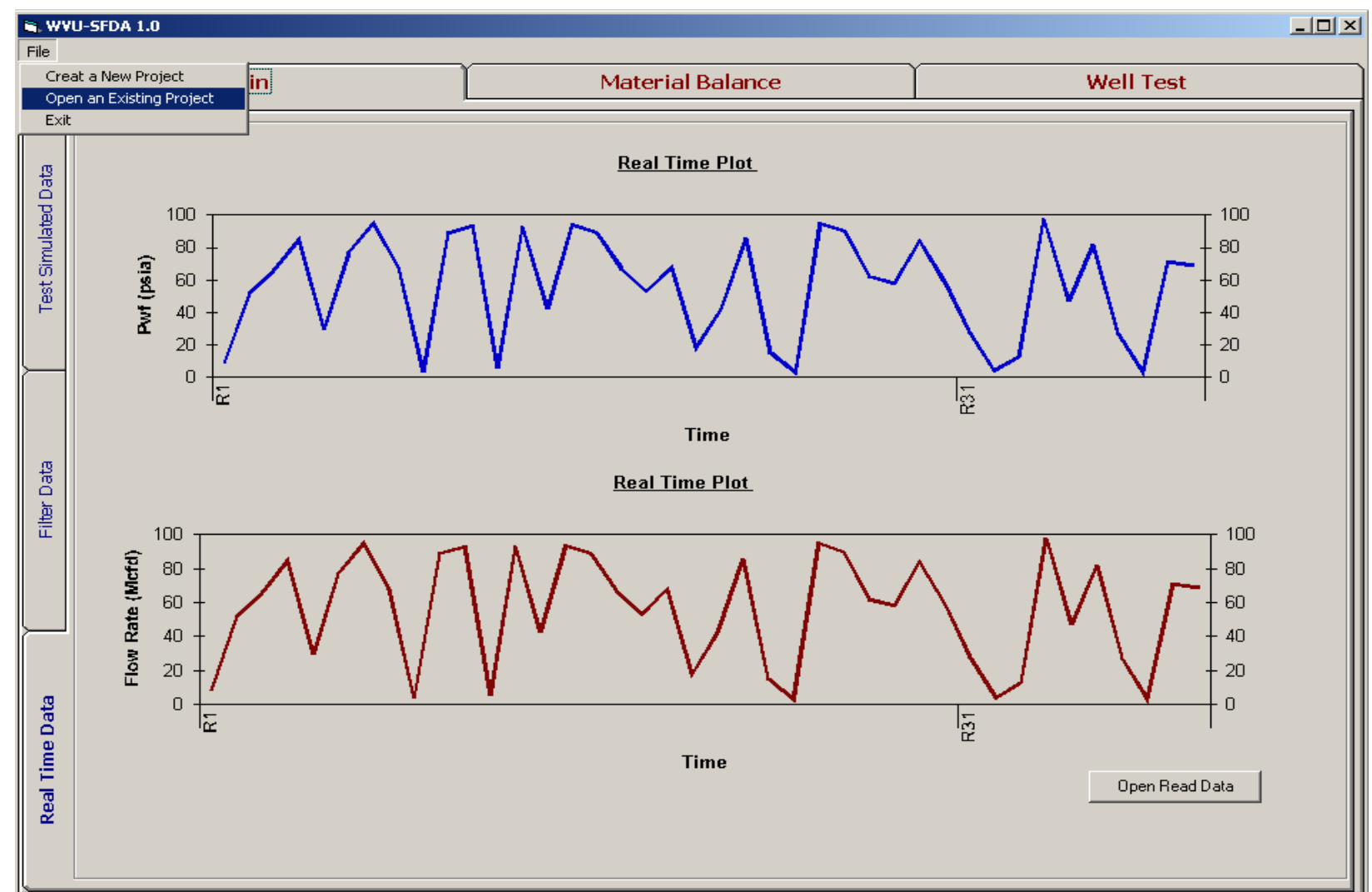

\section{E= HMU-SFDA 1.D}

\section{File}

Creat a New Project

Ореп вп Ехіsting Projert

Exit 


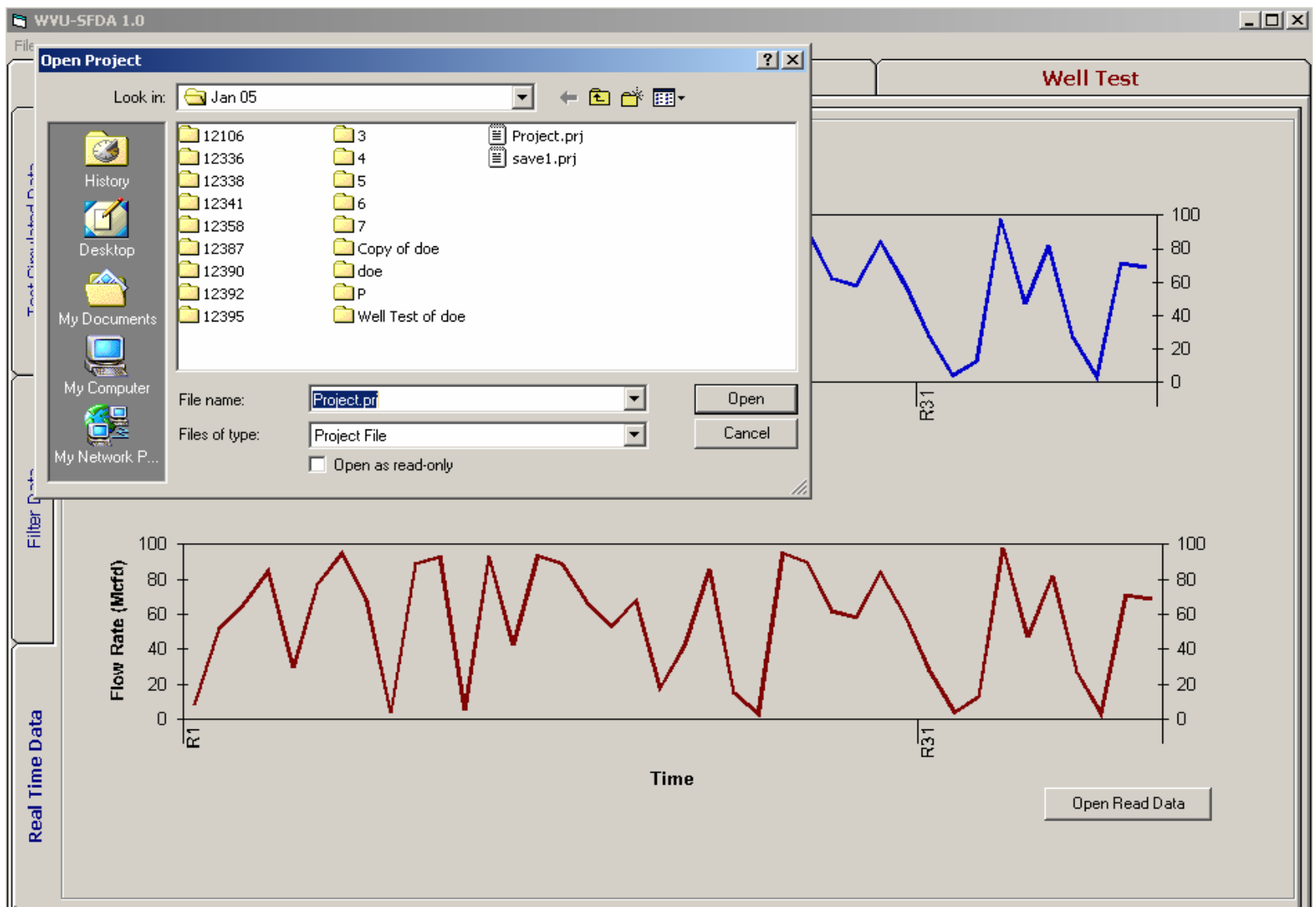

\begin{tabular}{|c|c|c|c|}
\hline File name: & Project.pr & - & Open \\
\hline Files of type: & Project File & 7 & Cancel \\
\hline
\end{tabular}


Once the project already created has been opened; you will click again on the File Selection the option of "Open an Existing Project".

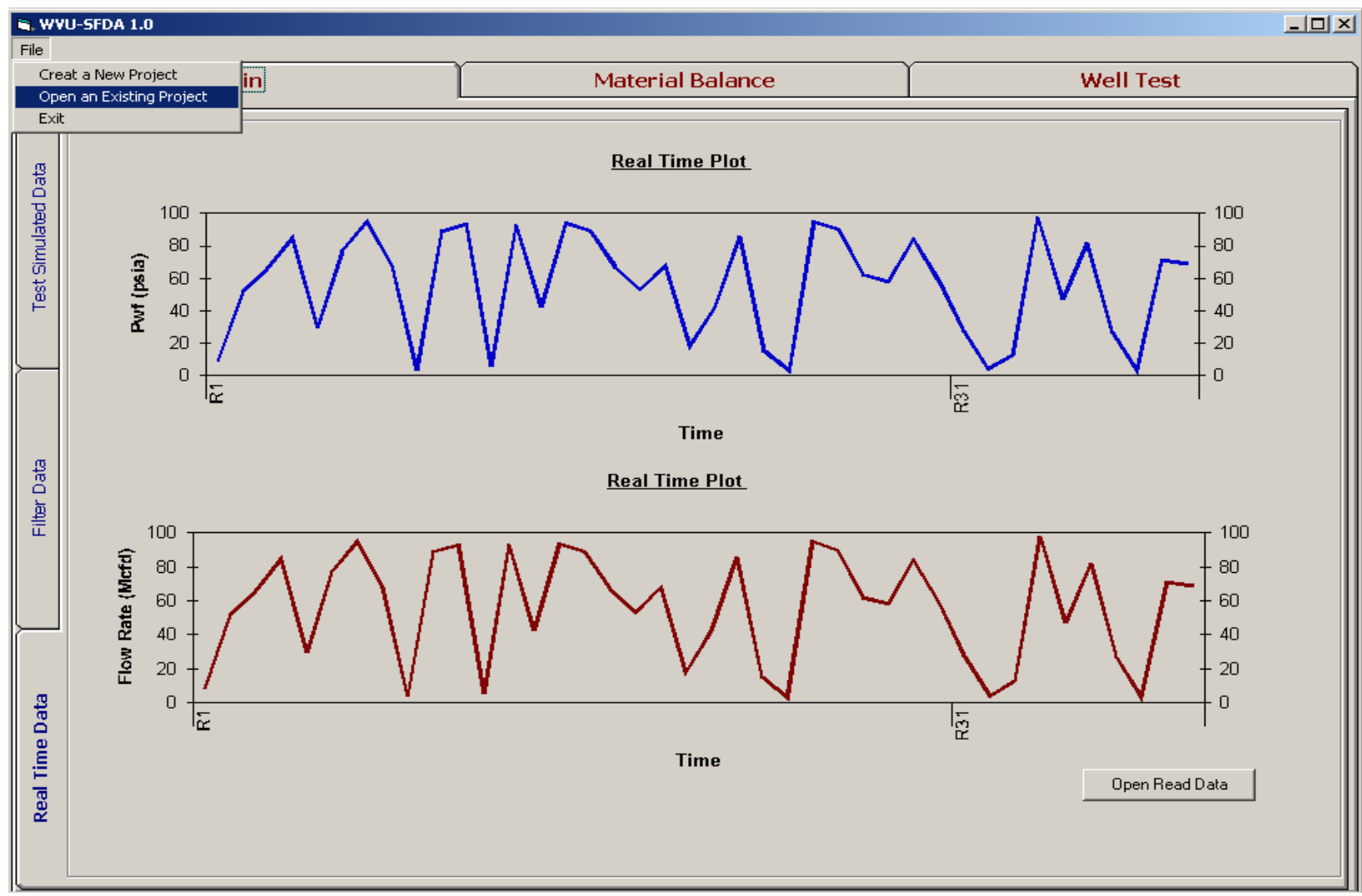

\section{E- MUL-SFDA 1.D}

\section{File}

Creat a New Project

Ореп ап Ехіsting Projert

\section{Exit}


Now you can select a file pertinent to real time data, double click on the file.
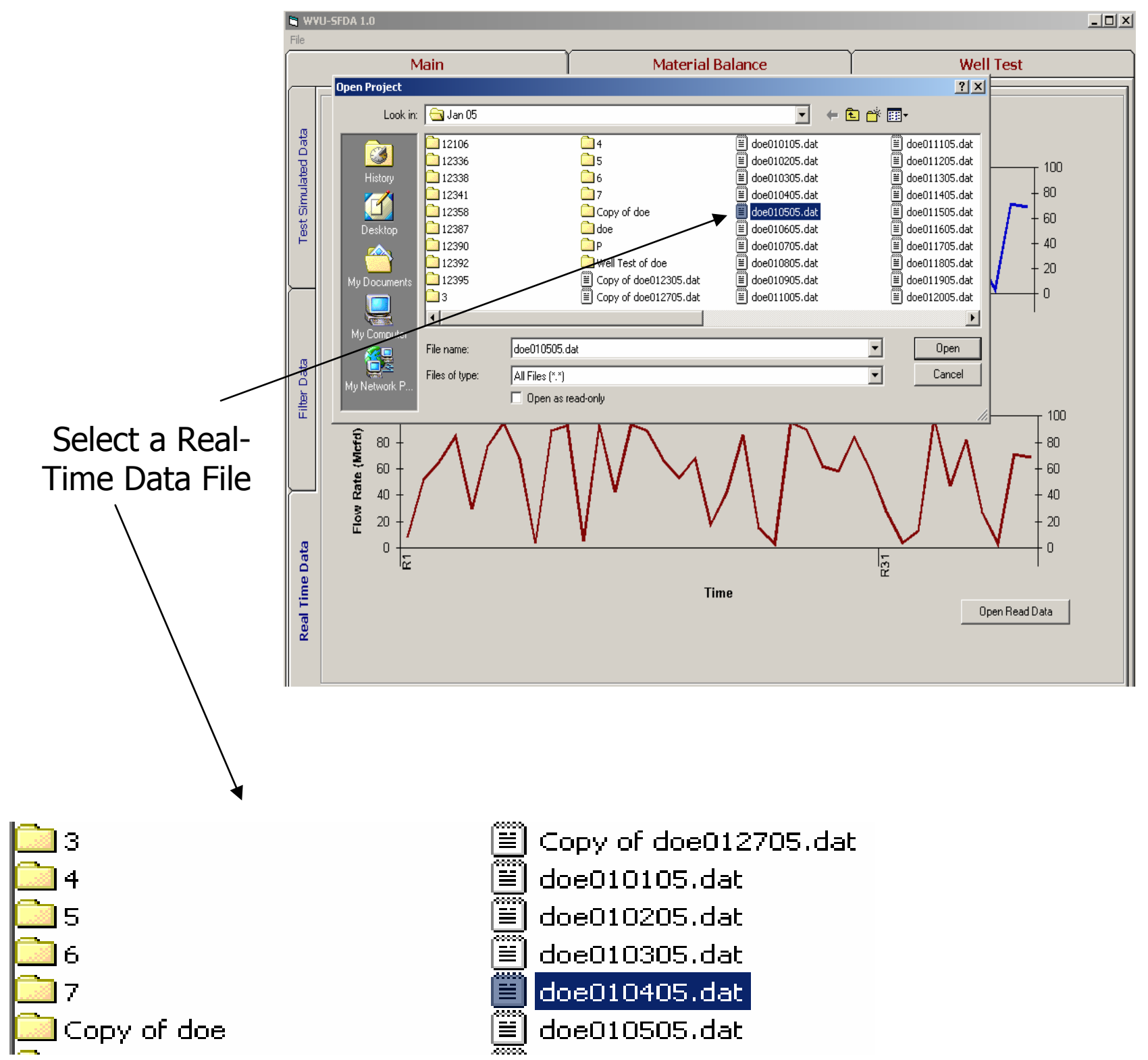

\begin{tabular}{|c|c|}
\hline 吾 & Copy of doe012705. dat \\
\hline 㕍 & doe010105.dat \\
\hline 奉 & doe010205.dat \\
\hline 䒠 & doe010305.dat \\
\hline 譬 & doe010405.dat \\
\hline 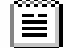 & doe010505.dat \\
\hline
\end{tabular}


Now, you can click on the "Open Read Data" button in order to see the changes in pressure and flow rate as the program reads data in real-time.

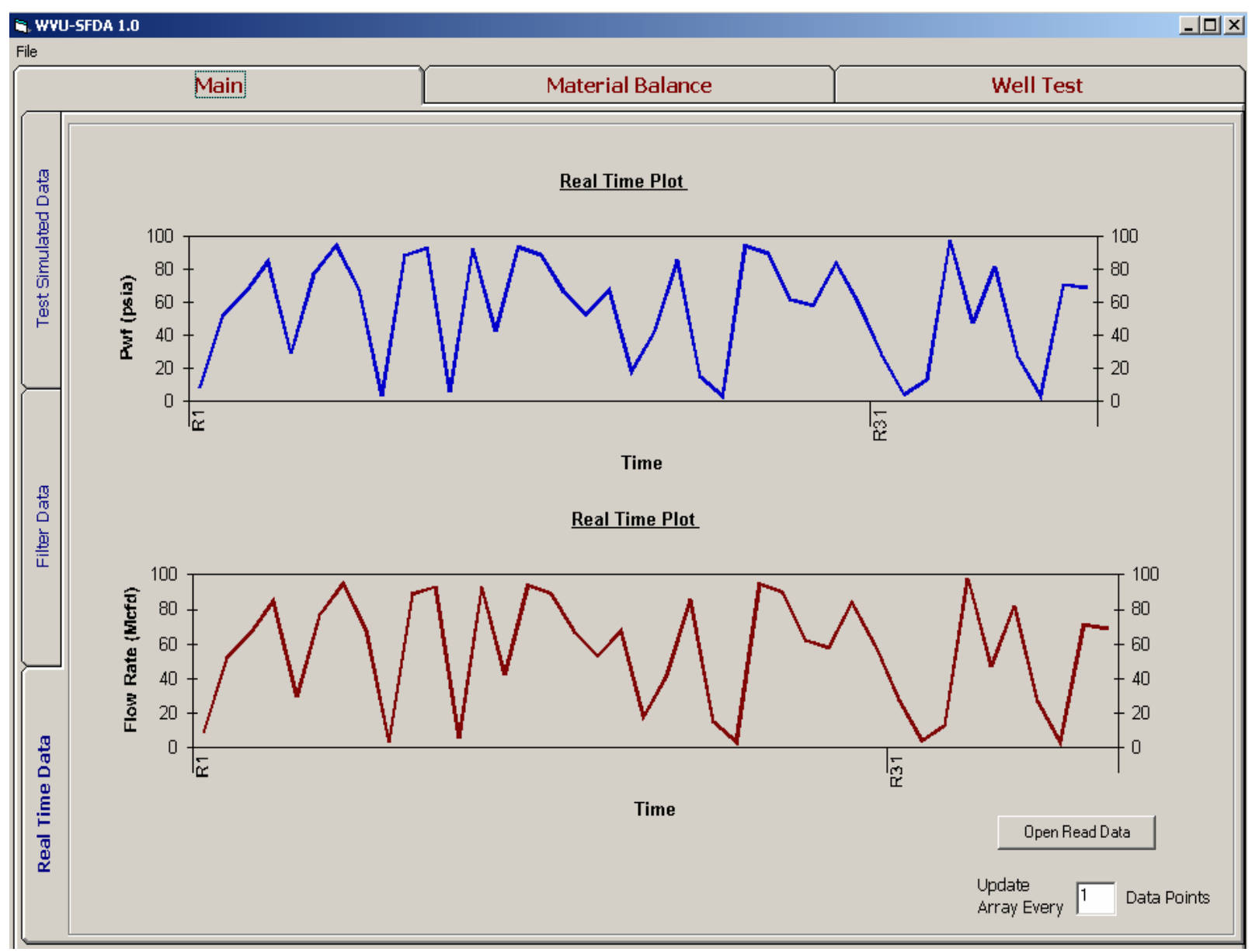


Now the program will be reading data and showing in a loop process in which every value will be read before plotting it.

Data in Real-Time will be shown.

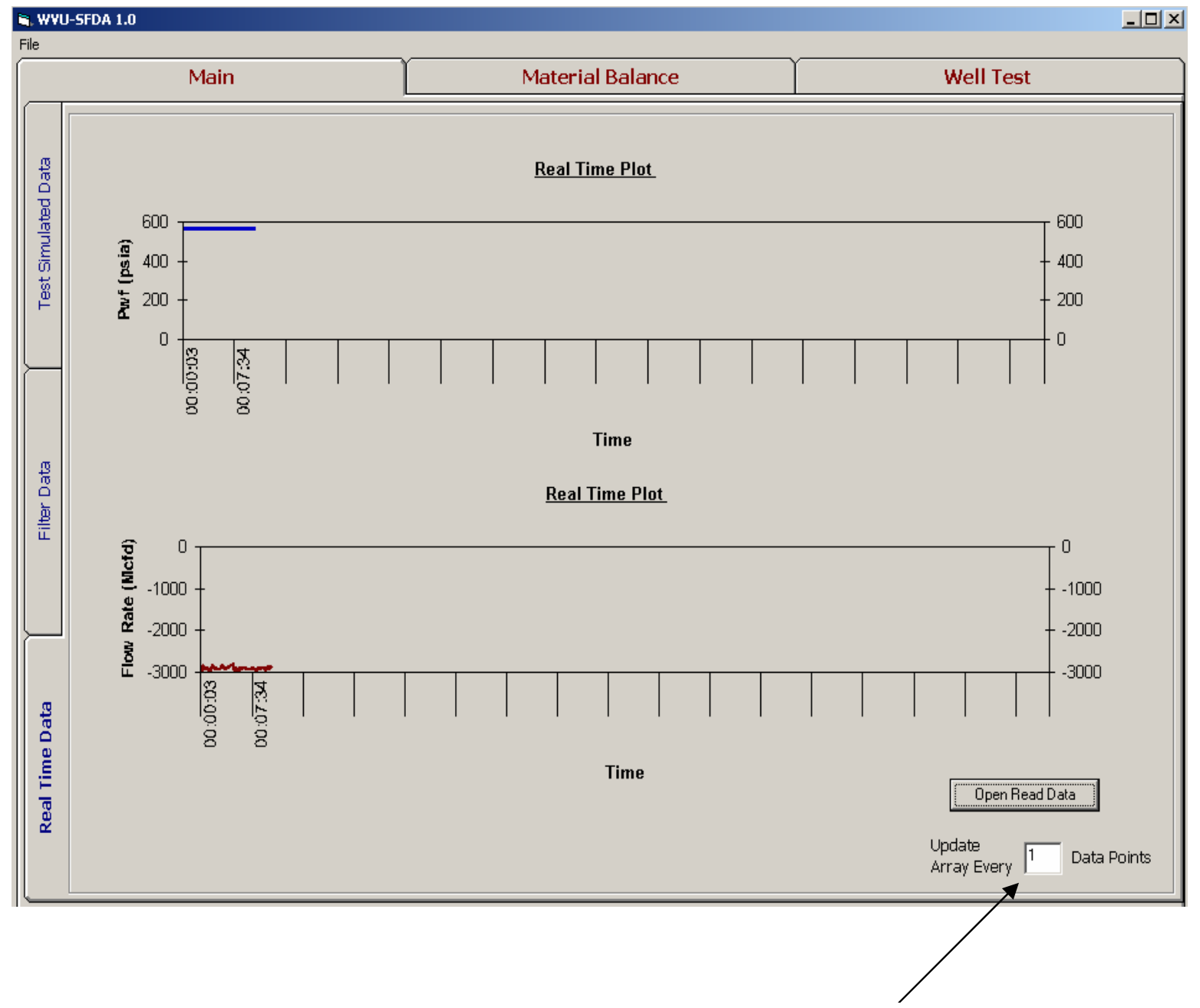

Right now you will see the program reading value by value. If you want to read every two data points or ten data points you can write the number of data points the program will read before showing the plot on the text box shown here and the program will read and show pressure and flow rate every data points you selected in order to make the program to run in a faster manner 


\section{Sub Tab: Filter Data}

SFDA will filter the data in real time and create a set of summarized data that will contain lesser data points and its plot will be very similar to the actual one. The process of filtering the data consists of the use of Preset values for $\Delta \mathrm{P}$ and $\Delta \mathrm{Q}$ to summarize the high frequency data while keeping its integrity. Preset values for $\Delta \mathrm{P}$ and $\Delta \mathrm{Q}$ represent the minimum difference at which pressure and flow rate should be recorded. Pressure and flow rate are recorded when the changes in such parameters ( $\Delta \mathrm{P}$ and $\Delta \mathrm{Q})$ are higher than the maximum presets $\Delta \mathrm{P}_{\text {Preset }}$ and $\Delta \mathrm{Q}$ Preset. Every value for pressure and flow rate is compared to a $\Delta \mathrm{P}_{\text {Preset }}$ and $\Delta Q_{\text {preset }}$ respectively to determine whether a value should be recorded or discarded. The program will automatically calculate changes in pressure and flow rate $(\Delta \mathrm{P}$ and $\Delta \mathrm{Q}$ ) continuously and decides whether such changes will be important to record new values that will be sent to a set of summarized data. As long as the changes are low, the program will not record any new value. Instead; it will assume a straight line between the old values and the new ones. New values will be sent to the set of summarized data when the program decides whether the changes in pressure and flow rate are high enough to record new values or low enough to ignore them. You can detect whether the program produced a set of summarized data similar to the actual one or not. If you do not agree with how the program summarizes the data, you can manually change the delta pressure and delta flow rate as many time as needed and redraw the summarized and actual one until such plots match.

This "Filter Data" tab will allow you to upload the summarized data from the day before and visualize the summarized data and actual one on the same plot. The picture box at the top represents Pressure vs. time. The one at the bottom represents Flow Rate vs. time.

After a day has passed and the program has created a set of summarized data, you can click on the button "Draw" in order to see how the summarized data differs from the actual one. 


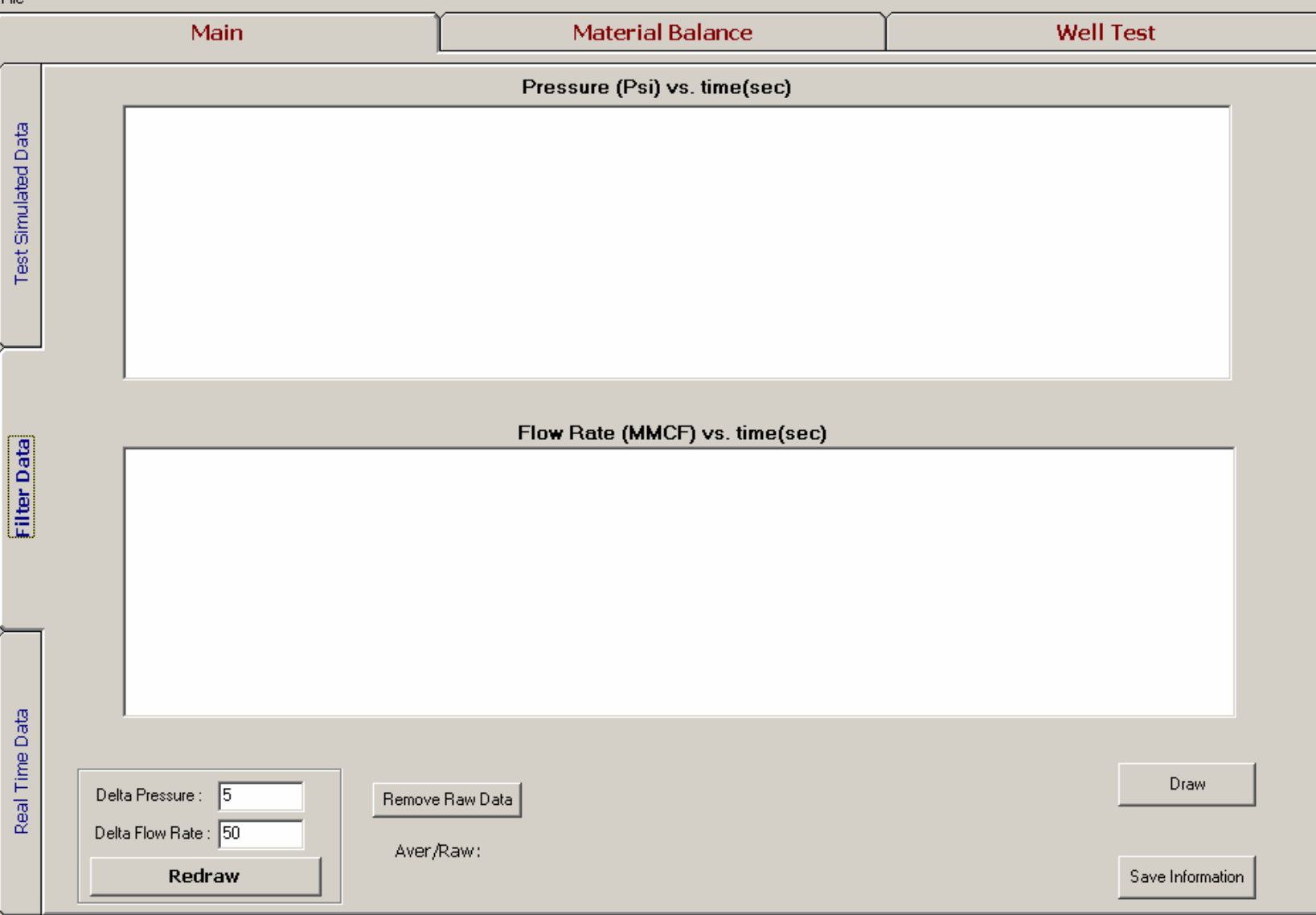

Once you click on the "Draw" button, plots of pressure and flow rate containing summarized and actual data will show up. The name of the "Draw" button will change to "Reset"

If you consider that the summarized and actual data do not match, you can change the delta preset values by changing the values of Delta Pressure and Delta Flow Rate and clicking the "Redraw" button. 


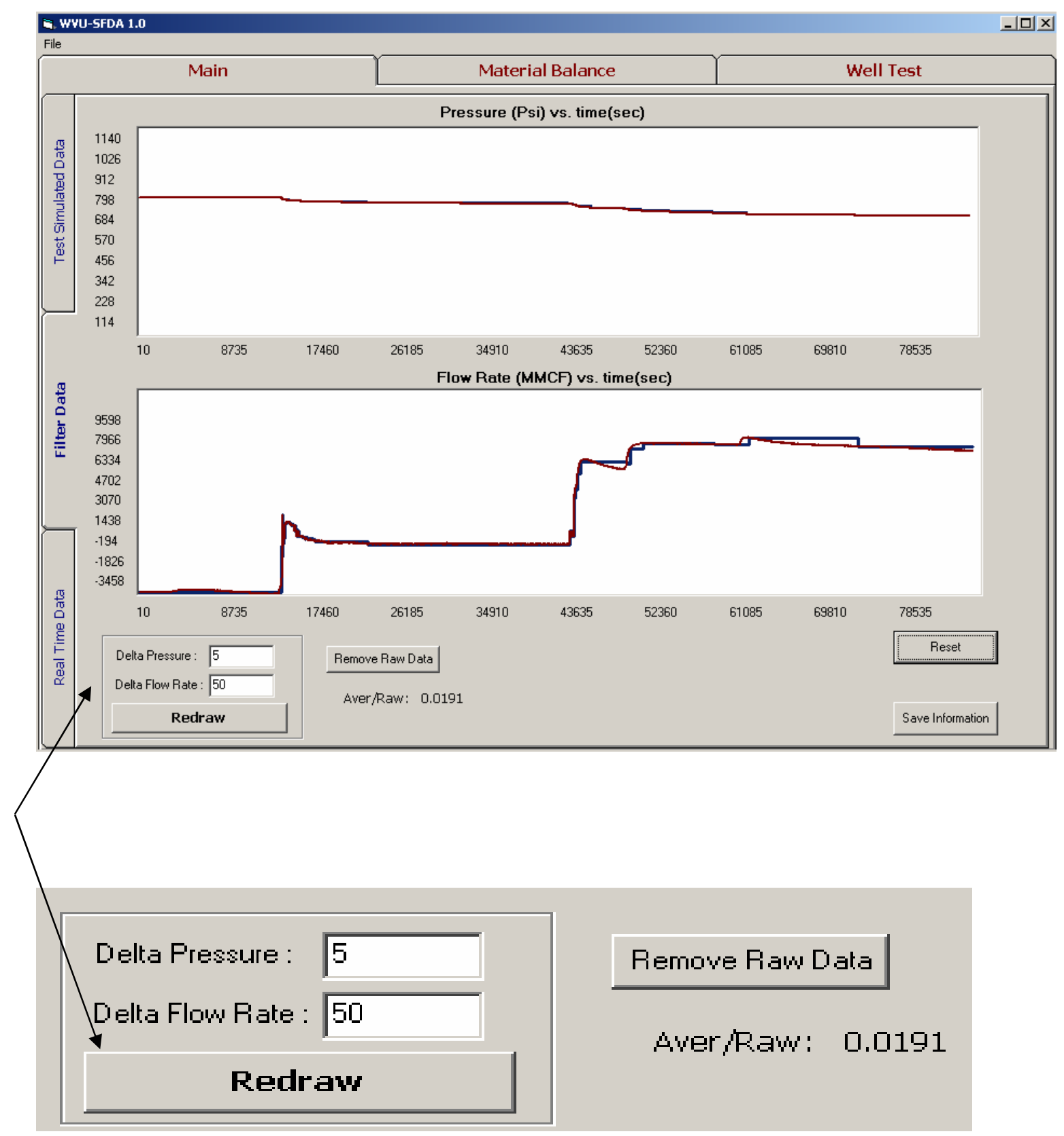

For this example, you will see the following result: 


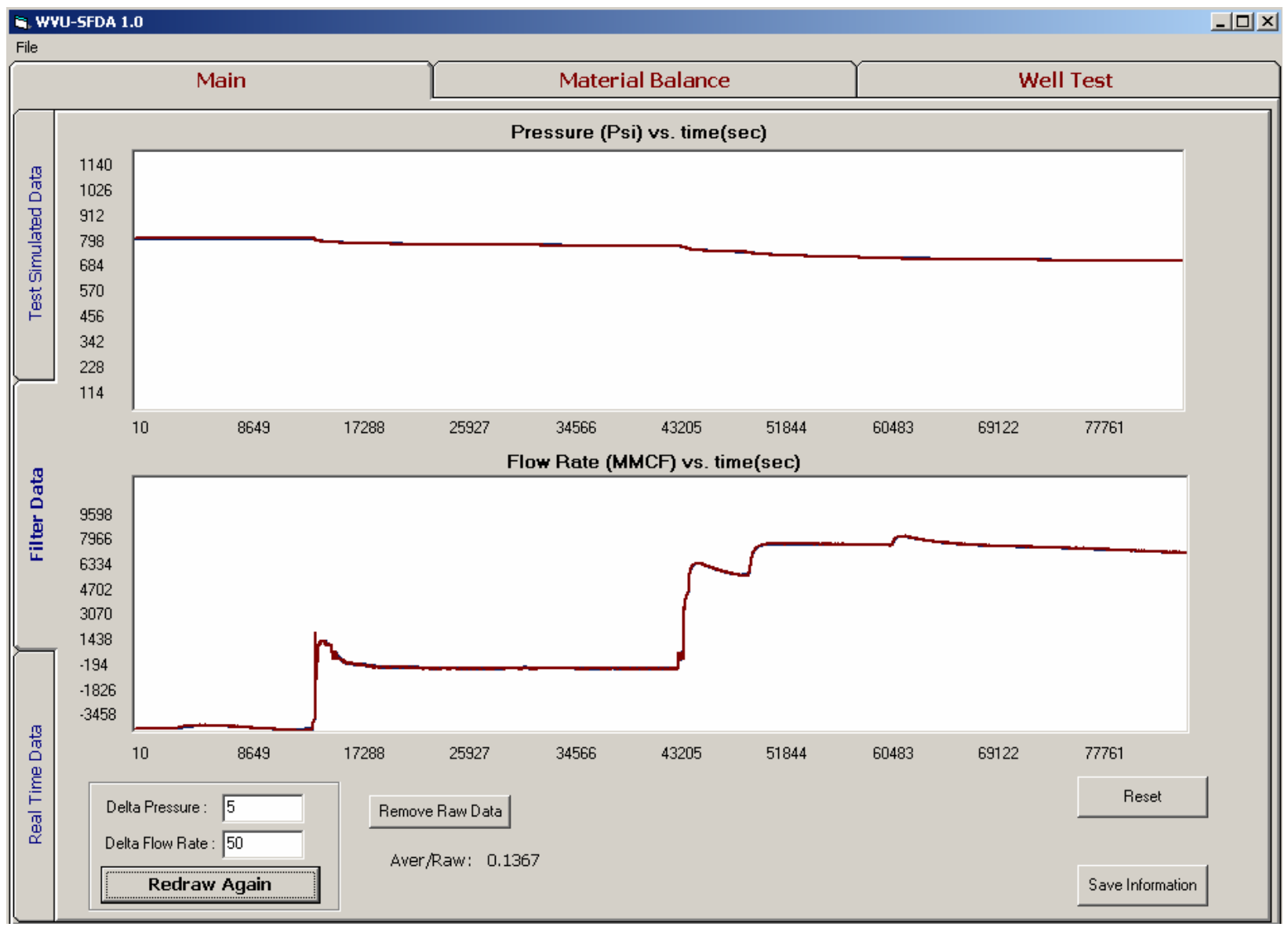

In addition you can click the button "Remove Raw Data", in order to see only the summarized data 
You will see now only the summarized data:

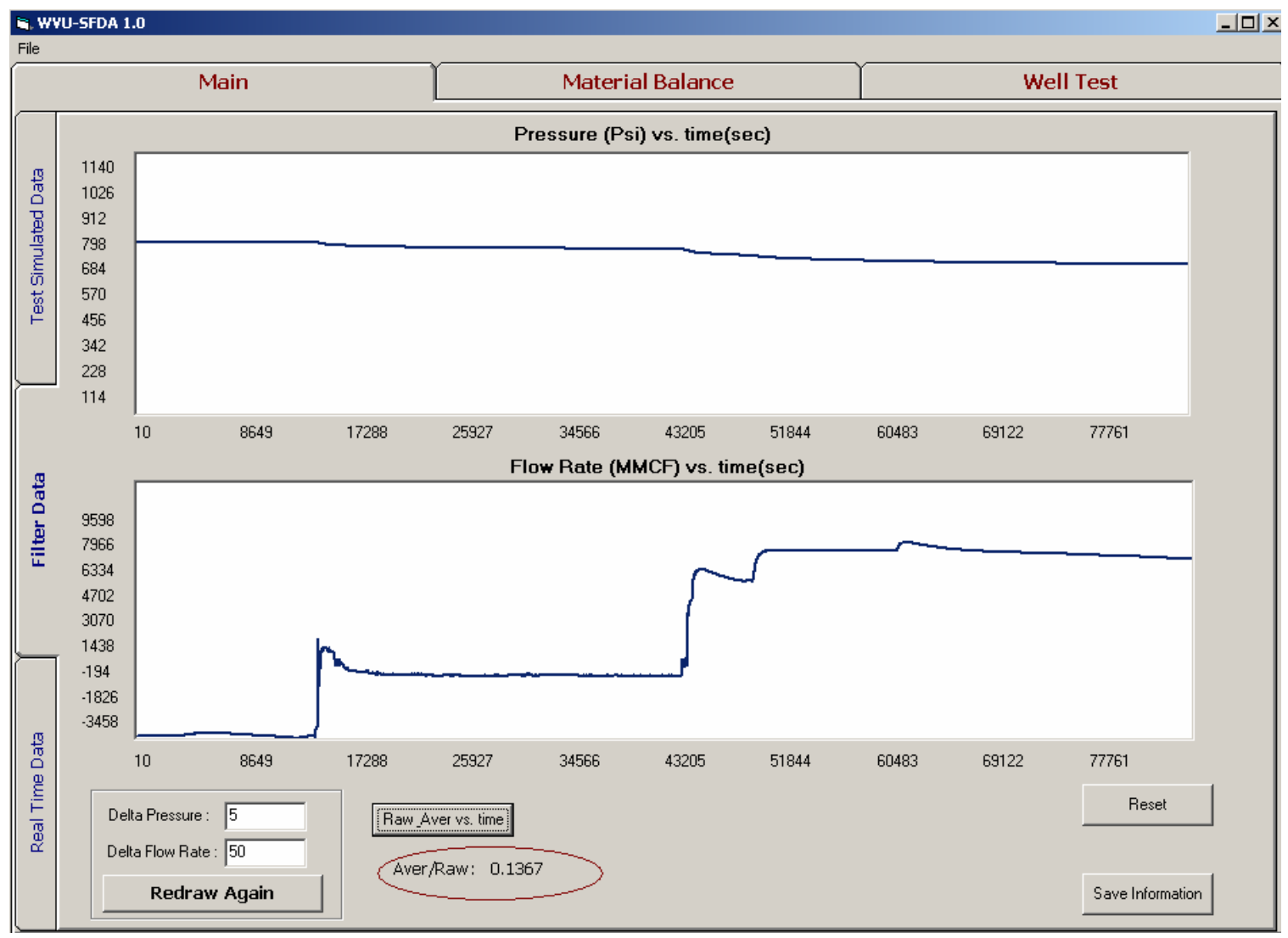

If you want to change the preset value again, you can do so and click on the "Redraw Again" button in order to see the new results:

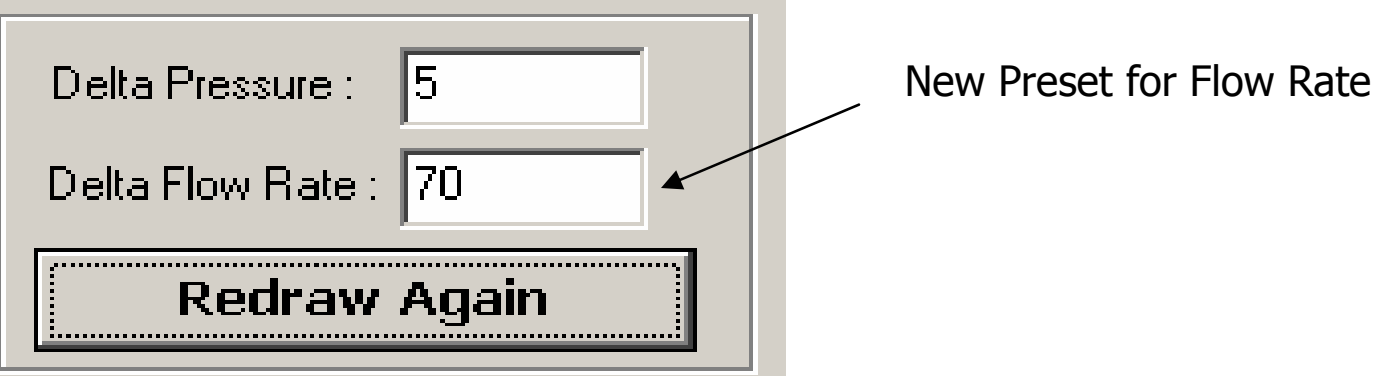


You can see the percentage of summarized data from the Aver/Raw captions surrounded by a red circle just below the "Raw Aver vs. time" button (the summarized data for this case contain $13.67 \%$ of the actual data)

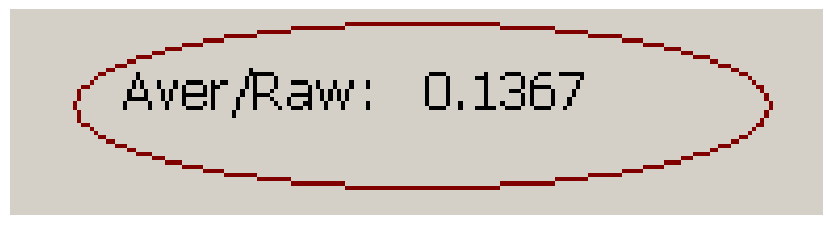

The new results are the following:

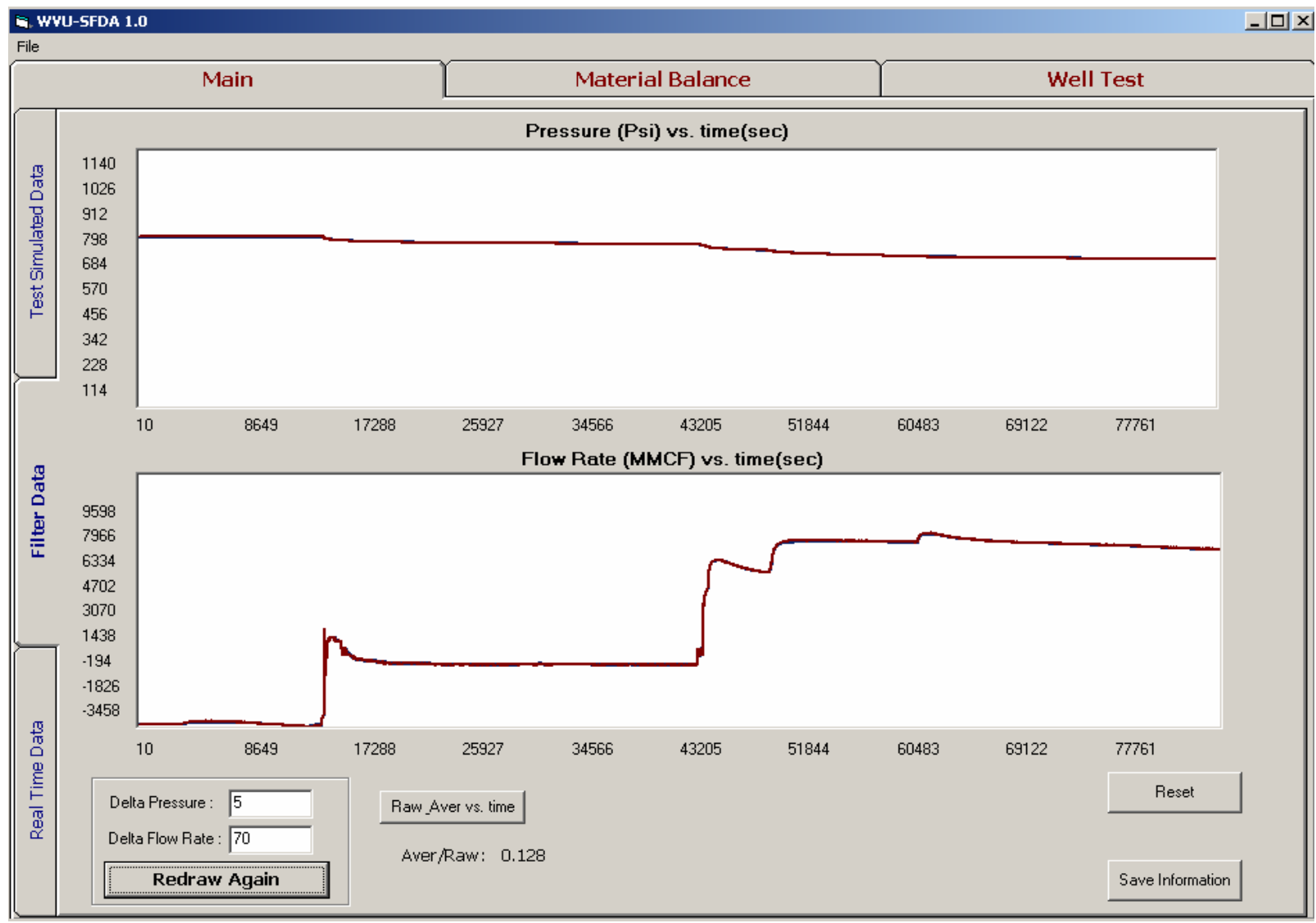

You can click on the same button but this time named: "Raw Aver vs. time" in order to see the results of summarized and actual data again. 
If you want to see again the result of the summarized data and actual one first given by SFDA, click on the "Reset" button.

\section{Fieset}

After clicking the "Reset" button, you will see the results SFDA first gave you:

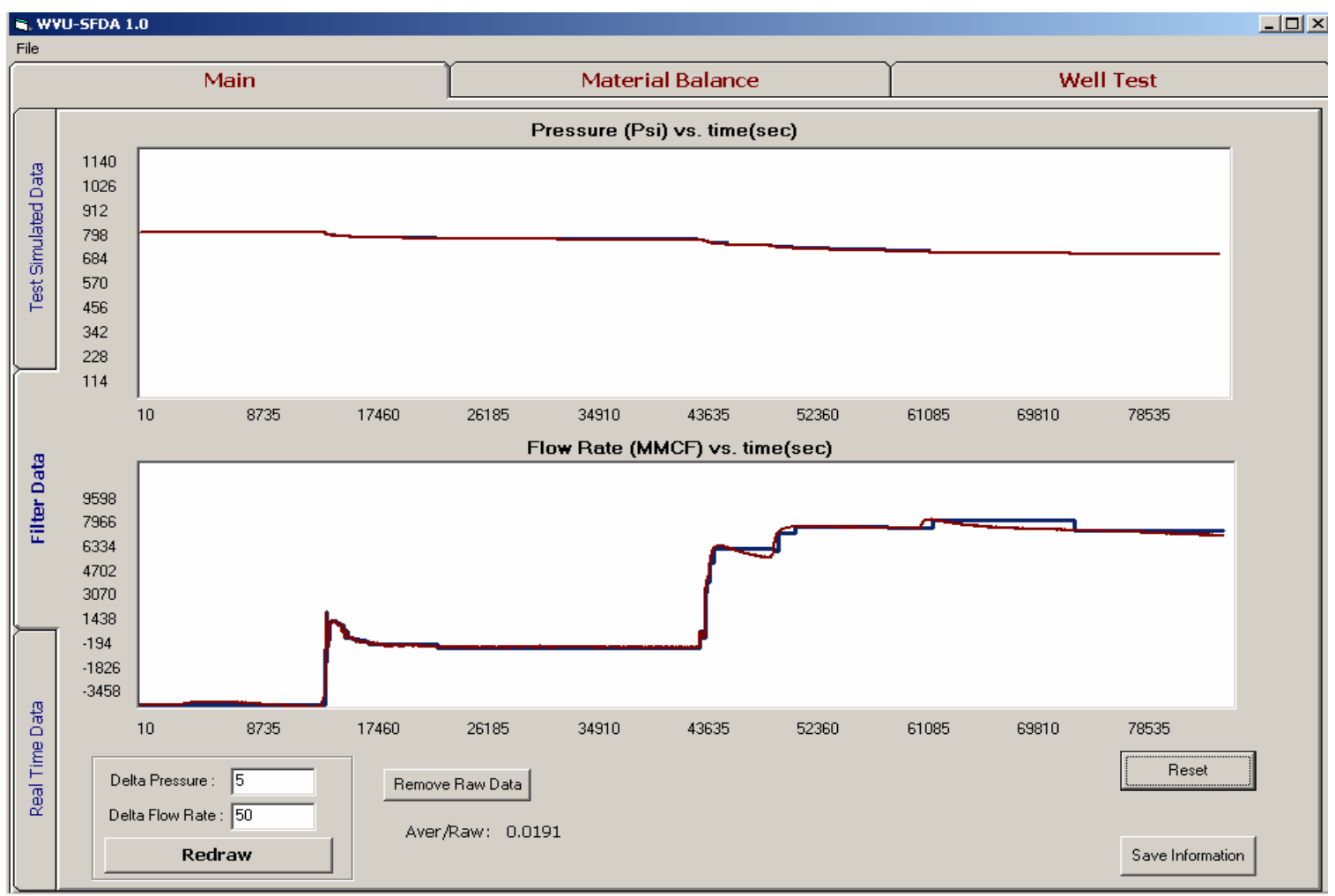

If you agree with the new results of summarized and actual data you can click on the button "Save Information" located just below the "Reset" button and the information will be saved. 


\section{Sub Tab: Test Simulated Data}

You can perform some test using data control environment from ECLIPSE in order to see how the summarized data varies from the actual one.

You will generate Real-Time data using ECLIPSE and read it using SFDA by first selecting the "Test Simulated Data" tab and then clicking on the "Select File" button:

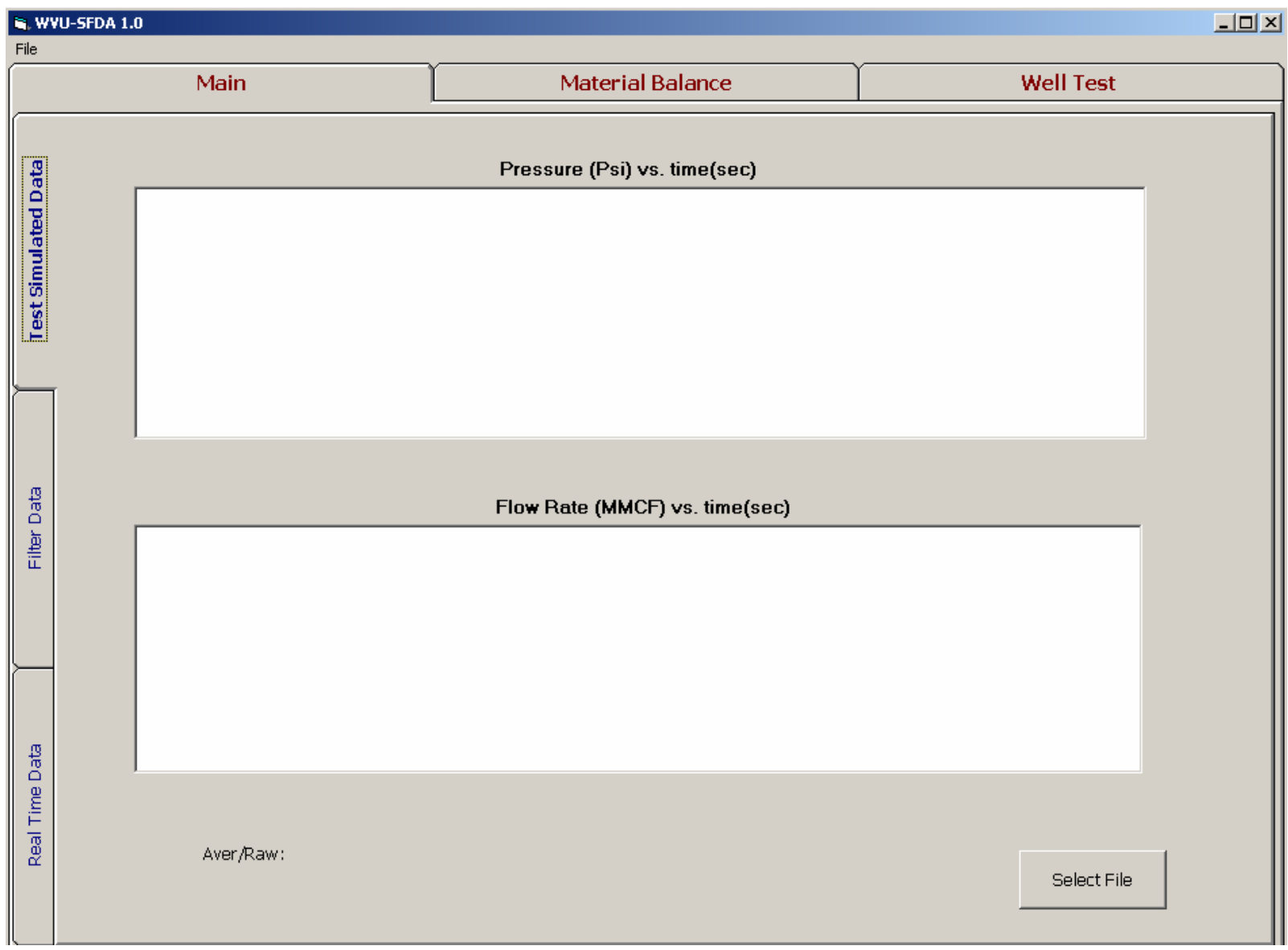


The time of information you should get from ECLIPSE is flow rate, pressure, and time in real time. You can get either daily, weekly, or monthly data.

Once you have clicked on the "Select File" button, you can find the file containing such information and upload it by double clicking on the file or click the command button "Open":

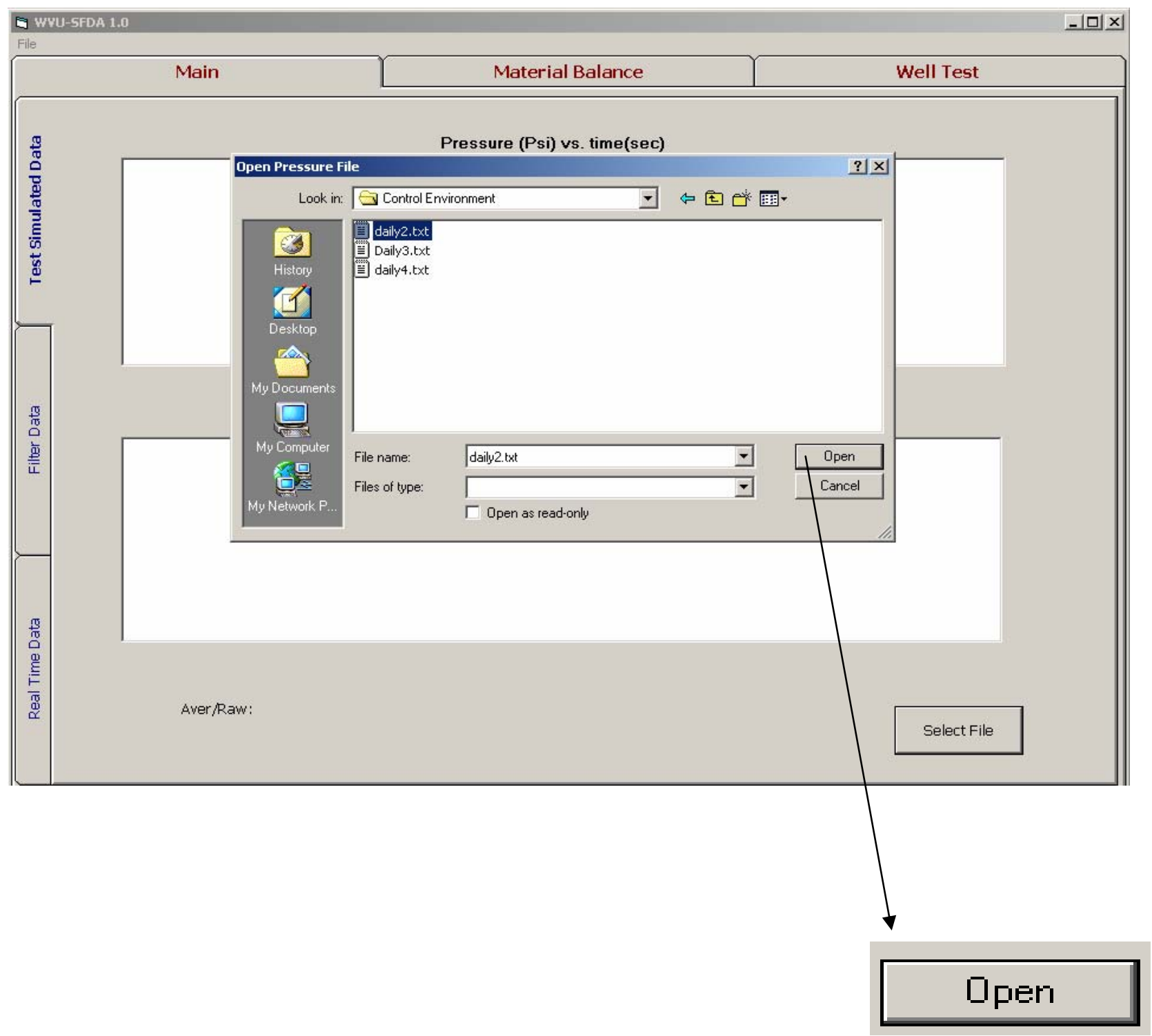


The following figure will show you the results of summarized data and actual one using real-time data generated by ECLIPSE. You can also look at the Aver/Raw caption to see the percentage of data points the summarized data contains. For the case below the summarized data contains about $0.61 \%$ of the actual one. The very small percentage is due to the very few changes in pressure.

For this case you will see the following results:

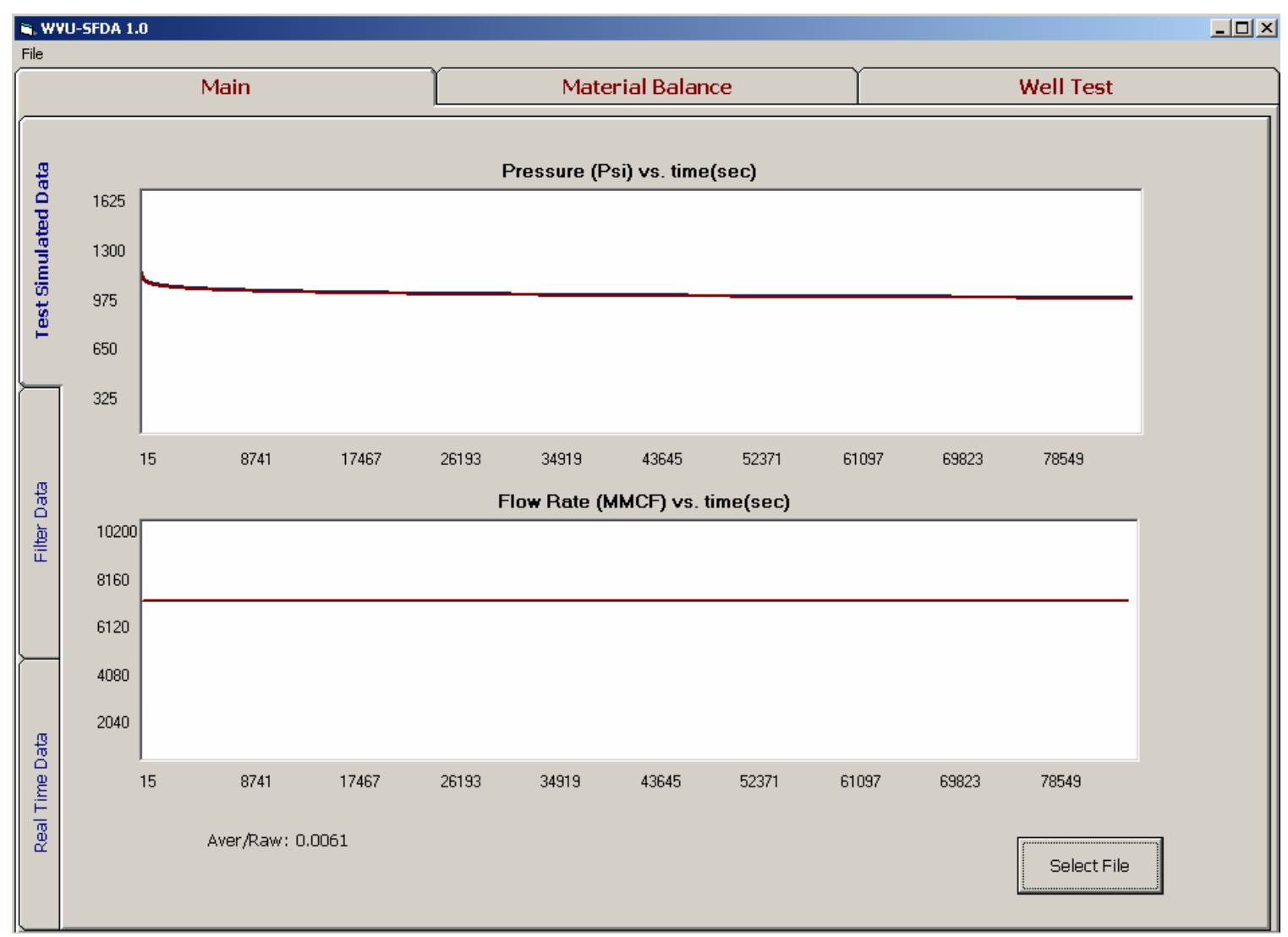




\section{MATERI AL BALANCE TAB}

This section will introduce you to material balance analysis and the estimation of gas in place versus time. Once the summarized data is obtained, the program has the option of showing pressure, flow rate, and gas in place versus time for every month. The user will have the option of detecting any abnormality occurred during a determined month from the pressure, flow rate, and gas in place profile. In addition, on the upper part of every graph, the differences of changes in their parameters will be shown.

Gas in place is a value that should remain constant at all times. Monitoring such a parameter will allow detecting whether the gas in place decreases or remains constant. You might interpret a decrease in gas in place as the existence of a leak. In such a case, the operators can be notified and the appropriate actions can be taken right away. Gas in place in Real-Time can be observed as data is entering the system. SFDA estimates the values of gas in place and shows it in a graph as the pressure and flow rate parameters are shown by the program.

On this section you can also upload the summarized data in order to perform decline curve analysis which can be used as a starting point to set the alarms systems. Real-Time data can be compared to the decline pattern in order to detect those situations when the real-time data does not lie on the decline trend. The material balance tab will consists on three sub tabs: "Gas in Place in Real Time" that will show the gas in place in real time, "Decline Curve Analysis" in which you will be able of uploading the summarized monthly data and selecting the appropriate parameters to draw the decline curve, and the "Production Profile" where you can upload the monthly summarized data to observe the profile for pressure, flow rate, and gas in place. 


\section{Sub Tab: Gas in Place in Real-Time}

You can now press on the "Material Balance" tab and on the "Gas in Place in Real-Time" sub-tab in order to see how SFDA estimates Gas in Place in RealTime:

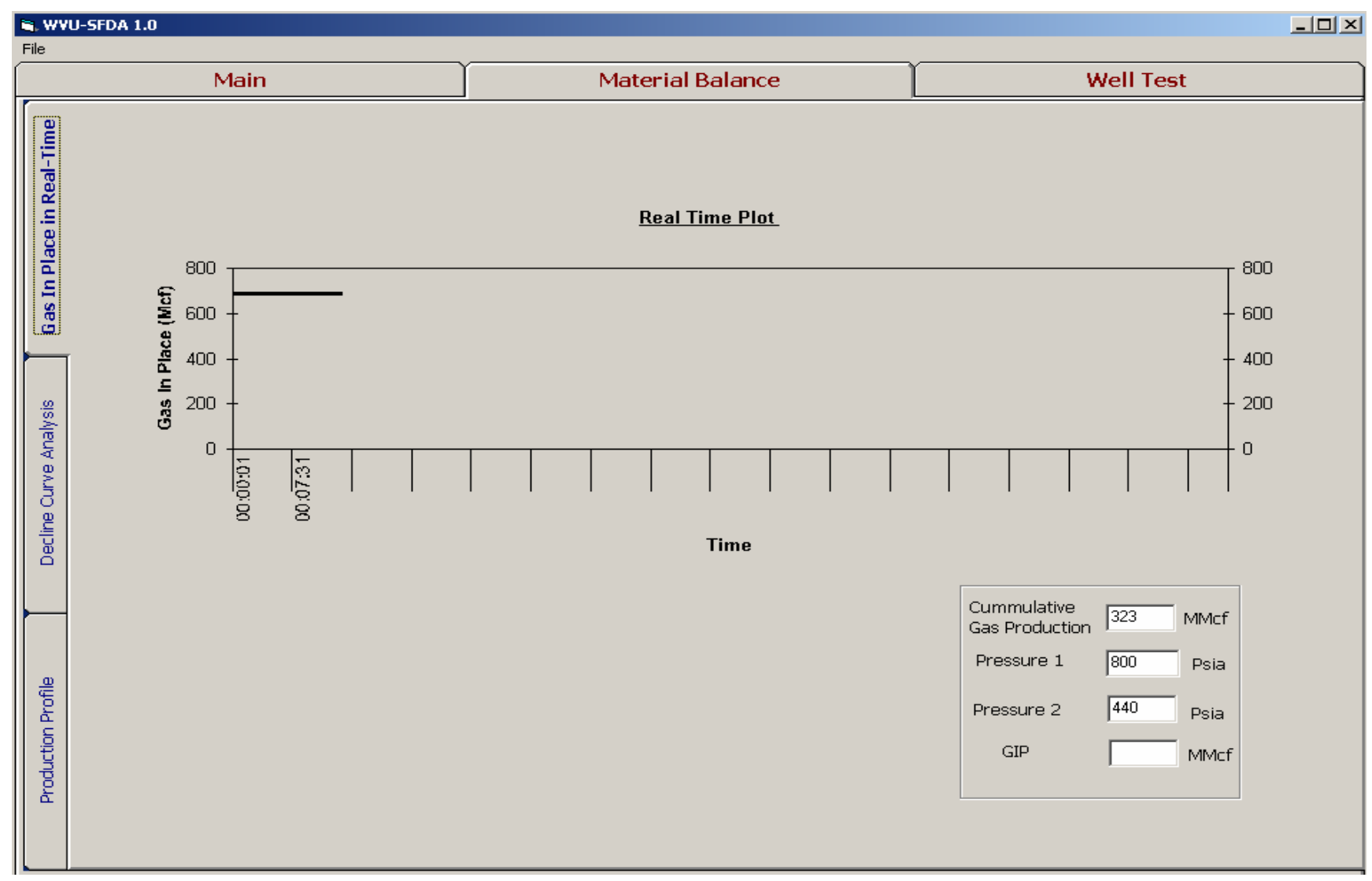

\section{Sub Tab: Production Profile}

If you run all the files containing Real-Time daily data for a month and then create files that contain summarized data for every month, you can show the pressure, flow rate, and gas in place profiles of the summarized data.

The first step will be to select the "Production Profile" tab and to click on "SELECT FILE" button in order to upload the summarized monthly data: 


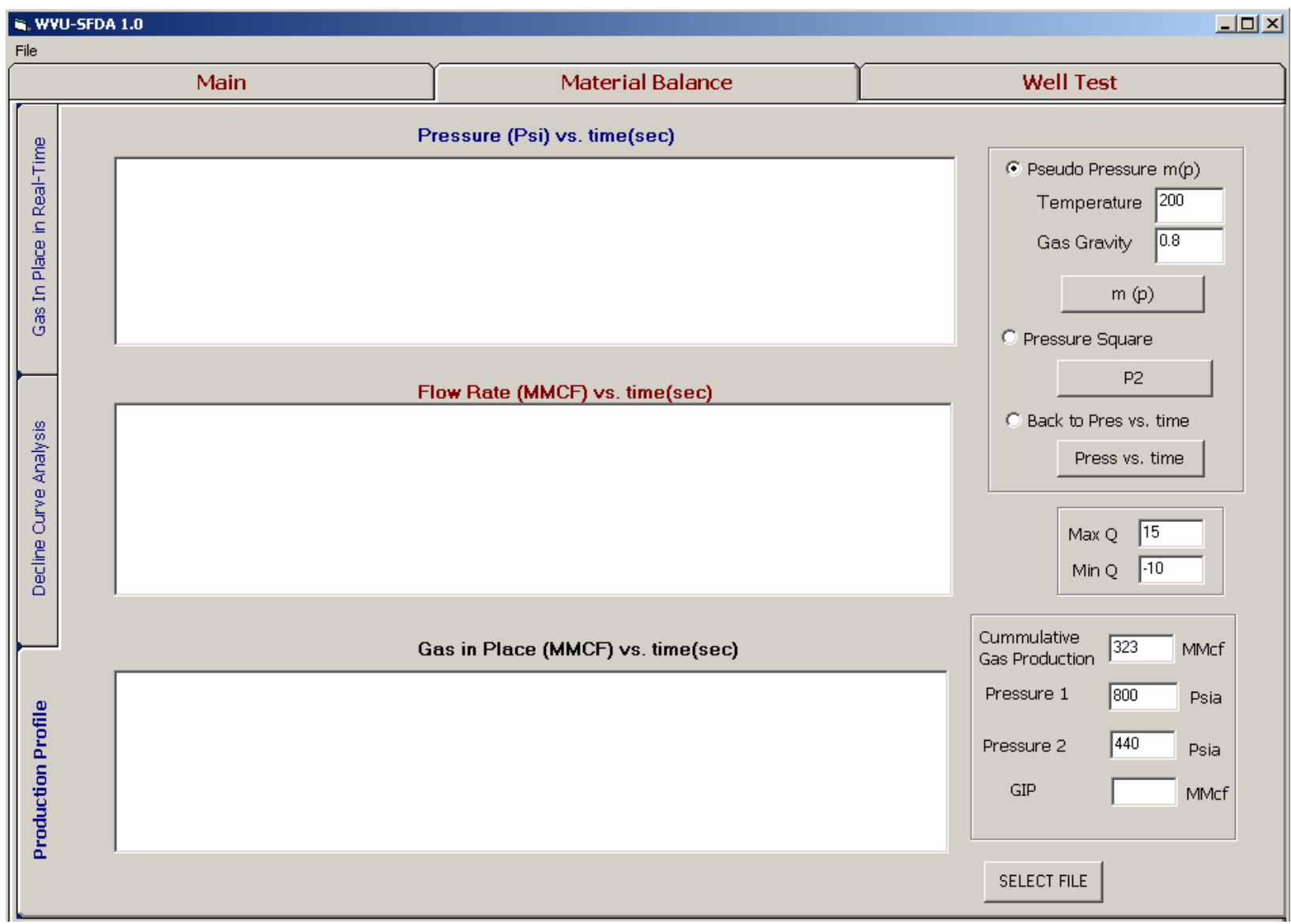

After clicking on the "SELECT FILE" button, you can find and upload the summarized monthly data: 


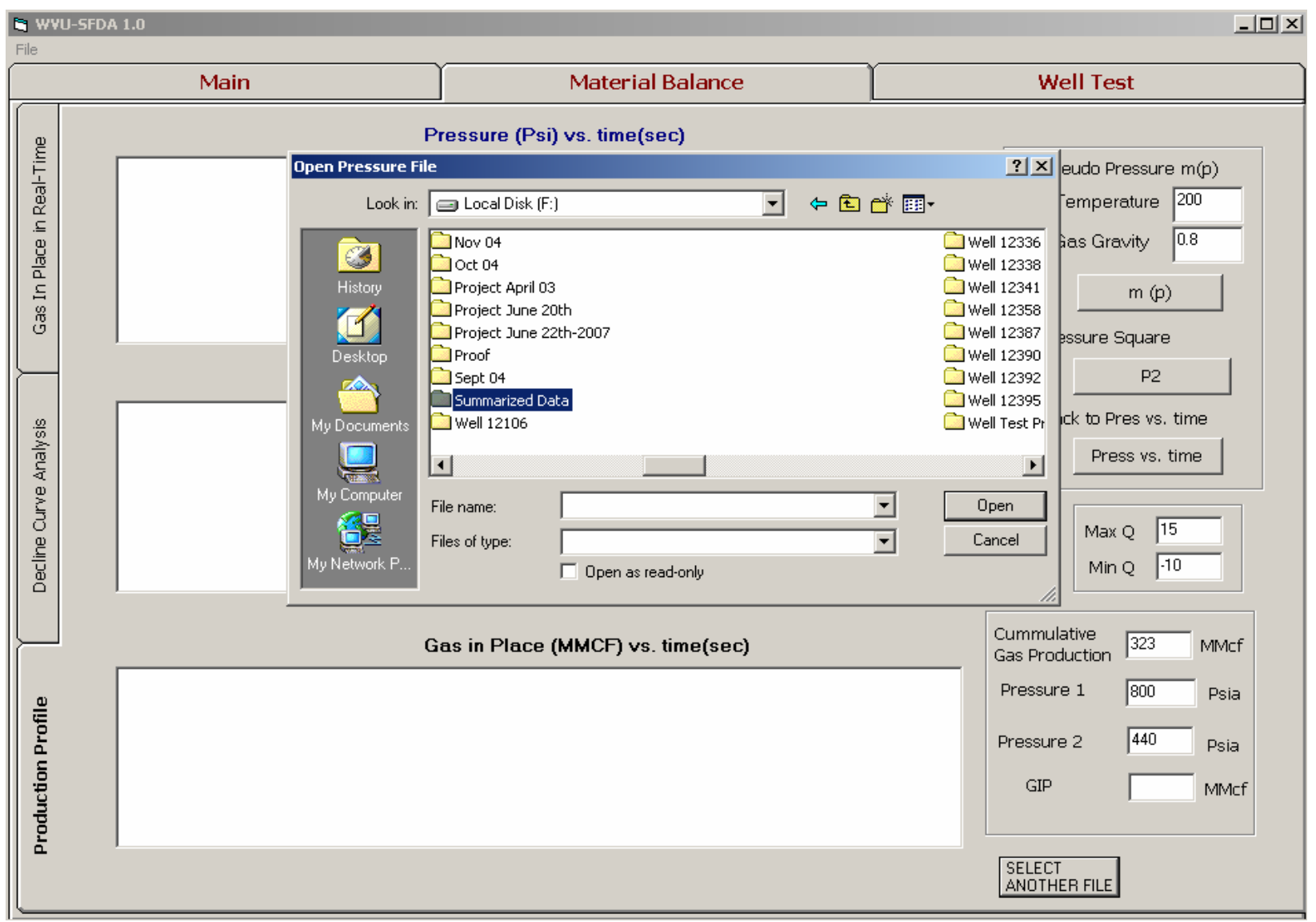

\section{Open Pressure File}

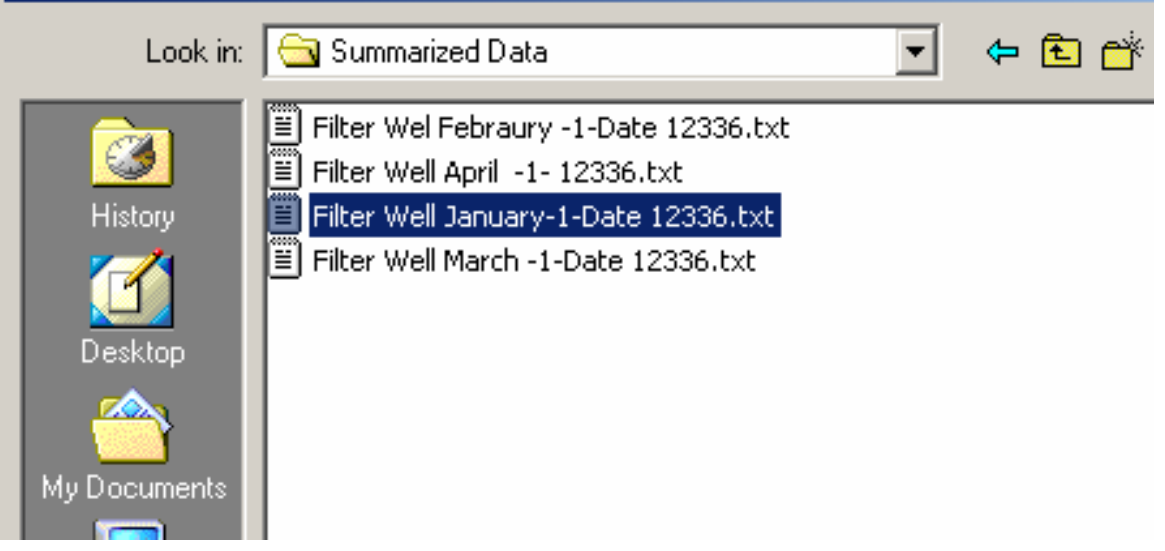


After double clicking on the file selected you can see the profile for pressure, flow rate, and gas in place:

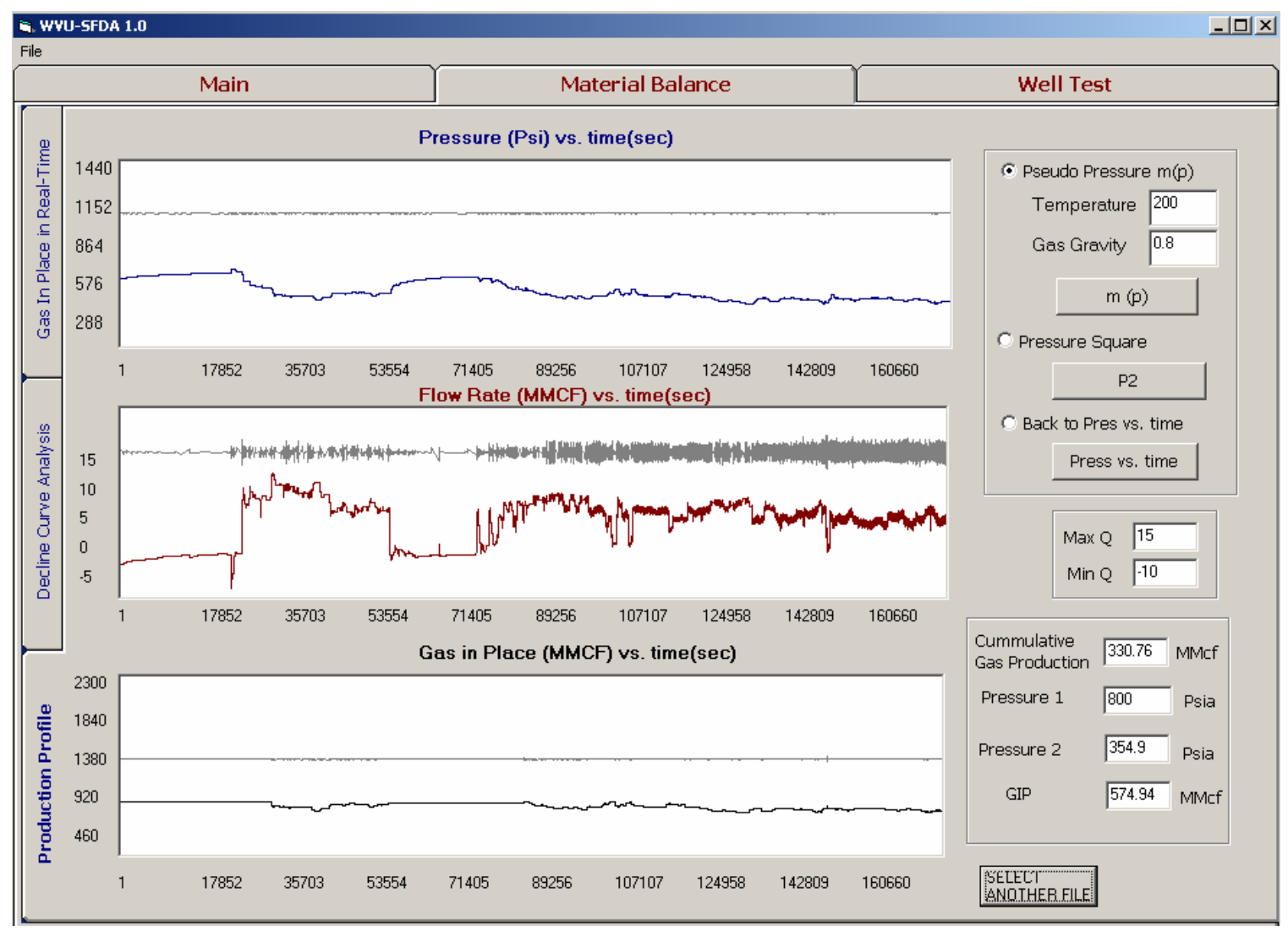

You can select the parameters of Pressure 1 and Pressure 2 that you will use to estimate gas in place. Pressure 1 will be a constant value and Pressure 2 will be the first value used to calculate gas in place, and when the software reads the next value of pressure; the gas in place will be calculated between this new value and Pressure 1 and so on. Also the value of cumulative production needs to be inserted in order for the program to make the calculations: 


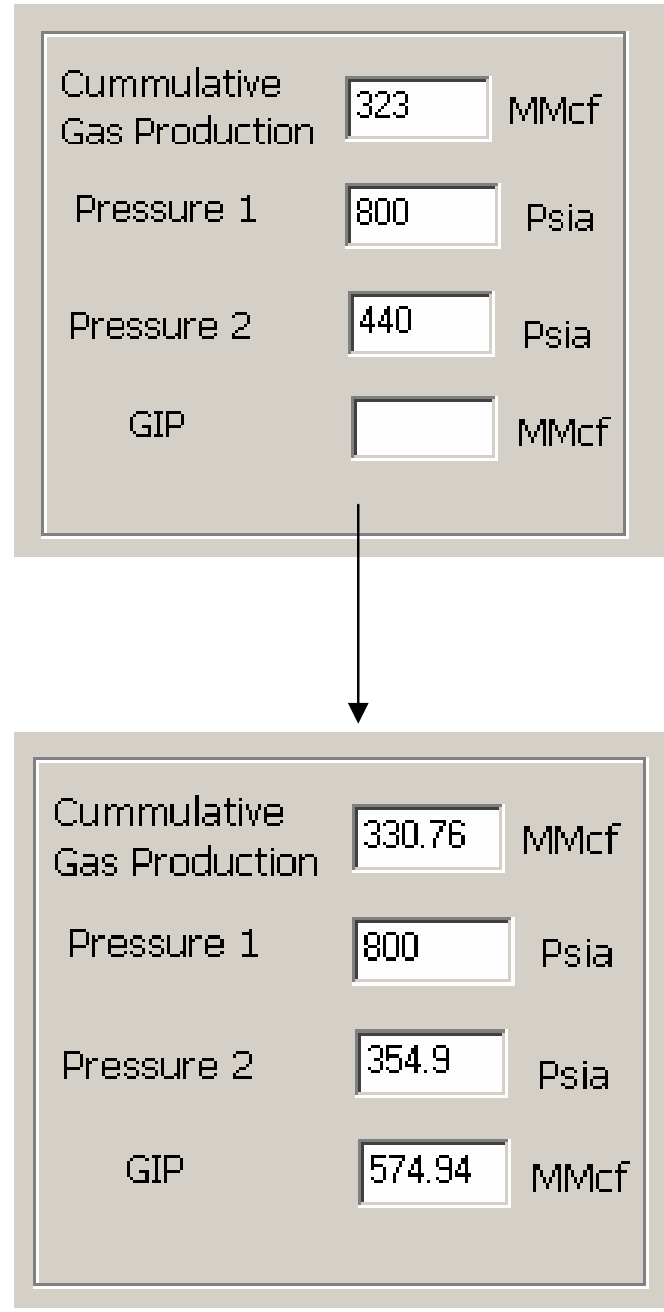

$$
\text { = Values before running SFDA }
$$

At the end, SFDA will gave you the values of cumulative production, Pressure 2, and gas in place

$=$ Values after running SFDA 


\section{Pseudo Pressure Profile Option:}

SFDA has the option of changing the plot of pressure versus time to pseudopressure versus time. In several cases during pressure transient analysis for gas production it is recommended to perform the well testing analysis using pseudo pressure values. The well test section of the software will allow the user to plot pseudo pressure versus pressure and draw the Horner plot from an area selected by the user.

You can change the pressure profile to pseudo pressure profile by selecting the

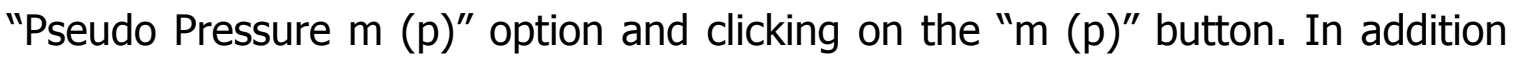
you will choose the values for temperature and specific gas gravity the program will need to calculate pseudo pressure values.

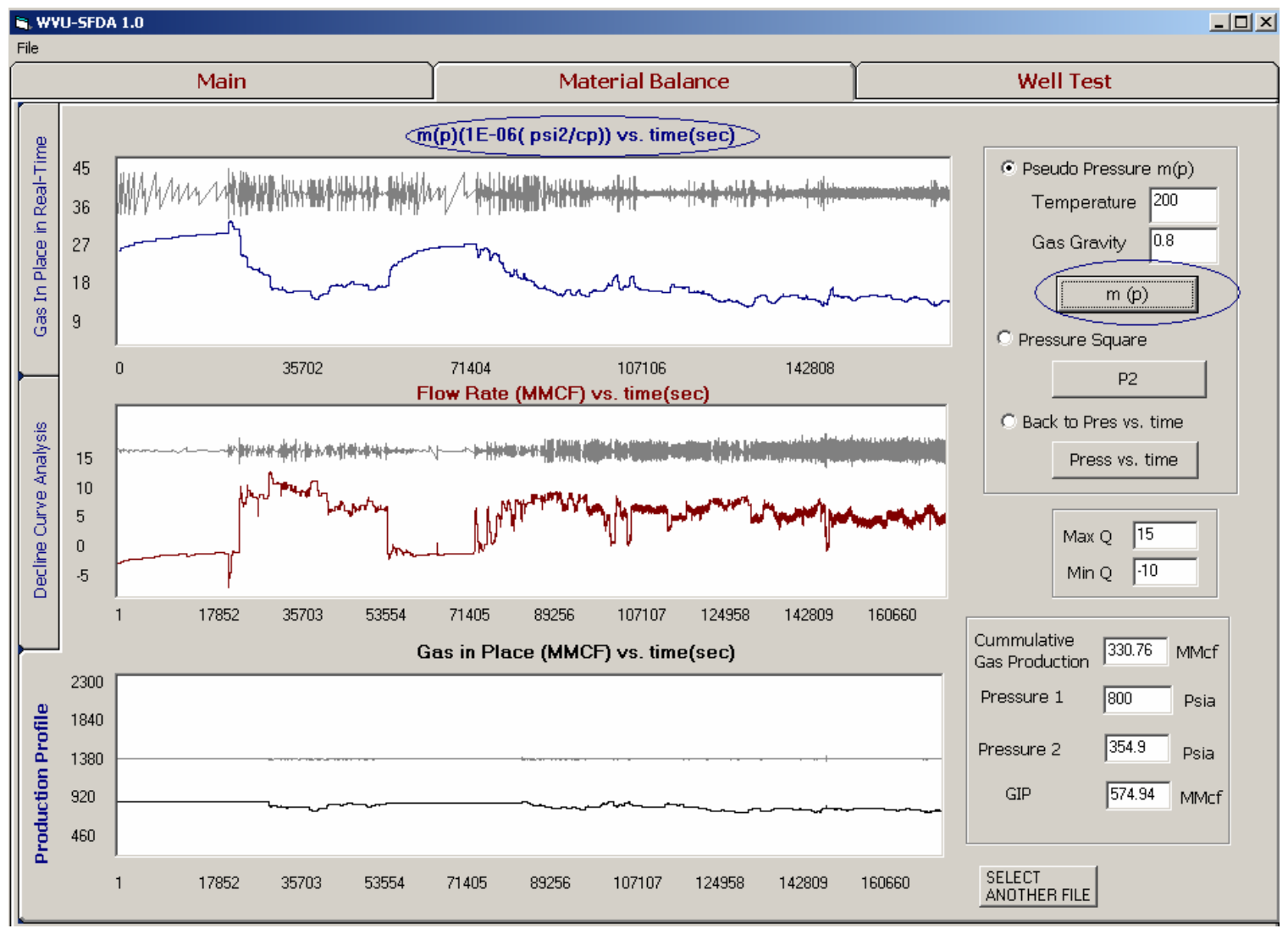




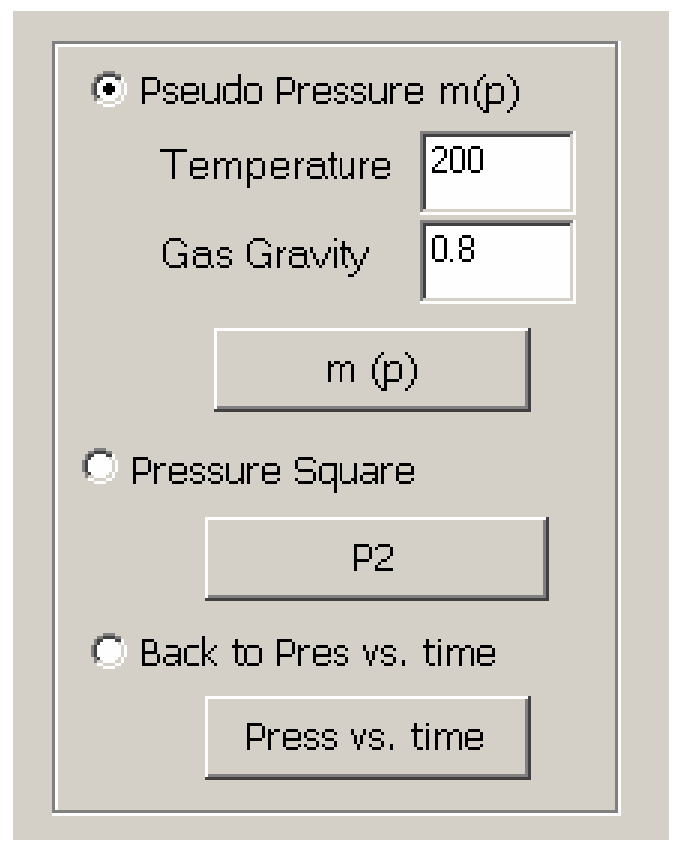

\section{Delta Pressure Square Profile Option:}

SFDA has also the option of changing the plot of pressure versus time to the square of pressure versus time. This is used in some well testing analysis to obtain permeability and skin factor from drawdown periods You might want to change the pressure profile to pressure square profile. Again, you select the option "Pressure Square" and click on the "P2" button.

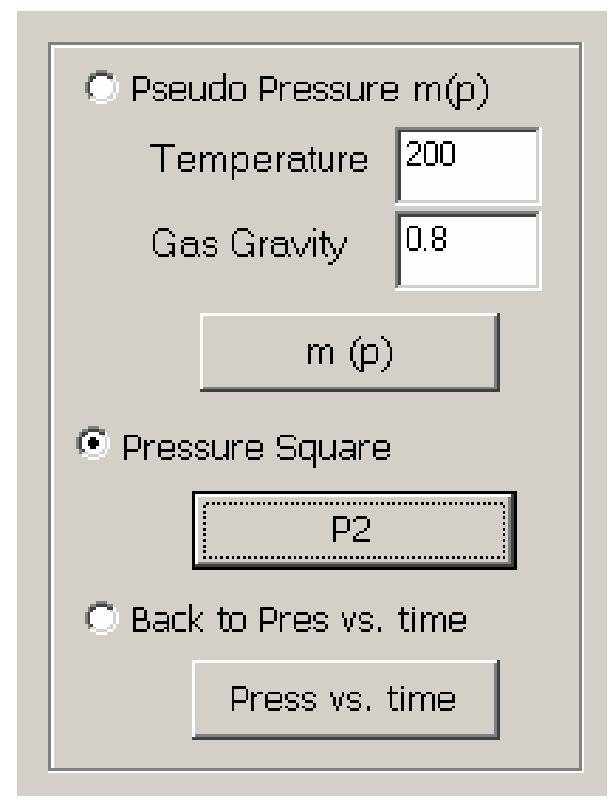


If you want to draw the profile for pressure back again, you can do so:
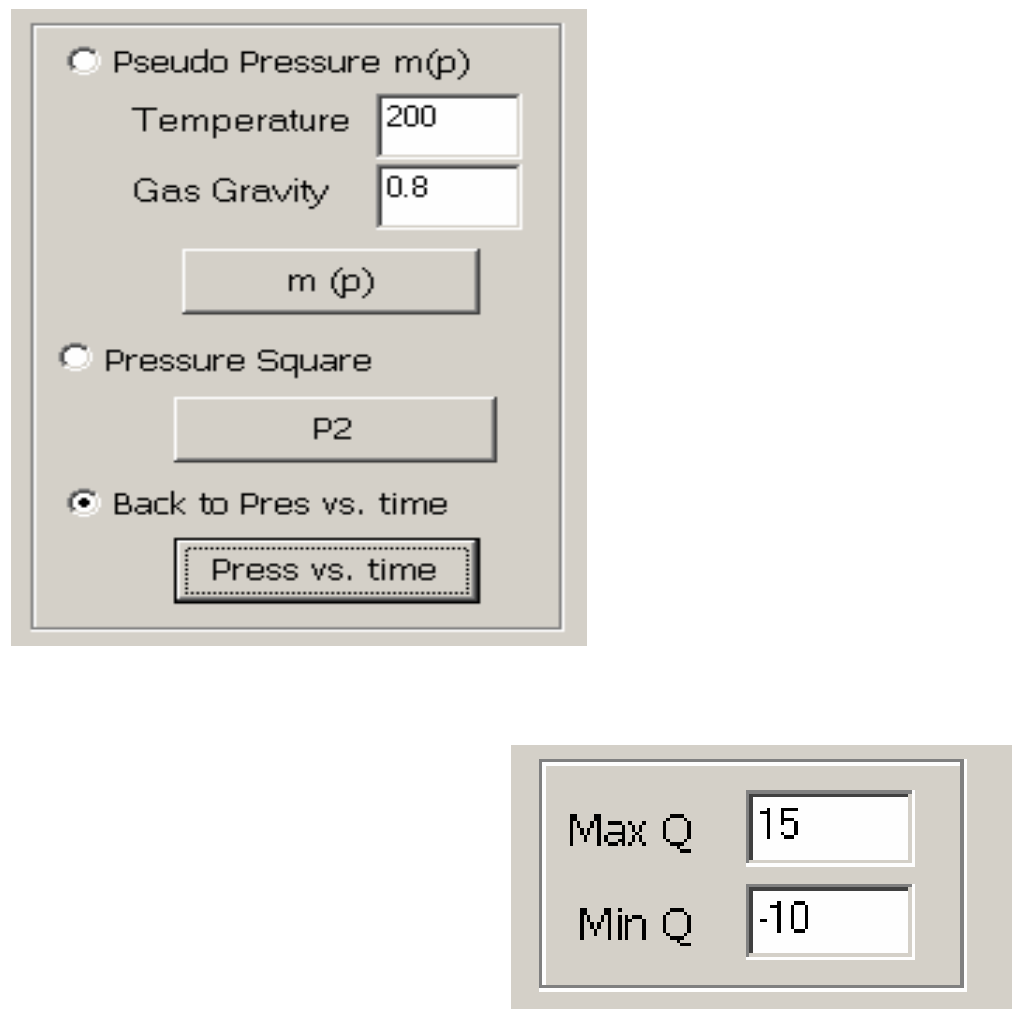

You can change the coordinates for flow rate since flow rate can read very high positive and negative values

You can also upload the summarized data and perform decline curve analysis by first selecting the tab "Decline Curve Analysis" and then select the "Q vs. time" option and click on "Select File" button 


\section{Sub Tab: Decline Curve Analysis}

SFDA will have the option of performing decline curve analysis to predict future gas production. The user will have the option of downloading the monthly production summarized data that the software can provide and choose the appropriate parameters such as b, Qi, and Di to draw the decline curve. Once the decline is drawn, future prediction about gas production for a year period can be made. The date at which the initial flow rate is selected will also be saved for future analysis.

Two types of decline analysis can be used by the user: Production Flow Rate versus time, and Production Flow Rate versus Cumulative Production. The first one can be used for new wells to calculate the expected production profile over a certain period of time. On the other hand, the flow rate-cumulative production plot can be used to calculate expected production profiles for production wells whose production history is available. 

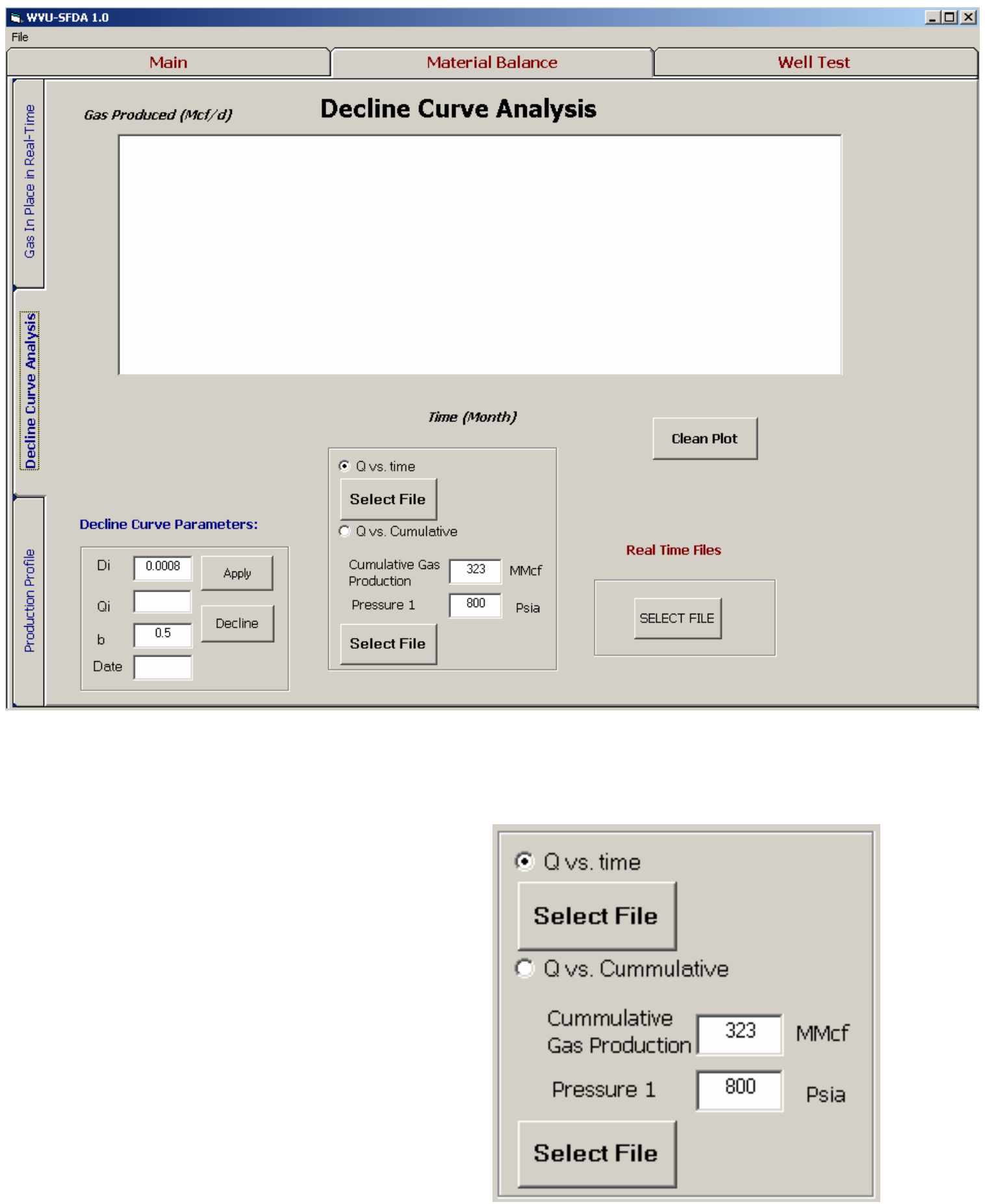
You can now look for the monthly summarized data and start selecting the files.

First double click on the first file; for this case it would be January:

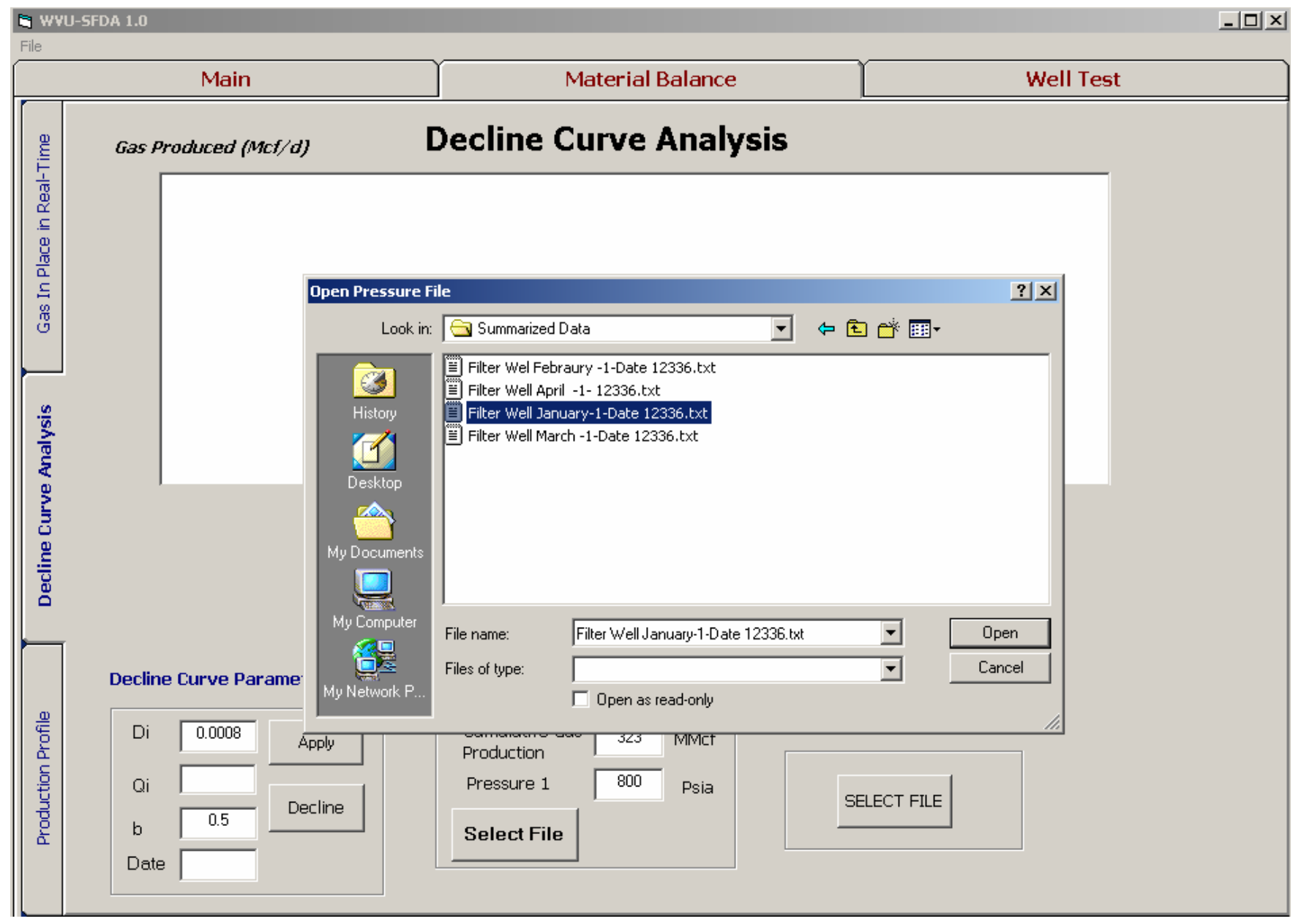


After selecting the first file, you will see the information about January in a very compressed way.

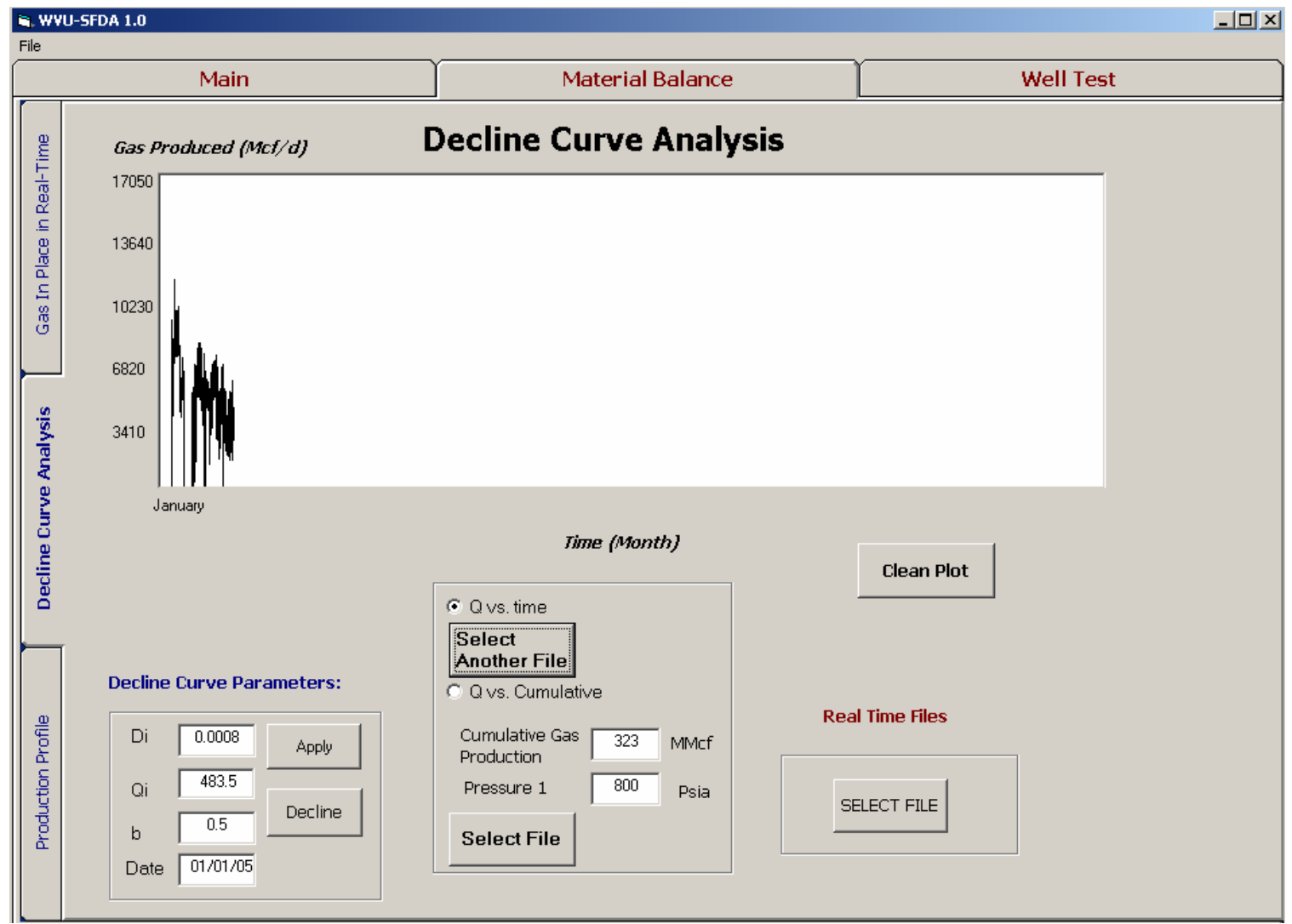

SFDA has automatically picked values for the initial flow rate (Qi) and Date of such flow rate:

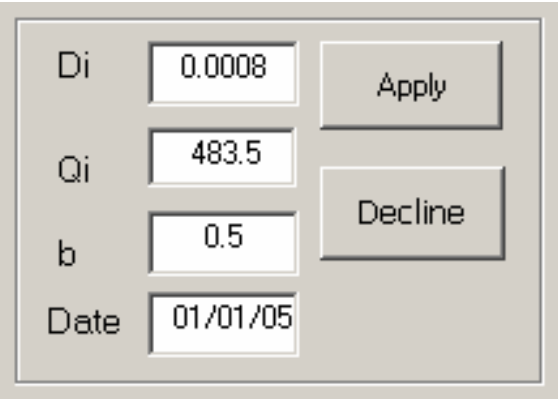


If you do not agree with a flow rate of $483.5 \mathrm{Mcf} / \mathrm{d}$; you can change it to a value you can estimate from the curve. For instance, $8500 \mathrm{Mcf} / \mathrm{d}$ :

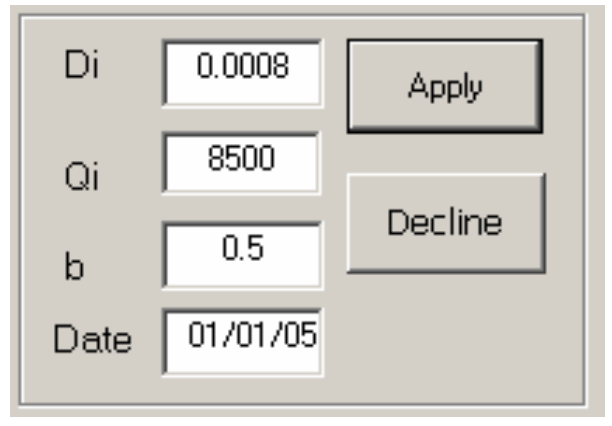

Now you will click "Apply" in order for the program to pick the date when a flow rate of 8500 is measured. After you click "Apply", you will again select the file containing the monthly summarized data you have just selected:

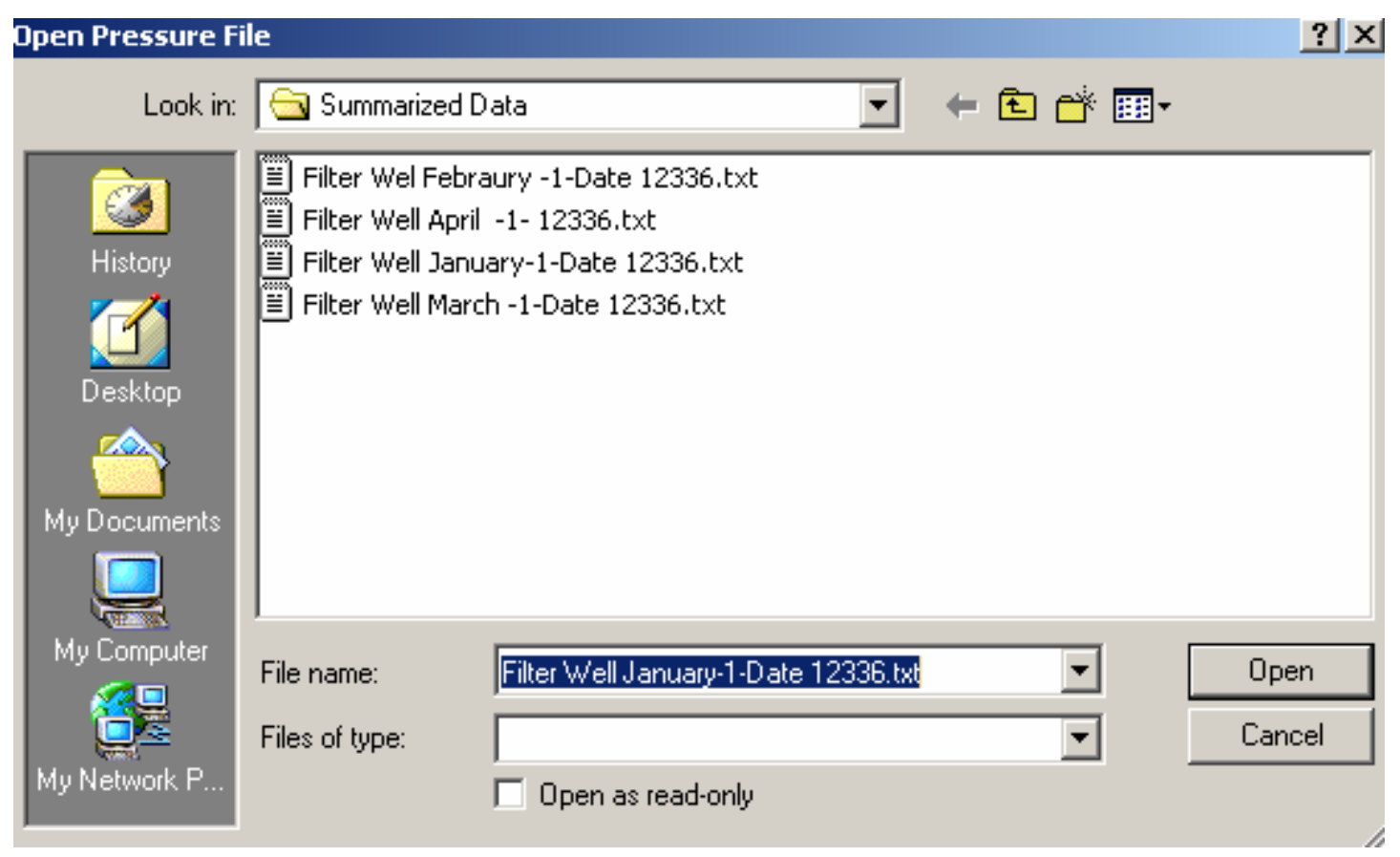


SFDA has now found the date when a flow rate of $8500 \mathrm{Mcf} / \mathrm{d}$ has taken place:

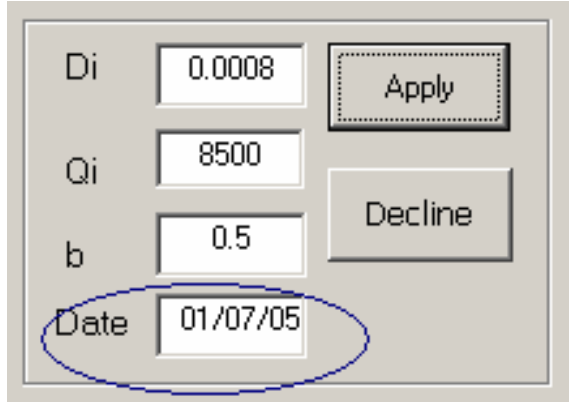

In addition, you can now select the next file. For this case would be February:

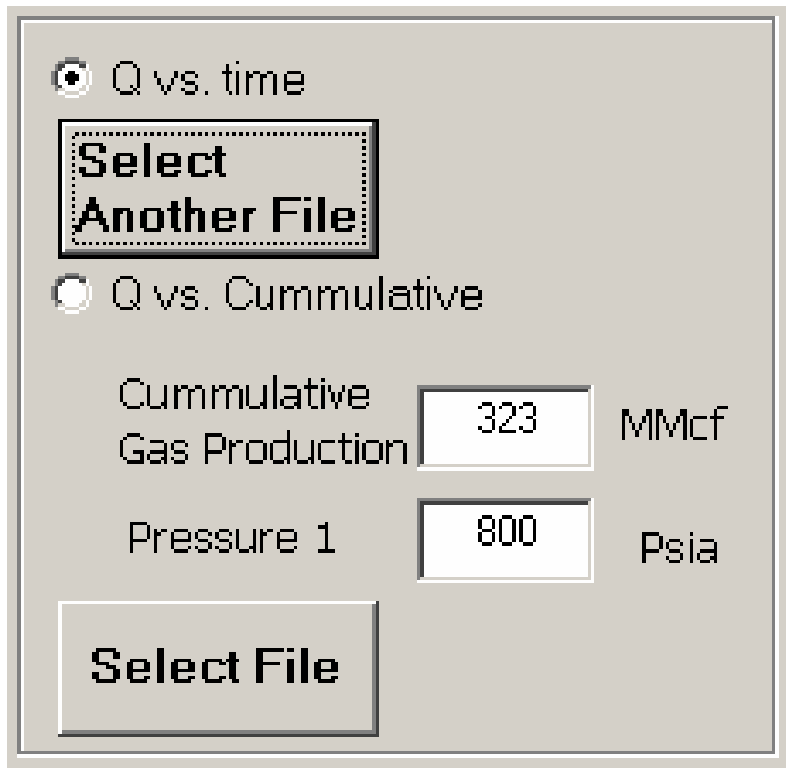


Select the file:

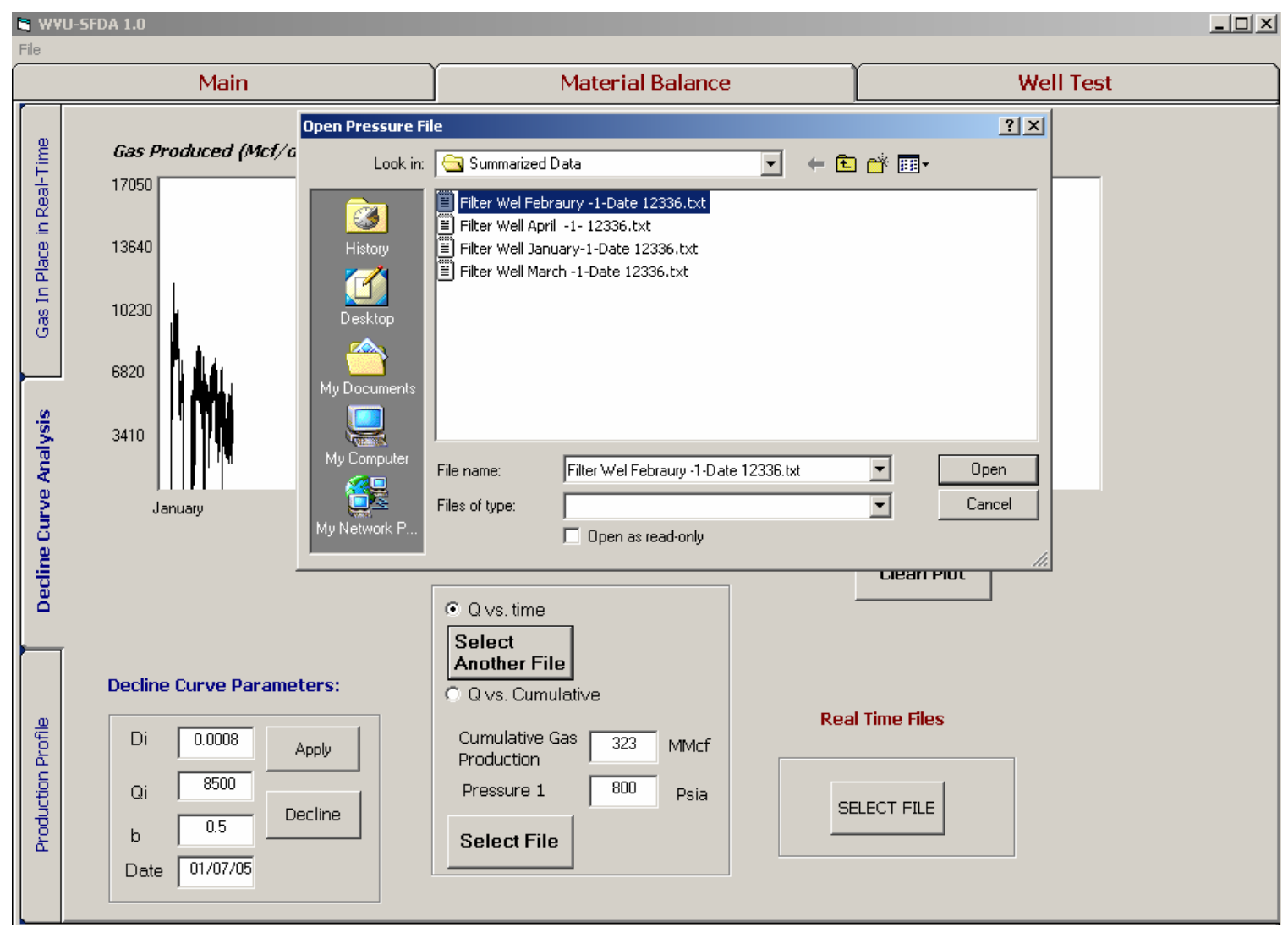


And the results would be:
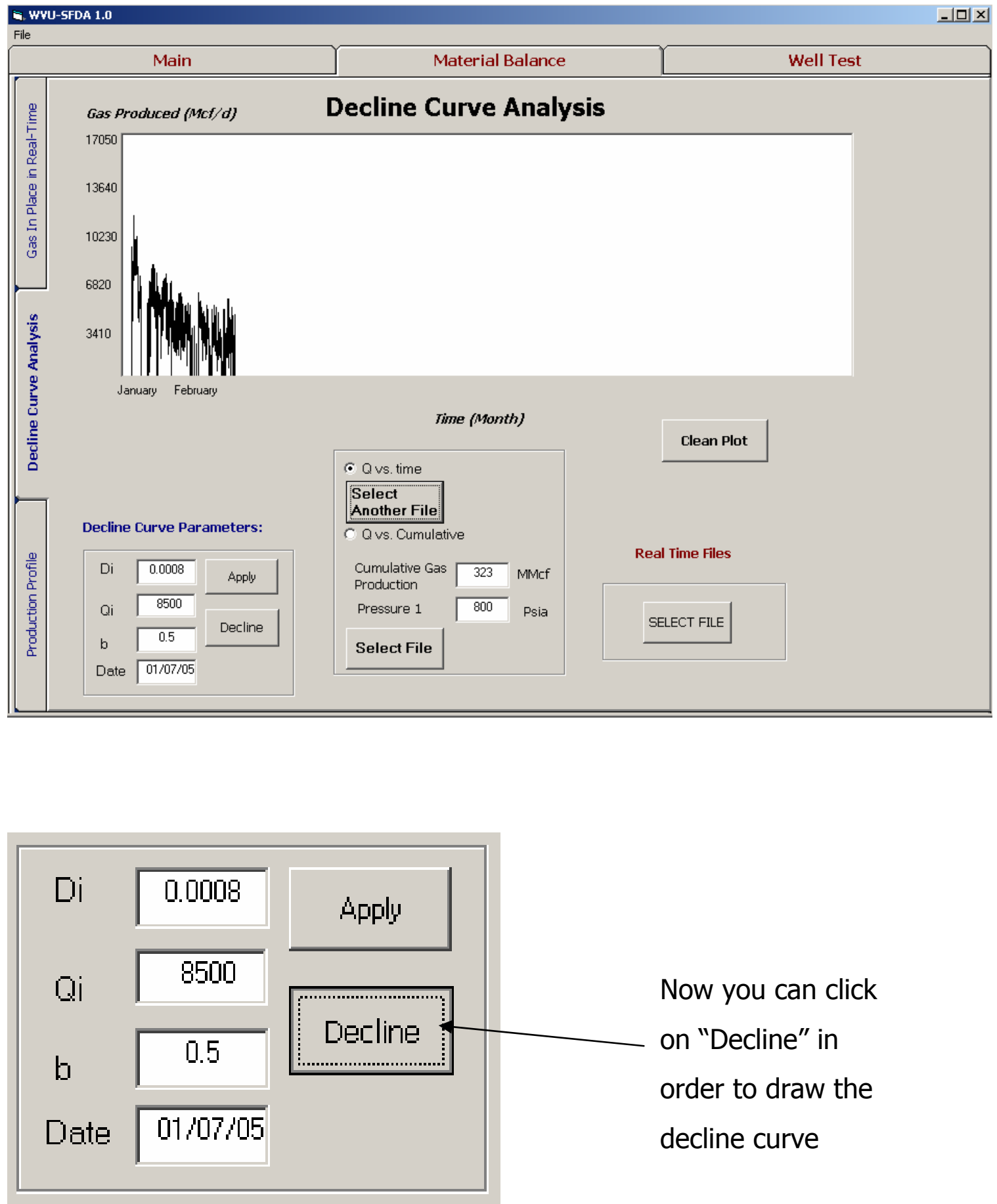


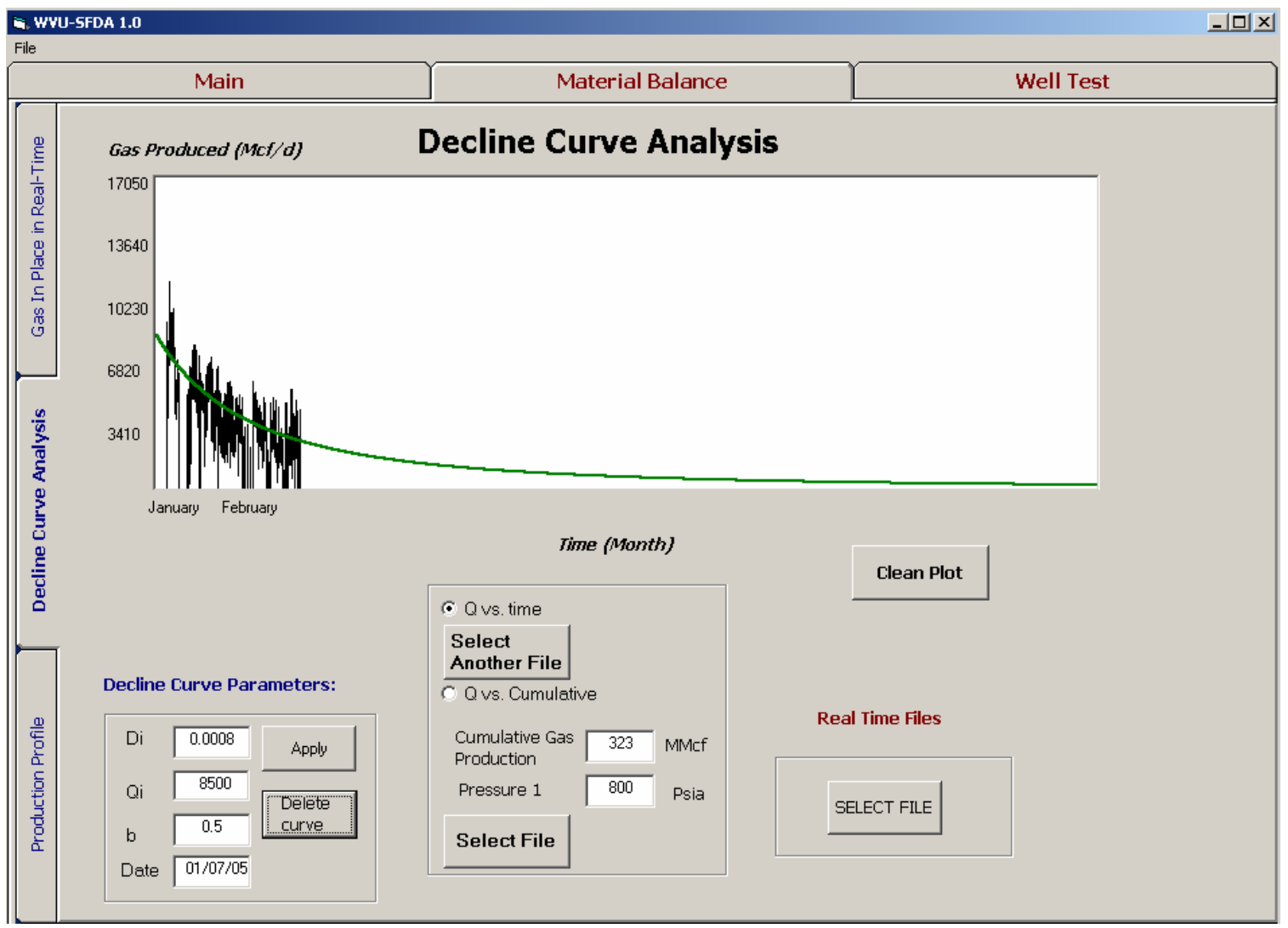

If you want to perform the decline curve analysis by plotting Gas production vs. cumulative gas instead of gas production vs. time you can simply select the option "Q vs. Cumulative" and click on "Select File" in order to select the first file which in this case would be January. You will follow the same procedure as before: After selecting the file, check on the Qi value, change it if you do not agree, click on "Apply", select again the file you have just selected, and click on "Decline" 


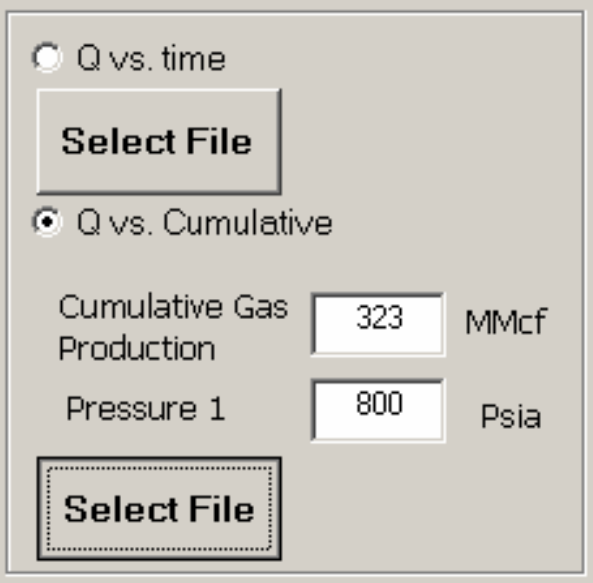

For this case you will see the following results:

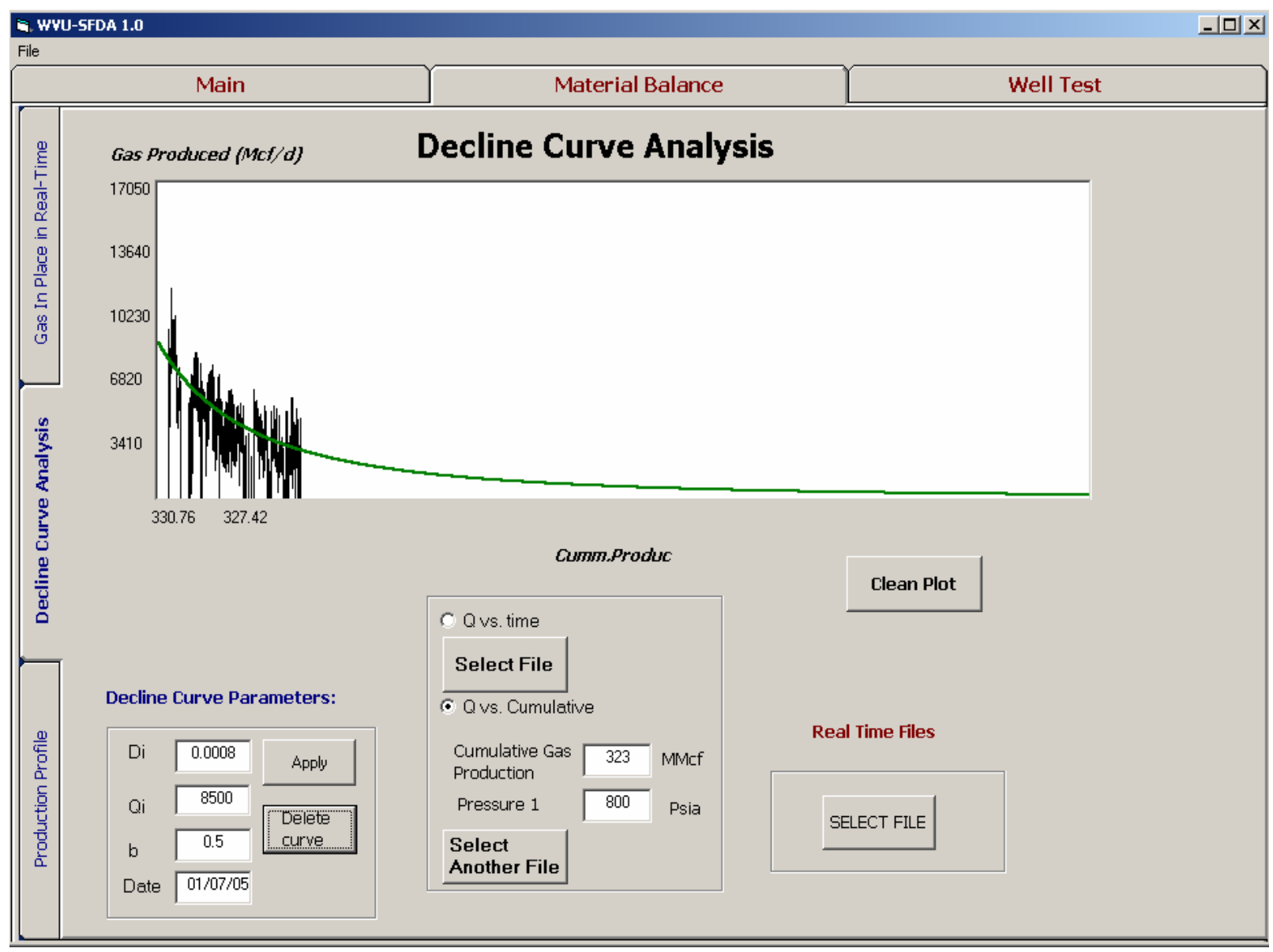


You can see how the real-time data lies on the decline curve. For instance, if you have used monthly data for January and February to draw the curve; you can upload daily real time from the following month (any day from March) in order to see whether such data follows the decline trend:

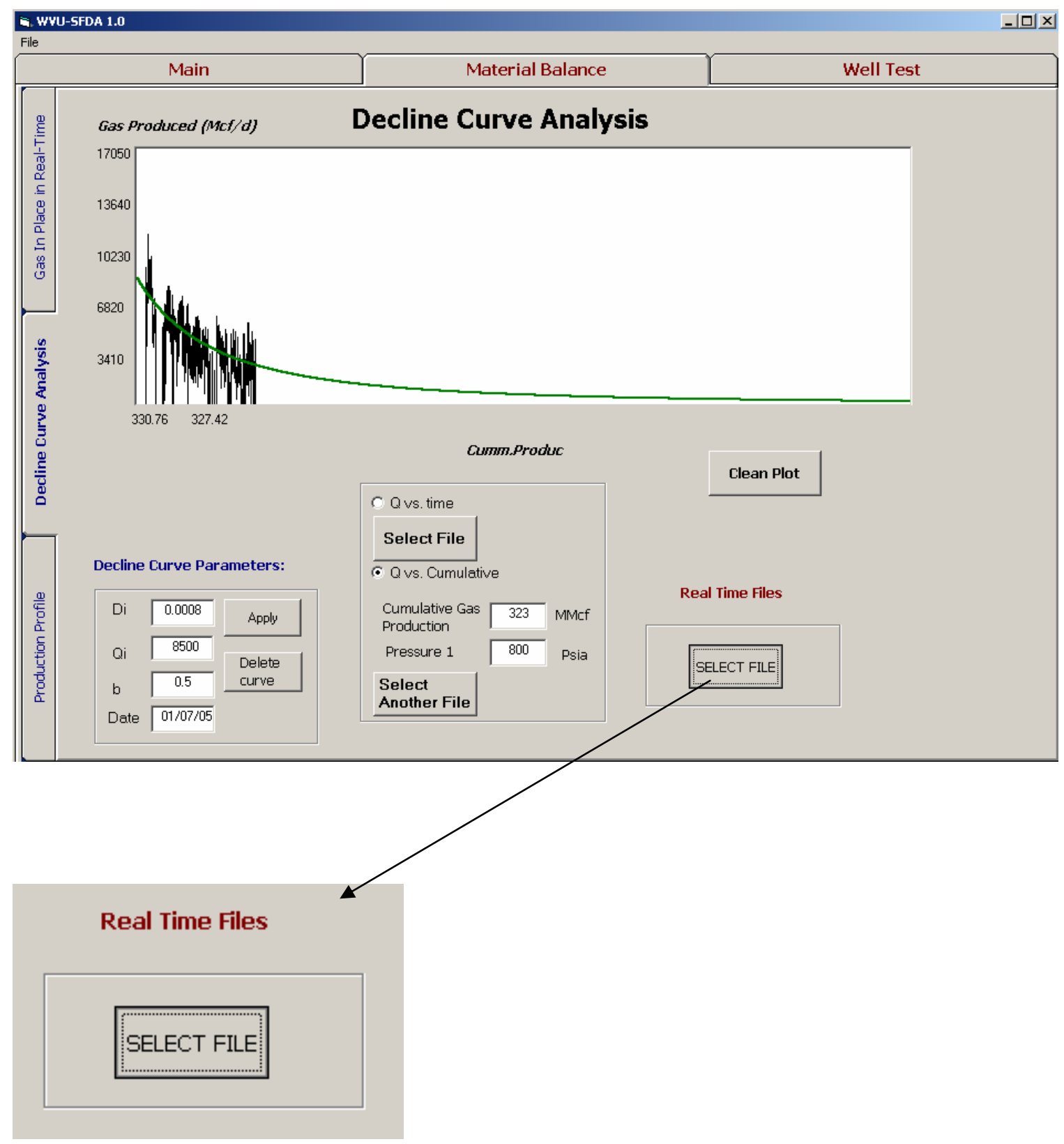


After clicking "SELECT FILE", look for the real time data from March:

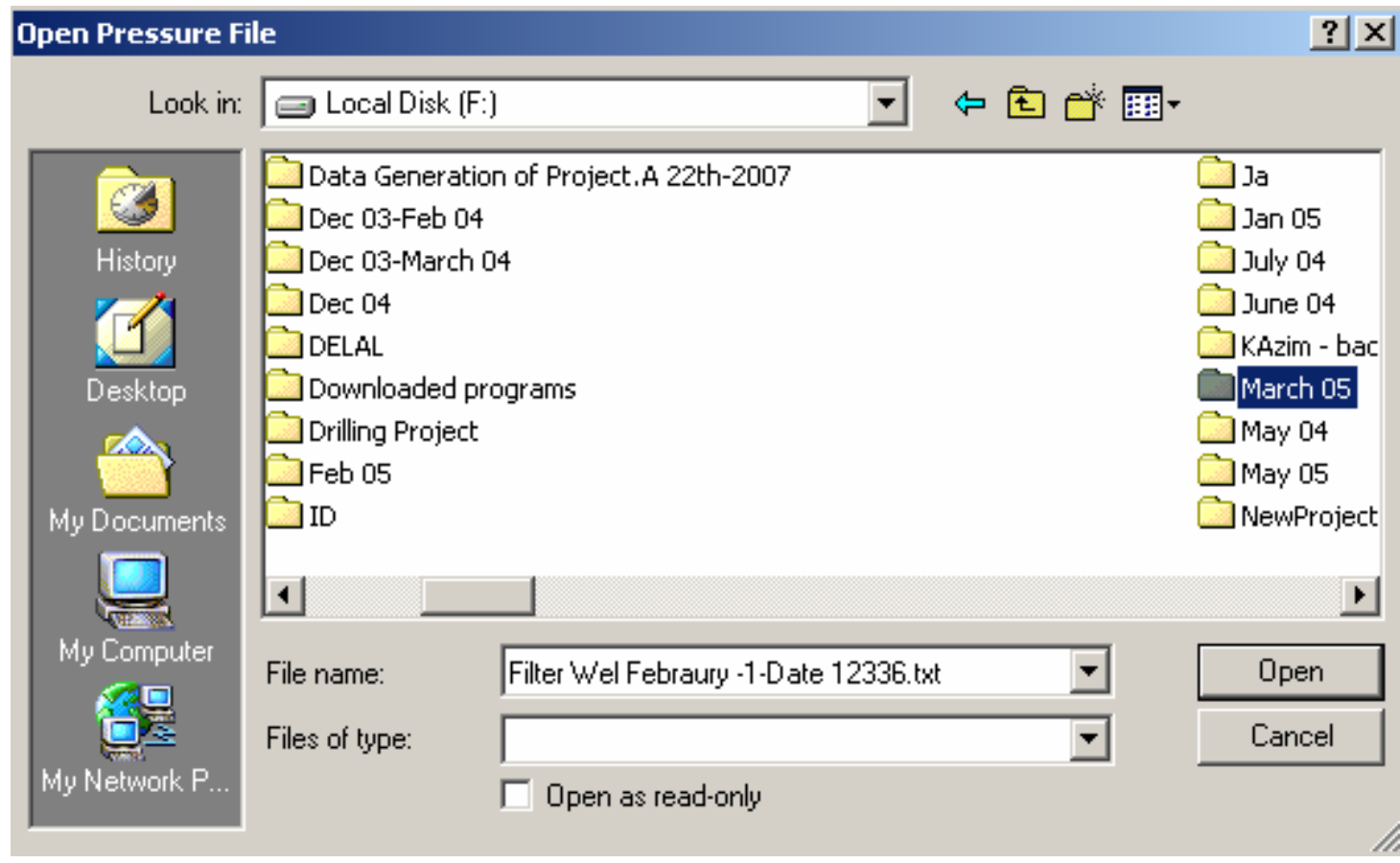

Select a file containing daily real-time data:

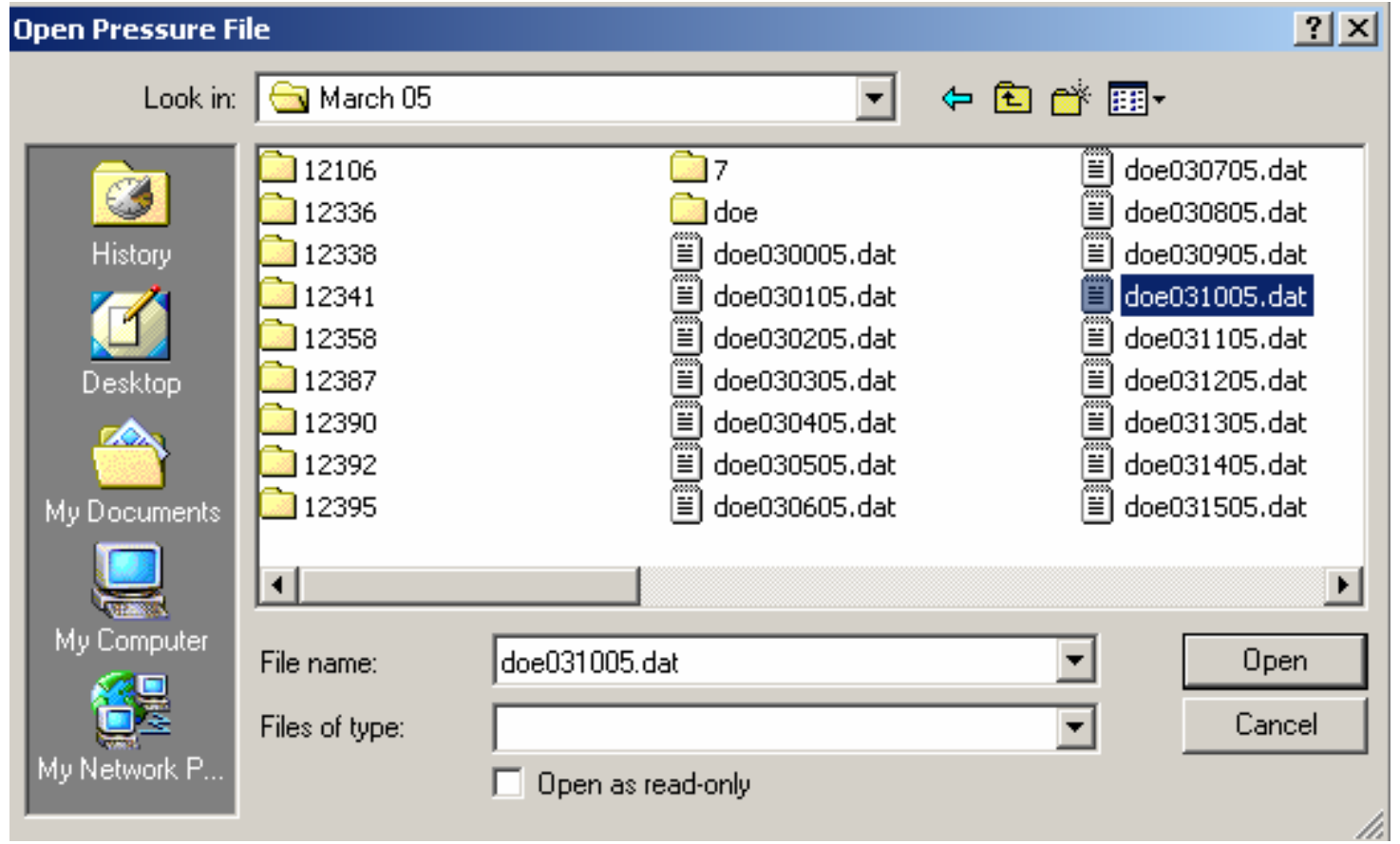


After double clicking on such file you will see:

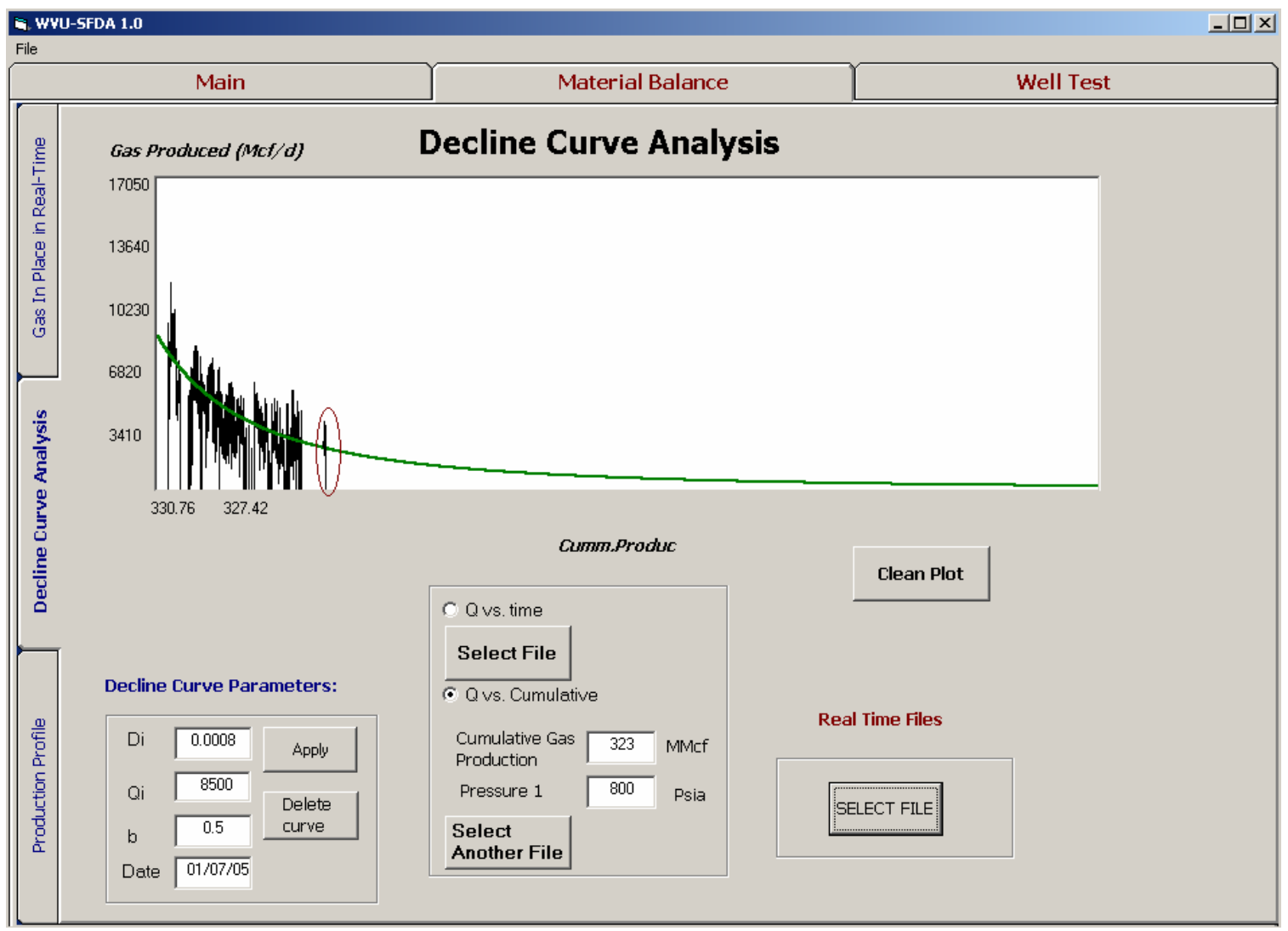

The data encircled in red correspond to a daily real-time file from March.

You can clean the plot by clicking on "Clean Plot" and the plot will be erased: 


\section{WELL TEST ANALYSIS}

SFDA has the capability of detecting pressure transient periods, saves all information in a folder and prepares the data for the user to analyze. The software will difference the transient from the presence of an outlier.

When the pressure increases to about $30 \%$ of the average of the last five values previously recorded, the program will start comparing the actual values with such average. If a suddenly increase in pressure value takes place, the software will read the next value and compare to the average of the last five values; if the next value is still very high, the program will repeat the process and will be kept going on about three more times until SFDA decides that a transient period has taken place. When this happens, the program will start saving pressure and time information in a folder; another window will show that a file is being created and opened for the process of the pressure transient analysis. Information getting into the system will be read in real time, send it to a file that keeps all information named "Raw Data", and save the information related to the transient identification to a file named "Well Testing". As soon as the pressure comes back down, the software will close such a file and the information will be ready for the user to analyze.

Conversely, if a very high increase in pressure takes place in which such a value is greater than the average of the last five points and then the program compares the next value with the average of the last five points resulting in a discontinuation of the pressure build up process; the program will detect immediately that an outlier is present in the data, it will remove it from the system and no information related to well testing analysis will be saved.

Once the computer program has detected a pressure transient period, you can upload the well test data by clicking on the file containing pressure transient data. First select the "Well Test" tab and the "Save Well Test" sub tab: 
File

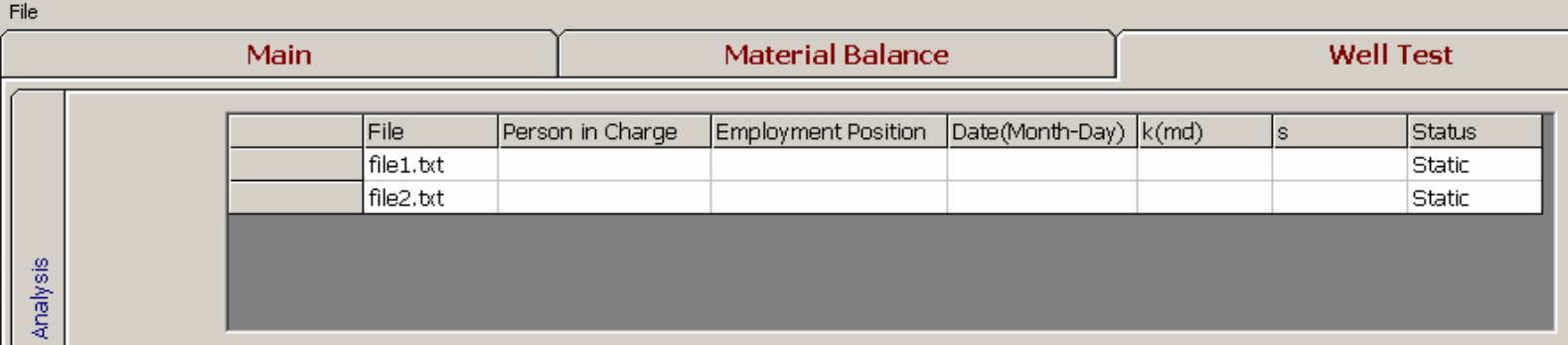

Pressure (psia)

Pressure vs. Time

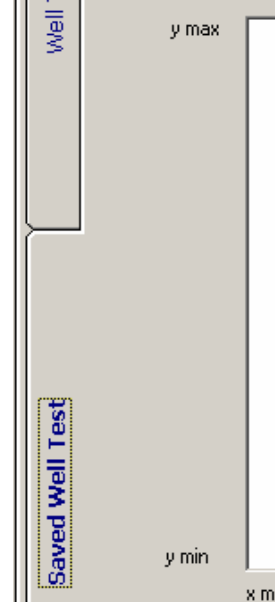

$x \min$

\begin{tabular}{|c|c|c|c|c|c|}
\hline File & Person in charge & Employment Pasition & Date(Month-CDay') & $k(m d)$ & Status \\
\hline file1,tyt & & & & & Staticic \\
\hline filez, tyt & & & & & Staticic \\
\hline
\end{tabular}

\section{Click on here}



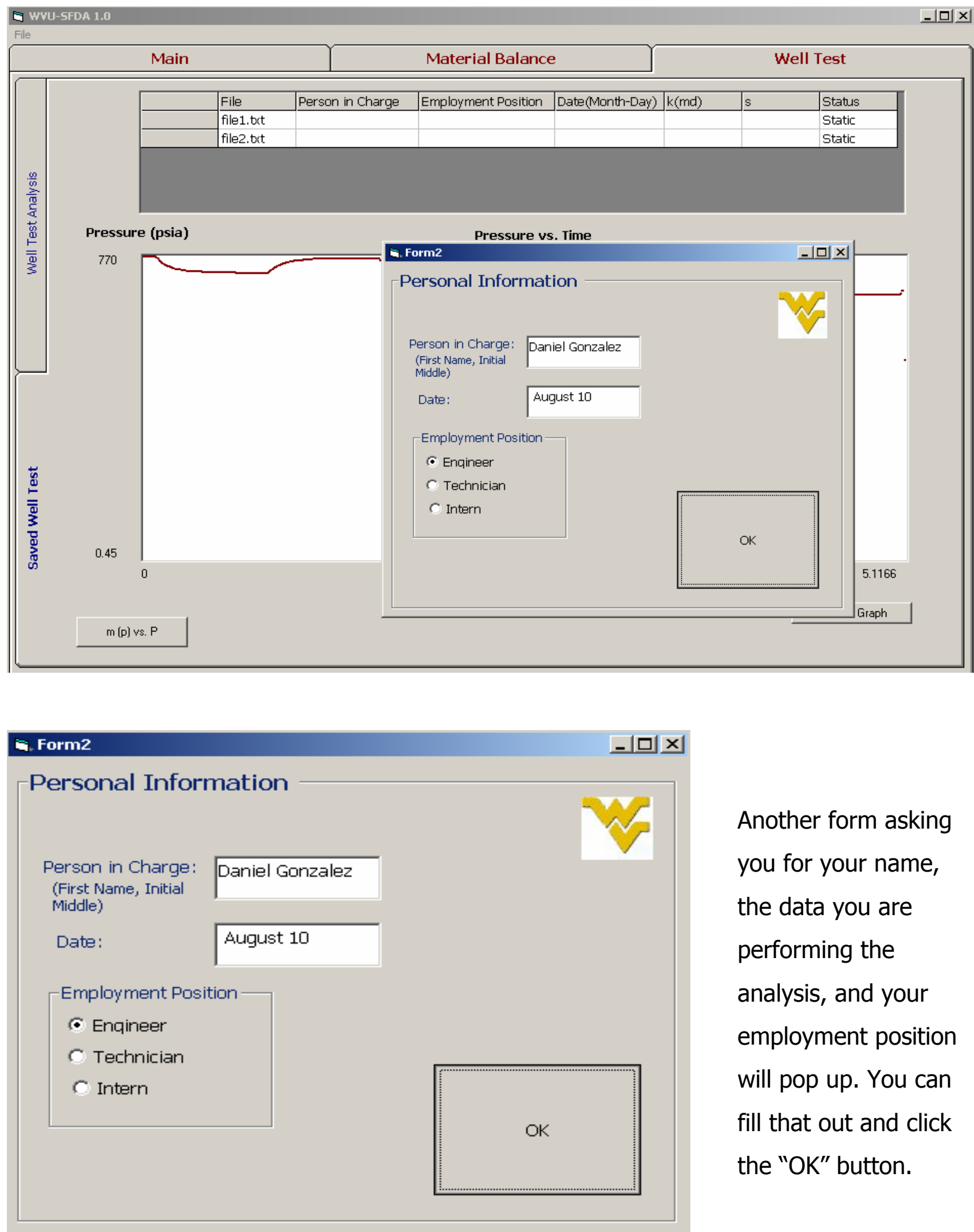

Another form asking

you for your name, the data you are performing the analysis, and your employment position will pop up. You can fill that out and click the "OK" button. 
After clicking the "OK" button, you will be able of selecting an area. Then you can click on the Well Test Analysis sub tab where you will see the Horner plot:

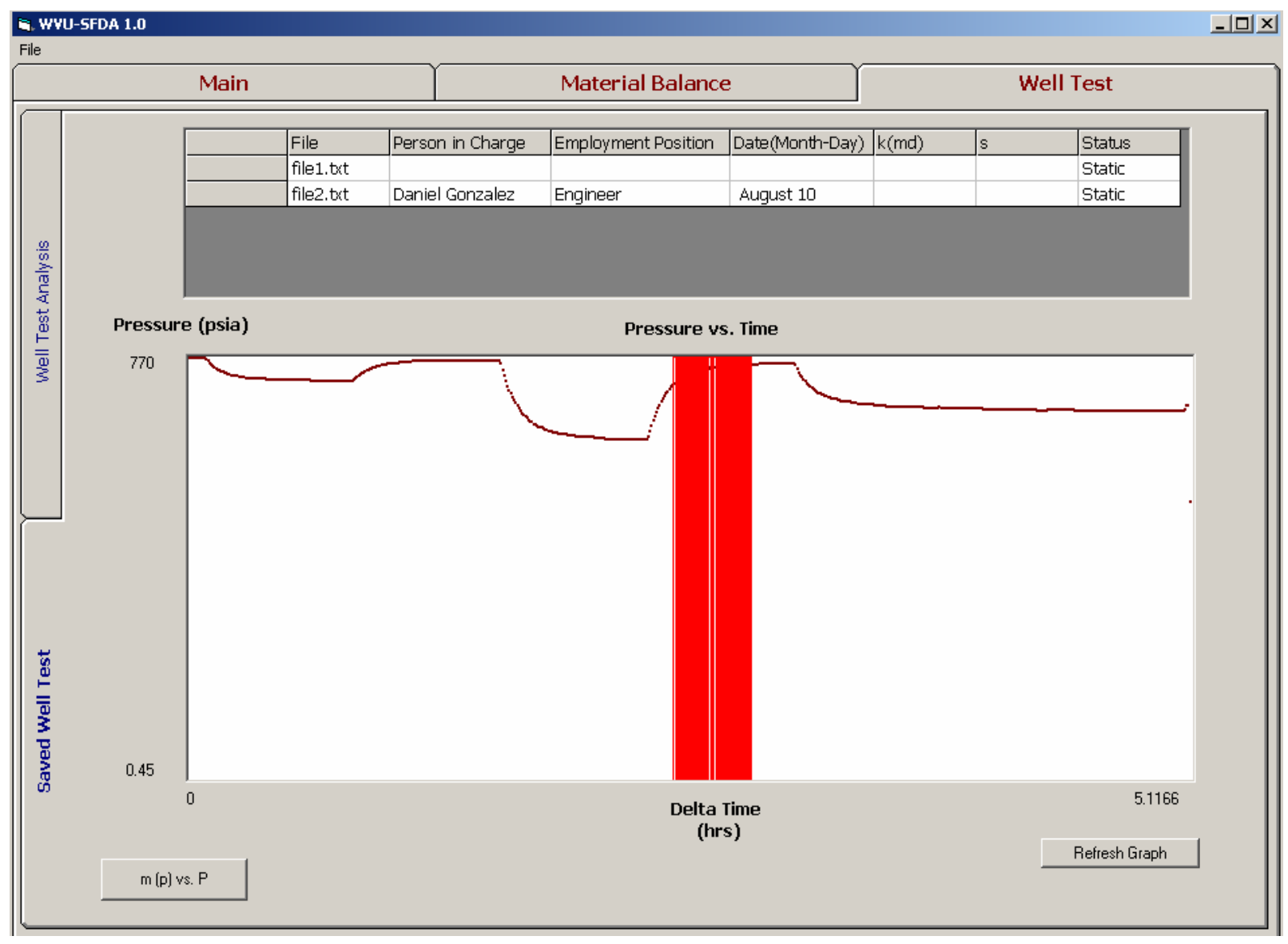


You will see the Horner Plot of the selected area:

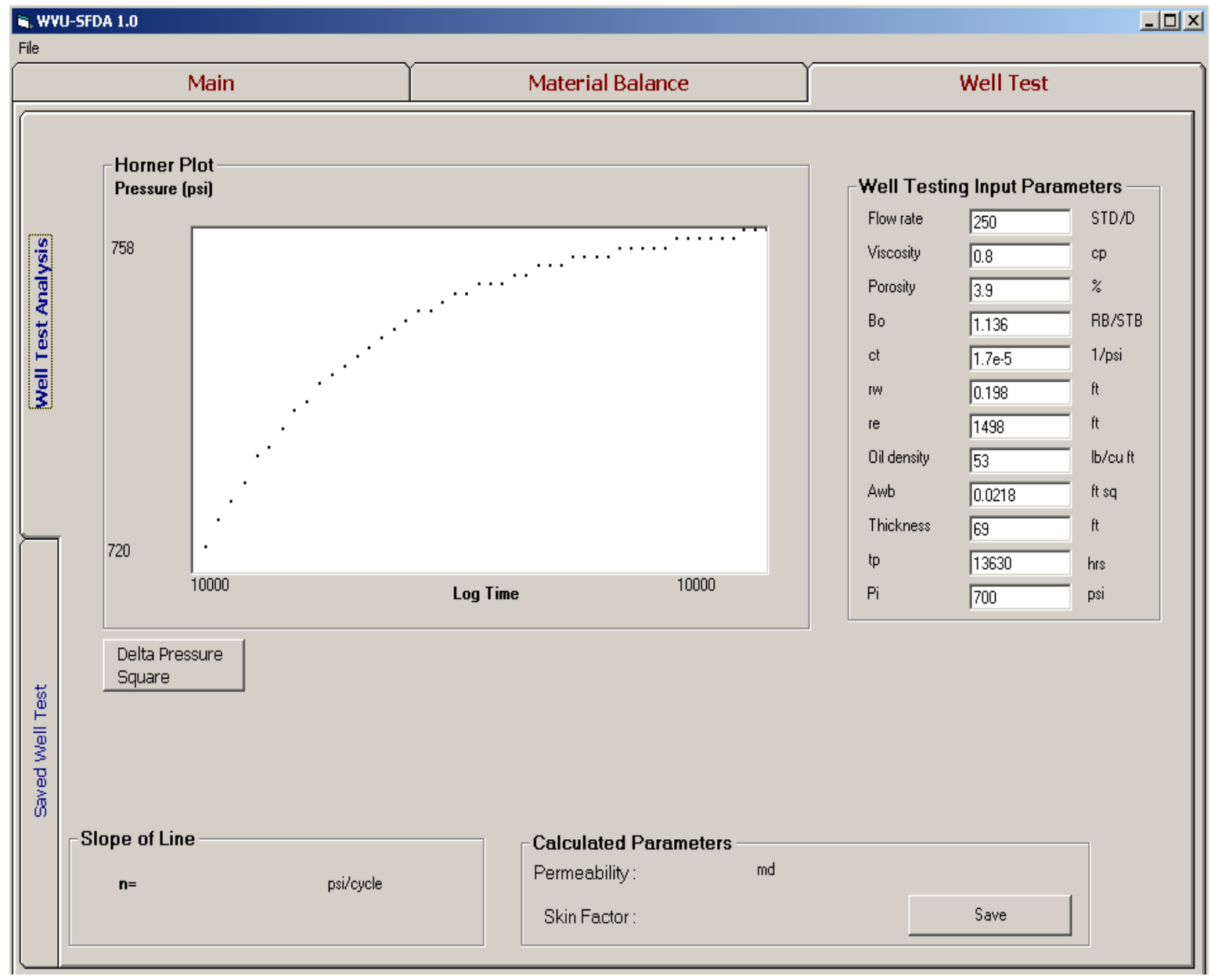


You can now click on the picture and draw line that will permit you obtain value for permeability and skin factor according to the conditions shown in the table on the right side:

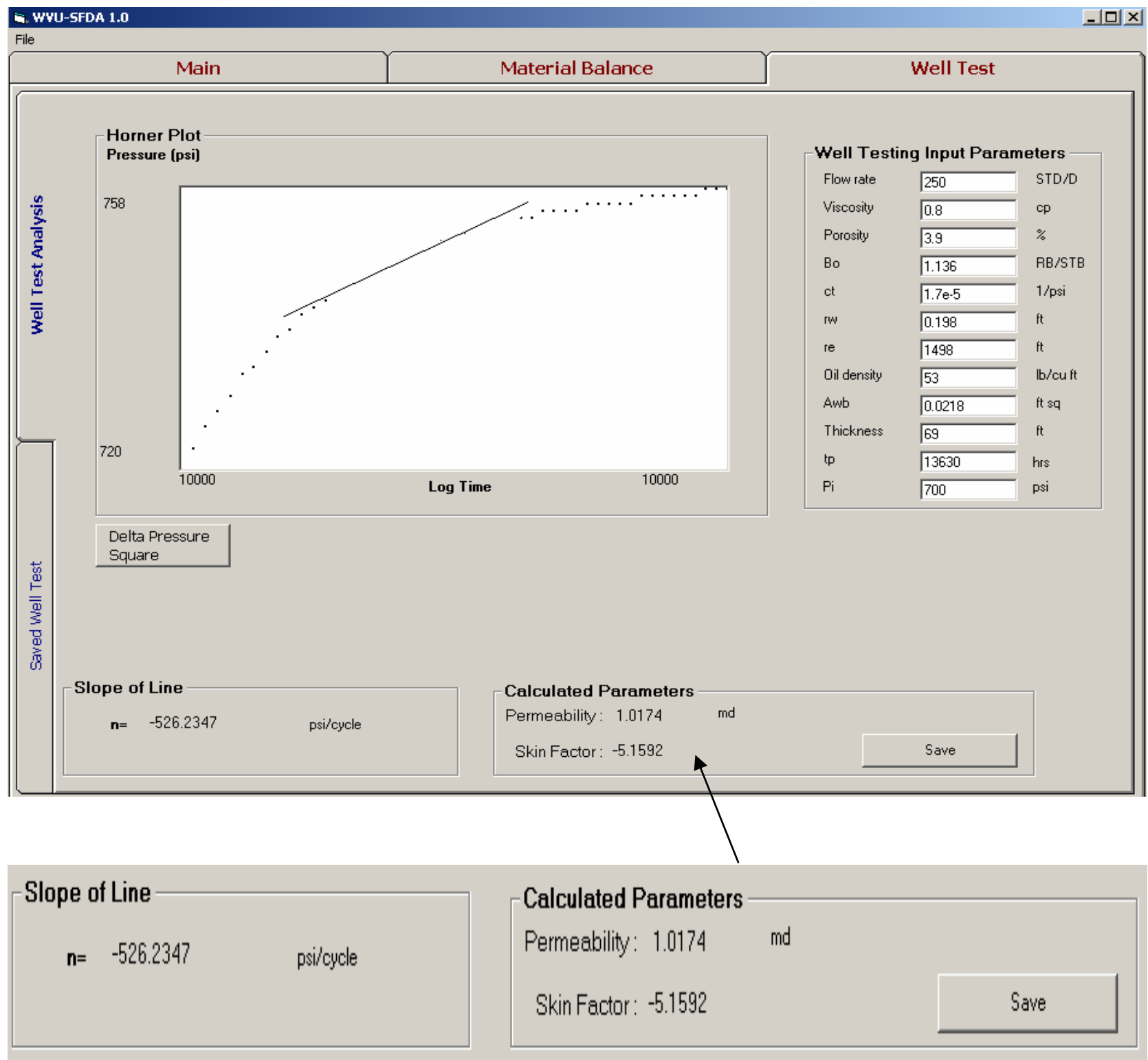




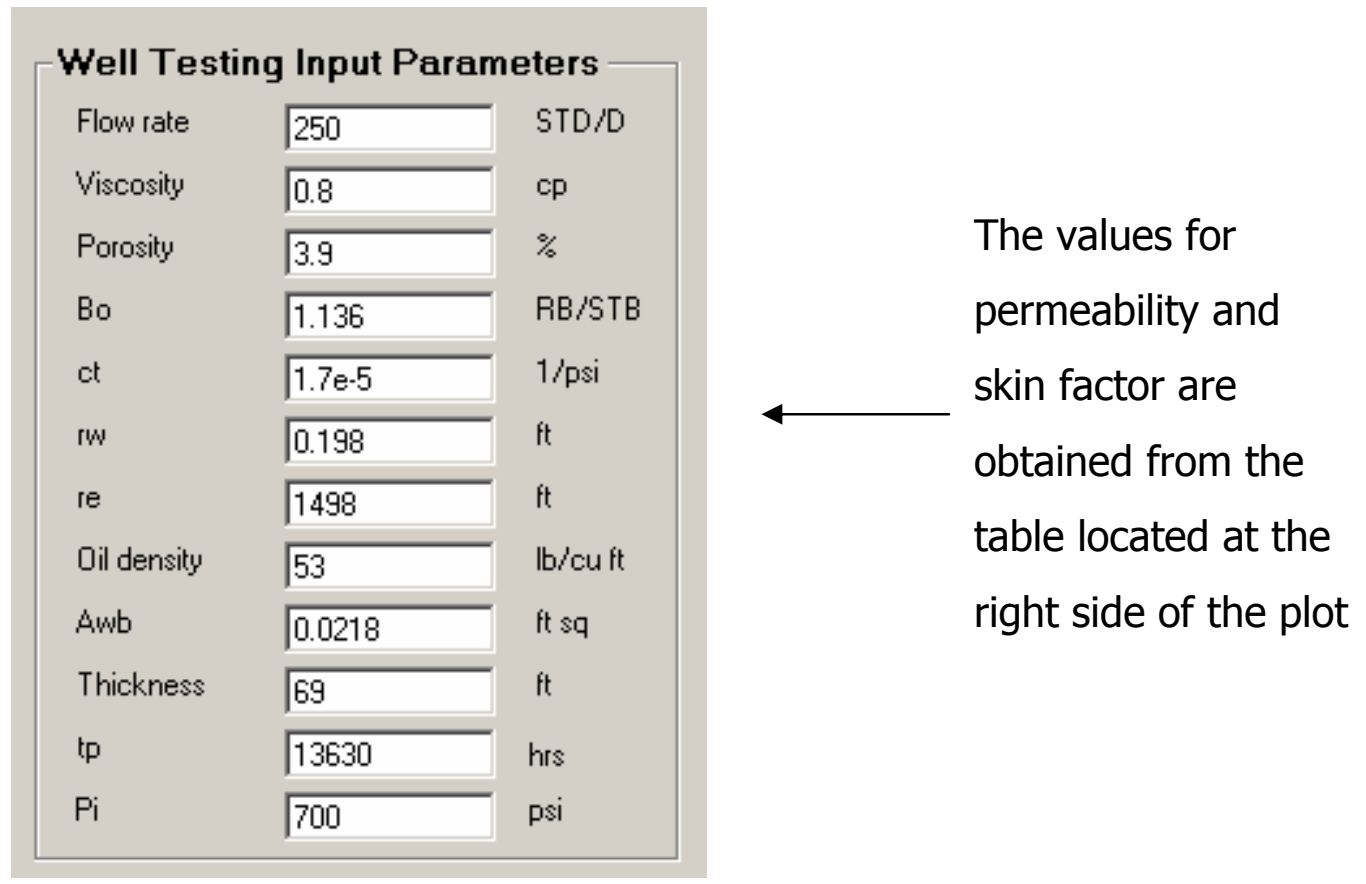

Well Test Analysis using Pseudo Pressures vs. Pressure:

Whenever a pressure transient period has been detected by the program, the user will have the option of plotting pseudo pressure versus pressure and select an area to draw the Horner plot.

You can click on the "Save Well Test" tab again and then click on the button located at the bottom left part of the plot to obtain a plot of pseudo pressure vs. pressure

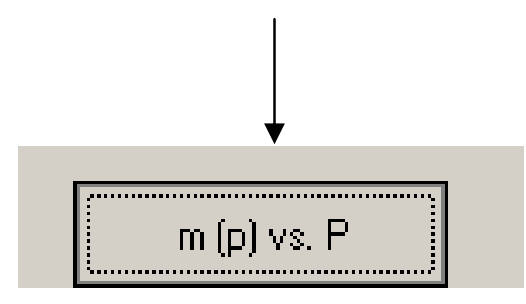


You will see the following:

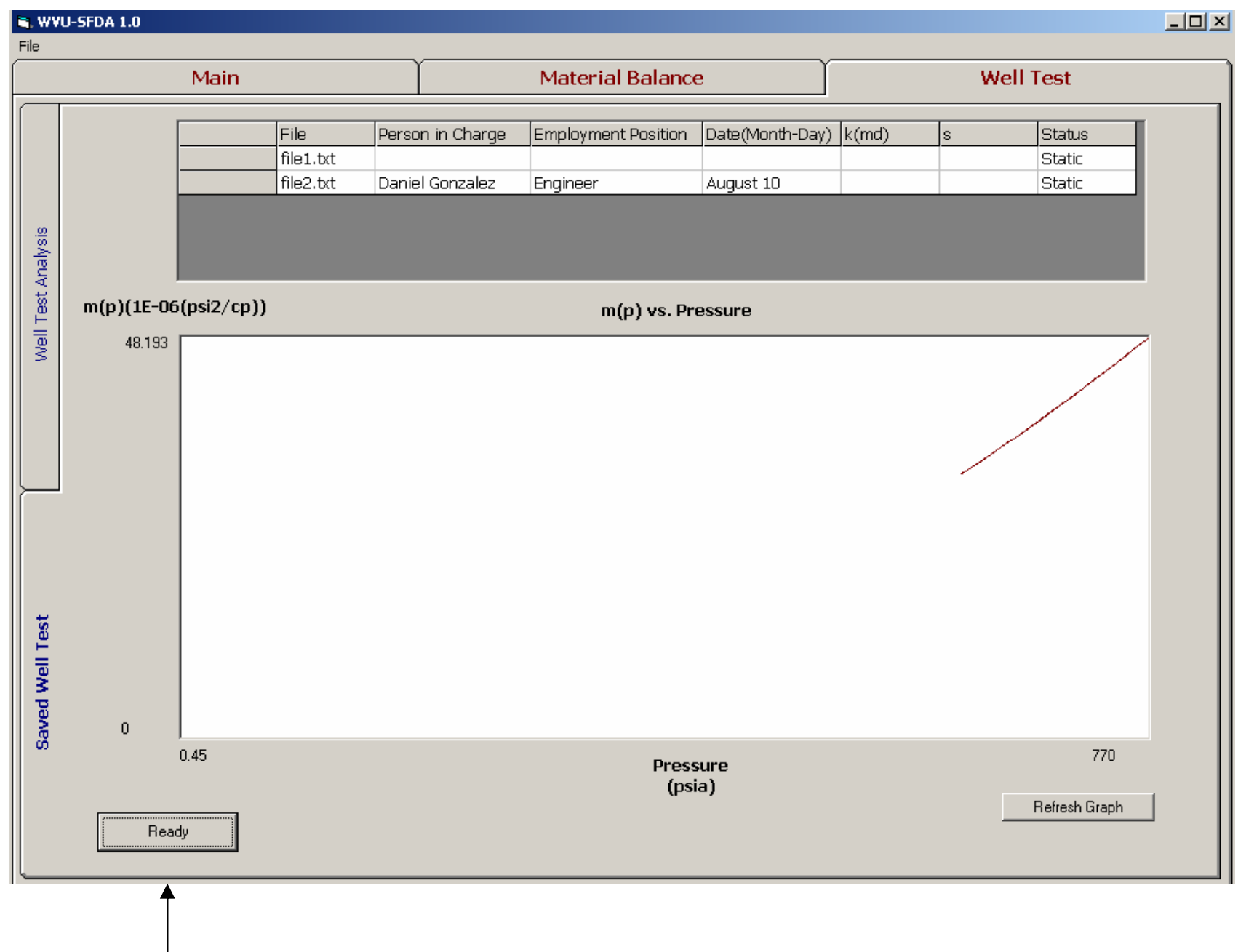

The "m (p) vs. P" button caption will change to "Ready"

$$
m \text { (p) vs. } P
$$

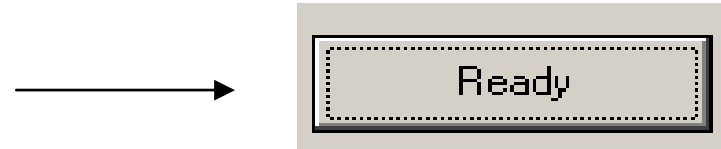


You can select an area and switch again to the "Well Test Analysis" tab in order to carry out the Horner plot for the area just selected:

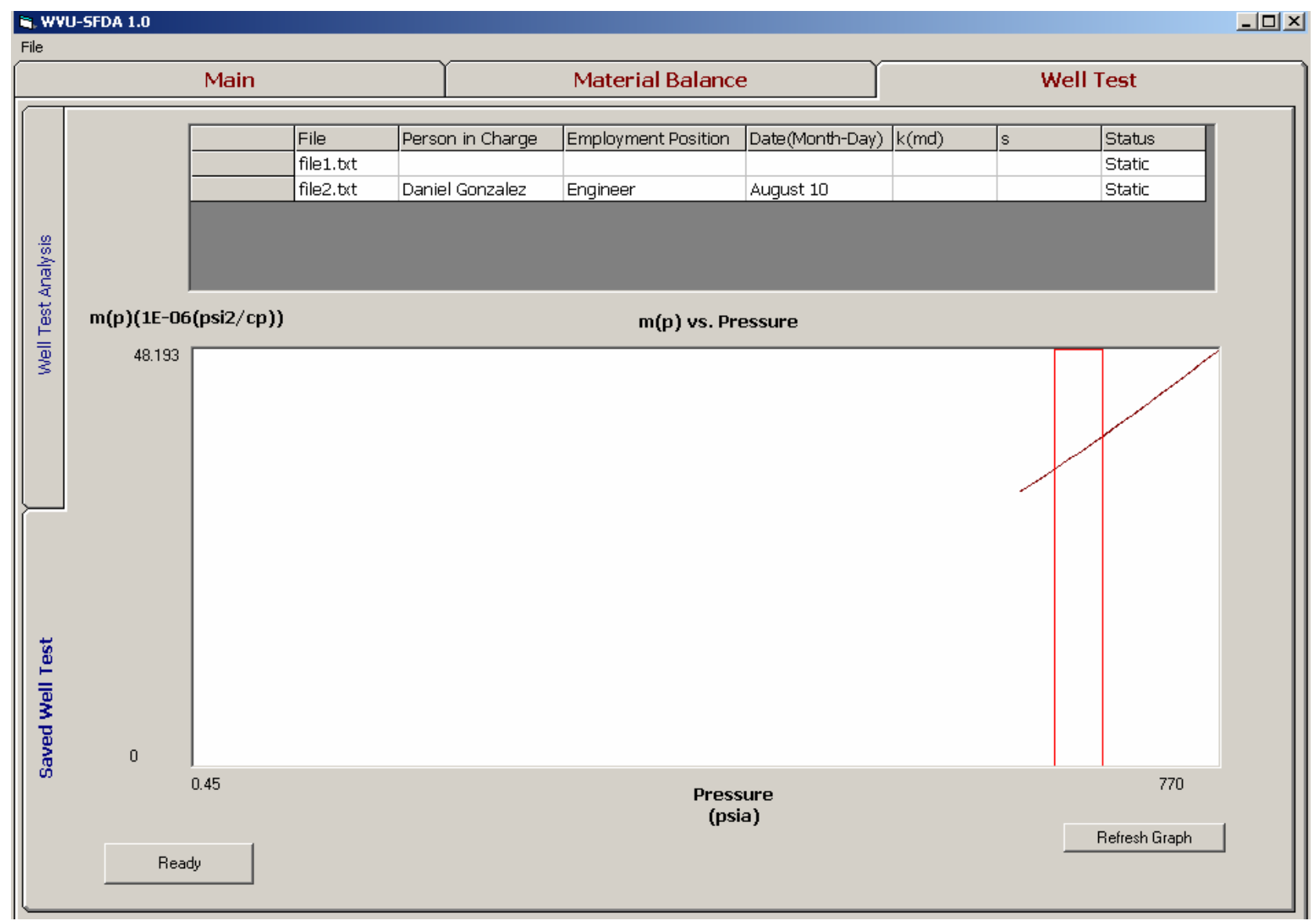


You will now have a Horner plot of pseudo pressure vs. log of time, after drawing a line you can get value for permeability and skin factor:
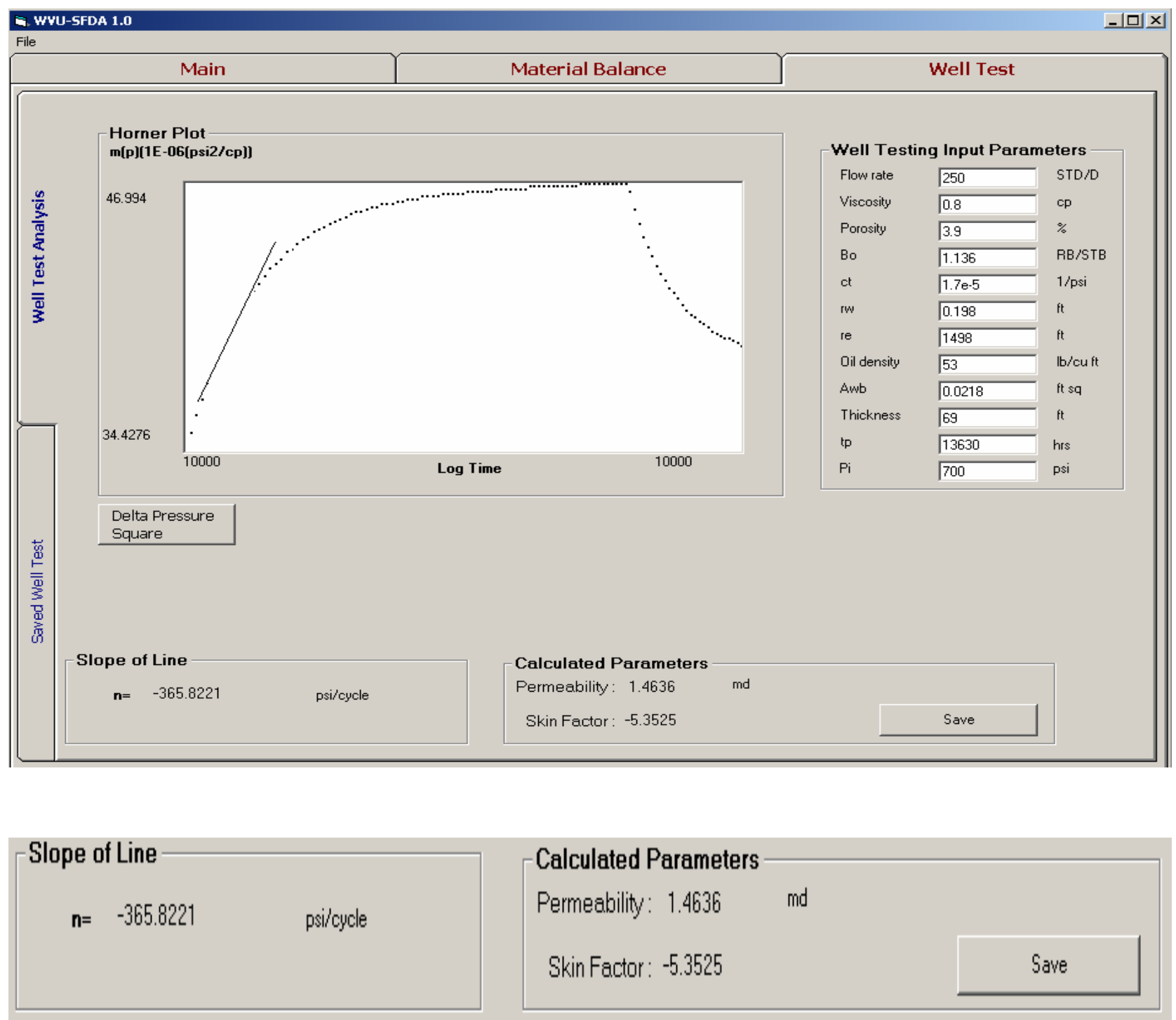

If you want to save such information click on the "Save" button and the information will be saved 
You can see the saved values for permeability and skin factor in the grid:

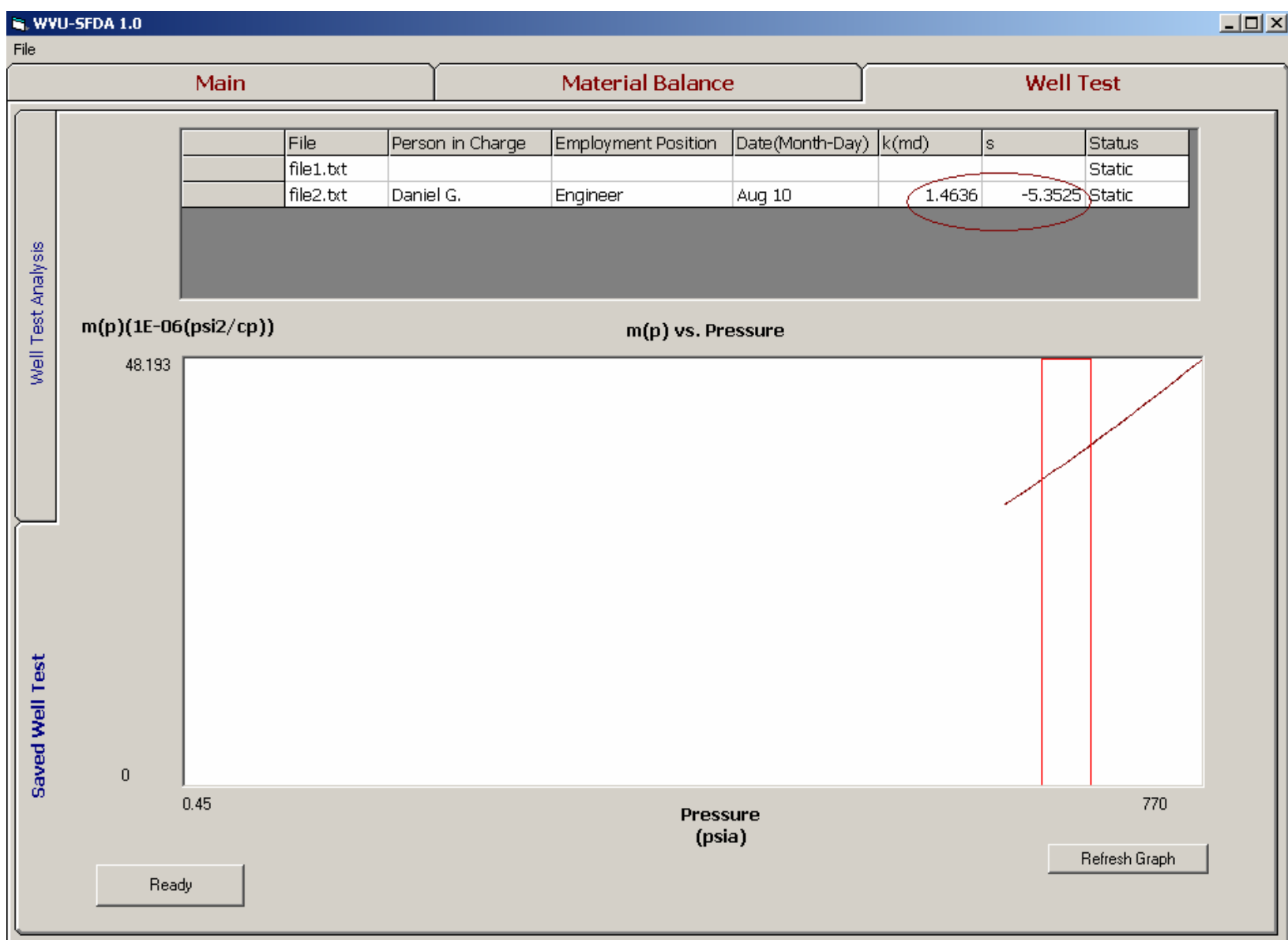

\begin{tabular}{|l|l|l|l|l|l|l|l|}
\hline & File & Person in Charge & Employment Position & Date(Month-Day) & k(md) & s & Status \\
\hline & file1.txt & & & & & & \\
\hline & file2.txt & Daniel G. & Engineer & Aug 10 & 1.4636 & -5.3525 & Static \\
\hline
\end{tabular}


You can always clean the plot by clicking on "Refresh Graph"

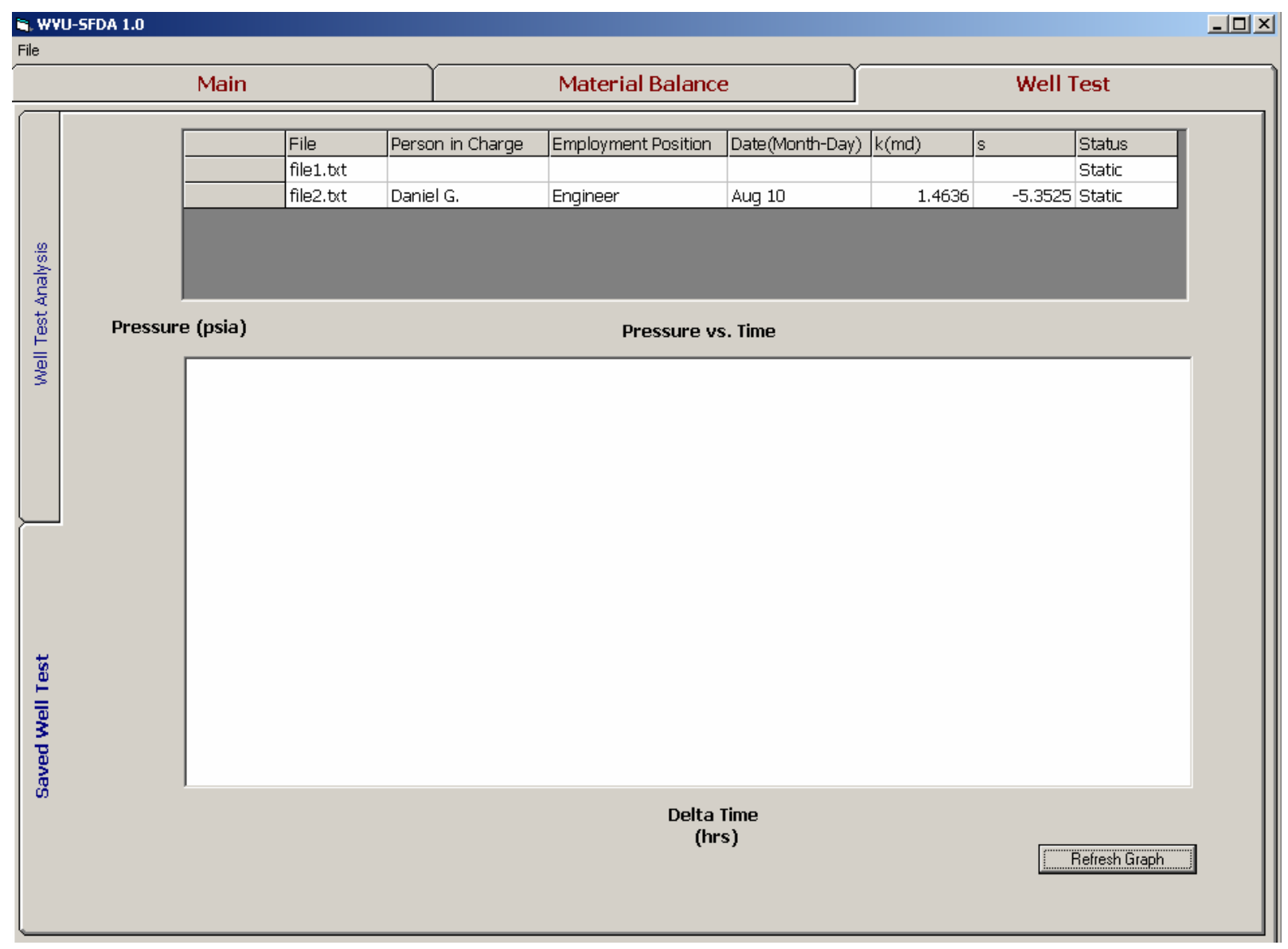


Well Test Analysis using Delta Pressure Square:

You can also select an area from a drawdown period and perform Semi-Log plot using the delta pressure square vs. log of time. Then you can draw the slope to obtain permeability and skin factor from drawdown periods:

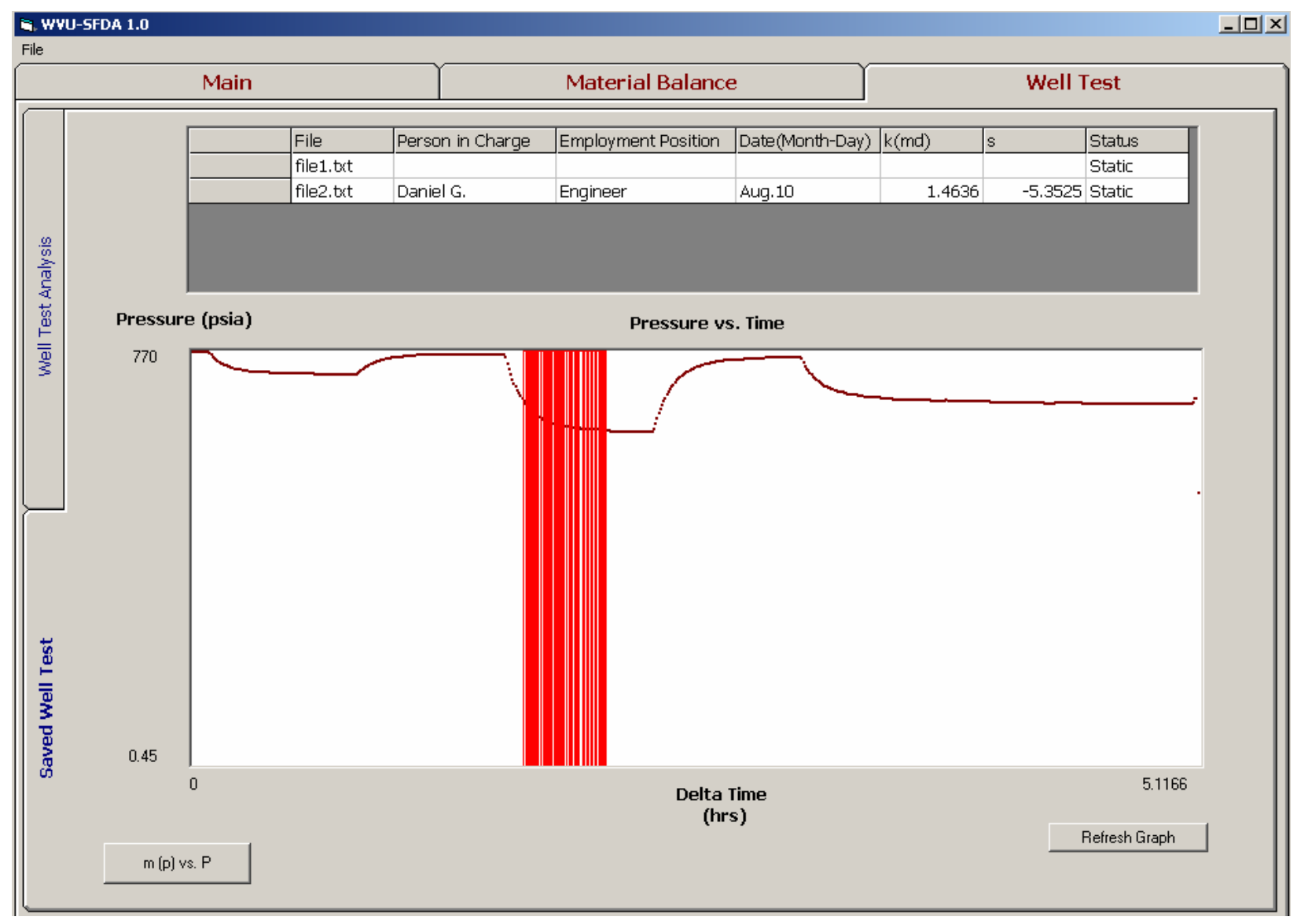


You will see the following plot. Now you can click on the "Delta Pressure Square" button in order to convert the pressure to delta pressure squares:

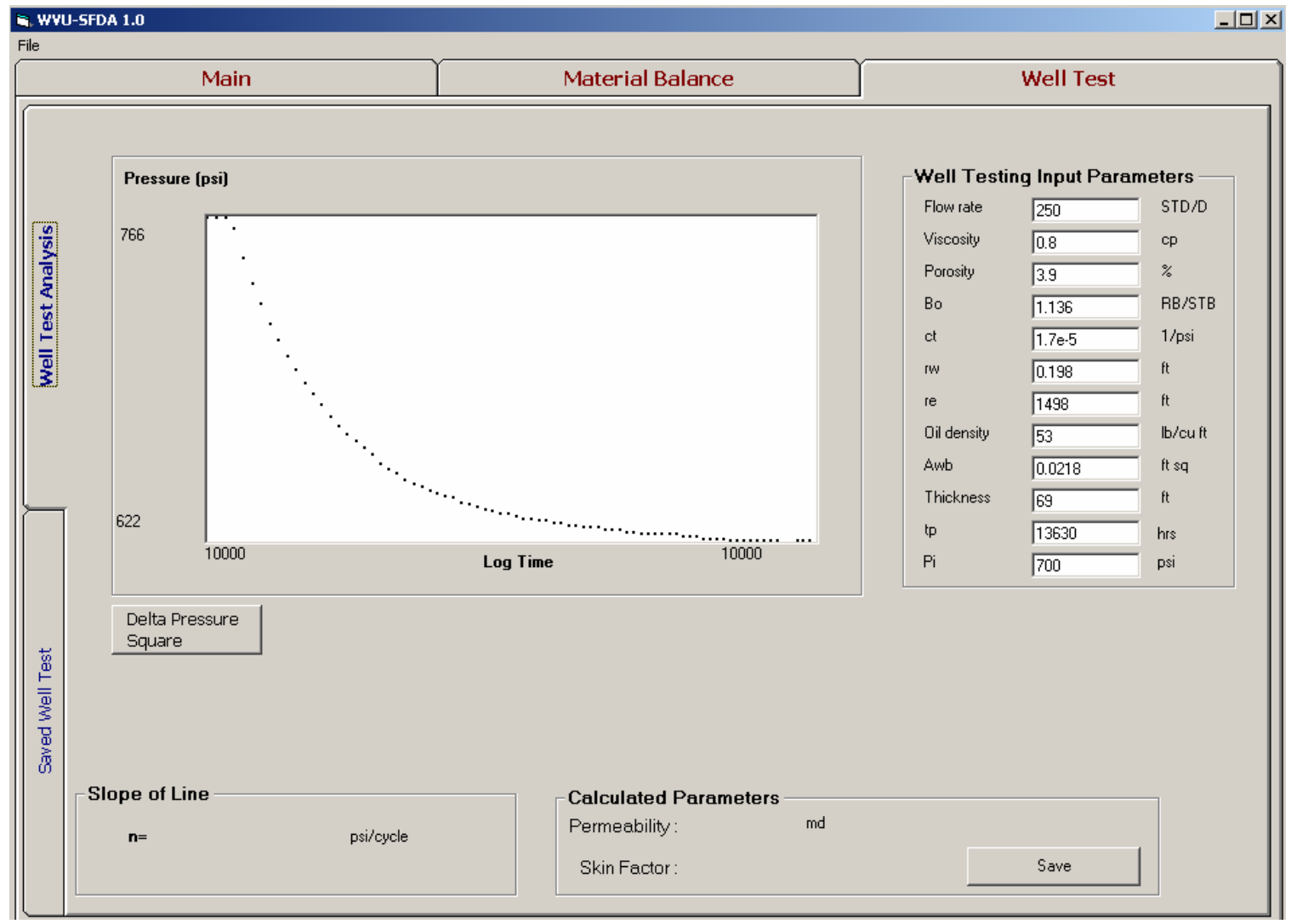


After clicking on the "Delta Pressure Square" button you will see a drawdown period of Delta Pressure Squares vs. log of time and again you can draw a line and obtain values for permeability and skin factor:

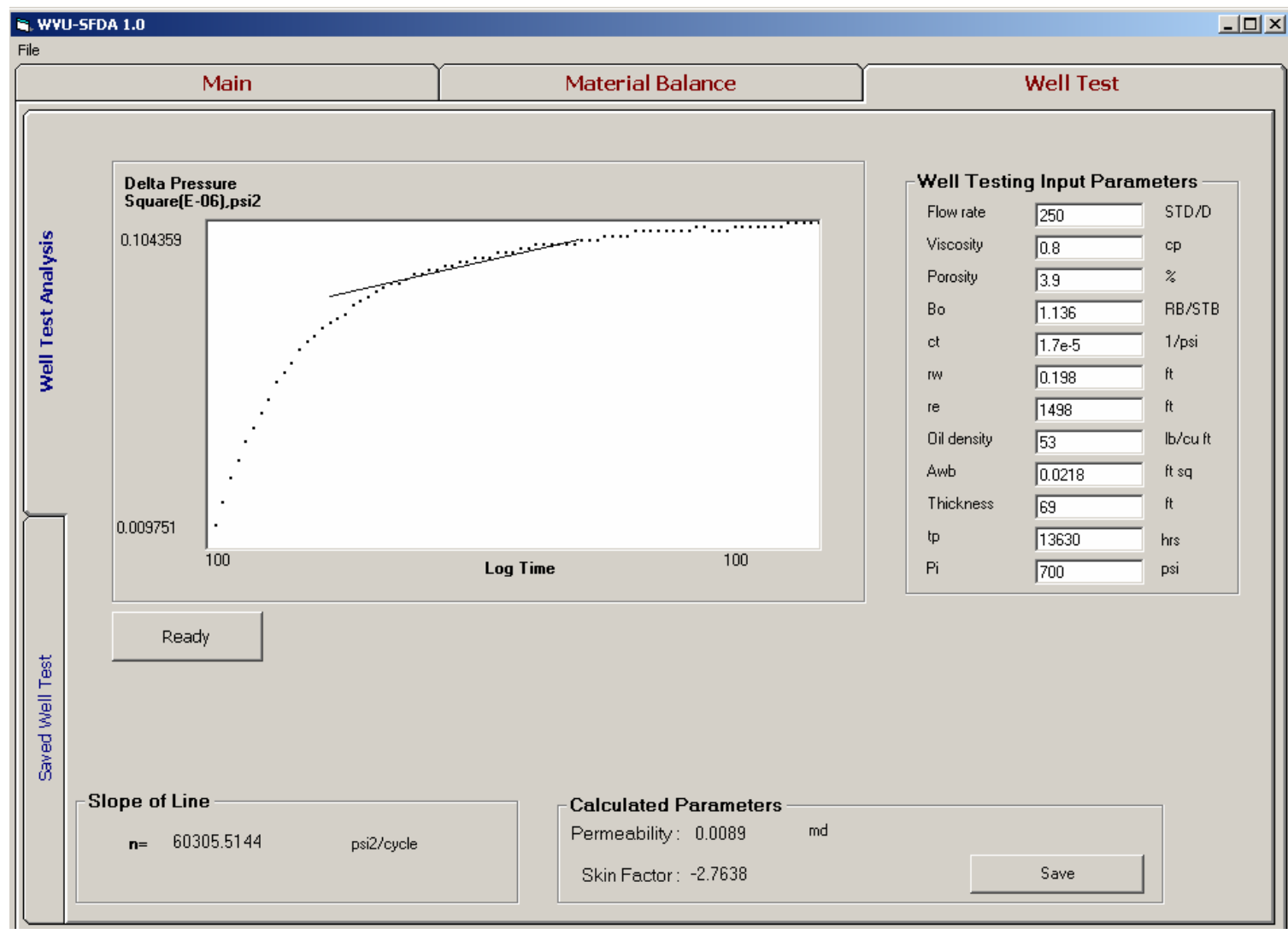

